\title{
Mathematik
}

\section{The Universal Functorial Equivariant Lefschetz Invariant}

\author{
Inaugural-Dissertation \\ zur Erlangung des Doktorgrades \\ der Naturwissenschaften im Fachbereich \\ Mathematik und Informatik \\ der Mathematisch-Naturwissenschaftlichen Fakultät \\ der Westfälischen Wilhelms-Universität Münster
}

\author{
vorgelegt von \\ Julia Weber \\ aus Berlin
}

$-2005-$ 
Dekan:

Erster Gutachter:

Zweiter Gutachter:

Tag der mündlichen Prüfung.

Tag der Promotion:
Prof. Dr. Klaus Hinrichs

Prof. Dr. Wolfgang Lück

PD Dr. Michael Joachim

30. Mai 2005

13. Juli 2005 


\section{Contents}

1 Motivation and Basic Concepts 1

1.1 The Lefschetz Number . . . . . . . . . . . . . . . . . . . 1

1.2 Modules over Categories . . . . . . . . . . . . . . . 3

1.3 Algebraic $K$-Theory $\ldots \ldots \ldots \ldots$

2 The Algebraic Approach 9

2.1 The Construction of the Algebraic Invariant . . . . . . . . 9

2.2 Functoriality . . . . . . . . . . . . . . . . . . 12

2.3 Additive Properties . . . . . . . . . . . . . . . 18

3 The Geometric Approach $\quad 25$

3.1 The Definition of the Geometric Invariant . . . . . . . . . 25

3.2 An Illustrative Example . . . . . . . . . . . . . . . . . . 28

3.3 Functoriality . . . . . . . . . . . . . . . 32

3.4 The Induction Structure . . . . . . . . . . . . . . . . . 33

3.5 Properties of the Geometric Invariant . . . . . . . . . 34

4 Universality $\quad 47$

4.1 Notations and Results . . . . . . . . . . . . . . . . . 47

4.2 Proof of the Universal Property . . . . . . . . . . . . . . 53

5 Splitting Results $\quad 65$

5.1 An Equivalence Relation . . . . . . . . . . . . . . . 65

5.2 Definition of the Splitting Functors . . . . . . . . . . . 68

5.3 From Categories to Groupoids . . . . . . . . . . . . . . . 74

5.4 A Finer Splitting Result . . . . . . . . . . . . . . . . . 79

5.5 A Small Example . . . . . . . . . . . . . . . . . . 84

6 The Geometric Meaning of the Splitting Results 85

6.1 Reduction to Groups instead of Groupoids . . . . . . . . . . . 85

6.2 The Geometric Meaning of the Splitting Result . . . . . . . . 97

6.3 The Illustrative Example, continued . . . . . . . . . . . . . 102 
7 The Generalized Equivariant Lefschetz Invariant 103

7.1 The Target Group . . . . . . . . . . . . . . . . . . . 104

7.2 A Convenient Trace Map . . . . . . . . . . . . . . . 108

7.3 The Refined Equivariant Lefschetz Number . . . . . . . . . . 113

7.4 The Generalized Trace Map . . . . . . . . . . . . . . 116

7.5 The Generalized Equivariant Lefschetz Invariant . . . . . . . 121

7.6 Relation to the Equivariant Lefschetz Class . . . . . . . . 125

8 The Refined Equivariant Lefschetz Fixed Point Theorem 127

8.1 The Refined Orbifold Lefschetz Fixed Point Theorem . . . . . 127

8.2 The Local Equivariant Lefschetz Class . . . . . . . . . . . . 129

8.3 The Illustrative Example, yet continued . . . . . . . . . . . 139

9 Equivariant Nielsen Invariants $\quad 141$

9.1 The Nielsen Number . . . . . . . . . . . . . . . . . . . . . . . 141

9.2 Equivariant Nielsen Invariants . . . . . . . . . . . . . . . 142

9.3 Lower Bound Property . . . . . . . . . . . . . . . . . . . . . . 144

9.4 The $G$-Jiang Condition . . . . . . . . . . . . . . . . . 147

9.5 Converse of the Equivariant Lefschetz Fixed Point Theorem . 149 


\section{Introduction}

Algebraic topology is the study of topological spaces and continuous maps with algebraic methods. One extracts information from the topological world by translating it into the world of algebra. To topological spaces and continuous maps, one assigns algebraic invariants. One of the most important invariants of a space $X$ is the Euler characteristic $\chi(X)$. The analog when dealing with an endomorphism of a topological space is the Lefschetz number. It is defined as follows:

Definition. Let $X$ be a topological space with finite homology, i.e., such that only finitely many homology groups are nontrivial and all of these have finite rank, and let $f: X \rightarrow X$ be an endomorphism. Then the Lefschetz number of $f$ is defined to be

$$
L(f):=\sum_{i \geq 0}(-1)^{i} \operatorname{tr}\left(H_{i}(f): H_{i}(X ; \mathbb{Z}) \rightarrow H_{i}(X ; \mathbb{Z})\right) \in \mathbb{Z} .
$$

The Lefschetz number of the identity id $X$ is the Euler characteristic of $X$. We will work with CW-complexes in the sequel. For a finite CW-complex, the Lefschetz number can alternatively be calculated using the cellular chain complex. The reason that the Lefschetz number plays such an important role lies in the celebrated Lefschetz fixed point theorem.

Theorem. Let $X$ be a finite $C W$-complex. Then

$$
L(f) \neq 0 \Longrightarrow f \text { has a fixed point. }
$$

The Lefschetz fixed point theorem was first announced in 1923 by Lefschetz (for a restricted class of polyhedra), and details were published three years later [Lef26]. Extensions to larger classes of spaces appeared in the sequel. Fixed points play a prominent role in many areas of mathematics, in pure mathematics, but also in applied mathematics, for example in the study of dynamical systems. So it is no big surprise that numerous generalizations of the Lefschetz number and the Lefschetz theorem have been developed.

Generalizations include invariants which give more precise lower bounds on the number of fixed points such as the generalized Lefschetz invariant [Rei38, Wec41]. If we suppose $X$ connected for simplicity and ignore 
the basepoint, the generalized Lefschetz invariant $\lambda(f)$ is defined as follows:

$$
\lambda(f):=\sum_{i \geq 0}(-1)^{i} \operatorname{tr}\left(C_{i}^{c}(\tilde{f})\right) \in \mathbb{Z} \pi_{1}(X) / \sim,
$$

where $C^{c}(\widetilde{f})$ denotes the map induced by $f$ on the cellular chain complex of the universal covering space $\widetilde{X}$ and $\sim$ is a certain equivalence relation on $\pi_{1}(X)$, see Section 7.1. Invariants which capture all iterates of $f$ at the same time are also studied, like the Lefschetz zeta function [FH93, FH94, GN93, GN94], which is defined to be

$$
\zeta(f):=\sum_{n \geq 0} \frac{L\left(f^{n}\right)}{n} t^{n} \in \mathbb{Q} \llbracket t \rrbracket .
$$

It is tempting to search for a universal generalization of the Lefschetz number, i.e., an invariant which maps to all possible generalizations and still has the characteristic properties of the Lefschetz number, homotopy invariance and additivity. This has been successfully done by Lück [Lüc99]. He defines a universal functorial Lefschetz invariant $(U, u)$ for endomorphisms of finite $\mathrm{CW}$-complexes. Here, $U: \operatorname{End}\left(\mathcal{C} W_{\mathrm{fp}}\right) \rightarrow \mathcal{A} b$ is a functor which assigns an abelian group to each endomorphism $f: X \rightarrow X$. The function $u$ assigns an element $u(X, f) \in U(X, f)$ to the endomorphism $f$. See Section 3.1 for a description of this construction. Lück proves the following theorem:

Theorem. The pair $(U, u)$ is the universal functorial Lefschetz invariant for endomorphisms of finite $C W$-complexes.

The aim of this thesis is to generalize the construction of Lück to the equivariant case. We will be dealing with discrete groups $G$ and finite $G$-CWcomplexes. In many cases, group actions show up naturally. When there is a group action on a space, this gives additional structure, and we want to take this information into account in the construction of the invariant. The fixed point sets $X^{H}$ for subgroups $H \leq G$ play a role. We need to use clever ways of combining the information given at all fixed point sets.

Equivariant topology is an important area of topology. Many nonequivariant constructions in algebraic topology can be generalized to the equivariant case, for example the Euler characteristic. The Lefschetz number has also been generalized to the equivariant setting [LL89, LR03]. Sometimes group actions help to deal with spaces which cannot be approached otherwise. For example, if we have an infinite discrete group $G$ acting properly on a finite $G$-CW-complex $X$, the space $X$ seen as a CW-complex is infinite. If this space $X$ is a manifold, we cannot apply the Lefschetz fixed point theorem, but a $G$-equivariant analog [LR03, Theorem 0.2] can be applied.

One of the main results of this thesis lies in the definition of equivariant invariants which generalize the existing non-equivariant invariants in the 
right way. We want to mention the universal functorial equivariant Lefschetz invariant $\left(U_{G}^{\mathbb{Z}}(X, f), u_{G}^{\mathbb{Z}}(X, f)\right)$ defined in Chapters 2 and 3 and the generalized equivariant Lefschetz invariant $\left(\Lambda_{G}(X, f), \lambda_{G}(f)\right)$ defined in Chapter 7 . Some ideas going into these constructions are also interesting in their own right, for example the definition of the trace map in Section 7.2.

Another highlight are the splitting theorems, Theorems 5.3.1 and 5.4.2. They are obtained for all higher $K$-theory groups. It is likely that they have far-reaching applications concerning the $K$-theory of endomorphism categories.

Application of the splitting results to the geometric setting leads to a good understanding of the universal functorial equivariant Lefschetz invariant. Namely, it is given in terms of the maps $C^{c}\left(\left.\widetilde{f}\right|_{\widetilde{X}^{H}(x)},\left.\widetilde{f}\right|_{\widetilde{X}^{>H}(x)}\right)$ on the relative cellular chain complexes $C^{c}\left(\widetilde{X}^{H}(x), \widetilde{X}^{>H}(x)\right)$, for subgroups $H \leq G$ and points $x \in X$ with isotropy group $H$.

This result is applied to geometric questions concerning fixed points. The definition of $G$-equivariant Nielsen numbers in Chapter 9 is new for the case of infinite discrete groups $G$, as well as the corresponding theorems concerning their lower bound properties. The converse of the equivariant Lefschetz fixed point theorem, Theorem 9.5.3, is proved, which did not exist for infinite discrete groups $G$ and was not formulated in the elegant approach of Lück and Rosenberg [LR03] before.

Mastering technical difficulties is one of the main ingredients of this thesis, and those technical difficulties account for sometimes rather lengthy proofs. We hope the reader will still enjoy the ideas that lie behind.

This thesis consists of three blocks, given by Chapters 2 to 4 , Chapters 5 and 6, and Chapters 7 to 9 respectively. They are preceded by a brief introduction of some concepts used in the sequel.

The first block of this thesis concerns the construction of the universal functorial equivariant Lefschetz invariant. This is a generalization of the universal functorial Lefschetz invariant constructed by Lück [Lüc99, Chapters 1-4]. The algebraic foundations of our work are laid in Chapter 2. We construct an abelian group $U(R, \Gamma, \phi)$ for a commutative ring $R$, an EI-category $\Gamma$, i.e., a small category $\Gamma$ where all endomorphisms are isomorphisms, and an endofunctor $\phi: \Gamma \rightarrow \Gamma$. The group $U(R, \Gamma, \phi)$ is defined to be

$$
U(R, \Gamma, \phi):=K_{0}\left(\phi-\operatorname{end}_{\mathrm{ff} R \Gamma}\right),
$$

where $\phi$-end $\mathrm{ff}_{\mathrm{f} R}$ denotes the category of $\phi$-twisted endomorphisms of finite free $R \Gamma$-modules defined in Definition 2.1.2. We have a map $u$ mapping a finite free chain complex of $\phi$-twisted endomorphisms into $U(R, \Gamma, \phi)$. We show that $U(R, \Gamma, \phi)$ is a functorial additive invariant, meaning that it is 
functorial in $(\Gamma, \phi)$ and has an additive property with respect to short exact sequences.

In Chapter 3, the geometric setting is described. Starting with a discrete group $G$, a finite $G$-CW-complex $X$ and a $G$-equivariant cellular endomorphism $f: X \rightarrow X$, we introduce the cellular $R \Pi(G, X)$-chain complex $C^{c}(\widetilde{X})$. This is the equivariant analog of the cellular $R \pi_{1}(X, x)$-chain complex $C^{c}(\widetilde{X})$. The fundamental category $\Pi(G, X)$ defined in Definition 1.2.2 plays the role of the fundamental group. Using the algebraic definitions from Chapter 2 and setting $\phi:=\Pi(G, f)$, we define

$$
\begin{aligned}
U_{G}^{R}(X, f) & :=U(R, \Pi(G, X), \phi) \\
u_{G}^{R}(X, f) & :=u\left(C^{c}(\widetilde{f})\right) \in U_{G}^{R}(X, f) .
\end{aligned}
$$

We show that the pair $\left(U_{G}^{R}(X, f), u_{G}^{R}(X, f)\right)$ is a functorial equivariant Lefschetz invariant, i.e., that it is functorial, additive, $G$-homotopy invariant, invariant under $G$-homotopy equivalence and that it has an induction structure in $G$.

The climax of the first block is reached in Chapter 4, where universality of the invariant $\left(U_{G}^{\mathbb{Z}}(X, f), u_{G}^{\mathbb{Z}}(X, f)\right)$ among all functorial equivariant Lefschetz invariants is proved.

Theorem. For discrete groups $G$, the pair $\left(U_{G}^{\mathbb{Z}}, u_{G}^{\mathbb{Z}}\right)$ is the universal functorial equivariant Lefschetz invariant for the category of endomorphisms of finite proper $G$-CW-complexes.

The second block of this thesis concerns splitting results. The group $U(R, \Gamma, \phi)=K_{0}\left(\phi\right.$-end $\left.\mathrm{ff}_{\mathrm{f}} \Gamma\right)$ we are interested in is the 0 th $K$-group of a twisted endomorphism category. It is a rather complicated group, and we want to split it up into simpler parts which are easier to handle. We obtain splitting results for all $K$-groups of the category of $\phi$-twisted endomorphisms of finite free $R \Gamma$-modules, see Chapter 5 for notation.

Theorem. Let $(\Gamma, \phi)$ be an EI-category $\Gamma$ with order-respecting endofunctor $\phi$ such that every $W_{i} \in I_{\Gamma}$ is the colimit of its finite height subgroupoids. Then

$$
K\left(\phi-\operatorname{end}_{\mathrm{ff} R \Gamma}\right) \cong \bigoplus_{W_{i} \in I_{\Gamma}}\left(K\left(\phi_{T_{i}}-\operatorname{end}_{\mathrm{ff} R T_{i}}\right) \oplus K\left(\mathrm{ff} R Q_{i}\right)\right) .
$$

These splitting results are of independent interest in the study of algebraic $K$-theory of endomorphism categories. One can formulate them in the more general setting of $K$-theory of endomorphisms with coefficients in a bimodule [Bru05]. Numerous applications are possible, but they are beyond the scope of this thesis.

In Chapter 6, we describe the geometric meaning of the splitting results. The fixed point sets corresponding to the different subgroups $H \leq G$ come into play. We also show how to get back from groupoids to groups, thereby 
obtaining a description of our invariant in terms of the fundamental groups of the fixed point sets instead of their fundamental groupoids.

We obtain a statement expressing the universal functorial equivariant Lefschetz invariant $u_{G}^{\mathbb{Z}}(X, f)=\left[C^{c}(\widetilde{f})\right] \in K_{0}\left(\phi-\operatorname{end}_{\mathrm{ff} \mathbb{Z} \Pi(G, X)}\right)$ in terms of the maps $C^{c}\left(\left.\widetilde{f}\right|_{\widetilde{X}^{H}(x)},\left.\widetilde{f}\right|_{\widetilde{X}^{>H}(x)}\right)$ on the relative cellular chain complexes $C^{c}\left(\widetilde{X}^{H}(x), \widetilde{X}^{>H}(x)\right)$, for subgroups $H \leq G$ and objects $x \in \Pi(G, X)$ which correspond to points with isotropy group $H$. One arrives at the slogan: The $G$-equivariant information splits up into the relative information on the fixed point sets.

The last block of this thesis leads towards geometric applications. Chapters 7 and 8 are generalizations of results by Lück and Rosenberg [LR03, Sections 1-5]. In Chapter 7 we develop a generalized equivariant Lefschetz invariant $\left(\Lambda_{G}(X, f), \lambda_{G}(f)\right)$. It is an equivariant analog of the generalized Lefschetz invariant. A trace map $\operatorname{tr}_{G(X, f)}$ which maps the universal functorial equivariant Lefschetz invariant $\left(U_{G}^{\mathbb{Z}}(X, f), u_{G}^{\mathbb{Z}}(X, f)\right)$ to the generalized equivariant Lefschetz invariant $\left(\Lambda_{G}(X, f), \lambda_{G}(f)\right)$ is constructed.

The trace map is derived from the trace map $\operatorname{tr}_{R G}$ defined in Section 7.2 as a trace map on the group $\operatorname{ring} R G$, where $G$ is a discrete group extension with endomorphism as defined in Definition 7.2.1. The trace map $\operatorname{tr}_{R G}$ can be seen as a variation of a trace map $\operatorname{tr}_{(K \rightarrow H H)}$ between $K$-theory and Hochschild homology with coefficients in a bimodule. The map $\operatorname{tr}_{R G}$ is a restriction of $\operatorname{tr}_{(K \rightarrow H H)}$ to the direct summand containing fixed point information.

In Chapter 8, a generalized local equivariant Lefschetz class $\lambda_{G}^{\text {loc }}(f)$ is defined in terms of fixed point data. We prove the refined equivariant Lefschetz fixed point theorem, Theorem 8.2.7, which shows that the generalized equivariant Lefschetz invariant $\lambda_{G}(f) \in \Lambda_{G}(X, f)$ can be given as a sum of fixed point contributions.

Theorem. Let $G$ be a discrete group, let $M$ be a cocompact proper smooth $G$-manifold and let $f: M \rightarrow M$ be a $G$-equivariant endomorphism such that $\operatorname{Fix}(f) \cap \partial M=\emptyset$ and such that for every $x \in \operatorname{Fix}(f)$ the determinant of the map $\left(\operatorname{id}_{T_{x} M}-T_{x} f\right)$ is different from zero. Then

$$
\lambda_{G}(f)=\lambda_{G}^{l o c}(f)
$$

Based on the generalized equivariant Lefschetz invariant $\lambda_{G}(f)$ developed in Chapter 7, we introduce equivariant Nielsen invariants $N_{G}(f)$ and $N^{G}(f)$ in Chapter 9. They are equivariant analogs of the Nielsen number and give lower bounds for the number of fixed point orbits in the $G$-homotopy class of $f$. Under mild hypotheses, these lower bounds are sharp.

We use the equivariant Nielsen invariants to show that a $G$-equivariant endomorphism $f$ is $G$-homotopic to a fixed point free $G$-map if the generalized equivariant Lefschetz invariant $\lambda_{G}(f)$ is zero. 
Theorem. Let $G$ be a discrete group. Let $X$ be a cocompact proper smooth $G$-manifold satisfying the standard gap hypotheses. Let $f: X \rightarrow X$ be a $G$-equivariant endomorphism. Then the following holds:

If $\lambda_{G}(f)=0$, then $f$ is $G$-homotopic to a fixed point free $G$-map.

Finally, we prove a converse of the equivariant Lefschetz fixed point theorem: If $X$ is a $G$-Jiang space as defined in Definition 9.4.2, then $L_{G}(f)=$ 0 implies that $f$ is $G$-homotopic to a fixed point free map. Here, $L_{G}(f)$ is the equivariant Lefschetz class defined in Definition 7.6.1 [LR03, Definition 3.6], the equivariant analog of the Lefschetz number.

I thank my advisor Prof. Wolfgang Lück for letting me be part of his great algebraic topology group. I want to thank the members of this group for their friendly welcome and continuous support, in particular Tilman Bauer, Joachim Grunewald, Michael Joachim, Holger Reich, Juliane Sauer, Roman Sauer, Marco Schmidt and Clara Strohm. Special thanks go to Clara Strohm and Marco Varisco for their help with computer and IATEX problems. Most of all, I want to thank Marco Schmidt for his friendship and constant friendly smile, which was wonderful in difficult times.

I am thankful to Morten Brun for intense mathematical discussions, in particular concerning the splitting result in Chapter 5. I also thank Dirk Schütz for numerous thorough discussions of the trace map and the fixed point information in Chapters 7 and 9. It was a pleasure to find you two interested in this thesis.

Deepest thanks go to my family for their love and support, as well as to Elmar. Thank you for being there for me. 


\section{Chapter 1}

\section{Motivation and Basic Concepts}

In this chapter, we introduce the most important notions used in the sequel. We start with the classical Lefschetz invariant and the well-known Lefschetz fixed point theorem - after all, this is the basis of all research on Lefschetz invariants. We also introduce the technical tools needed for our generalization of the classical concepts. This is on the one hand the language of modules over categories, on the other hand that of $K$-theory.

\subsection{The Lefschetz Number}

The Lefschetz number is a very powerful invariant in algebraic topology, with far-reaching applications in many areas of mathematics. It is associated to an endomorphism of a topological space $X$.

Definition 1.1.1. Let $X$ be a topological space with finite homology, i.e., such that only finitely many homology groups are nontrivial and all of these have finite rank, and let $f: X \rightarrow X$ be an endomorphism. Then the Lefschetz number of $f$ is defined to be

$$
L(f):=\sum_{i \geq 0}(-1)^{i} \operatorname{tr}\left(H_{i}(f): H_{i}(X ; \mathbb{Z}) \rightarrow H_{i}(X ; \mathbb{Z})\right) \in \mathbb{Z} .
$$

The Lefschetz number incorporates the Euler characteristic of the space $X$. Namely, in the particular case where $f$ is the identity map of $X$ we have

$$
L\left(\operatorname{id}_{X}\right)=\chi(X) .
$$

If $X$ is a finite CW-complex and $f: X \rightarrow X$ is a cellular endomorphism, we can calculate the Lefschetz number by using the cellular chain complex of $X$. 
Lemma 1.1.2. Let $X$ be a finite $C W$-complex and let $f: X \rightarrow X$ be a cellular endomorphism. Then

$$
L(f)=\sum_{i \geq 0}(-1)^{i} \operatorname{tr}\left(C_{i}^{c}(f): C_{i}^{c}(X ; \mathbb{Z}) \rightarrow C_{i}^{c}(X ; \mathbb{Z})\right) \in \mathbb{Z} .
$$

This is the basis of the generalizations we have in mind. The relevance of the Lefschetz number lies in the Lefschetz fixed point theorem:

Theorem 1.1.3. Let $X$ be a finite $C W$-complex. Then

$$
L(f) \neq 0 \Longrightarrow f \text { has a fixed point. }
$$

So the Lefschetz number gives a purely algebraic way of seeing whether a map has a fixed point. This, in turn, is very important in many areas of mathematics, but we will not go into the applications here. Instead, we ask ourselves for refinements of the Lefschetz number. See [Bro71] for an introduction to the Lefschetz number, the Lefschetz fixed point theorem and related topics.

If we are given a discrete group $G$ and a $G$-CW-complex $X$ with an equivariant endomorphism $f: X \rightarrow X$, then we want to consider analogous invariants which take the group action into account.

We will be concerned with spaces with an action of a discrete group $G$. In this context, CW-complexes are replaced with $G$-CW-complexes, and we give their definition here. See Lück [Lüc89, Chapter 1] for more details on $G$-CW-complexes and their properties.

Definition 1.1.4. Let $G$ be a discrete group. A $G$-CW-complex $X$ is defined inductively: The 0-skeleton is defined by

$$
X_{0}:=\coprod_{j \in J_{0}} G / H_{0, j} \times D_{j}^{0} .
$$

The $i$-skeleton $X_{i}$ of $X$ is defined to be the pushout

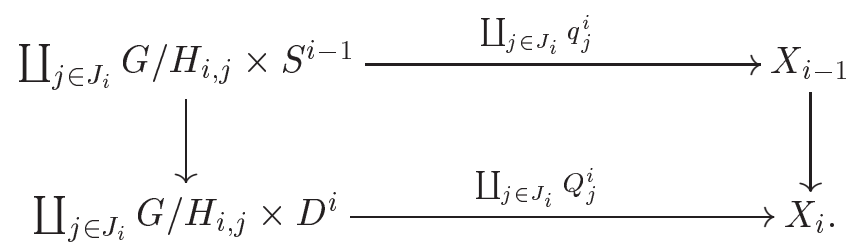

Here the $H_{i, j} \leq G$ are subgroups of $G$. We define $X:=\bigcup_{i \geq 0} X_{i}$. The $G$-CWcomplex $X$ has a natural left $G$-action. The subset $Q_{j}^{i}\left(G / H_{i, j} \times \stackrel{D}{D}^{i}\right) \subseteq X$ is called an $i$-cell of $X$. The $G$-CW-complex $X$ is called finite when there are only a finite number of cells. It is called proper when all subgroups $H_{i, j} \leq G$ appearing are finite.

One has the notion of $G$-equivariant cellular maps, and one can also define $G$-cellular approximations [tD87, II.2]. We will work with $G$-CWcomplexes in the sequel whenever we are in the geometric setting. 


\subsection{Modules over Categories}

This section is designed to establish the language concerning modules over categories we will need later on. All its contents are taken from [Lüc89]. The reader is referred to [Lüc89] for a more exhaustive presentation, and to [DL98] for a nice introduction to this modern language. See [Bre72] and [tD87] for basic facts concerning transformation groups.

We will work with EI-categories throughout this thesis. So we start with their definition:

Definition 1.2.1. A small category is a category $\Gamma$ where the class $\mathrm{Ob} \Gamma$ of objects is a set. An EI-category is a small category such that any endomorphism $f: x \rightarrow x$ in $\Gamma$ is an isomorphism.

Examples include groups considered as categories and groupoids. The only morphisms appearing there are isomorphisms. Another example is the orbit category of a discrete group $G$. For us, the most important example of an EI-category is the fundamental category of a topological space $X$ with an action of a discrete group $G$ [Lüc89, Definition 8.15].

Definition 1.2.2. Let $G$ be a discrete group, and let $X$ be a $G$-space. Then the fundamental category $\Pi(G, X)$ is the following category:

- The objects $\mathrm{Ob}(\Pi(G, X))$ are $G$-maps $x: G / H \rightarrow X$, where the $H \leq$ $G$ are subgroups. (We often abbreviate an object by $x(H)$ or $x$.)

- The morphisms $\operatorname{Mor}(x(H), y(K))$ are pairs $(\sigma,[w])$, where

- $\sigma$ is a $G$-map $\sigma: G / H \rightarrow G / K$

- $[w]$ is a homotopy class of $G$-maps $w: G / H \times I \rightarrow X$ relative $G / H \times \partial I$ such that $w_{1}=x$ and $w_{0}=y \circ \sigma$. (We often abbreviate a morphism by $(\sigma, w)$.)

Composition of the morphism $(\sigma,[w]): x(H) \rightarrow y(K)$ and the mor$\operatorname{phism}(\tau,[v]): y(K) \rightarrow z(L)$ is given by $(\tau,[v]) \circ(\sigma,[w])=\left(\tau \circ \sigma,\left[\sigma^{*}(v) *\right.\right.$ $w])$, where $\sigma^{*}(v)=v \circ(\sigma \times \mathrm{id}): G / H \times I \rightarrow X$ and $*$ denotes the composition of paths.

The fundamental category is a combination of the orbit category of $G$ and the fundamental groupoid of $X$. If $X$ is a point, then the fundamental category is just the orbit category of $G$, whereas when $G$ is the trivial group, the definition reduces to the definition of the fundamental groupoid of $X$.

The orbit category of a discrete group $G$ is defined to be the category with objects homogeneous spaces $G / H$ and with morphisms $G$-maps $\sigma: G / H \rightarrow G / K$. We know that $G$-maps $\sigma: G / H \rightarrow G / K$ are given by right translations with elements $g \in G$ which have the property that $g H^{-1} \leq K$, 
namely $R_{g}: G / H \rightarrow G / K, \widetilde{g} H \mapsto \widetilde{g} g K$ [tD87, Proposition 1.14]. One sees that $R_{g k}=R_{g}$ for all $k \in K$.

The Weil group $W H:=N_{G} H / H$ of $H$ is defined to be the normalizer of $H$ in $G$ modulo $H$ itself. There is an isomorphism between $W H^{o p}$ and $\operatorname{Aut}(G / H)$ given by $W H^{o p} \rightarrow \operatorname{Aut}(G / H), g H \mapsto R_{g^{-1}}$. Here the upper index op denotes the opposite group.

Very important to us will be the automorphism group $\operatorname{Aut}(x)$ of an element $(x: G / H \rightarrow X) \in \Pi(G, X)$. It is a group extension [Lüc89, 8.23]

$$
1 \rightarrow \pi_{1}\left(X^{H}(x), x\right) \rightarrow \operatorname{Aut}(x) \rightarrow W H_{x}^{o p} \rightarrow 1 .
$$

Here the fixed point set $X^{H}:=\{x \in X \mid h . x=x$ for all $h \in H\}$ is the set of points which are fixed under the action of the subgroup $H \leq G$ and $X^{H}(x)$ denotes the connected component of $X^{H}$ containing $x(1 H)$. We often identify the object $(x: G / H \rightarrow X) \in \Pi(G, X)$ with the point $x(1 H) \in X$. The group $\pi_{1}\left(X^{H}(x), x\right)$ is the fundamental group of $X^{H}(x)$ with basepoint $x$. The action of $G$ on $X$ induces an action of the Weil group $W H$ on $X^{H}$. The group $W H_{x} \leq W H$ denotes the subset of $W H$ which fixes the connected component $X^{H}(x)$. We will mostly omit the index op in the sequel.

In the context of generalized Lefschetz invariants, the fundamental group plays a prominent role, and this role is assumed by the fundamental category in the $G$-equivariant setting.

When one works with a CW-complex $X$, the classical way to take the fundamental group action into account is to consider the cellular chain complex of the universal covering space $\widetilde{X}$. The covering space $\widetilde{X}$ carries a $\pi_{1}(X, x)$ action (when $x$ is a chosen basepoint), and this induces a $\mathbb{Z} \pi_{1}(X, x)$-chain complex structure on the cellular chain complex $C^{c}(\widetilde{X}, \widetilde{x})$.

When there is a group $G$ acting on $X$, then there is more information given, encoded in the different fixed point sets $X^{H}$ for subgroups $H \leq G$. So we need to consider the fundamental groups of the fixed point sets, and the group actions on them induced by $G$. How can we assemble all this information?

The natural object combining the $G$-action information and the fundamental group information is the fundamental category. The notion of a $\mathbb{Z} \pi_{1}(X, x)$-module does not seem to leave room for a generalization from $\pi_{1}(X, x)$ to $\Pi(G, X)$. But one can view a $\mathbb{Z} \pi_{1}(X, x)$-module as a functor from the category $\widehat{\pi_{1}(X, x)}$ that has one object and all elements of $\pi_{1}(X, x)$ as morphisms to the category $\mathcal{A} b$ of abelian groups. This is an equivalent notion, and this can be generalized: We look at functors from $\Pi(G, X)$ to abelian groups.

The above discussion motivates the following definition of a module over a category, which lies at the heart of all further considerations [Lüc89, 9.4]: 
Definition 1.2.3. Let $R$ be a commutative ring, and let $\Gamma$ be a small category. An $R \Gamma$-module is a functor

$$
M: \Gamma \rightarrow R \text {-Mod. }
$$

A homomorphism of $R \Gamma$-modules is a natural transformation of functors.

From now on, $R$ denotes a commutative ring. An $R \Gamma$-module corresponds to a left module if the functor is covariant, to a right module if the functor is contravariant.

Convention 1.2.4. All our modules are contravariant functors unless explicitly stated otherwise.

The category of $R \Gamma$-modules is an abelian category, the structure is inherited from the category of $R$-modules. One defines injective and projective modules as usual. Most constructions known for modules over (group) rings can be generalized to modules over categories. We collect those that will be most important to us. We will often need the tensor product of two $R \Gamma$-modules [Lüc89, 9.13].

Definition 1.2.5. Let $M$ be a contravariant functor $\Gamma \rightarrow R$-Mod and let $N$ be a covariant functor $\Gamma \rightarrow R$-Mod. Then their tensor product $M \otimes_{R \Gamma} N$ is defined to be the $R$-module

$$
M \otimes_{R \Gamma} N:=\bigoplus_{x \in \Gamma} M(x) \otimes_{R} N(x) / \sim,
$$

where the equivalence relation is generated by $M(f)(m) \otimes n \sim m \otimes N(f)(n)$ for $m \in M(y), n \in M(x), f \in \operatorname{Mor}_{\Gamma}(x, y)$ and $x, y \in \Gamma$.

We will also work constantly with the two covariant functors induction and restriction [Lüc89, 9.15]:

Definition 1.2.6. Let $F: \Gamma_{1} \rightarrow \Gamma_{2}$ be a covariant functor. Denote by $R \Gamma_{2}(? ?, F(?))$ the $R \Gamma_{1}-R \Gamma_{2}$-bimodule $\left(x_{1}, x_{2}\right) \mapsto R \operatorname{Mor}_{\Gamma_{2}}\left(x_{2}, F\left(x_{1}\right)\right)$. (An $R \Gamma_{1}-R \Gamma_{2}$-bimodule is a covariant functor $M: \Gamma_{1} \times \Gamma_{2}^{o p} \rightarrow R$-Mod.) This bimodule is used for defining induction with $F$. Restriction with $F$ is defined using $R \Gamma_{2}(F(?), ? ?)$. We set

$$
\begin{aligned}
\operatorname{ind}_{F}: \quad R \Gamma_{1} \text {-Mod } & \rightarrow R \Gamma_{2} \text {-Mod } \\
M & \mapsto M \otimes_{R \Gamma_{1}} R \Gamma_{2}(? ?, F(?)) \\
\operatorname{res}_{F}: \quad R \Gamma_{2} \text {-Mod } & \rightarrow R \Gamma_{1} \text {-Mod } \\
M & \mapsto M \otimes_{R \Gamma_{2}} R \Gamma_{2}(F(?), ? ?) .
\end{aligned}
$$

We often denote $M \otimes_{R \Gamma_{2}} R \Gamma_{2}(F(?), ? ?)$ by $M \circ F$. Induction and restriction are adjoint functors [Lüc89, 9.22]. 
Lemma 1.2.7. Given a covariant functor $F: \Gamma_{1} \rightarrow \Gamma_{2}$, the functors $\operatorname{ind}_{F}$ and $\operatorname{res}_{F}$ are adjoint.

Another important notion is that of a free module over a category [Lüc89, Definition 9.17]:

Definition 1.2.8. Let a set $B$ and a $\operatorname{map} \beta: B \rightarrow \mathrm{Ob} \Gamma$ be given. An $R \Gamma$ module $M$ with $b \in M(\beta(b))$ for all $b \in B$ is free with base $B$ if for any $R \Gamma$-module $N$ and any map $f: B \rightarrow N$ with $f(b) \in N(\beta(b))$ for all $b \in B$ there is exactly one $R \Gamma$-homomorphism $F: M \rightarrow N$ extending $f$.

A model for the free $R \Gamma$-module with base $B$ is

$$
R \Gamma(B)=\bigoplus_{b \in B} R \Gamma(?, \beta(b)) .
$$

We call a free module $M$ with base $B$ finite if the set $B$ is finite.

In [Lüc89, 9.24] we find the following statement:

Remark 1.2.9. The induction functor respects "direct sum", "finitely generated", "free" and "projective".

We have several functors relating an $R \Gamma$-module $M$ to modules over the group rings $R \operatorname{Aut}(x)$. For brevity of notation, we often write $R[x]$ for $R \operatorname{Aut}(x)$, for $x \in \Gamma$. Since $\Gamma$ is an EI-category, there is a natural partial ordering $\leq$ on the set Is $\Gamma$ of isomorphism classes $\bar{x}$ of objects in $\Gamma$ given by

$$
\bar{x} \leq \bar{y} \Leftrightarrow \operatorname{Mor}_{\Gamma}(x, y) \neq \emptyset .
$$

We define the following functors [Lüc89, 9.26-9.29]:

Definition 1.2.10. Let $\Gamma$ be an EI-category, and let $x$ be an object of $\Gamma$. We define the splitting functor $S_{x}$, the restriction functor $\mathrm{RES}_{x}$, the extension functor $E_{x}$ and the inclusion functor $I_{x}$ by the following maps on modules over a category.

$$
\begin{aligned}
\text { Splitting functor } S_{x}: \quad R \Gamma-\operatorname{Mod} & \rightarrow R[x] \text {-Mod } \\
M & \mapsto M(x) / M(x)_{s}, \\
\text { Restriction functor } \operatorname{RES}_{x}: \quad R \Gamma \text {-Mod } & \rightarrow R[x] \text {-Mod } \\
M & \mapsto M(x), \\
\text { Extension functor } E_{x}: \quad R[x] \text {-Mod } & \rightarrow R \Gamma \text {-Mod } \\
M & \mapsto M \otimes_{R[x]} R \operatorname{Mor}_{\Gamma}(?, x), \\
\text { Inclusion functor } I_{x}: \quad R[x]-\operatorname{Mod} & \rightarrow R \Gamma-\operatorname{Mod} \\
M & \mapsto I_{x} M .
\end{aligned}
$$

Here $M(x)_{s}$ is the singular $R[x]$-module at $x$ defined by

$$
M(x)_{s}:=\langle\{\operatorname{Im}(M(f): M(y) \rightarrow M(x)) \mid f: x \rightarrow y, f \text { non-isom. }\}\rangle \subseteq M(x)
$$


and the $R \Gamma$-module $I_{x} M$ is defined by

$$
I_{x} M(y)= \begin{cases}M(x) \otimes_{R[x]} R \operatorname{Mor}_{\Gamma}(y, x) & \text { if } \bar{y}=\bar{x} \\ \{0\} & \text { if } \bar{y} \neq \bar{x} .\end{cases}
$$

The functors $S_{x}$ and $I_{x}$ are adjoint to each other, as well as the functors $E_{x}$ and $\operatorname{RES}_{x}$ [Lüc89, 9.31]. Note that the restriction functor $\mathrm{RES}_{x}$ is just the restriction with the inclusion $i: \operatorname{Aut}(x) \rightarrow \Gamma$, and that $E_{x}$ is induction with $i$. The splitting functor can be viewed as $M \mapsto M \otimes_{R \Gamma} B$, where $B$ is defined to be the $R \Gamma-R[x]$-bimodule given by

$$
\Gamma \rightarrow R[x]-\operatorname{Mod}, \quad y \mapsto \begin{cases}R \operatorname{Mor}_{\Gamma}(x, y) & \text { if } \bar{x}=\bar{y} \\ \{0\} & \text { if } \bar{x} \neq \bar{y}\end{cases}
$$

Remark 1.2.11. The functors $S_{x}$ and $E_{x}$ respect "direct sum", "finitely generated", "free" and "projective". We also know that $S_{x} \circ E_{x}$ is naturally equivalent to the identity and that $S_{y} \circ E_{x}$ is zero for $\bar{x} \neq \bar{y}$ [Lüc89, 9.31].

\subsection{Algebraic $K$-Theory}

Another important concept used in this thesis is algebraic $K$-theory, and we shall give a brief outline of some definitions and results used in the sequel. For more complete accounts of $K$-theory, there are numerous references. Classical references for algebraic $K$-theory are [Bas68] and [Mil71], others include [Kar78] and [Ros94]. This thesis essentially only uses $K_{0}$ of a suitable category, but the results obtained in Chapters 5 and 6 work for all algebraic $K$-groups. We are interested in $K$-theory of exact categories, and we proceed to give the definition of an exact category.

Definition 1.3.1. A category $\mathcal{C}$ is called additive if it satisfies

- All finite coproducts exist in $\mathcal{C}$.

- For all objects $A, B \in \mathcal{C}$ the set $\operatorname{Mor}(A, B)$ is an abelian group.

- Composition of morphisms is a bilinear map.

- There is a distinguished zero object 0 such that $\operatorname{Mor}(A, 0)=0$ and $\operatorname{Mor}(0, A)=0$ for each $A \in \mathrm{ObC}$.

- There is a binary operation $\oplus$ which is both the categorical product and the categorical coproduct.

Definition 1.3.2. An abelian category is an additive category in which every morphism has a kernel and a cokernel, and in which every monomorphism is a kernel and every epimorphism is a cokernel. 
An abelian category has a notion of exact sequences $0 \rightarrow A \stackrel{i}{\rightarrow} B \stackrel{p}{\rightarrow}$ $C \rightarrow 0$. Loosely speaking, such a sequence is exact if the image of one arrow is the kernel of the next. A good reference for abelian categories is [Mac71].

Definition 1.3.3. An exact category is a full additive subcatecory $\mathcal{D}$ of an abelian category $\mathcal{C}$ which fulfills:

- The category $\mathcal{D}$ is closed under extensions, i.e., if

$$
0 \rightarrow D_{1} \rightarrow D \rightarrow D_{2} \rightarrow 0
$$

is an exact sequence in $\mathcal{C}$ and $D_{1}, D_{2} \in \mathrm{Ob} \mathcal{D}$, then $D \in \mathrm{Ob} \mathcal{D}$.

- The category $\mathcal{D}$ has a small skeleton, i.e., $\mathcal{D}$ has a full subcategory $\mathcal{D}_{0}$ which is small and for which the inclusion $\mathcal{D}_{0} \rightarrow \mathcal{D}$ is an equivalence.

The exact sequences in such a category are defined to be the exact sequences in the ambient category $\mathcal{C}$ involving only objects (and morphisms) chosen from $\mathcal{D}$.

In contrast to the abelian case, the notion of exactness depends on the embedding of $\mathcal{D}$ in $\mathcal{C}$. In particular, an additive category may have more than one structure as an exact category. One can always endow an additive category with the exact structure given by split exact sequences [Swa95, Section 1]. A sequence $1 \rightarrow D_{1} \stackrel{i}{\rightarrow} D \stackrel{p}{\rightarrow} D_{2} \rightarrow 0$ splits if there exists a morphism $s: D_{2} \rightarrow D$ such that $p s=\operatorname{id}_{D_{2}}$.

One example of an exact category is the category of finitely generated projective modules over a ring, one obtains algebraic $K$-theory of the ring as the algebraic $K$-theory of this category. In our context, it is important to keep the following example of an exact category in mind:

Example 1.3.4. Let $\Gamma$ be an EI-category. Then the category of finitely generated free $R \Gamma$-modules with the exact sequences given by split exact sequences is an exact category [Lüc89, p. 183].

We will mostly be concerned with $K_{0}$ of exact categories, so we give the explicit definition here.

Definition 1.3.5. Let $\mathcal{D}$ be an exact category with small skeleton $\mathcal{D}_{0}$. We define $K_{0}(\mathcal{D})$ to be the free abelian group on $\mathrm{Ob} \mathcal{D}_{0}$ modulo the relations

- $[D]=\left[D^{\prime}\right]$ if there is an isomorphism $D \stackrel{\sim}{\rightarrow} D^{\prime}$ in $\mathcal{D}$.

- $[D]=\left[D_{1}\right]+\left[D_{2}\right]$ if there is a short exact sequence

$$
0 \rightarrow D_{1} \rightarrow D \rightarrow D_{2} \rightarrow 0 \text { in } \mathcal{D} \text {. }
$$

A good reference for $K_{0}$ is [Swa95]. The $K_{1}$-groups can be defined in terms of automorphisms. From then on one needs a general theory. Much work has gone into the definition of higher K-groups of exact categories that was completed by Quillen [Qui73]. We will not give the definition here, it can be found in the references cited. 


\section{Chapter 2}

\section{The Algebraic Approach}

We begin our work with purely algebraic considerations. Given a commutative ring $R$, we define an abelian group $U(R, \Gamma, \phi)$ for any EI-category $\Gamma$ with endofunctor $\phi$. It is defined as $K_{0}$ of the category of $\phi$-twisted endomorphisms which will be defined in Definition 2.1.2.

One may already keep in mind that in the geometric context we are given a discrete group $G$, a finite free $G$-CW-complex $X$ and a $G$-equivariant endomorphism $f: X \rightarrow X$. Then the fundamental category $\Pi(G, X)$ plays the role of $\Gamma$ and $\phi$ is induced by $f$.

We proceed to show functoriality of $U(R, \Gamma, \phi)$ in $(\Gamma, \phi)$. This is needed to prove functoriality of the geometric invariant in $(X, f)$ and to define an induction structure in $G$.

We finally analyze properties of $U(R, \Gamma, \phi)$, in particular we obtain the statement that $U(R, \Gamma, \phi)$ together with a certain function $u$ is a universal additive invariant in the sense of Definitions 2.3.1 and 2.3.2 on the category of $\phi$-twisted endomorphisms.

\subsection{The Construction of the Algebraic Invariant}

Let $\Gamma$ be an EI-category as defined in Definition 1.2.1 and let $\phi: \Gamma \rightarrow \Gamma$ be a functor. Remember from Definition 1.2.3 that an $R \Gamma$-module $M$ is a contravariant functor $M: \Gamma \rightarrow R$-Mod, where $R$ is a commutative ring. Then $M \circ \phi: \Gamma \rightarrow R$-Mod is also an $R \Gamma$-module.

Definition 2.1.1. Let $R$ be a commutative ring, let $\Gamma$ be an EI-category and let $\phi: \Gamma \rightarrow \Gamma$ be an endofunctor. We define a $\phi$-endomorphism $g$ of an $R \Gamma$-module $M$ to be a natural transformation $g: M \rightarrow M \circ \phi$.

We now look at the category $\phi$-end $\mathrm{fff}_{\mathrm{ff}}$ of $\phi$-endomorphisms of finite free $R \Gamma$-modules.

Definition 2.1.2. Let $R$ be a commutative ring, let $\Gamma$ be an EI-category and let $\phi: \Gamma \rightarrow \Gamma$ be an endofunctor. Then the category $\phi$-end $\mathrm{ff}_{\mathrm{f} R \Gamma}$ of $\phi$-twisted 
endomorphisms of finite free $R \Gamma$-modules is defined as follows: Objects are natural transformations

$$
g: M \rightarrow M \circ \phi
$$

where $M$ is a finite free $R \Gamma$-module. Morphisms from $g: M \rightarrow M \circ \phi$ to $h: N \rightarrow N \circ \phi$ are natural transformations $\tau: M \rightarrow N$ such that the diagram

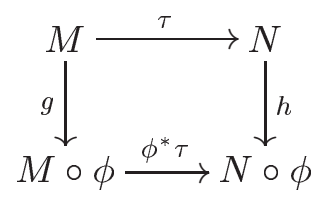

commutes, i.e., such that for all $x \in \mathrm{Ob} \Gamma$ the diagram

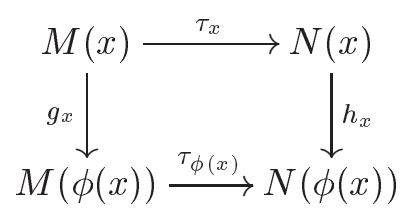

commutes and for all morphisms $(w: x \rightarrow y)$ in $\Gamma$ the diagram

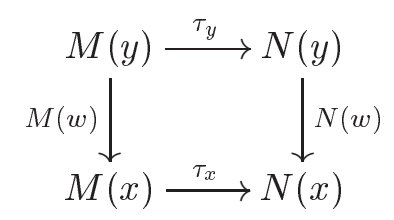

commutes. We write $\tau: g \rightarrow h$.

The category $\phi$-end $\mathrm{ff}_{\mathrm{f} \Gamma}$ is an exact category. This is true for the category of finite free $R \Gamma$-modules, Example 1.3.4, and all necessary properties are inherited by $\phi-\operatorname{end}_{\mathrm{ff} R \Gamma}$. (Recall that morphisms are just a subset of the morphisms of the category ff $R \Gamma$ of finite free $R \Gamma$-modules, closed under composition.) An exact sequence $0 \rightarrow g_{0} \stackrel{i}{\rightarrow} g \stackrel{p}{\rightarrow} g_{1} \rightarrow 0$ is given by a commutative diagram with exact rows

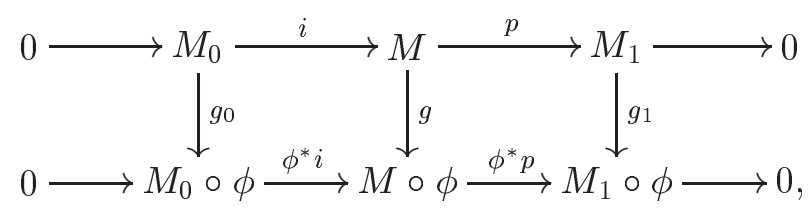

where $M_{0}, M$ and $M_{1}$ are finite free $R \Gamma$-modules.

The isomorphism classes of objects of $\phi$-end $\mathrm{ff}_{R \Gamma}$ form a set since every finite free $R \Gamma$-module $M$ is isomorphic to one of the form $\bigoplus_{b \in B} R \Gamma(?, \beta(b))$, where $B$ is a finite set and $\beta: B \rightarrow \mathrm{Ob} \Gamma$ a map, see Definition 1.2.8. A $\phi-$ endomorphism $g$ of $M$ induces a unique $\phi$-endomorphism of this module and vice-versa. Thus every isomorphism class of objects of $\phi$-end $\mathrm{ff} R \Gamma_{\Gamma}$ has a 
representative which can be written as a matrix, and all these matrices form a set. (Note that $\mathrm{Ob} \Gamma$ is a set since $\Gamma$ is an EI-category.) So one can apply the functor $K_{0}$ (see Definition 1.3.5) to the category $\phi$-end $\mathrm{fff}_{\mathrm{f}} \Gamma$.

Definition 2.1.3. Let $R$ be a commutative ring, let $\Gamma$ be an EI-category and let $\phi: \Gamma \rightarrow \Gamma$ be an endofunctor. We define

$$
U(R, \Gamma, \phi):=K_{0}\left(\phi-\operatorname{end}_{\mathrm{ff} R \Gamma}\right) .
$$

This is an abelian group. Elements in $U(R, \Gamma, \phi)$ are represented by $\phi-$ endomorphisms $g: M \rightarrow M \circ \phi$, the element represented by $g$ is denoted by $[g]$. One can also define chain complexes of $\phi$-endomorphisms of finite free $R \Gamma$-modules. These are given by ladder diagrams

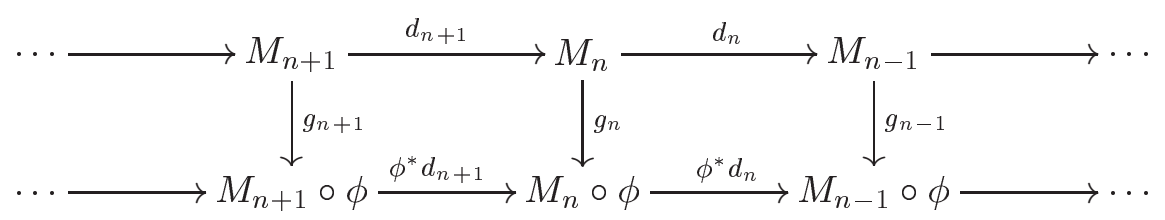

with $d_{n} \circ d_{n+1}=0$ for all $n$ (and where every square commutes).

One maps a finite free chain complex of $\phi$-endomorphisms into $U(R, \Gamma, \phi)$ by

$$
u:\left\{\cdots \rightarrow g_{n} \rightarrow g_{n-1} \rightarrow \cdots\right\} \mapsto \sum_{n \in \mathbb{Z}}(-1)^{n}\left[g_{n}\right] \in U(R, \Gamma, \phi) .
$$

Remark 2.1.4. One could also use the category $\phi$-end $\mathrm{d}_{\mathrm{ff} R \Gamma \text {-ch }}$ of chain complexes of $\phi$-endomorphisms of finite free $R \Gamma$-modules in the definition of the group $U(R, \Gamma, \phi)$. The chain complex category is equivalent to the module category [CP97], so the $K$-theory groups are the same. The category of chain complexes has several technical advantages, for example the existence of a cylinder functor.

Remark 2.1.5. Since $\phi-\operatorname{end}_{\mathrm{ff} R \Gamma}$ is an exact category, we can define all higher K-groups of this category. We will mostly be interested in $K_{0}$ since that is where our invariant lives. But when possible we will treat all higher $\mathrm{K}$-groups at once, for example in the splitting results in Chapters 5 and 6 .

Remark 2.1.6. When $\Gamma$ is a group $G$ viewed as a category, then the category $\phi$-end $\mathrm{ff}_{\mathrm{f}} \Gamma$ can be seen as the category of $R G$-endomorphisms with coefficients in the bimodule $M_{\phi}=R G(\phi(?), ? ?)$. The $K$-theory of endomorphisms with coefficients in a bimodule is an object of active research. There is work in progress by Brun and McCarthy stating that for a ring $S$ and a bimodule $M$ the group $\widetilde{K}_{0}(\operatorname{End}(S ; M))$ is a dense subset in the ring of twisted Witt vectors [Bru04]. This is a generalization of the fact that for a commutative ring $A$ the group $\widetilde{K}_{0}(\operatorname{End}(A))$ lies dense in the ring of Witt vectors of $A$ [Alm74]. 
The results of Brun and McCarthy give a description of the abelian group $U(R, \Gamma, \phi)$ when $\Gamma$ is a group $G$ viewed as a category, and thus of the target group of the universal functorial Lefschetz invariant [Lüc99]. Interpreting them in the setting of $R \Gamma$-modules or using the splitting developed in Chapter 5 one can describe the target group of the universal functorial equivariant Lefschetz invariant which is constructed in this thesis. On the contrary, the universal functorial (equivariant) Lefschetz invariant gives an interesting element living in these groups. It is possible to extend results here to the more general setting of categories of endomorphisms with coefficients in a general bimodule instead of just using the specific bimodule given by restriction with $\phi$, for example the splitting results from Chapter 5 [Bru05]. This could have very interesting applications, but these are beyond the scope of this thesis.

\subsection{Functoriality}

If we have a covariant functor $H: \Gamma_{1} \rightarrow \Gamma_{2}$ of EI-categories with endofunctors $\phi$ and $\psi$ such that the diagram

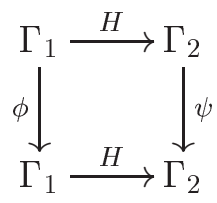

commutes, do we obtain a group homomorphism $U(R, H): U\left(R, \Gamma_{1}, \phi\right) \rightarrow$ $U\left(R, \Gamma_{2}, \psi\right)$ ?

The answer is yes, we construct such a group homomorphism using the covariant functor "induction with $H$ " defined in Definition 1.2 .6 by

$$
\begin{aligned}
\operatorname{ind}_{H}: R \Gamma_{1} \text {-Mod } & \rightarrow R \Gamma_{2} \text {-Mod } \\
M & \mapsto M \otimes_{R \Gamma_{1}} R \Gamma_{2}(? ?, H(?)) .
\end{aligned}
$$

We first construct an exact functor $H_{*}: \phi-\operatorname{end}_{\mathrm{ff} R \Gamma_{1}} \rightarrow \psi-\operatorname{end}_{\mathrm{ff} R \Gamma_{2}}$, then we take the induced map in $K_{0}$. Given a $\phi$-endomorphism $g: M \rightarrow M \circ \phi$ of a finite free $R \Gamma_{1}$-module $M$, we want to map $M$ to $\operatorname{ind}_{H} M$. Thus we need to find a $\psi$-endomorphism $H_{*}(g): \operatorname{ind}_{H} M \rightarrow\left(\operatorname{ind}_{H} M\right) \circ \psi$. The morphism $\operatorname{ind}_{H}(g): \operatorname{ind}_{H} M \rightarrow \operatorname{ind}_{H}(M \circ \phi)$ does not quite do the job since the $R \Gamma_{2}$ modules $\left(\operatorname{ind}_{H} M\right) \circ \psi$ and $\operatorname{ind}_{H}(M \circ \phi)$ are in general not equal.

We proceed as follows: First note that $\operatorname{ind}_{H}$ and the restriction res ${ }_{H}$ are adjoint functors as stated in Lemma 1.2.7, thus there are natural transformations

$$
\text { and } \quad \begin{aligned}
& \eta: \operatorname{Id}_{R \Gamma_{1}-\text { Mod }} \rightarrow \operatorname{res}_{H} \operatorname{ind}_{H} \\
& \quad \varepsilon: \operatorname{ind}_{H} \operatorname{res}_{H} \rightarrow \operatorname{Id}_{R \Gamma_{2}-\text { Mod }},
\end{aligned}
$$


the unit $(\eta)$ and counit $(\varepsilon)$ of the adjunction [Mac71, Theorem IV.1.1]. We know that these fulfill the $\Delta$-equalities, i.e., that the compositions

$$
\begin{aligned}
& \text { res } \stackrel{\eta_{\text {res }_{H}}}{\longrightarrow} \operatorname{res}_{H} \operatorname{ind}_{H} \operatorname{res}_{H} \stackrel{\operatorname{res}_{H} \varepsilon}{\longrightarrow} \operatorname{res}_{H} \\
& \text { and } \quad \operatorname{ind}_{H} \stackrel{\operatorname{ind}_{H} \eta}{\longrightarrow} \operatorname{ind}_{H} \operatorname{res}_{H} \operatorname{ind}_{H} \stackrel{\varepsilon_{\text {ind }_{H}}^{\longrightarrow}}{\longrightarrow} \operatorname{ind}_{H}
\end{aligned}
$$

are the identities. We define $H_{*}: \phi$-end $\mathrm{fff}_{R \Gamma_{1}} \rightarrow \psi-\operatorname{end}_{\mathrm{ff} R \Gamma_{2}}$ on objects of $\phi$-end $\mathrm{dff}_{\mathrm{f} \Gamma}$ to be the composition

$H_{*}:=\left(\varepsilon_{\operatorname{res}_{\psi} \operatorname{ind}_{H}}\right) \circ\left(\operatorname{ind}_{H} \operatorname{res}_{\phi}(\eta)\right) \circ \operatorname{ind}_{H}: \operatorname{Ob}\left(\phi-\operatorname{end}_{\mathrm{ff} R \Gamma_{1}}\right) \rightarrow \operatorname{Ob}\left(\psi-\operatorname{end}_{\mathrm{ff} R \Gamma_{2}}\right)$, i.e., for $\left(g: M \rightarrow \operatorname{res}_{\phi} M\right) \in \phi$-end ${\mathrm{ff} R \Gamma_{1}}_{1}$ we set

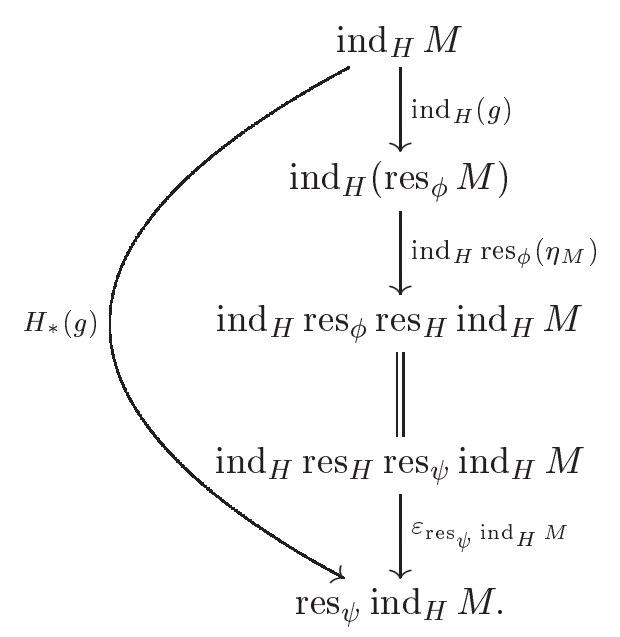

Note that $H_{*}(g)=\operatorname{ind}_{H}(g)$ if $\phi=\operatorname{Id}_{\Gamma_{1}}$ and $\psi=\operatorname{Id}_{\Gamma_{2}}$, by the $\Delta$-equalities. Furthermore, note that the unit and counit are concretely given by the maps

$$
\eta=\operatorname{id}_{M} \otimes\left(\left.H\right|_{\operatorname{Mor}(? ?, ?)}\right): \underbrace{M \otimes_{R \Gamma_{1}} R \Gamma_{1}(? ?, ?)}_{=M} \rightarrow \underbrace{M \otimes_{R \Gamma_{1}} R \Gamma_{2}(H(? ?), H(?))}_{=\operatorname{res}_{H} \operatorname{ind}_{H} M}
$$

and

$$
\begin{aligned}
& \varepsilon=\text { mult }: \overbrace{\left(K \otimes_{R \Gamma_{2}} R \Gamma_{2}(H(?), ? ?)\right) \otimes_{R \Gamma_{1}} R \Gamma_{2}(? ? ?, H(?))}^{=\operatorname{ind}_{H} \operatorname{res}_{H} K} \rightarrow K \\
& ((k, f), g) \mapsto k f g .
\end{aligned}
$$

The $R \Gamma_{2}$-module $\operatorname{ind}_{H} M$ is finite free since induction preserves this property, as stated in Remark 1.2.9. Thus $H_{*}(g)$ represents an element in $U\left(R, \Gamma_{2}, \psi\right)$. We define $H_{*}$ on morphisms

$$
\tau:(M \stackrel{g}{\rightarrow} M \circ \phi) \rightarrow\left(N \stackrel{g^{\prime}}{\rightarrow} N \circ \phi\right)
$$


by setting

$$
H_{*}(\tau):=\operatorname{ind}_{H} \tau .
$$

Since $\operatorname{ind}_{H}$ is a functor, $\operatorname{ind}_{H} \tau$ is a morphism from $\operatorname{ind}_{H} M$ to $\operatorname{ind}_{H} N$. The diagram

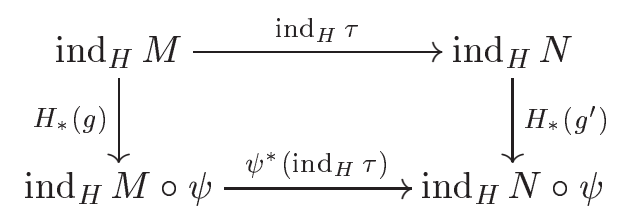

commutes since $\operatorname{ind}_{H}$ is a functor and $\eta, \varepsilon$ are natural transformations. Thus $H_{*}(\tau)=\operatorname{ind}_{H} \tau$ is a morphism in $\psi-\operatorname{end}_{\mathrm{ff} R \Gamma_{2}}$. The functorial identities $H_{*}\left(\operatorname{Id}_{\phi-\operatorname{end}_{\mathrm{ffR} \Gamma_{1}}}\right)=\operatorname{Id}_{\psi-\operatorname{end}_{\mathrm{ff} R \Gamma_{2}}}$ and $H_{*}(\tau \nu)=H_{*}(\tau) \circ H_{*}(\nu)$ are fulfilled since $\operatorname{ind}_{H}$ is a functor.

The induction functor $\operatorname{ind}_{H}$ preserves split exact sequences, so it preserves exact sequences of finite free $R \Gamma$-modules, so $H_{*}$ also preserves exact sequences since $H_{*}(\tau)=\operatorname{ind}_{H} \tau$ for all morphisms $\tau$. We have shown that $H_{*}$ is an exact functor from $\phi-\operatorname{end}_{\mathrm{ff} R \Gamma_{1}}$ to $\psi-\operatorname{end}_{\mathrm{ff} R \Gamma_{2}}$ and induces a welldefined group homomorphism $K_{0}\left(H_{*}\right): K_{0}\left(\phi\right.$-end ${\mathrm{ff} R \Gamma_{1}}_{1}) \rightarrow K_{0}\left(\psi\right.$-end ${\mathrm{ff} R \Gamma_{2}}_{2})$. We define

$$
U(R, H):=K_{0}\left(H_{*}\right): U\left(R, \Gamma_{1}, \phi\right) \rightarrow U\left(R, \Gamma_{2}, \psi\right) .
$$

Note that $H_{*}(g)$ can be seen as the composition

$$
\begin{aligned}
& \operatorname{ind}_{H} M \\
& \downarrow \operatorname{ind}_{H}(g) \\
& \operatorname{ind}_{H}\left(\operatorname{res}_{\phi} M\right)=M(?) \otimes_{R \Gamma_{1}} R \Gamma_{1}(\phi(? ?), ?) \otimes_{R \Gamma_{1}} R \Gamma_{2}(? ? ?, H(? ?)) \quad m \otimes a \otimes b \\
& \operatorname{id}_{M} \otimes H \otimes \psi \\
& M(?) \otimes_{R \Gamma_{1}} R \Gamma_{2}(H(\phi(? ?)), H(?)) \otimes_{R \Gamma_{1}} R \Gamma_{2}(\psi(? ? ?), \psi(H(? ?))) \\
& \operatorname{id}_{M} \otimes \text { mult } \\
& \operatorname{res}_{\psi} \operatorname{ind}_{H} M=M \otimes_{R \Gamma_{1}} R \Gamma_{2}(\psi(? ? ?), H(?)) \\
& m \otimes H(a) \psi(b) .
\end{aligned}
$$

This corresponds to [Lüc99, Equation 1.3]. We will frequently use this way to view $H_{*}(g)$, especially in the geometric context.

In order to have better notation at hand, we introduce the following definition.

Definition 2.2.1. Let Endo(EI-Cat) denote the category of EI-categories with endofunctors. Objects are pairs $(\Gamma, \phi)$, where $\Gamma$ is an EI-category and $\phi: \Gamma \rightarrow \Gamma$ is an endofunctor. Morphisms $F:\left(\Gamma_{1}, \phi\right) \rightarrow\left(\Gamma_{2}, \psi\right)$ are natural transformations $F: \Gamma_{1} \rightarrow \Gamma_{2}$ such that $\psi F=F \phi$. 
We want to show that $U(R,-)$ is a functor from Endo(EI-Cat) to $\mathcal{A} b$. We need the following lemma.

Lemma 2.2.2. If we have covariant functors $G:\left(\Gamma_{1}, \phi\right) \rightarrow\left(\Gamma_{2}, \psi\right)$ and $H:\left(\Gamma_{2}, \psi\right) \rightarrow\left(\Gamma_{3}, \vartheta\right)$ of EI-categories with endofunctors $\phi, \psi$ and $\vartheta$, i.e., such that $\psi G=G \phi$ and $\vartheta H=H \psi$, then $H_{*} G_{*}=(H G)_{*}$.

Proof. Let $(g: M \rightarrow M \circ \phi) \in \phi$-end $\mathrm{ff}_{R \Gamma_{1}}$. Abbreviating res by $r$ and ind by $i$ for better readability, the following diagram commutes:

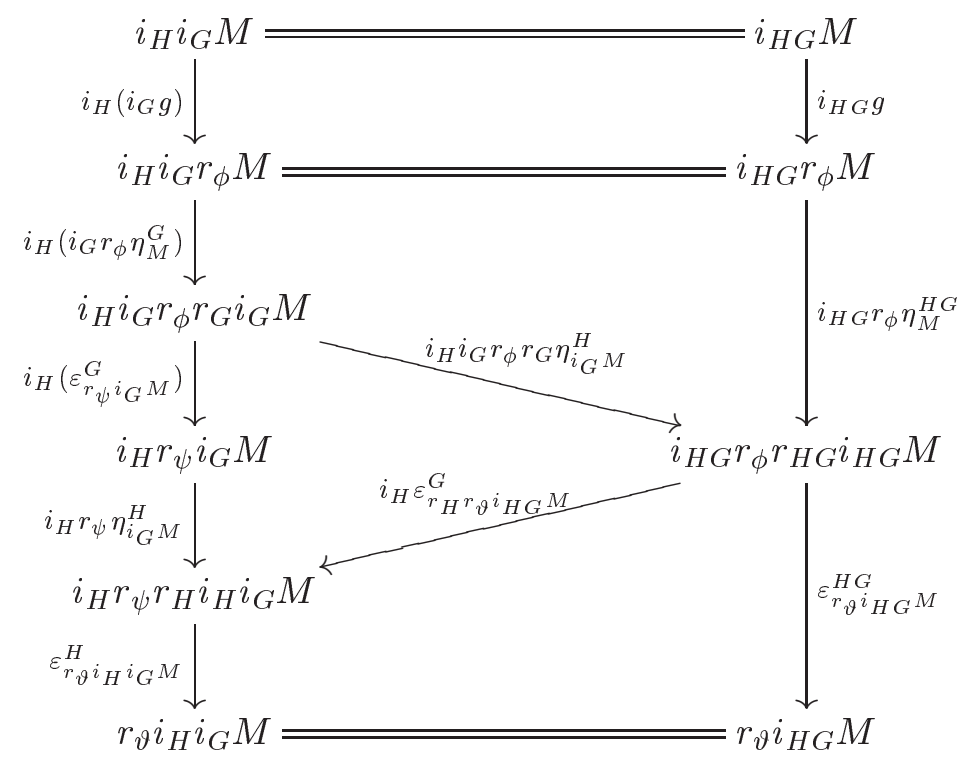

The left vertical composition is $H_{*}\left(G_{*}(g)\right)$, the right vertical composition is $(H G)_{*}(g)$, so $H_{*} G_{*}$ and $(H G)_{*}$ are equal on objects. If $\tau: g \rightarrow g^{\prime}$ is a morphism, then $H_{*} G_{*}(\tau)=\operatorname{ind}_{H} \operatorname{ind}_{G}(\tau)=\operatorname{ind}_{H G}(\tau)=(H G)_{*}(\tau)$, so they are also equal on morphisms.

It is clear by definition that $\left(\operatorname{Id}_{(\Gamma, \phi)}\right)_{*}=\operatorname{Id}_{\phi \text {-end }} \mathrm{ffR}_{\mathrm{f} \Gamma}$, so $U\left(R, \operatorname{Id}_{(\Gamma, \phi)}\right)=$ $K_{0}\left(\operatorname{Id}_{\phi-e n d} d_{f f R \Gamma}\right)=\operatorname{Id}_{U(R, \Gamma, \phi)}$. The above results imply the following proposition.

Proposition 2.2.3. The map $U(R,-)$ defined above is a functor from Endo(EI-Cat) to $\mathcal{A} b$.

Here Endo(EI-Cat) denotes the category of EI-categories with endofunctor defined in Definition 2.2.1 and $\mathcal{A} b$ denotes the category of abelian groups. But we know even more.

Proposition 2.2.4. If $H: \Gamma_{1} \stackrel{\sim}{\rightarrow} \Gamma_{2}$ is an equivalence of categories with endofunctors $\phi$ and $\psi$, i.e., such that $\psi H=H \phi$, then the natural transformation $H_{*}: \phi-\operatorname{end}_{\mathrm{ff} R \Gamma_{1}} \rightarrow \psi$-end $\mathrm{ffR}_{\mathrm{f}} \Gamma_{2}$ is also an equivalence of categories. 
Proof. We construct an inverse $H^{*}$ of $H_{*}$ and show that there are natural equivalences

$$
\varepsilon: H_{*} H^{*} \rightarrow \text { Id } \quad \text { and } \quad \eta: \text { Id } \rightarrow H^{*} H_{*} .
$$

We use the restriction functor res ${ }_{H}$ which is right adjoint to $\operatorname{ind}_{H}$ to define $H^{*}$. We define a functor

$$
\begin{aligned}
H^{*}: \psi-\operatorname{end}_{\mathrm{ff} R \Gamma_{2}} & \rightarrow \phi-\operatorname{end}_{\mathrm{ff} R \Gamma_{1}} \\
(N \stackrel{g}{\rightarrow} N \circ \psi) & \mapsto\left(\operatorname{res}_{H} N \stackrel{\operatorname{res}_{H} g}{\longrightarrow} \operatorname{res}_{H}(N \circ \psi)=\left(\operatorname{res}_{H} N\right) \circ \phi\right) \\
\tau & \mapsto \operatorname{res}_{H} \tau
\end{aligned}
$$

This is well-defined even though the natural transformation $\operatorname{res}_{H}$ in general does not respect "finitely generated" and "free". But if $H$ is an equivalence of categories it does: Let $K \in \mathrm{ff} R \Gamma_{2}$-Mod, i.e., $K \cong \bigoplus_{i=1}^{n} R \Gamma_{2}\left(?\right.$ ? , $\left.b_{i}\right)$. We want to show that $\operatorname{res}_{H} K \in \mathrm{ff} R \Gamma_{1}$-Mod. Since res ${ }_{H}$ is exact, we only need to show that $\operatorname{res}_{H} R \Gamma_{2}(? ?, b)$ is finitely generated free for $b \in \Gamma_{2}$. We know that there exists an $a \in \Gamma_{1}$ and an isomorphism $v: H(a) \stackrel{\sim}{\rightarrow} b$ since $H$ is essentially surjective, i.e., every isomorphism class of objects in $\Gamma_{2}$ is hit by $H$. Thus

$$
\operatorname{res}_{H} R \Gamma_{2}(? ?, b) \cong R \Gamma_{2}(H(?), b) \cong R \Gamma_{2}(H(?), H(a))
$$

Since $H$ is fully faithful, i.e., $H$ is bijective on morphism sets, we have $R \Gamma_{2}(H(?), H(a)) \cong R \Gamma_{1}(?, a)$, and this is finitely generated free by definition, so $\operatorname{res}_{H} R \Gamma_{2}(? ?, b) \cong R \Gamma_{1}(?, a)$ also is.

We observe that the unit and counit of the adjunction between res $H$ and $\operatorname{ind}_{H}$, the natural transformations $\eta$ and $\varepsilon$, are invertible if $H$ is an equivalence of categories: $H$ being an equivalence of categories implies that $\left.H\right|_{\operatorname{Mor}(? ?, ?)}$ is an isomorphism for all ??,? $\in \mathrm{Ob} \Gamma_{1}$. Thus

$$
\eta_{M}=\operatorname{id}_{M} \otimes H: M=M \otimes_{R \Gamma_{1}} R \Gamma_{1}(? ?, ?) \rightarrow M \otimes_{R \Gamma_{1}} R \Gamma_{2}(H(? ?), H(?))
$$

is also an isomorphism. Furthermore,

$$
\begin{aligned}
\varepsilon_{K}: \operatorname{ind}_{H}\left(\operatorname{res}_{H} K\right) & =\left(K \otimes_{R \Gamma_{2}} R \Gamma_{2}(H(? ?), ?)\right) \otimes_{R \Gamma_{2}} R \Gamma_{2}(? ? ?, H(? ?)) \\
& \rightarrow K \otimes_{R \Gamma_{2}} R \Gamma_{2}(? ? ?, ?)=K
\end{aligned}
$$

is an isomorphism because $\{H(?)\} ? \in \Gamma_{1}$ hits all isomorphism classes of $\Gamma_{2}$. This implies that every morphism in $R \Gamma_{2}$ can be factored through an object of the form $H(x)$.

We want to show that there are natural equivalences $\varepsilon: H_{*} H^{*} \rightarrow$ Id and $\eta$ : Id $\rightarrow H^{*} H_{*}$. On the modules, we define these to be the original adjunction morphisms between $\operatorname{ind}_{H}$ and $\operatorname{res}_{H}$.

1. There is a natural equivalence $\varepsilon: H_{*} H^{*} \rightarrow \mathrm{Id}$. We know that the object $H_{*} H^{*}(M \stackrel{g}{\rightarrow} M \circ \phi)$ is the left vertical composition in the following 
commutative diagram, where we abbreviate res by $r$ and ind by $i$ for better readability:

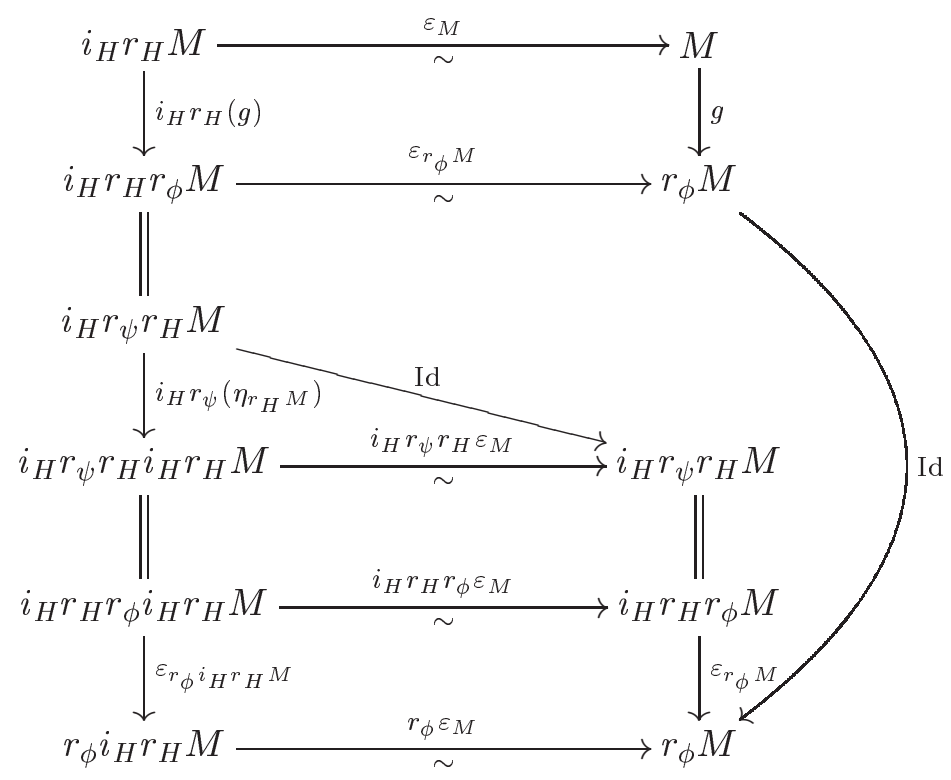

The triangle commutes because of the triangle equalities. The right vertical composition is $M \stackrel{g}{\rightarrow} M \circ \phi$, and the diagram shows that $\varepsilon_{M}$ is an isomorphism between $H_{*} H^{*}(M \stackrel{g}{\rightarrow} M \circ \phi)$ and $\operatorname{Id}(M \stackrel{g}{\rightarrow} M \circ \phi)$. We know that $\varepsilon$ is a natural transformation of functors $H_{*} H^{*} \rightarrow$ Id since on morphisms $\tau$ we have $H_{*} H^{*}(\tau)=\operatorname{ind}_{H} \operatorname{res}_{H}(\tau)$, and $\varepsilon$ is a natural transformation of functors ind $\operatorname{res}_{H} \rightarrow$ Id. So $\varepsilon$ is indeed a natural equivalence from $H_{*} H^{*}$ to Id.

2. There is a natural equivalence $\eta: \operatorname{Id~} \rightarrow H^{*} H_{*}$. We know that the object $H^{*} H_{*}(N \stackrel{g}{\rightarrow} N \circ \psi)$ is the left vertical composition in the following 
diagram, where we again abbreviate res by $r$ and ind by $i$ :

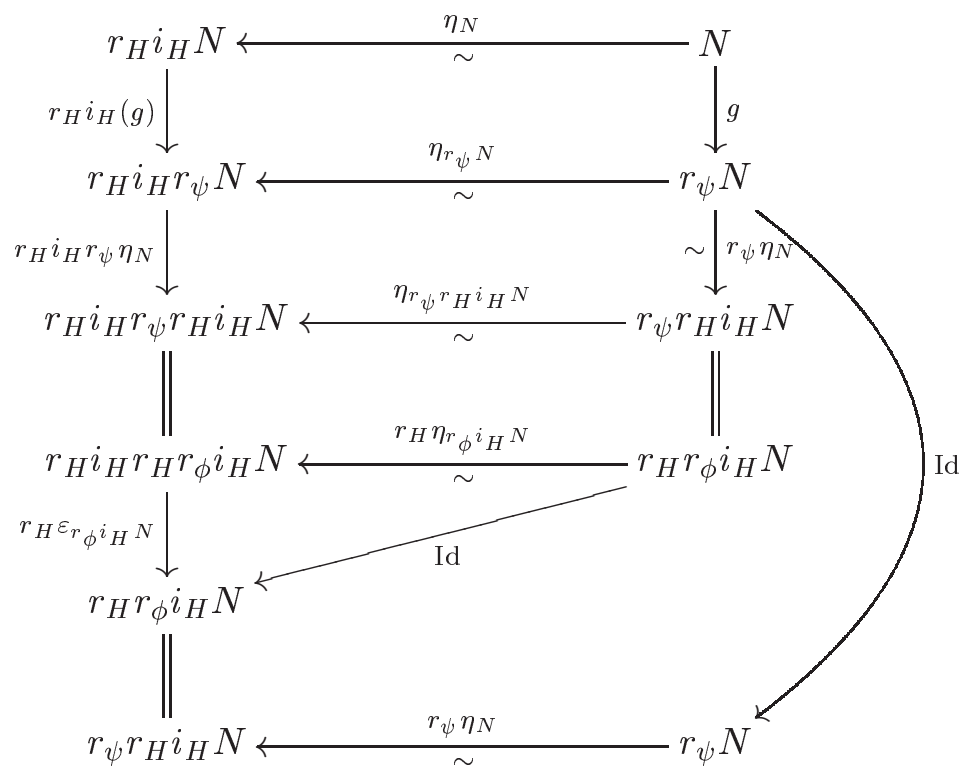

We see that the isomorphism $\eta_{N}$ sends $(N \stackrel{g}{\rightarrow} N \circ \psi)$ to $H^{*} H_{*}(N \stackrel{g}{\rightarrow}$ $N \circ \psi)$. Since on morphisms $h$ we have $H^{*} H_{*}(h)=\operatorname{res}_{H} \operatorname{ind}_{H}(h)$ and $\eta$ is a natural transformation of functors Id $\rightarrow \operatorname{res}_{H} \operatorname{ind}_{H}$, we know that $\eta$ is a natural transformation from Id to $H^{*} H_{*}$. So the natural transformation $\eta$ is a natural equivalence.

Remark 2.2.5. One could try to find a more direct proof using the fact that for an equivalence of categories $H: \Gamma_{1} \rightarrow \Gamma_{2}$ there is a functor $G: \Gamma_{2} \rightarrow \Gamma_{1}$ such that $G H$ is naturally equivalent to $\operatorname{Id}_{\Gamma_{1}}$ and $H G$ is naturally equivalent to $\operatorname{Id}_{\Gamma_{2}}$. But this is not as easy as it sounds because of the complicated definition of $H_{*}$. We decided to stick with this line of argument because it fits in better with the rest of the work and because the construction of the functor $H^{*}: \psi$-end $\mathrm{ff}_{\mathrm{f} R \Gamma_{2}} \rightarrow \phi-\operatorname{end}_{\mathrm{ff} R \Gamma_{1}}$ using the restriction functor res ${ }_{H}$ done in the proof is needed in Chapter 4, in the proof of Theorem 4.1.2.

\subsection{Additive Properties}

Now we are finally ready to analyze some of the properties of the abelian group $U(R, \Gamma, \phi)$. It is the $K_{0}$-group of a category, and one obtains a universal additive invariant for the objects of this category by taking their representatives in $U(R, \Gamma, \phi)$. We make precise what we mean by this statement. This chapter is in analogy to [Lüc99, Chapter 1].

Definition 2.3.1. Let $R$ be a commutative ring with 1 , let $\Gamma$ be an EIcategory and let $\phi: \Gamma \rightarrow \Gamma$ be an endofunctor. An additive invariant for the 
category of $\phi$-endomorphisms of finite free $R \Gamma$-chain complexes $\phi$-end $\mathrm{fff}_{\mathrm{ff}} R$-ch is a pair $(A, a)$, where $A$ is an abelian group and $a: \mathrm{Ob}\left(\phi\right.$-end $\left.\mathrm{ff}_{\mathrm{ff} \Gamma \text {-ch }}\right) \rightarrow$ $A,(g: C \rightarrow C \circ \phi) \mapsto a(g)$ is a function such that the following properties are satisfied:

\section{Additivity}

For a short exact sequence $0 \rightarrow f \rightarrow g \rightarrow h \rightarrow 0$, given by a commutative diagram with exact rows

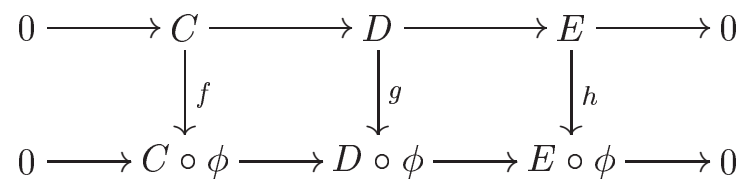

where $C, D$ and $E$ are finite free $R \Gamma$-chain complexes, we have

$$
a(f)-a(g)+a(h)=0 .
$$

2. Homotopy invariance

Let $f, g: C \rightarrow C \circ \phi$ be $R \Gamma$-chain maps of finite free $R \Gamma$-chain complexes. If $f$ and $g$ are $R \Gamma$-chain-homotopic (i.e., there is an $h: C \rightarrow$ $C \circ \phi$ of degree +1 such that $d \circ h+h \circ d=f-g)$, then $a(f)=a(g)$.

3. Elementary chain complexes

For a finite free $R \Gamma$-module $F$ and $n \geq 1$ we have

$$
a(0: \operatorname{el}(F, n) \rightarrow \operatorname{el}(F, n) \circ \phi)=0,
$$

where el $(F, n)$ denotes the elementary chain complex which is concentrated in dimensions $n$ and $n-1$ and has $n$-th differential Id: $F \rightarrow F$.

Definition 2.3.2. An additive invariant $(U, u)$ for the category $\phi$-end $\mathrm{ff}_{\mathrm{ff}} R$-ch is called universal if and only if for all additive invariants $(A, a)$ for the category $\phi$-end $\mathrm{fff}_{\mathrm{f} \Gamma \text {-ch }}$ there is exactly one group homomorphism $\xi: U \rightarrow A$ such that $\xi(u(g))=a(g)$ for all $g \in \phi$-end ff $R \Gamma$-ch $_{\text {. }}$

In Section 2.1, we defined the abelian group $U(R, \Gamma, \phi)$ and the function

$$
\begin{aligned}
\phi \text { end }_{\mathrm{ff} R \Gamma-\mathrm{ch}} & \rightarrow U(R, \Gamma, \phi) \\
(g: C \rightarrow C \circ \phi) & \mapsto \sum_{n \in \mathbb{Z}}(-1)^{n}\left[g_{n}\right] .
\end{aligned}
$$

We now show that this pair is the universal additive invariant for the category $\phi$-end $\mathrm{d}_{\mathrm{ff} R \Gamma-\mathrm{ch}}$.

Theorem 2.3.3. The pair $(U(R, \Gamma, \phi), u)$ is the universal additive invariant for $\phi$-end $\mathrm{ff}_{\mathrm{f} R \Gamma \text {-ch }}$. 
Proof. Property 1, additivity, is clear by definition of $K_{0}$, thus of $U(R, \Gamma, \phi)$. Property 3 is also clear since

$$
\begin{aligned}
& u(0: \operatorname{el}(F, n) \rightarrow \operatorname{el}(F, n) \circ \phi) \\
& \quad=(-1)^{n}\left(\left[0_{n}: F \rightarrow F \circ \phi\right]-\left[0_{n+1}: F \rightarrow F \circ \phi\right]\right) \\
& \quad=0 \in U(R, \Gamma, \phi) .
\end{aligned}
$$

The proof of property 2 uses the mapping cone and the suspension of $C$. Let $h: C \rightarrow C \circ \phi$ be an $R \Gamma$-chain homotopy from $f$ to $g$. Let $\Sigma C$ be the suspension of $C$, the $R \Gamma$-chain complex with differential

$$
(\Sigma C)_{n}=C_{n-1} \stackrel{-d_{n-1}}{\longrightarrow}(\Sigma C)_{n-1}=C_{n-2},
$$

and cone $(C):=$ cone $\left(\operatorname{id}_{C}\right)$ the mapping cone of $C[\operatorname{Lüc89}, 11.14]$, i.e., the $R \Gamma$-chain complex with differential

$$
\operatorname{cone}(C)_{n}=C_{n-1} \oplus C_{n} \stackrel{\left(\begin{array}{cc}
-d_{n-1} & 0 \\
\text { id } & d_{n}
\end{array}\right)}{\longrightarrow} C_{n-2} \oplus C_{n-1}=\operatorname{cone}(C)_{n-1}
$$

We obtain an $R \Gamma$-chain map $k: \operatorname{cone}(C) \rightarrow \operatorname{cone}(C) \circ \phi$ by setting

$$
k_{n}:=\left(\begin{array}{cc}
g_{n-1} & 0 \\
h_{n-1} & f_{n}
\end{array}\right): C_{n-1} \oplus C_{n} \rightarrow\left(C_{n-1} \oplus C_{n}\right) \circ \phi
$$

such that we have a commutative diagram with exact rows

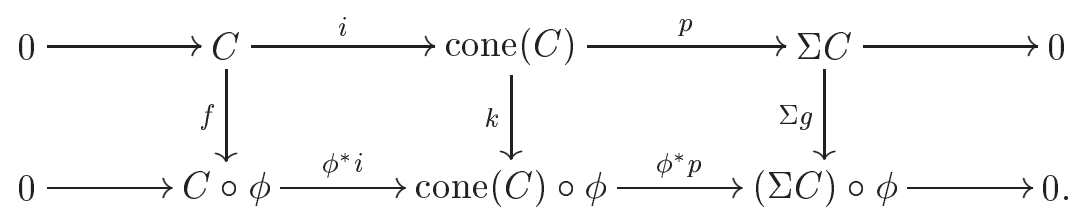

We conclude from additivity that

$$
u(f)-u(g)=u(f)+u(\Sigma g)=u(k) .
$$

Note that cone $(C)$ is a contractible $R \Gamma$-chain complex [Lüc89, 11.14]. Hence the proof is finished if we can show that for contractible $C$ and for any $f: C \rightarrow C \circ \phi$ we have

$$
u(f: C \rightarrow C \circ \phi)=0 \in U(R, \Gamma, \phi) .
$$

We do this by induction on the dimension $d$ of $C$. If $d \leq 2$, existence of a chain contraction is equivalent to exactness, and the claim follows by definition of $u$ and $U(R, \Gamma, \phi)$.

Induction step $d(\geq 2) \rightarrow d+1$ : Let $D$ be the $R \Gamma$-subchain complex of $C$ given by $D_{d+1}=C_{d+1}, D_{d}=\operatorname{ker}\left(c_{d}\right)$ and $D_{p}=0$ for $p \neq d, d+1$. Let $E:=\operatorname{coker}(D \stackrel{i}{\rightarrow} C)$. Since $C$ is a finite free contractible $R \Gamma$-chain 
complex, we can assume without loss of generality that $D$ and $E$ are finite free contractible $R \Gamma$-chain complexes, otherwise add to $C$ the elementary chain complex el $\left(C_{d+1}, d\right)$. The $R \Gamma$-chain map $f$ induces $R \Gamma$-chain maps $g: D \rightarrow \phi^{*} D$ and $h: E \rightarrow \phi^{*} E$. Now from additivity one gets

$$
u(g)-u(f)+u(h)=0 .
$$

By the induction hypothesis, $u(g)=0=u(h)$. Thus $u(f)=0$.

The universal property of $(U(R, \Gamma, \phi), u)$ remains to be shown. Let $(A, a)$ be another additive invariant. Define $\xi: U(R, \Gamma, \phi) \rightarrow A$ by setting $\xi([g]):=$ $a(g)$, where $g: M \rightarrow M \circ \phi$ is a $\phi$-endomorphism of a finite free $R \Gamma$-module (which can be viewed as a chain complex concentrated in degree 0). By additivity of $(A, a)$, the fact that $g \cong h$ implies $a(g)=a(h)$, and exactness of $0 \rightarrow g \rightarrow h \rightarrow k \rightarrow 0$ implies $a(g)-a(h)+a(k)=0$, thus $\xi$ is well-defined. If $f: C \rightarrow C \circ \phi$ is a $\phi$-endomorphism of a finite free chain complex $C$, then $\xi \circ u(f)=\sum_{n \in \mathbb{Z}}(-1)^{n} \xi\left(\left[f_{n}\right]\right)=\sum_{n \in \mathbb{Z}}(-1)^{n} a\left(f_{n}\right)$. We need to show that this equals $a(f)$. This is again proved by induction on the dimension $d$ of $C$. If $d=0$, then the claim is trivial. Let $D$ be the $R \Gamma$-chain complex concentrated in dimension $d+1$ satisfying $D_{d+1}=C_{d+1}$. Let $\left.C\right|_{d}$ be the chain complex obtained by truncating $C$, i.e., equal to $C$ in dimensions $\leq d$ and 0 else. Let $g:\left.\left.C\right|_{d} \rightarrow C\right|_{d} \circ \phi$ and $h: D \rightarrow D \circ \phi$ be the $R \Gamma$-chain maps induced by $f$. From additivity and the exactness of $\left.0 \rightarrow C\right|_{d} \rightarrow C \rightarrow D \rightarrow 0$ we conclude $a(g)-a(f)+a(h)=0$. There is an exact sequence

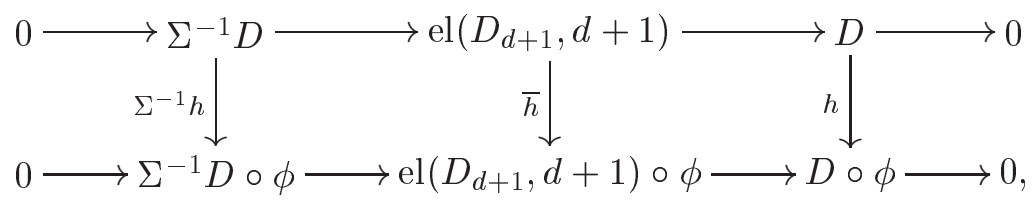

with $\bar{h}$ given by

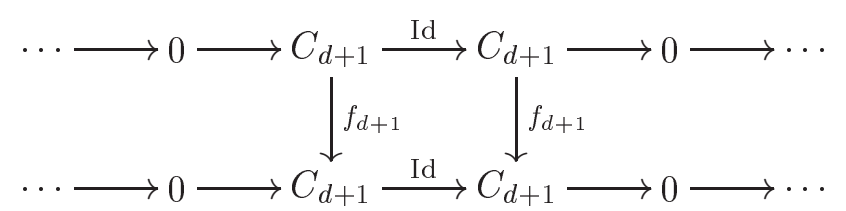

An $R \Gamma$-chain contraction of this complex is given by $f_{d+1}$ in degree $d$ and by 0 in the other degrees. By additivity we have $a\left(\Sigma^{-1} h\right)-a(\bar{h})+a(h)=0$ and by properties 2 and 3 we have $a(\bar{h})=a\left(0: \operatorname{el}\left(C_{d+1}, d+1\right) \rightarrow \operatorname{el}\left(C_{d+1}, d+1\right) \circ\right.$ $\phi)=0$, thus $a(h)=-a\left(\Sigma^{-1} h\right)$. By the induction hypothesis, $-a\left(\Sigma^{-1} h\right)=$ $-(-1)^{d} a\left(f_{d+1}\right)$ and $a(g)=\sum_{n=0}^{d}(-1)^{n} a\left(f_{n}\right)$, thus $a(f)=\sum_{n=0}^{d+1}(-1)^{n} a\left(f_{n}\right)$ as was to be shown.

We have shown the existence of a map $\xi: U(R, \Gamma, \phi) \rightarrow A$ with the desired properties. Any other such map $\xi^{\prime}$ has to fulfill $\xi^{\prime}(u(g))=a(g)$ for 0 -dimensional chain complexes, thus $\xi^{\prime}([g])=a(g)$, and so $\xi=\xi^{\prime}$ since they agree on generators. Uniqueness is proved, and we are finished. 
From the proof of the theorem, we can conclude that also the following lemma is true:

Lemma 2.3.4. Let

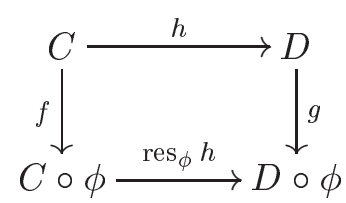

be a commutative diagram of finite free $R \Gamma$-chain complexes. If $h$ is a chain homotopy equivalence, then $u(f)=u(g)$ in $U(R, \Gamma, \phi)$.

Proof. We have a commutative diagram with exact rows

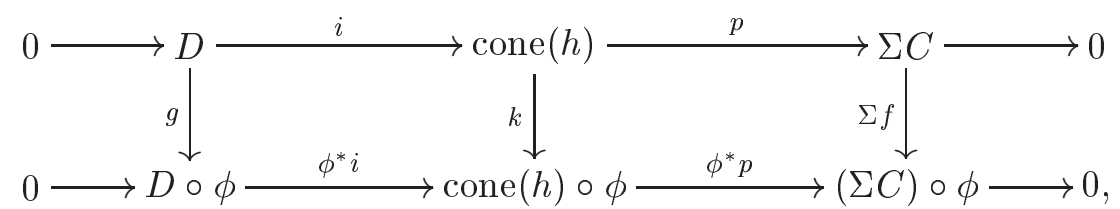

where cone $(h)$ is the chain complex with differential

$$
\operatorname{cone}(h)_{n}=C_{n-1} \oplus D_{n} \stackrel{\left(\begin{array}{cc}
-c_{n-1} & 0 \\
h_{n-1} & d_{n}
\end{array}\right)}{\longrightarrow} C_{n-2} \oplus D_{n-1}
$$

and where $k_{n}=\left(\begin{array}{cc}-f_{n-1} & 0 \\ h_{n-1} & g_{n}\end{array}\right)$. We conclude from additivity that $u(g)-$ $u(f)=u(k)$. We know that cone $(h)$ is contractible if and only if $h$ is a chain homotopy equivalence $[\mathrm{Lüc} 89,11.5]$. In the proof of the theorem we have just shown that the contractibility of cone $(h)$ implies that $u(k)=0 \in U(R, \Gamma, \phi)$, so $u(g)=u(f)$.

If $R$ is a commutative ring which is not the zero ring, $R$ has the property that $R^{n} \cong R^{m}$ implies $n=m$, i.e., a finite free $R$-module has a welldefined rank. One sees analogously to [Lüc99, Chapter 1] that the invariant $(U(R, \Gamma, \phi), u(R, \Gamma, \phi))$ incorporates the Euler characteristic. Recall that the Euler characteristic of a finite free $R$-chain complex $C$ is defined by

$$
\chi(C):=\sum_{n \geq 0}(-1)^{n} \operatorname{rk}_{R}\left(C_{n}\right) \in \mathbb{Z} .
$$

An analogous notion for finite free $R \Gamma$-chain complexes can be defined. Let $U(\Gamma)$ be the free abelian group generated by the set $\operatorname{Is}(\Gamma)$ of isomorphism classes of objects [Lüc89, Definition 10.9],

$$
U(\Gamma):=\bigoplus_{\operatorname{Is} \Gamma} \mathbb{Z}
$$


Let ff $R \Gamma$ denote the category of finite free $R \Gamma$-modules, and let $K_{0}^{f}(R \Gamma):=$ $K_{0}($ ff $R \Gamma)$ [Lüc89, Definition 10.7]. Then we have natural transformations rk and $j$ between the covariant functors $K_{0}^{f}$ and $U$ from EI-Cat to $\mathcal{A} b$,

$$
\begin{array}{rll} 
& \text { rk: } \quad K_{0}^{f} \rightarrow U, \quad[M] \mapsto\left(\operatorname{rk}_{R}\left(S_{x} M \otimes_{R[x]} R\right)\right)_{\bar{x} \in \operatorname{Is} \Gamma} \\
\text { and } \quad j: & U \rightarrow K_{0}^{f}, \quad\left(n_{\bar{x}}\right)_{\bar{x} \in \operatorname{Is} \Gamma} \mapsto \sum_{\bar{x} \in \operatorname{Is} \Gamma} n_{\bar{x}} \cdot[R \operatorname{Mor}(?, x)] .
\end{array}
$$

The natural transformations rk and $j$ are natural equivalences inverse to each other [Lüc89, Propositions 10.42 and 10.44]. For all EI-categories $\Gamma$ we have inverse isomorphisms of abelian groups

$$
K_{0}^{f}(R \Gamma) \underset{j_{R \Gamma}}{\stackrel{\mathrm{rk} k_{R \Gamma}}{\rightleftarrows}} U(\Gamma) .
$$

It is clear that we have a forgetful functor given by forget: $\phi$-end $\mathrm{f}_{\mathrm{ff} R \Gamma} \rightarrow$ ff $R \Gamma,[g: M \rightarrow M \circ \phi] \mapsto[M]$ which only takes the modules and forgets the morphisms. In $K_{0}$, this functor induces a group homomorphism $p_{(\Gamma, \phi)}:=K_{0}$ (forget) $: U(R, \Gamma, \phi) \rightarrow K_{0}^{f}(R \Gamma)$. Since in both categories, $\phi-\operatorname{end}_{\mathrm{ff} R \Gamma}$ and $\mathrm{ff} R \Gamma$, the functoriality in $\Gamma$ is given on the modules $M$ by the induction functor, this functoriality is respected by the forgetful functor. So the functor $p: U(R,-) \rightarrow K_{0}^{f}(R-)$ is a natural transformation of covariant functors from Endo(EI-Cat) to $\mathcal{A} b$. Composing it with the functor $\mathrm{rk}: K_{0}^{f}(R-) \rightarrow U(-)$ gives a natural transformation of covariant functors

$$
\text { rkop:U(R,-) } \rightarrow U(-)
$$

from Endo(EI-Cat) to $\mathcal{A} b$.

This leads to a generalization of the Euler characteristic. For a finite free $R \Gamma$-chain complex $C$ we define

$$
\chi(C):=\operatorname{rk}_{R \Gamma}([C])=\sum_{n \in \mathbb{Z}}(-1)^{n} \operatorname{rk}\left(\left[C_{n}\right]\right) \in U(\Gamma)
$$

to be the Euler characteristic of $C$. We can combine this with the forgetful functor and see that the Euler characteristic of $C$ can be read off from $u(f: C \rightarrow C \circ \phi)$ for any $\phi$-endomorphism $f$ of $C$ :

$$
\chi(C)=\operatorname{rk}([C])=\operatorname{rk} \circ p([f: C \rightarrow C \circ \phi])=\operatorname{rk} \circ p(u(f)) .
$$

One can always assign the $\phi$-endomorphism 0: $C \rightarrow C \circ \phi$ to an $R \Gamma$-chain complex $C$, we have a natural transformation $i$ given by $i: K_{0}^{f}(R \Gamma) \rightarrow$ $U(R, \Gamma, \phi),[M] \mapsto[0: M \rightarrow M \circ \phi]$. This is a splitting of the forgetful functor. Also, one can always assign the identity functor to a category $\Gamma$ if there is no other one around, this gives an inclusion EI-Cat $\rightarrow$ Endo(EI-Cat), so 
$U(R, \Gamma, \phi)$ is a generalization of $K_{0}^{f}(R \Gamma)$. One obtains natural transformations $s:=i \circ j$ and $\operatorname{dim}:=$ rk $\circ p$, given by

$s:=i \circ j$

$U(\Gamma) \rightarrow U(R, \Gamma, \phi)$

$\left(m_{\bar{x}}\right)_{\bar{x} \in \operatorname{Is}(\Gamma)} \mapsto \sum_{\bar{x} \in \operatorname{Is}(\Gamma)} \operatorname{sign}\left(m_{\bar{x}}\right) \cdot\left[0:\left|m_{\bar{x}}\right| \cdot R \Gamma(?, x) \rightarrow\left|m_{\bar{x}}\right| \cdot R \Gamma(\phi(?), x)\right]$

$\operatorname{dim}:=\operatorname{rk} \circ p:$

$$
U(R, \Gamma, \phi) \rightarrow U(\Gamma)
$$

$[g: M \rightarrow M \circ \phi] \mapsto \operatorname{rk}_{R \Gamma} M=\left(\operatorname{rk}_{R}\left(S_{x} M \otimes_{R[x]} R\right)\right)_{\bar{x} \in \mathrm{Is}(\Gamma)}$.

We have

$$
\operatorname{dim} \circ s=(\operatorname{rk} \circ p) \circ(i \circ j)=\operatorname{Id}_{U(\Gamma)}
$$

because $p \circ i=\operatorname{Id}_{K_{0}^{f}(\Gamma)}$ and $\mathrm{rk} \circ j=\operatorname{Id}_{U(\Gamma)}[$ Lüc89, 10.42]. We also know that $j \circ \mathrm{rk}=\operatorname{Id}_{K_{0}^{f}(R-)}$, so the composition $i \circ p$ is $i \circ p=i \circ j \circ \mathrm{rk} \circ p=s \circ \operatorname{dim}$, given by

$$
\begin{aligned}
s \circ \operatorname{dim}: U(R, \Gamma, \phi) & \rightarrow U(R, \Gamma, \phi) \\
{[g: M \rightarrow M \circ \phi] } & \mapsto[0: M \rightarrow M \circ \phi] .
\end{aligned}
$$

We obtain the following lemma, which is analogous to [Lüc99, Lemma 1.6].

Lemma 2.3.5. Let $v: C \rightarrow D$ and $f: C \rightarrow C \circ \phi$ be $R \Gamma$-chain maps, where $C$ and $D$ are finite free $R \Gamma$-chain complexes. Then

$$
u(f \circ v)+s(\chi(D))=u\left(\phi^{*} v \circ f\right)+s(\chi(C)) .
$$

Proof. We consider the commutative diagram

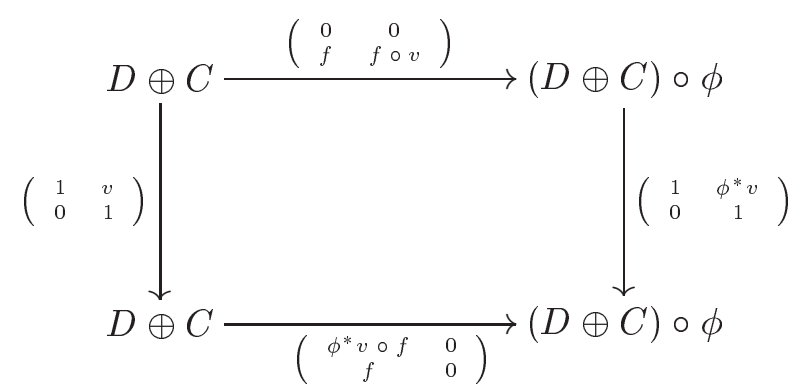

Since the vertical arrows are isomorphisms, we get from additivity

$$
u\left(\left(\begin{array}{cc}
0 & 0 \\
f & f \circ v
\end{array}\right)\right)=u\left(\left(\begin{array}{cc}
\phi^{*} v \circ f & 0 \\
f & 0
\end{array}\right)\right) .
$$

Again from additivity we derive

$$
u(f \circ v)+u\left(0: D \rightarrow \phi^{*} D\right)=u\left(\left(\begin{array}{cc}
0 & 0 \\
f & f \circ v
\end{array}\right)\right)
$$

and

$$
u\left(\phi^{*} v \circ f\right)+u\left(0: C \rightarrow \phi^{*} C\right)=u\left(\left(\begin{array}{ccc}
\phi^{*} v \circ f & 0 \\
f & 0
\end{array}\right)\right) .
$$




\section{Chapter 3}

\section{The Geometric Approach}

In this chapter, we discuss the geometric setting. We explain how the algebraic definitions and results of Chapter 2 can be applied to the geometric situation. We specify the geometric version of the abelian group $U(R, \Gamma, \phi)$, an abelian group called $U_{G}^{R}(X, f)$. For the ring $R=\mathbb{Z}$, the group $U_{G}^{\mathbb{Z}}(X, f)$ is the target group of the universal functorial equivariant Lefschetz invariant which we are constructing.

We define an element $u_{G}^{R}(X, f) \in U_{G}^{R}(X, f)$, which for the $\operatorname{ring} R=\mathbb{Z}$ will turn out to be the universal functorial equivariant Lefschetz invariant.

We state which properties we expect of a functorial equivariant Lefschetz invariant, for example functoriality in $(X, f)$ and an induction structure in $G$. We proceed to prove these properties for the pair $\left(U_{G}^{R}(X, f), u_{G}^{R}(X, f)\right)$. The fact that $\left(U_{G}^{\mathbb{Z}}(X, f), u_{G}^{\mathbb{Z}}(X, f)\right)$ is the universal functorial equivariant Lefschetz invariant will be shown in Chapter 4.

\subsection{The Definition of the Geometric Invariant}

Let $G$ be a discrete group. Let $G-\mathcal{C} W_{\mathrm{fp}}$ be the category of finite proper $G$-CW-complexes, see Definition 1.1.4. Let $\operatorname{End}\left(G-\mathcal{C} W_{\mathrm{fp}}\right)$ be the category of $G$-endomorphisms of finite proper $G$-CW-complexes.

Given a finite proper $G$-CW-complex $X$ and a $G$-equivariant cellular endomorphism $f: X \rightarrow X$, we want to introduce an abelian group $U_{G}^{R}(X, f)$ and an invariant $u_{G}^{R}(X, f) \in U_{G}^{R}(X, f)$. (Note that we can drop the assumption that $f: X \rightarrow X$ is cellular at the end of this chapter because of homotopy invariance of our invariants.)

In the non-equivariant case [Lüc99], the construction uses the universal covering space $\widetilde{X}$ of the CW-complex $X$. Choosing a basepoint $x$, the fundamental group $\pi_{1}(X, x)$ acts on $\widetilde{X}$. One can lift $f: X \rightarrow X$ to a $\phi$-equivariant morphism $\tilde{f}: \widetilde{X} \rightarrow \widetilde{X}$, where $\phi:=c_{w} \circ \pi_{1}(f, x): \pi_{1}(X, x) \rightarrow \pi_{1}(X, x)$ for a chosen path $w$ from $f(x)$ to $x$. We generalize this non-equivariant construction to $G$-CW-complexes. 
The natural generalization of the fundamental group is the fundamental category $\Pi(G, X)$ (which is actually a generalization of the fundamental groupoid). Recall from Definition 1.2.2 that objects in the fundamental category $\Pi(G, X)$ are $G$-maps $x: G / H \rightarrow X$ for $H \leq G$, morphisms are pairs $(\sigma,[w]): x(H) \rightarrow y(K)$ consisting of a $G$-map $\sigma: G / H \rightarrow G / K$ and a homotopy class $[w]$ relative $G / H \times \partial I$ of $G$-maps $w: G / H \times I \rightarrow X$ with $w_{1}=$ $x$ and $w_{0}=y \circ \sigma$ [Lüc89, Definition 8.15]. This construction is functorial in $X$ (by composition of maps), in particular the $G$-equivariant endomorphism $f: X \rightarrow X$ induces an endofunctor $\phi:=\Pi(G, f): \Pi(G, X) \rightarrow \Pi(G, X)$.

An advantage of using the fundamental category is that there are no choices involved. We need not worry about them until Chapter 6 , where we try to get back from groupoids to groups.

There is the contravariant universal covering functor $\widetilde{X}: \Pi(G, X) \rightarrow \mathcal{T}$ op at hand [Lüc89, Definition 8.22], generalizing perfectly well the universal covering space $\widetilde{X}$ of a topological space $X$. It is the contravariant functor $\widetilde{X}: \Pi(G, X) \rightarrow \mathcal{T}$ op mapping $x \in \Pi(G, X)$ to $\widetilde{X^{H}(x)}$ and $(\sigma,[w]) \in$ $\operatorname{Mor}(x(H), y(K))$ to the map $\widehat{X^{K}(y)} \rightarrow \widehat{X^{H}(x)}$ induced by composition of morphisms. Here $X^{H}(x)$ denotes the connected component of the fixed point set $X^{H}$ containing the point $x(1 H)$, for an object $x: G / H \rightarrow X$ of $\Pi(G, X)$. If $X$ is a proper $G$-CW-complex, $\widetilde{X}$ lands in the category $\mathcal{C} W$ of CW-complexes with cellular maps as morphisms (here even: simply connected) [Lüc89, Proposition 8.33]. Composing the universal covering functor $\widetilde{X}$ with the cellular chain complex functor $C^{c}: \mathcal{C} W \rightarrow R$-C $h$ landing in the category $R$-C $h$ of $R$-chain complexes one obtains the cellular $R \Pi(G, X)$ chain complex $C^{c}(X ; R)$ as a contravariant functor [Lüc89, Definition 8.37]

$$
C^{c} \circ \widetilde{X}: \Pi(G, X) \stackrel{\widetilde{X}}{\rightarrow} \mathcal{C} W \stackrel{C^{c}}{\rightarrow} R-\mathcal{C} h .
$$

We land in the category of finite $R$-chain complexes if $X$ is a finite proper $G$-CW-complex. The functor $C^{c} \circ \widetilde{X}$ is even a finite free $R \Pi(G, X)$-chain complex [Lüc89, 9.18].

The map $f: X \rightarrow X$ induces a $\phi$-endomorphism $C^{c}(\widetilde{f})$ of the cellular $R \Pi(G, X)$-chain complex $C^{c} \circ \widetilde{X}$. We first define a $\phi$-endomorphism $\widetilde{f}$ of $\widetilde{X}$ by setting for $(x: G / H \rightarrow X) \in \Pi(G, X)$

$$
\widetilde{f}(x):=\widetilde{\left.f\right|_{X^{H}(x)}}: \widetilde{X^{H}(x)}=\widetilde{X}(x) \rightarrow \widetilde{X} \circ \phi(x)=\widetilde{X^{H}(f \circ x)} .
$$

The map $\left.f\right|_{X^{H}(x)}: X^{H}(x) \rightarrow X^{H}(f(x))$ is well-defined. It induces a unique map $\widetilde{\left.f\right|_{X^{H}(x)}}$ of the universal covering spaces if we require the canonical basepoint $\left[\mathrm{id}_{x}\right]$ of $\widehat{X^{H}(x)}$ to be sent to the canonical basepoint $\left[\operatorname{id}_{f(x)}\right]$ of $\widetilde{H^{H}(f \circ x)}$ since both of these spaces are simply connected. 
The map $\widetilde{f}$ is a natural transformation from $\widetilde{X}$ to $\widetilde{X} \circ \phi$ since for all $((\sigma, w): x(H) \rightarrow y(K)) \in \operatorname{Mor}_{\Pi(G, X)}(x, y)$ the following diagram commutes:

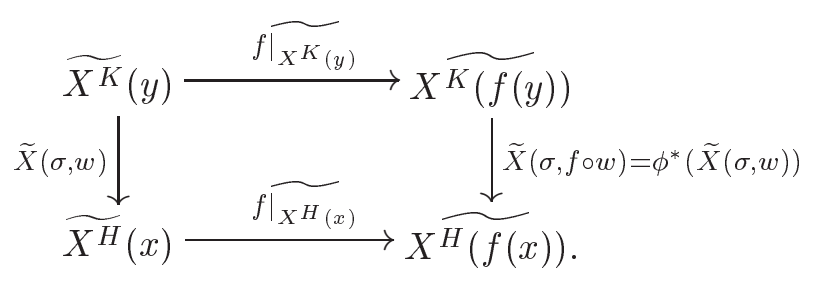

In principle this is clear by definition, but we can also calculate it explicitly: The definition of the universal covering space [Lüc89, 8.21]

$$
\widetilde{X}^{K}(y)=\left\{\left(\sigma_{y}, w_{y}\right): y \rightarrow z \mid \sigma_{y}=\operatorname{id}_{G / K}\right\}
$$

implies that for a point in $\widetilde{X}^{K}(y)$ given by $\left(\left(\operatorname{id}_{G / K}, w_{y}\right): y \rightarrow z\right)$ we have

$$
\begin{aligned}
& \widetilde{\left.f\right|_{X^{H}(x)}} \circ \tilde{X}(\sigma, w)\left(\left(\operatorname{id}_{G / K}, w_{y}\right): y \rightarrow z\right) \\
& =\widetilde{\left.f\right|_{X^{H}(x)}}\left(\left(\operatorname{id}_{G / H},\left(w_{y} \circ \sigma\right) w\right): x \rightarrow z \circ \sigma\right) \\
& =\left(\operatorname{id}_{G / H}, f \circ\left(\left(w_{y} \circ \sigma\right) w\right)\right): f(x) \rightarrow f(z \circ \sigma)
\end{aligned}
$$

and that

$$
\begin{aligned}
\widetilde{X} & (\sigma, f \circ w) \circ \widetilde{\left.f\right|_{X^{K}(y)}}\left(\left(\operatorname{id}_{G / K}, w_{y}\right): y \rightarrow z\right) \\
& =\widetilde{X}(\sigma, f \circ w)\left(\left(\operatorname{id}_{G / K}, f \circ w_{y}\right): f(y) \rightarrow f(z)\right) \\
& =\left(\operatorname{id}_{G / H},\left(\left(f \circ w_{y}\right) \circ \sigma\right)(f \circ w)\right): f(x) \rightarrow f(z) \circ \sigma \\
& =\left(\operatorname{id}_{G / H}, f \circ\left(\left(w_{y} \circ \sigma\right) w\right)\right): f(x) \rightarrow f(z \circ \sigma) .
\end{aligned}
$$

So the two ways around the diagram are equal.

If $f: X \rightarrow X$ is cellular, then $\left.f\right|_{X^{H}(x)}$ is cellular for all $x \in \Pi(G, X)$, and thus also all $\widetilde{\left.f\right|_{X^{H}(x)}}$ are cellular. We apply the cellular chain complex functor $C^{c}$ to this collection of maps. All the $\widetilde{f}(x)$ are morphisms in the category of (simply connected) CW-complexes, thus all $C^{c}(\widetilde{f}(x))$ are morphisms in the category of $R$-chain complexes:

$$
C^{c}(\widetilde{f}(x)): C^{c}(\widetilde{X}(x)) \rightarrow C^{c}(\widetilde{X} \circ \phi(x))=\left(C^{c} \circ \widetilde{X}\right)(\phi(x)) .
$$

For all morphisms $(\sigma, w): x(H) \rightarrow y(K)$ in $\Pi(G, X)$ the diagram

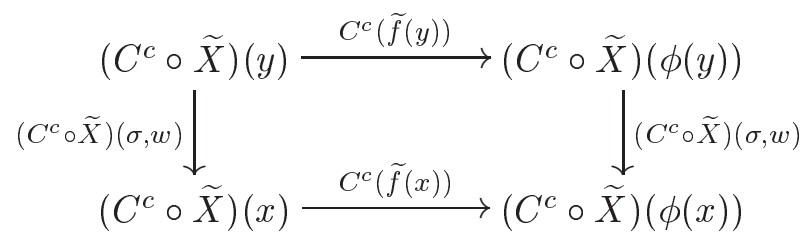


commutes since we have shown such a commutativity for $\widetilde{X}$ and $C^{c}$ is a functor:

$$
\begin{aligned}
C^{c}(\widetilde{f}(x)) \circ\left(C^{c} \circ \widetilde{X}\right)(\sigma, w) & =C^{c}(\widetilde{f}(x) \circ \widetilde{X}(\sigma, w)) \\
& =C^{c}(\widetilde{X}(\sigma, f \circ w) \circ \widetilde{f}(y)) \\
& =C^{c}(\widetilde{X}(\sigma, f \circ w)) \circ C^{c}(\widetilde{f}(y)) \\
& =\phi^{*} C^{c}(\widetilde{X}(\sigma, w)) \circ C^{c}(\widetilde{f}(y)) .
\end{aligned}
$$

So the collection of the $C^{c}(\widetilde{f}(x))$ for all $x \in \Pi(G, X)$ is a natural transformation of functors $C^{c}(\widetilde{f}): C^{c} \circ \widetilde{X} \rightarrow C^{c} \circ \widetilde{X} \circ \phi$. It defines a $\phi$-endomorphism of the cellular $R \Pi(G, X)$-chain complex $C^{c} \circ \widetilde{X}: \Pi(G, X) \rightarrow R-\mathcal{C} h$.

We are finally ready for the definition lying at the heart of all further study.

Definition 3.1.1. Let $R$ be a commutative ring with 1 , let $G$ be a discrete group, let $X$ be a finite proper $G$-CW-complex and let $f: X \rightarrow X$ be a $G$-equivariant cellular endomorphism. We set

$$
U_{G}^{R}(X, f):=U(R, \Pi(G, X), \phi)
$$

We define the element $u_{G}^{R}(X, f) \in U_{G}^{R}(X, f)$ to be

$$
u\left(C^{c}(\widetilde{f})\right) \in U(R, \Pi(G, X), \phi)=U_{G}^{R}(X, f) .
$$

Here $(U(R, \Pi(G, X), \phi), u)$ is the universal additive invariant for the category $\phi$-end $\mathrm{ff}_{\mathrm{f} R \Pi(G, X) \text {-ch introduced in Chapter } 2 .}$

\subsection{An Illustrative Example}

It is time to illustrate the above discussion with a concrete example. We have one in mind which is simple enough to be treated explicitly, while still providing an illustration of the basic elements coming up, namely $G=\mathbb{Z} / 2$, $X=S^{1}$. Let $G=\mathbb{Z} / 2=\{1, \tau\}$ act on $S^{1}=\{z \in \mathbb{C}|| z \mid=1\}$ by reflection on the real axis: $\tau\left(e^{i \varphi}\right)=e^{-i \varphi}$. For $d \in \mathbb{N}$ we define the $\mathbb{Z} / 2$-equivariant morphism

$$
\begin{aligned}
f: S^{1} & \rightarrow S^{1} \\
z & \mapsto z^{d} .
\end{aligned}
$$




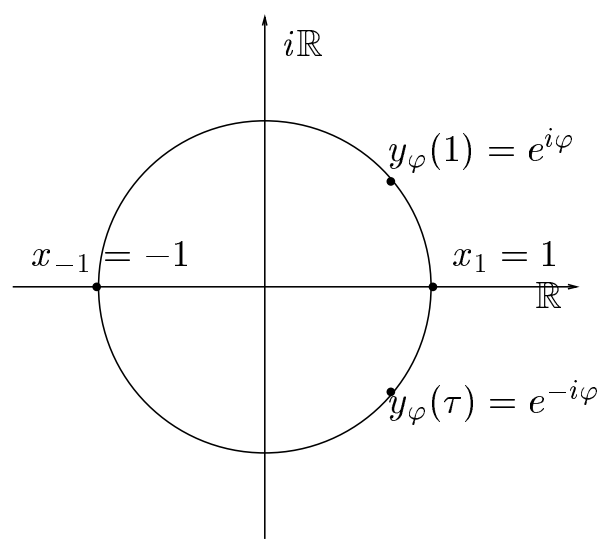

The fundamental category $\Pi\left(\mathbb{Z} / 2, S^{1}\right)$ is given by

- Objects:

$$
\begin{aligned}
x_{1}: G / G & \rightarrow S^{1}, \quad 1 \mapsto 1 \\
x_{-1}: G / G & \rightarrow S^{1}, \quad 1 \mapsto-1 \\
y_{\varphi}: G /\{1\} & \rightarrow S^{1}, \quad 1 \mapsto e^{i \varphi}, \quad \tau \mapsto e^{-i \varphi} .
\end{aligned}
$$

- Morphisms:

$$
\begin{aligned}
\operatorname{Mor}\left(x_{1}, x_{1}\right) & =\left\{\left(\mathrm{id},\left[\operatorname{cst}_{x_{1}}\right]\right)\right\} \cong\{1\} \\
\operatorname{Mor}\left(x_{1}, x_{-1}\right) & =\emptyset \\
\operatorname{Mor}\left(x_{1}, y_{\varphi}\right) & =\emptyset \\
\operatorname{Mor}\left(x_{-1}, x_{1}\right) & =\emptyset \\
\operatorname{Mor}\left(x_{-1}, x_{-1}\right) & =\left\{\left(\mathrm{id},\left[\operatorname{cst}_{x_{-1}}\right]\right)\right\} \cong\{1\} \\
\operatorname{Mor}\left(x_{-1}, y_{\varphi}\right) & =\emptyset \\
\operatorname{Mor}\left(y_{\varphi}, x_{1}\right) & =\left\{\left(\operatorname{pr}, s^{n} v_{\varphi}^{-1}\right) \mid n \in \mathbb{Z}\right\} \cong \mathbb{Z} \\
\operatorname{Mor}\left(y_{\varphi}, x_{-1}\right) & =\left\{\left(\operatorname{pr}, v_{x_{-1}} s^{n} v_{\varphi}^{-1}\right) \mid n \in \mathbb{Z}\right\} \cong \mathbb{Z} \\
\operatorname{Mor}\left(y_{\varphi}, y_{\varphi^{\prime}}\right) & =\left\{\left(\mathrm{id}, v_{\varphi^{\prime}} s^{n} v_{\varphi}^{-1}\right) \mid n \in \mathbb{Z}\right\} \cup\left\{\left(\tau, \tau^{*}\left(v_{\varphi^{\prime}}\right) s^{n} v_{\varphi}^{-1}\right) \mid n \in \mathbb{Z}\right\}
\end{aligned}
$$

Here $s, v_{\varphi}, v_{x_{-1}}: G /\{1\} \times I \rightarrow S^{1}$ are paths. Their value at $1 \in G$ is as follows: $s$ denotes the path starting at $1 \in S^{1}$ and going once around $S^{1}, v_{\varphi}$ denotes the path starting at $1 \in S^{1}$ and going to $e^{i \varphi}$ and $v_{x_{-1}}$ denotes the path starting at $1 \in S^{1}$ and going to $-1 \in S^{1}$, all counterclockwise. Their value at $\tau \in G$ is the reflection of these respective paths. 
On the objects of the category, $\phi:=\Pi(G, f)$ is given by

$$
\begin{aligned}
x_{1} & \mapsto x_{1} \\
x_{-1} & \mapsto \begin{cases}x_{-1} & \text { if } d \text { is odd } \\
x_{1} & \text { if } d \text { is even }\end{cases} \\
y_{\varphi} & \mapsto y_{d \cdot \varphi} .
\end{aligned}
$$

On the morphisms, it is given by the identity on the morphisms originating at $x_{1}$ and $x_{-1}$, and by multiplication with $d$ (on the path factor) on the morphisms originating at $y_{\varphi}$.

A $\mathbb{Z} / 2$-CW-structure on $S^{1}$ is given by: Two 0-cells of type $\mathbb{Z} / 2$, namely $x_{1}$ and $x_{-1}$, and one 1 -cell of type $\{1\}$, namely $a: \mathbb{Z} / 2 \times I \rightarrow S^{1},(1, t) \mapsto$ $e^{\pi i t},(\tau, t) \mapsto e^{-\pi i t}$.

An $\operatorname{Aut}\left(y_{\phi}\right)$-CW-structure on $\widetilde{S^{1}\left(y_{\phi}\right)}$ is given by: Two 0-cells of type $\mathbb{Z} / 2$, namely

$$
\begin{aligned}
\widetilde{x_{1}}: \mathbb{Z} & \rightarrow \mathbb{R}, n \mapsto n \\
\widetilde{x_{-1}}: \mathbb{Z} & \rightarrow \mathbb{R}, n \mapsto n+\frac{1}{2},
\end{aligned}
$$

and one 1-cell of type $\{1\}$, namely

$$
\begin{aligned}
\widetilde{a}: \operatorname{Aut}(y) \times I & \rightarrow \mathbb{R} \\
((1, n), t) & \mapsto n+\frac{t}{2} \\
((\tau, n), t) & \mapsto(n+1)-\frac{t}{2} .
\end{aligned}
$$

We have $\widetilde{S^{1}\left(x_{1}\right)}=\mathrm{pt}=\widetilde{S^{1}\left(x_{-1}\right)}$. What is $C^{c}\left(\widetilde{S^{1}}\right)$ ?

$$
\begin{aligned}
C_{i}^{c}\left(\widetilde{S^{1}}\right)\left(x_{1}\right) & = \begin{cases}R & \text { if } i=0 \\
0 & \text { if } i \neq 0\end{cases} \\
C_{i}^{c}\left(\widetilde{S^{1}}\right)\left(x_{-1}\right) & = \begin{cases}R & \text { if } i=0 \\
0 & \text { if } i \neq 0\end{cases} \\
C_{i}^{c}\left(\widetilde{S^{1}}\right)\left(y_{\varphi}\right) & = \begin{cases}R \mathbb{Z} \oplus R \mathbb{Z} & \text { if } i=0 \\
R \operatorname{Aut}\left(y_{\varphi}\right) & \text { if } i=1 \\
0 & \text { if } i \neq 0,1\end{cases}
\end{aligned}
$$

When looking at $C^{c}(\widetilde{f})$, we have to distinguish two cases, $d$ even and $d$ odd. Assume that $d$ is odd. Then

$$
\begin{aligned}
C^{c}(\widetilde{f})\left(x_{1}\right) & =\operatorname{id}_{R}: C^{c}\left(\widetilde{S^{1}}\right)\left(x_{1}\right) \rightarrow C^{c}\left(\widetilde{S^{1}}\right)\left(x_{1}\right) \\
C^{c}(\widetilde{f})\left(x_{-1}\right) & =\operatorname{id}_{R}: C^{c}\left(\widetilde{S^{1}}\right)\left(x_{-1}\right) \rightarrow C^{c}\left(\widetilde{S^{1}}\right)\left(x_{-1}\right) \\
C^{c}(\widetilde{f})\left(y_{\varphi}\right) & =\left(\operatorname{id}_{R} \cdot(\cdot d), \operatorname{id}_{R} \cdot\left(\cdot d+\frac{d-1}{2}\right), d^{\prime}\right): C^{c}\left(\widetilde{S^{1}}\right)\left(y_{\varphi}\right) \rightarrow C^{c}\left(\widetilde{S^{1}}\right)\left(y_{d \cdot \varphi}\right),
\end{aligned}
$$


where

$$
\begin{aligned}
d^{\prime}: R \operatorname{Aut}\left(y_{\varphi}\right) \rightarrow & R \operatorname{Aut}\left(y_{d \varphi}\right) \\
(1, m) \mapsto & (1, d m)+(1, d m+1)+\ldots+\left(1, d m+\frac{d-1}{2}\right) \\
& -\left((\tau, d m)+(\tau, d m+1)+\ldots+\left(\tau, d m+\frac{d-3}{2}\right)\right) \\
(\tau, m) \mapsto & \left(\tau, d m+\frac{d-1}{2}\right)+\ldots+(\tau, d m+d-1) \\
& -\left(\left(1, d m+\frac{d+1}{2}\right)+\ldots+(1, d m+d-1)\right)
\end{aligned}
$$

If $d$ is even, we obtain

$$
\begin{aligned}
C^{c}(\widetilde{f})\left(x_{1}\right) & =\operatorname{id}_{R}: C^{c}\left(\widetilde{S^{1}}\right)\left(x_{1}\right) \rightarrow C^{c}\left(\widetilde{S^{1}}\right)\left(x_{1}\right) \\
C^{c}(\widetilde{f})\left(x_{-1}\right) & =\operatorname{id}_{R}: C^{c}\left(\widetilde{S^{1}}\right)\left(x_{-1}\right) \rightarrow C^{c}\left(\widetilde{S^{1}}\right)\left(x_{1}\right) \\
C^{c}(\widetilde{f})\left(y_{\varphi}\right) & =\left(\left(\begin{array}{cc}
\operatorname{id}_{R} \cdot(\cdot d) & \operatorname{id}_{R} \cdot\left(\cdot d+\frac{d}{2}\right) \\
0 & 0
\end{array}\right), d^{\prime \prime}\right): C^{c}\left(\widetilde{S^{1}}\right)\left(y_{\varphi}\right) \rightarrow C^{c}\left(\widetilde{S^{1}}\right)\left(y_{d \cdot \varphi}\right),
\end{aligned}
$$

where

$$
\begin{aligned}
d^{\prime \prime}: R \operatorname{Aut}\left(y_{\varphi}\right) \rightarrow & R \operatorname{Aut}\left(y_{d \varphi}\right) \\
(1, m) \mapsto & (1, d m)+(1, d m+1)+\ldots+\left(1, d m+\frac{d-2}{2}\right) \\
& -\left((\tau, d m)+(\tau, d m+1)+\ldots+\left(\tau, d m+\frac{d-2}{2}\right)\right) \\
(\tau, m) \mapsto & \left(\tau, d m+\frac{d}{2}\right)+\ldots+(\tau, d m+d-1) \\
& -\left(\left(1, d m+\frac{d}{2}\right)+\ldots+(1, d m+d-1)\right)
\end{aligned}
$$

We have

$$
U_{\mathbb{Z} / 2}^{R}\left(S^{1}, f\right)=K_{0}\left(\phi-\operatorname{end}_{\mathrm{ff} R \Pi\left(\mathbb{Z} / 2, S^{1}\right)}\right)
$$

and $u_{G}^{R}\left(S^{1}, f\right)=\left[C^{c}(\widetilde{f})\right]$, so if $d$ is odd, then

$u_{G}^{R}\left(S^{1}, f\right)=\left[\left(\operatorname{id}_{R}\right)_{x_{1}} \oplus\left(\operatorname{id}_{R}\right)_{x_{-1}} \oplus\left(\operatorname{id}_{R} \cdot(\cdot d) \oplus \operatorname{id}_{R} \cdot\left(\cdot d+\frac{d-1}{2}\right)\right)_{y_{\varphi}}\right]-\left[\left(d^{\prime}\right)_{y_{\varphi}}\right]$,

if $d$ is even, then

$u_{G}^{R}\left(S^{1}, f\right)=\left[\left(\operatorname{id}_{R}\right)_{x_{1}} \oplus\left(\operatorname{id}_{R}\right)_{x_{-1}} \oplus\left(\begin{array}{cc}\operatorname{id}_{R} \cdot(\cdot d) & \operatorname{id}_{R} \cdot\left(\cdot d+\frac{d}{2}\right) \\ 0 & 0\end{array}\right)_{y_{\varphi}}\right]-\left[\left(d^{\prime \prime}\right)_{y_{\varphi}}\right]$.

This example is continued in Section 6.3, where the splitting results of Chapters 5 and 6 are taken into account. 


\subsection{Functoriality}

Let $(X, f)$ and $(Y, g)$ be finite proper $G$-CW-complexes with $G$-equivariant cellular endomorphisms $f$ and $g$. Let $h: X \rightarrow Y$ be a $G$-equivariant cellular map such that $g h=h f$. Then composition with $h$ induces a functor $H:=$ $\Pi(G, h): \Pi(G, X) \rightarrow \Pi(G, Y)$ between the fundamental categories. Setting $\phi:=\Pi(G, f)$ and $\psi:=\Pi(G, g)$, the diagram

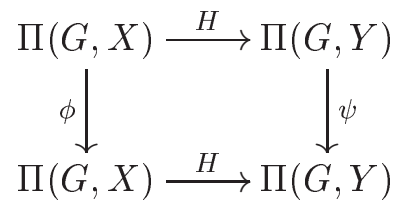

commutes. We are in the algebraic situation of Section 2.2 and obtain a group homomorphism

$$
U_{G}^{R}(h):=K_{0}\left(H_{*}\right): U_{G}^{R}(X, f) \rightarrow U_{G}^{R}(Y, g) .
$$

Thus $U_{G}^{R}$ is a functor from $\operatorname{End}\left(G-\mathcal{C} W_{\mathrm{fp}}\right)$ to $\mathcal{A} b$. But we know even more:

Lemma 3.3.1. If $h:(X, f) \rightarrow(Y, g)$ is a $G$-equivariant cellular map between finite proper $G$-CW-complexes with endomorphisms, i.e., such that $g h=h f$, then we have a commutative diagram

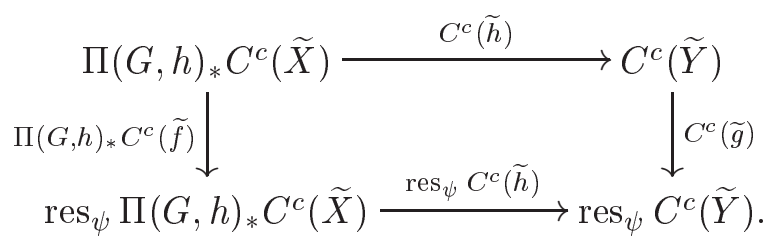

Proof. The map $h: X \rightarrow Y$ yields a natural transformation $\widetilde{h}: \widetilde{X} \rightarrow \widetilde{Y}$ 。 $\Pi(G, h)$, thus also [Lüc89, 13.4]

$$
C^{c}(\widetilde{h}): C^{c}(\widetilde{X}) \rightarrow C^{c}(\widetilde{Y} \circ \Pi(G, h))=\operatorname{res}_{\Pi(G, h)} C^{c}(\widetilde{Y}) .
$$

In accordance with our usual conventions, we set $H:=\Pi(G, h), \phi:=\Pi(G, f)$ and $\psi:=\Pi(G, g)$. If $h$ is a morphism such that $g h=h f$, we obtain a commutative diagram of $R \Pi(G, X)$-chain complexes

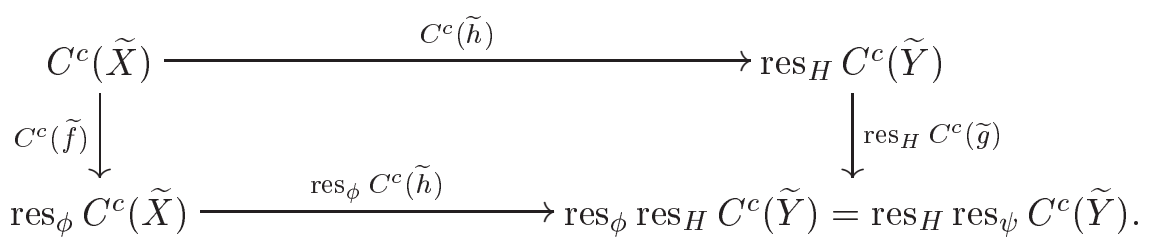


By adjunction, the above yields a commutative diagram

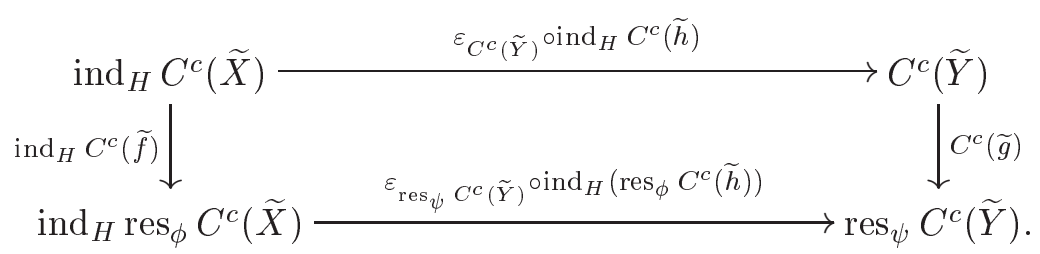

We remember that $H_{*}\left(C^{c}(\widetilde{f})\right)$ was not exactly $\operatorname{ind}_{H} C^{c}(\widetilde{f})$. Abbreviating res by $r$ and ind by $i$ for better readability, we use the commutative diagram

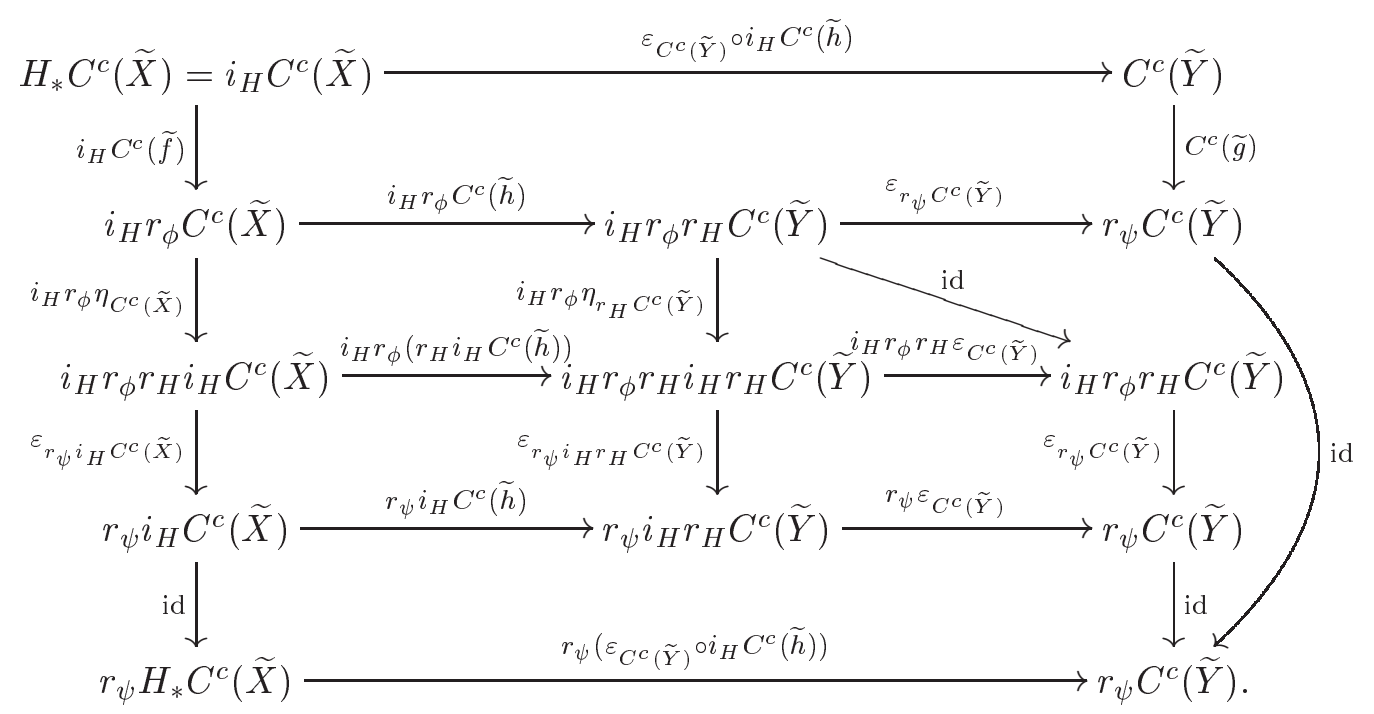

to establish

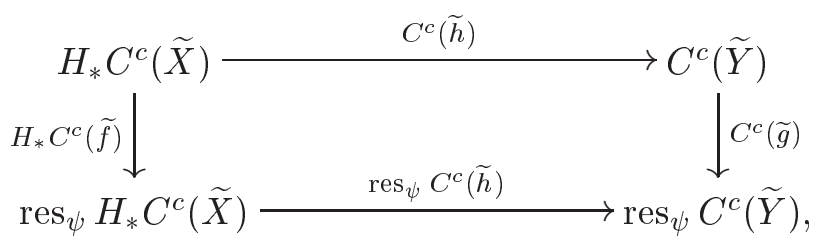

where we defined $C^{c}(\widetilde{h}):=\varepsilon_{C^{c}(\widetilde{Y})} \circ \operatorname{ind}_{H} C^{c}(\widetilde{h})$. We denote both the map $H_{*} C^{c}(\widetilde{X}) \rightarrow C^{c}(\widetilde{Y})$ and the map $C^{c}(\widetilde{X}) \rightarrow \operatorname{res}_{H} C^{c}(\tilde{Y})$ by $C^{c}(\widetilde{h})$ since they determine each other and no confusion can occur.

Lemma 3.3.1 implies that $C^{c}(\widetilde{h})$ is a map from $\Pi(G, h)_{*} C^{c}(\widetilde{f})$ to $C^{c}(\widetilde{g})$.

\subsection{The Induction Structure}

Let $X$ be an $H$-CW-complex with $H$-equivariant endomorphism $f: X \rightarrow X$ and let $\alpha: H \rightarrow G$ be a group homomorphism. Then $\operatorname{ind}_{\alpha} X:=G \times_{H} X$ 
is a $G$-CW-complex. It is proper if $X$ is proper. (If $K \leq H$ is finite, then for any group homomorphism $\alpha: H \rightarrow G$ also $\alpha(K)$ is finite.) The map $\operatorname{ind}_{\alpha}: X=H \times_{H} X \stackrel{\alpha \times \text { id }}{\longrightarrow} G \times_{H} X=\operatorname{ind}_{\alpha} X$ (which can also be seen as $\left.X \rightarrow 1 \times_{\operatorname{ker}(\alpha)} X \subseteq G \times_{H} X\right)$ induces a map $\Pi\left(\operatorname{ind}_{\alpha}\right)$ of the fundamental categories:

$$
\begin{aligned}
\Pi\left(\operatorname{ind}_{\alpha}\right): & \\
\Pi(H, X) & \rightarrow \Pi\left(G, \operatorname{ind}_{\alpha} X\right) \\
(x: H / K \rightarrow X) & \mapsto\left(\operatorname{ind}_{\alpha} x: G \times_{H}(H / K)=G / \alpha(K) \stackrel{\operatorname{id}_{G} \times_{H} x}{\longrightarrow} G \times_{H} X\right) \\
(\sigma, w) & \mapsto\left(\operatorname{ind}_{\alpha} \sigma, \operatorname{ind}_{\alpha} w\right),
\end{aligned}
$$

where for $(\sigma, w) \in \operatorname{Mor}_{\Pi(H, X)}(x(K), y(L))$ we set

$$
\begin{aligned}
\operatorname{ind}_{\alpha} \sigma & =\operatorname{id}_{G} \times_{H} \sigma: G \times_{H}(H / K)=G / \alpha(K) \rightarrow G \times_{H}(H / L)=G / \alpha(L), \\
\operatorname{ind}_{\alpha} w & =\operatorname{id}_{G} \times_{H} w: G / \alpha(K) \times I=G \times_{H}(G / K \times I) \rightarrow G \times_{H} X .
\end{aligned}
$$

We set $\operatorname{ind}_{\alpha} f=\operatorname{id}_{G} \times_{H} f: G \times_{H} X \rightarrow G \times_{H} X$. The diagram

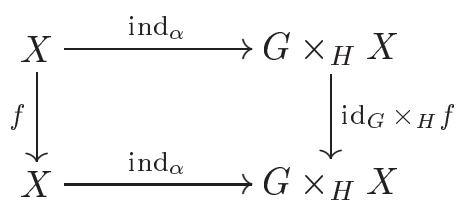

commutes since ind $\operatorname{in}_{\alpha}$ is a functor and $f$ is $H$-equivariant. Setting $A:=$ $\Pi\left(\operatorname{ind}_{\alpha}\right), \phi:=\Pi(H, f)$ and $\psi:=\Pi\left(G, \operatorname{ind}_{\alpha} f\right)$, the diagram

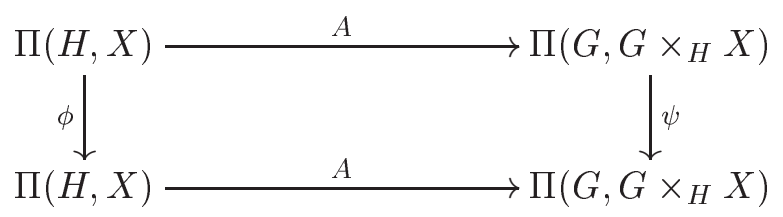

also commutes, and we obtain a group homomorphism

$$
\alpha_{*}:=U^{R}(\alpha):=K_{0}\left(A_{*}\right): U_{H}^{R}(X, f) \rightarrow U_{G}^{R}\left(\operatorname{ind}_{\alpha} X, \operatorname{ind}_{\alpha} f\right) .
$$

\subsection{Properties of the Geometric Invariant}

We defined the invariant $\left(U_{G}^{R}(X, f), u_{G}^{R}(X, f)\right)$ because it has certain nice properties. Namely, it is a functorial equivariant Lefschetz invariant, which means that it satisfies additivity, $G$-homotopy invariance, invariance under $G$-homotopy equivalence and is normalized. We define a functorial equivariant Lefschetz invariant in Definition 3.5.1, in analogy to [Lüc99, 2.3], where the non-equivariant case is treated, and to [LR03, Lemma 3.7], where a coarser equivariant invariant is studied. 
Definition 3.5.1. A functorial equivariant Lefschetz invariant on the family of categories $G-\mathcal{C} W_{\mathrm{fp}}$ of finite proper $G$-CW-complexes for discrete groups $G$ is a pair $(\Theta, \theta)$ that consists of

- A family $\Theta$ of functors

$$
\Theta_{G}: \operatorname{End}\left(G-\mathcal{C} W_{\mathrm{fp}}\right) \rightarrow \mathcal{A} b
$$

which is compatible with the induction structure, i.e., for an inclusion $\alpha: G \rightarrow K$ there is a group homomorphism $\Theta(\alpha): \Theta_{G}(X, f) \rightarrow$ $\Theta_{K}\left(\operatorname{ind}_{\alpha} X, \operatorname{ind}_{\alpha} f\right)$ for every $(X, f) \in \operatorname{End}\left(G-\mathcal{C} W_{\text {fp }}\right)$. We want the equation $\Theta(\alpha) \Theta_{G}(h)=\Theta_{K}\left(\operatorname{ind}_{\alpha} h\right) \Theta(\alpha)$ to hold for any morphism $h:(X, f) \rightarrow(Y, g)$.

- A family $\theta$ of functions $\theta_{G}:(X, f) \mapsto \theta_{G}(X, f) \in \Theta_{G}(X, f)$.

such that the following holds:

1. Additivity

For a $G$-pushout with $i_{2}$ a $G$-cofibration

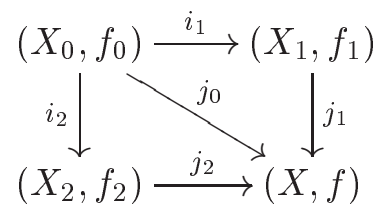

we obtain in $\Theta_{G}(X, f)$ that

$\theta_{G}(X, f)=\Theta_{G}\left(j_{1}\right) \theta_{G}\left(X_{1}, f_{1}\right)+\Theta_{G}\left(j_{2}\right) \theta_{G}\left(X_{2}, f_{2}\right)-\Theta_{G}\left(j_{0}\right) \theta_{G}\left(X_{0}, f_{0}\right)$.

2. $G$-Homotopy invariance

If $h_{0}, h_{1}:(X, f) \rightarrow(Y, g)$ are two $G$-maps that are $G$-homotopic in $\operatorname{End}\left(G-\mathcal{C} W_{\mathrm{fp}}\right)$, then

$$
\Theta_{G}\left(h_{0}\right)=\Theta_{G}\left(h_{1}\right): \Theta_{G}(X, f) \rightarrow \Theta_{G}(Y, g) .
$$

3. Invariance under $G$-homotopy equivalence

If $h:(X, f) \rightarrow(Y, g)$ is a morphism in $\operatorname{End}\left(G-\mathcal{C} W_{\mathrm{fp}}\right)$ such that $h: X \rightarrow$ $Y$ is a $G$-homotopy equivalence, then

$$
\begin{aligned}
\Theta_{G}(h): \Theta_{G}(X, f) & \cong \Theta_{G}(Y, g) \\
\theta_{G}(X, f) & \mapsto \theta_{G}(Y, g) .
\end{aligned}
$$

4. Normalization: We have $\theta_{G}\left(\emptyset, \mathrm{id}_{\emptyset}\right)=0 \in \Theta_{G}\left(\emptyset, \operatorname{id}_{\emptyset}\right)$.

5. If $\alpha: G \rightarrow K$ is an inclusion of groups, then

$$
\alpha_{*} \theta_{G}(X, f)=\theta_{K}\left(\operatorname{ind}_{\alpha} X, \operatorname{ind}_{\alpha} f\right) \in \Theta_{K}\left(\operatorname{ind}_{\alpha} X, \operatorname{ind}_{\alpha} f\right) .
$$


A natural transformation $\tau:(\Theta, \theta) \rightarrow(\Xi, \xi)$ of functorial equivariant Lefschetz invariants is a family of natural transformations $\tau_{G}: \Theta_{G} \rightarrow \Xi_{G}$ of functors from $\operatorname{End}\left(G-\mathcal{C} W_{\mathrm{fp}}\right)$ to $\mathcal{A} b$ for discrete groups $G$ that preserves all structure.

In the non-equivariant case, a functorial Lefschetz invariant has to satisfy additivity, homotopy invariance, invariance under homotopy equivalence and normalization [Lüc99, Chapter 2, Definition 2.3]. This is the natural equivariant generalization. We write either $(\Theta, \theta),\left(\Theta_{G}, \theta_{G}\right)$ or $\left(\Theta_{G}(X, f), \theta_{G}(X, f)\right)$ for a functorial equivariant Lefschetz invariant.

We show that the invariant $\left(U_{G}^{R}(X, f), u_{G}^{R}(X, f)\right)$ that was defined in Definition 3.1.1 has these properties:

Proposition 3.5.2. The invariant $\left(U_{G}^{R}(X, f), u_{G}^{R}(X, f)\right)$ is a functorial equivariant Lefschetz invariant on the family of categories $G-\mathcal{C} W_{\mathrm{fp}}$ of finite proper $G$-CW-complexes for discrete groups $G$.

Proof. We have an abelian group $U_{G}^{R}(X, f)$ for all $(X, f)$ and for all discrete groups $G$. In Section 3.3 we defined $U_{G}^{R}(h): U^{R}(X, f) \rightarrow U_{G}^{R}(Y, g)$ for a map $h:(X, f) \rightarrow(Y, g)$. So $U_{G}^{R}$ is a functor on $\operatorname{End}\left(G-\mathcal{C} W_{\mathrm{fp}}\right)$. In Section 3.4 we defined $U_{G}^{R}(\alpha)$ for a group homomorphism $\alpha: G \rightarrow K$. We know that the diagram

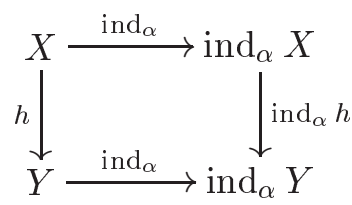

commutes, so

$$
\begin{aligned}
U(\alpha) U_{G}(h) & =\Pi\left(\operatorname{ind}_{\alpha}\right)_{*} \Pi(G, h)_{*} \\
& =\Pi\left(K, \operatorname{ind}_{\alpha} h\right)_{*} \Pi\left(\operatorname{ind}_{\alpha}\right)_{*} \\
& =U_{K}\left(\operatorname{ind}_{\alpha} h\right) U(\alpha) .
\end{aligned}
$$

The function $u_{G}^{R}$ is defined as $u_{G}^{R}:(X, f) \mapsto u_{G}^{R}(X, f):=u\left(C^{c}(\widetilde{f})\right)$. We need to show that $\left(U_{G}^{R}, u_{G}^{R}\right)$ fulfills properties $1-5$. We leave out the index $R$ in the sequel if no confusion can occur.

1. Additivity:

From [Lüc89, Lemma 13.7] one knows that for a $G$-pushout

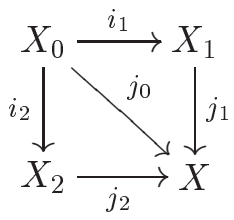


with $i_{2}$ a $G$-cofibration we obtain a based exact sequence of $R \Pi(G, X)$ chain complexes

$$
\begin{gathered}
0 \rightarrow j_{0 *} C^{c}\left(\widetilde{X_{0}}\right) \stackrel{j_{1 *} C^{c}\left(\widetilde{i_{1}}\right) \oplus j_{2 *} C^{c}\left(\widetilde{i_{2}}\right)}{\longrightarrow} j_{1 *} C^{c}\left(\widetilde{X_{1}}\right) \oplus j_{2 *} C^{c}\left(\widetilde{X_{2}}\right) \\
\stackrel{C^{c}\left(\widetilde{j_{1}}\right)-C^{c}\left(\widetilde{j_{2}}\right)}{\longrightarrow} C^{c}(\widetilde{X}) \rightarrow 0,
\end{gathered}
$$

where $j_{0 *}, j_{1 *}$ and $j_{2 *}$ denote induction with $j_{0}, j_{1}$ and $j_{2}$ respectively. Since by Lemma 3.3.1 every map $h:(X, f) \rightarrow(Y, g)$ between finite proper $G$-CW-complexes with endomorphisms induces a commutative diagram

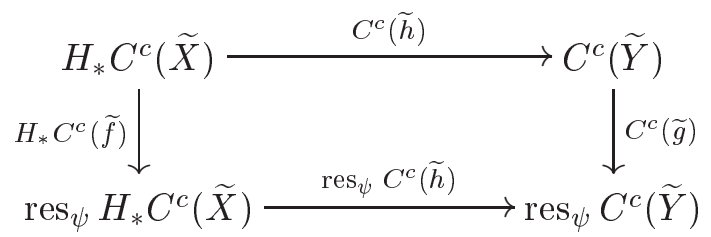

and $i_{1}, i_{2}, j_{1}$ and $j_{2}$ are such maps, we obtain a commutative diagram

$$
\begin{aligned}
& 0 \longrightarrow j_{0 *} C^{c}\left(\widetilde{X_{0}}\right) \longrightarrow j_{1 *} C^{c}\left(\widetilde{X_{1}}\right) \oplus j_{2 *} C^{c}\left(\widetilde{X_{2}}\right) \longrightarrow C^{c}(\widetilde{X}) \longrightarrow 0 \\
& \quad j_{0 *} C^{c}\left(\widetilde{f_{0}}\right) \downarrow \\
& 0 \rightarrow \operatorname{res}_{\phi} j_{0 *} C^{c}\left(\widetilde{X_{0}}\right) \rightarrow \operatorname{res}_{\phi} j_{1 *} C^{c}\left(\widetilde{X_{1}}\right) \downarrow
\end{aligned}
$$

thus a short exact sequence of $\phi$-endomorphisms of $R \Pi(G, X)$-chain complexes. From the additive properties of the algebraic invariant $(U, u)$ defined in Section 2.1 we conclude

$$
\begin{aligned}
u\left(C^{c}(\widetilde{f})\right) & =u\left(j_{1 *} C^{c}\left(\widetilde{f}_{1}\right)\right)+u\left(j_{2 *} C^{c}\left(\widetilde{f}_{2}\right)\right)-u\left(j_{0 *} C^{c}\left(\widetilde{f}_{0}\right)\right) \\
& =U\left(j_{1}\right) u\left(C^{c}\left(\widetilde{f}_{1}\right)\right)+U\left(j_{2}\right) u\left(C^{c}\left(\widetilde{f}_{2}\right)\right)-U\left(j_{0}\right) u\left(C^{c}\left(\widetilde{f}_{0}\right)\right),
\end{aligned}
$$

SO

$u_{G}(X, f)=U_{G}\left(j_{1}\right) u_{G}\left(X_{1}, f_{1}\right)+U_{G}\left(j_{2}\right) u_{G}\left(X_{2}, f_{2}\right)-U_{G}\left(j_{0}\right) u_{G}\left(X_{0}, f_{0}\right)$.

2. G-Homotopy invariance:

Let $h_{0} \simeq_{G} h_{1}:(X, f) \rightarrow(Y, g)$, where the $G$-equivariant homotopy between $h_{0}$ and $h_{1}$ is given by $H: X \times I \rightarrow Y$ satisfying the equation $g H=H\left(f \times \mathrm{id}_{I}\right)$ on $X \times I$, where $I=[0,1]$ denotes the unit interval endowed with the trivial action of $G$ and where $X \times I$ is endowed with the $G$-equivariant endomorphism $f \times \mathrm{id}_{I}$. Then we have a commutative diagram of natural transformations between categories with 
endofunctors

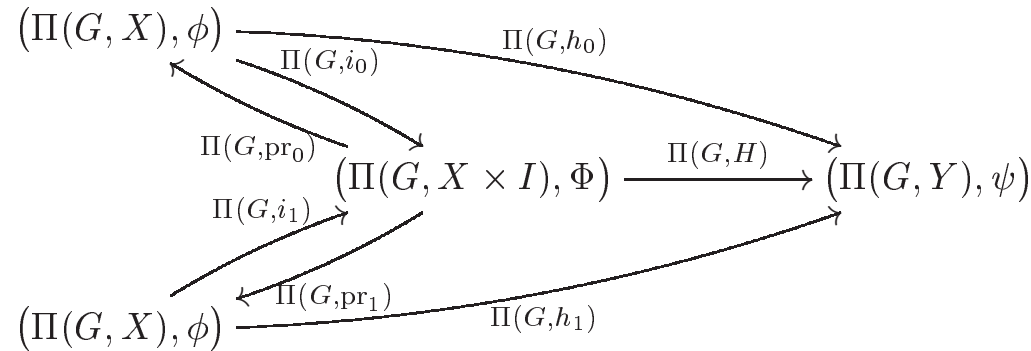

where $\phi:=\Pi(G, f), \Phi:=\Pi\left(G, f \times \operatorname{id}_{I}\right)$ and $\psi:=\Pi(G, g)$. The maps $i_{0}$ and $i_{1}$ induce equivalences of categories $\Pi\left(G, i_{0}\right)$ and $\Pi\left(G, i_{1}\right)$ from $\Pi(G, X)$ to $\Pi(G, X \times I)$. Furthermore we have $\mathrm{pr}_{0} \circ i_{0}=\mathrm{id}_{X}$, so $\Pi\left(G, \operatorname{pr}_{0}\right) \circ \Pi\left(G, i_{0}\right)=\Pi\left(G, \operatorname{id}_{X}\right)$. We know that $\Pi\left(G, \operatorname{id}_{X}\right)=$ $\operatorname{id}_{\Pi(G, X)}$ since $\Pi(G,-)$ is a functor. Analogously we obtain $\Pi\left(G, \operatorname{pr}_{1}\right) \circ$ $\Pi\left(G, i_{1}\right)=\Pi\left(G, \operatorname{id}_{X}\right)=\operatorname{id}_{\Pi(G, X)}$.

We want to show that $U_{G}\left(h_{0}\right)=U_{G}\left(h_{1}\right)$, i.e., that we have the equation $K_{0}\left(\Pi\left(G, h_{0}\right)_{*}\right)([g])=K_{0}\left(\Pi\left(G, h_{1}\right)_{*}\right)([g])$ for all $g \in \phi-\operatorname{end}_{\mathrm{ff} R \Pi(G, X)}$. We know that $\Pi\left(G, h_{0}\right)=\Pi(G, H) \circ \Pi\left(G, i_{0}\right)$ and that $\Pi\left(G, h_{1}\right)=$ $\Pi(G, H) \circ \Pi\left(G, i_{1}\right)$, so it suffices to prove that $K_{0}\left(\Pi\left(G, i_{0}\right)_{*}(g)\right)=$ $K_{0}\left(\Pi\left(G, i_{1}\right)_{*}(g)\right)$ for all $g \in \phi-\operatorname{end}_{\mathrm{ff} R \Pi(G, X)}$.

This is shown if for $(g: M \rightarrow M \circ \phi) \in \phi-\operatorname{end}_{\mathrm{ff} R \Pi(G, X)}$ we find a natural equivalence

$$
t: \operatorname{ind}_{\Pi\left(G, i_{0}\right)} \stackrel{\sim}{\rightarrow} \operatorname{ind}_{\Pi\left(G, i_{1}\right)}
$$

such that

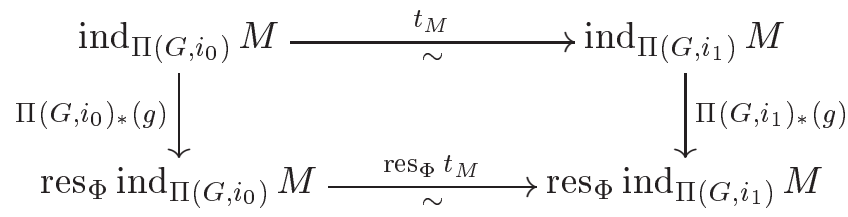

commutes. We define the map $t$ by

$$
\begin{aligned}
t_{M}: & \operatorname{ind}_{\Pi\left(G, i_{0}\right)} M=M(?) \otimes_{R \Pi(G, X)} R \Pi(G, X \times I)\left(? ?, \Pi\left(G, i_{0}\right)(?)\right) \\
& \stackrel{\operatorname{id}_{M} \otimes t^{\prime}}{\longrightarrow} M \otimes_{R \Pi(G, X)} R \Pi(G, X \times I)\left(? ?, \Pi\left(G, i_{1}\right)(?)\right)=\operatorname{ind}_{\Pi\left(G, i_{1}\right)} M,
\end{aligned}
$$

where for $?=(x: G / H \rightarrow X) \in \Pi(G, X)$ we have

$$
\begin{aligned}
& \Pi\left(G, i_{0}\right)(x)=i_{0} \circ x: G / H \stackrel{x}{\rightarrow} X \stackrel{i_{0}}{\rightarrow} X \times I \\
& \text { and } \quad \Pi\left(G, i_{1}\right)(x)=i_{1} \circ x: G / H \stackrel{x}{\rightarrow} X \stackrel{i_{1}}{\rightarrow} X \times I,
\end{aligned}
$$

and where $t_{x}^{\prime}: R \Pi(G, X \times I)\left(? ?, i_{0} \circ x\right) \rightarrow R \Pi(G, X \times I)\left(? ?, i_{1} \circ x\right)$ is given by composition with the morphism $\left(\operatorname{id}_{G / H}, v_{x}\right) \in R \Pi(G, X \times$ 
$I)\left(i_{0} \circ x, i_{1} \circ x\right)$ where $v_{x}$ is the path from $i_{1} \circ x$ to $i_{0} \circ x$ given by the map $x \times\left(1-\operatorname{id}_{I}\right): G / H \times I \rightarrow X \times I$. By definition $t$ is a natural transformation. An inverse to $t_{x}^{\prime}$ can be given by composition with $\left(\mathrm{id}_{G / H}, v_{x}^{-1}\right)$. So $t=\mathrm{id}_{M} \otimes t^{\prime}$ has the inverse $\mathrm{id}_{M} \otimes t^{\prime-1}$ and is a natural equivalence. The following diagram commutes by naturality:

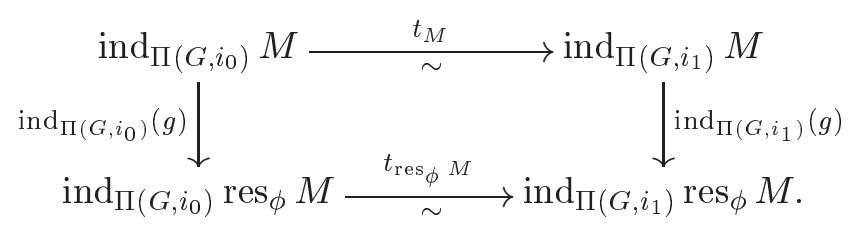

The precomposition with the morphism $\left(\operatorname{id}_{G / H}, v_{x}\right)$ gives us a map $s_{x}^{\prime}: R \Pi(G, X \times I)\left(\Pi\left(G, i_{1}\right)(x), ? ?\right) \rightarrow R \Pi(G, X \times I)\left(\Pi\left(G, i_{0}\right)(x), ? ?\right)$, so we have a natural transformation of functors $\operatorname{id}_{M} \otimes s^{\prime}: \operatorname{res}_{\Pi\left(G, i_{1}\right)} M \rightarrow$ $\operatorname{res}_{\Pi\left(G, i_{0}\right)} M$. The inverse of $s_{x}^{\prime}$ is precomposition with $\left(\mathrm{id}_{G / H}, v_{x}^{-1}\right)$, so $s$ is also a natural equivalence.

One checks commutativity of the following diagram, where we call $\Pi\left(G, i_{0}\right)=I_{0}$ and $\Pi\left(G, i_{1}\right)=I_{1}$ for simplicity of notation, and where we abbreviate res by $r$ and ind by $i$.

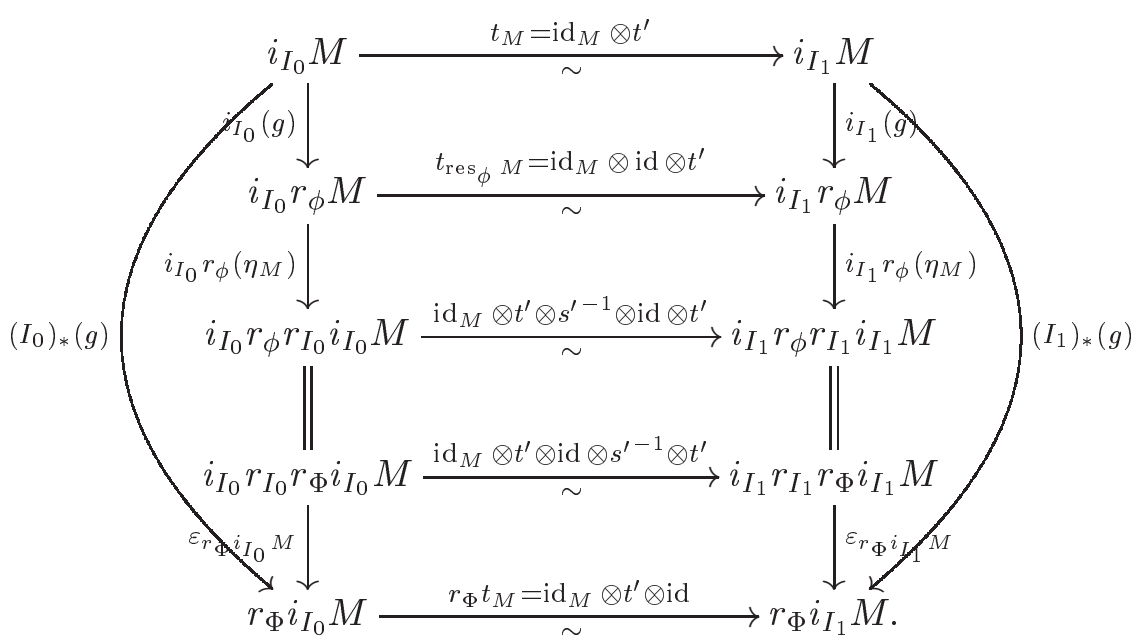

So $t$ is a natural equivalence, $t_{M}:\left(I_{0}\right)_{*}(g) \stackrel{\sim}{\rightarrow}\left(I_{1}\right)_{*}(g)$, which implies $\left[\left(I_{0}\right)_{*}(g)\right]=\left[\left(I_{1}\right)_{*}(g)\right]$. For all $[g] \in U_{G}(X, f)$ we have

$$
\begin{aligned}
U_{G}\left(h_{0}\right)([g]) & =K_{0}\left(\Pi\left(G, h_{0}\right)_{*}\right)([g])=K_{0}\left(\Pi\left(G, h_{0}\right)_{*}(g)\right) \\
& =K_{0}\left(\Pi\left(G, h_{1}\right)_{*}(g)\right)=K_{0}\left(\Pi\left(G, h_{1}\right)_{*}\right)([g]) \\
& =U_{G}\left(h_{1}\right)([g]),
\end{aligned}
$$

thus $U_{G}\left(h_{0}\right)=U_{G}\left(h_{1}\right)$ as was to be shown. 
3. Invariance under $G$-homotopy equivalence:

If $h:(X, f) \rightarrow(Y, g) \in \operatorname{End}\left(G-\mathcal{C} W_{\mathrm{fp}}\right)$ such that $h: X \rightarrow Y$ is a $G$ homotopy equivalence, then $\Pi(G, h)$ is an equivalence of categories, which implies that $\Pi(G, h)_{*}: \phi-\operatorname{end}_{\mathrm{ff} R \Pi(G, X)} \rightarrow \psi$-end $\operatorname{eff} R \Pi(G, Y)_{\text {is an }}$ equivalence of categories by Proposition 2.2.4. Thus we know that $U_{G}(h):=K_{0}\left(\Pi(G, h)_{*}\right)$ is a bijection. We need to show that $u_{G}(X, f)$ maps to $u_{G}(Y, g)$ under this map.

By Lemma 3.3.1, we know that we have a commutative diagram of finite free $R \Pi(G, X)$-chain complexes

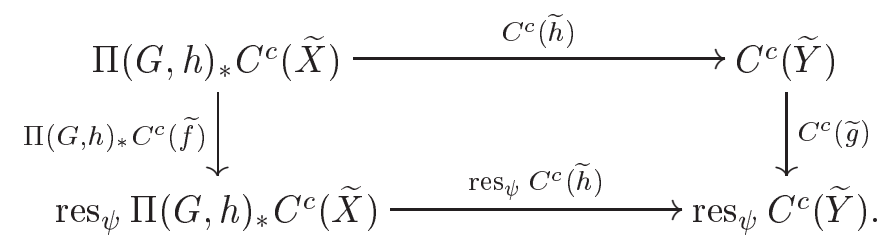

We know that the map $h: X \rightarrow Y$ is a $G$-homotopy equivalence, so there exists a $G$-homotopy inverse $l: Y \rightarrow X$, i.e., a $G$-equivariant map $l: Y \rightarrow X$ such that $l \circ h \simeq_{G} \operatorname{id}_{X}$ and $h \circ l \simeq_{G} \operatorname{id}_{Y}$. This implies that for all $K \leq G$ the restrictions to the fixed point sets $h^{K}: X^{K} \rightarrow Y^{K}$ are homotopy equivalences with homotopy inverses $l^{K}$, and thus that for all $K \leq G$ and $x: G / K \rightarrow X \in \mathrm{Ob} \Pi(G, X)$ the maps $\widehat{h^{K}(x)}: \widehat{X^{K}(x)} \rightarrow Y^{K(h(x))}$ are homotopy equivalences with homotopy inverses $\widehat{l^{K}(h(x))}$. It follows that $C^{c}\left(\widehat{h^{K}(x)}\right): C^{c}\left(\widehat{X^{K}(x)}\right) \rightarrow$ $\left.C^{c}\left(Y^{K(h(x)}\right)\right)$ is a chain homotopy equivalence for all $K \leq G$ and all $x: G / K \rightarrow X$, with homotopy inverse $\left.C^{c} \widetilde{\left(l^{K}(x)\right.}\right)$. So $C^{c}(\widetilde{h})$ is an $R \Pi(G, X)$-chain homotopy equivalence. We use Lemma 2.3.4 to conclude that $u\left(\Pi(G, h)_{*} C^{c}(\widetilde{f})\right)=u\left(C^{c}(\widetilde{g})\right)$. We obtain

$$
\begin{aligned}
U_{G}(h)\left(u_{G}(X, f)\right) & =K_{0}\left(\Pi(G, h)_{*}\right) K_{0}\left(C^{c}(\widetilde{f})\right)=K_{0}\left(\Pi(G, h)_{*} C^{c}(\widetilde{f})\right) \\
& =u\left(\Pi(G, h)_{*} C^{c}(\widetilde{f})\right)=u\left(C^{c}(\widetilde{g})\right)=u_{G}(X, g)
\end{aligned}
$$

as was to be shown.

4. Normalization: $u_{G}\left(\emptyset, \mathrm{id}_{\emptyset}\right)=0 \in U_{G}\left(\emptyset, \mathrm{id}_{\emptyset}\right)$.

We will show that $U_{G}\left(\emptyset, \operatorname{id}_{\emptyset}\right)=\{0\}$. We defined the group $U_{G}\left(\emptyset, \operatorname{id}_{\emptyset}\right)$ to be $U_{G}\left(\emptyset, \operatorname{id}_{\emptyset}\right):=U\left(R, \Pi(G, \emptyset), \operatorname{id}_{\emptyset}\right)$. We know that $\Pi(G, \emptyset)=\emptyset$, the empty category, since there exist no maps to $\emptyset$. There exists exactly one map $\emptyset \rightarrow R$-Mod, and $\operatorname{id}_{\emptyset}$-morphisms of this functor are only the identity. (Any possible morphism is an $\operatorname{id}_{\emptyset}$-morphism since any morphism commutes with the identity $\operatorname{id}_{\emptyset}$, and here only the trivial morphism is possible.) So $\operatorname{id}_{\emptyset}-e d_{f f} \not \emptyset=\{\mathrm{pt}\}$, the one-point category with only id $\mathrm{pt}_{\mathrm{pt}}$ as morphism. This implies that $K_{0}\left(\mathrm{id}_{\emptyset}\right.$-end $\left.\mathrm{ff}_{\mathrm{f} \emptyset}\right)=$ $K_{0}(\{\mathrm{pt}\})=\{0\}$. 
5. Let $\alpha: G \rightarrow K$ be an inclusion of groups. We want to show that $\alpha_{*} u_{G}(X, f)=u_{K}\left(\operatorname{ind}_{\alpha} X, \operatorname{ind}_{\alpha} f\right)$.

We know that $\alpha_{*} u_{G}(X, f)=u\left(A_{*} C^{c}(\widetilde{f})\right)$, where $A:=\Pi\left(\operatorname{ind}_{\alpha}\right)$ : $\Pi(G, X) \rightarrow \Pi\left(K, \operatorname{ind}_{\alpha} X\right)$ was defined in Section 3.4, and we also know that $u_{K}\left(\operatorname{ind}_{\alpha} X, \operatorname{ind}_{\alpha} f\right)=u\left(C^{c}\left(\widetilde{\operatorname{ind}_{\alpha} f}\right)\right)$. So we need to show that $u\left(A_{*} C^{c}(\widetilde{f})\right)=u\left(C^{c}\left(\widetilde{\operatorname{ind}_{\alpha} f}\right)\right)$. We are finished if the diagram

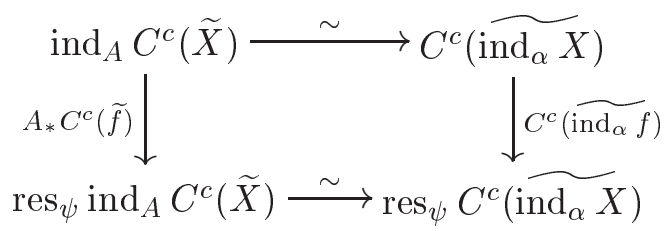

commutes. We start by showing that there is a natural equivalence

$$
T: \operatorname{ind}_{A} \tilde{X} \rightarrow \widetilde{\operatorname{ind}_{\alpha} X}
$$

of $\Pi\left(K, \operatorname{ind}_{\alpha} X\right)$-spaces.

We remember that $\widetilde{X}(x)=\widetilde{X^{H}(x)}$ is an Aut $(x)$-module. When we identify $\widetilde{X}(x)=\left\{(\mathrm{id}, v) \mid v_{1}=x\right\}$, then we can describe the action by $\widetilde{X}(x) \otimes \operatorname{Aut}(x) \rightarrow \widetilde{X}(x),(\mathrm{id}, v) \otimes(\sigma, t) \mapsto\left(\mathrm{id}, \sigma^{*} v t\right)$. This is not quite composition of paths since the composition would be $\left(\sigma, \sigma^{*} v t\right)$. This is not an element in $\widetilde{X}(x)$, we have to identify it with (id, $\sigma^{*} v t$ ). Geometrically, what we think of as a point in $\widehat{X^{H}(x)}$ is the endpoint of the path, and that stays the same. Analogously, $\widetilde{X}$ is a $\Pi(G, X)$ module by composing paths and identifying the group elements with id afterwards.

Let $\left(y: K / L \rightarrow \operatorname{ind}_{\alpha} X\right) \in \Pi\left(K, \operatorname{ind}_{\alpha} X\right)$. We define $T_{y}$ by

$$
\begin{aligned}
T_{y}: \tilde{X}(?) \otimes_{R \Pi(G, X)} R \Pi\left(K, \operatorname{ind}_{\alpha} X\right)(y, A(?)) & \rightarrow\left(\widetilde{\operatorname{ind}_{\alpha} X}\right)(y) \\
(\mathrm{id}, v) \otimes(\sigma, w) & \mapsto\left(\operatorname{id}^{*}\left(\operatorname{ind}_{\alpha} v\right) w\right) .
\end{aligned}
$$

We define an inverse $W_{y}$ of $T_{y}$ by

$$
\begin{aligned}
W_{y}:\left(\widetilde{\operatorname{ind}_{\alpha} X}\right)(y) & \rightarrow \widetilde{X}(?) \otimes_{R \Pi(G, X)} R \Pi\left(K, \operatorname{ind}_{\alpha} X\right)(y, A(?)) \\
(\mathrm{id}, s) & \mapsto\left(\mathrm{id}, \alpha^{*}\left(\sigma_{k}^{-1}\right)^{*} s\right) \otimes\left(\sigma_{k}, \operatorname{cst}_{y}\right),
\end{aligned}
$$

where $\operatorname{cst}_{y}$ denotes the constant path at $y$ and we need to explain $\sigma_{k}$. We choose $k$ such that $k . y \in G \times_{G} X \subseteq K \times_{G} X$. This is only well defined up to an element in $G$, we could have chosen any $\alpha(b) k$ for $b \in G$. But the map $W_{y}$ will turn out to be independent of this choice. We set

$$
\sigma_{k}:=\left(R_{k^{-1}}: K / L \rightarrow K / k L k^{-1}, \widetilde{k} L \mapsto \widetilde{k} k^{-1}\left(k L k^{-1}\right)\right) .
$$


We have $y \circ \sigma_{k}^{-1}=k . y \in G \times_{G} X \subseteq K \times_{G} X$. So $k . y=\operatorname{ind}_{\alpha} x$ for a unique $x \in X$. If $y \in\left(\operatorname{ind}_{\alpha} X\right)^{L}$, then $k . y \in\left(\operatorname{ind}_{\alpha} X\right)^{k L k^{-1}}$ and $k L k^{-1} \leq \alpha(G)$. Setting $H:=\alpha^{-1}\left(k L k^{-1}\right)$ we have $x \in X^{H}$. The path $\left(\sigma_{k}^{-1}\right)^{*} s=k . s$ lies in $G \times{ }_{G} X \cong X$, so its restriction $\alpha^{*}\left(\sigma_{k}^{-1}\right)^{*} s=k . s$ lies in $X$.

The definition of the map $W_{y}$ does not depend on the choice of $k$ because of the tensor product relation. For an element $b \in G$, we have in $\widetilde{X}(?) \otimes_{R \Pi(G, X)} R \Pi\left(K, \operatorname{ind}_{\alpha} X\right)(y, A(?))$ the equation

$$
\begin{aligned}
\left(\mathrm{id}, \alpha^{*}\left(\sigma_{\alpha(b) k}^{-1}\right)^{*} s\right) \otimes\left(\sigma_{\alpha(b) k}, \operatorname{cst}_{y}\right) \\
=\left(\mathrm{id}, \alpha^{*}\left(\sigma_{\alpha(b)}^{-1}\right)^{*}\left(\sigma_{k}^{-1}\right)^{*} s\right) \otimes\left(\left(\sigma_{\alpha(b)}, \operatorname{cst}_{y}\right)\left(\sigma_{k}, \operatorname{cst}_{y}\right)\right) \\
=\left(\mathrm{id}, \sigma_{b}^{*} \alpha^{*}\left(\sigma_{\alpha(b)}^{-1}\right)^{*}\left(\sigma_{k}^{-1}\right)^{*} s\right) \otimes\left(\sigma_{k}, \operatorname{cst}_{y}\right) \\
\left.=\left(\mathrm{id}, \alpha^{*} \sigma_{\alpha(b)}^{*}\left(\sigma_{\alpha(b)}^{-1}\right)^{*} \sigma_{k}^{-1}\right)^{*} s\right) \otimes\left(\sigma_{k}, \operatorname{cst}_{y}\right) \\
=\left(\mathrm{id}, \alpha^{*}\left(\sigma_{k}^{-1}\right)^{*} s\right) \otimes\left(\sigma_{k}, \operatorname{cst}_{y}\right) .
\end{aligned}
$$

Compositions are the identity.

$$
\begin{aligned}
T_{y} W_{y}(\mathrm{id}, s) & =T_{y}\left(\left(\mathrm{id}, \alpha^{*}\left(\sigma_{k}^{-1}\right)^{*} s\right) \otimes\left(\sigma_{k}, \operatorname{cst}_{y}\right)\right) \\
& =\left(\mathrm{id}, \sigma_{k}^{*} \operatorname{ind}_{\alpha}\left(\alpha^{*}\left(\sigma_{k}^{-1}\right)^{*} s\right)\right) \\
& =(\mathrm{id}, s) .
\end{aligned}
$$

Given $(\sigma, t) \in R \Pi\left(K, \operatorname{ind}_{\alpha} X\right)\left(y, \operatorname{ind}_{\alpha}(x)\right)$ for $y \in \Pi\left(K, \operatorname{ind}_{\alpha} X\right)$ and $x \in \Pi(G, X)$ we know that $t_{1}=y$ and that $t_{0}=\operatorname{ind}_{\alpha}(x) \circ \sigma$, so $y \circ \sigma^{-1} \in G \times_{G} X$ and we have $\sigma_{k}=\sigma_{\alpha(b)} \sigma$ for an element $b \in G$. We calculate

$$
\begin{aligned}
W_{y} T_{y}((\mathrm{id}, v) \otimes(\sigma, t)) & =W_{y}\left(\left(\mathrm{id}, \sigma^{*}\left(\operatorname{ind}_{\alpha} v\right) t\right)\right) \\
& =\left(\mathrm{id}, \alpha^{*}\left(\sigma_{k}^{-1}\right)^{*}\left(\sigma^{*}\left(\operatorname{ind}_{\alpha} v\right) t\right)\right) \otimes\left(\sigma_{k}, \operatorname{cst}_{y}\right) \\
& =\left(\mathrm{id}, \alpha^{*} \sigma_{\alpha(b)}^{*}\left(\sigma_{k}^{-1}\right)^{*}\left(\sigma^{*}\left(\operatorname{ind}_{\alpha} v\right) t\right)\right) \otimes\left(\sigma, \operatorname{cst}_{y}\right) \\
& =\left((\mathrm{id}, v)\left(\mathrm{id}, \alpha^{*}\left(\sigma^{-1}\right)^{*} t\right)\right) \otimes\left(\sigma, \operatorname{cst}_{y}\right) \\
& =(\mathrm{id}, v) \otimes(\sigma, t) .
\end{aligned}
$$

Since the maps $T_{y}$ and $W_{y}$ are essentially given by composition of morphisms, functoriality is clear. So $T$ and $W$ are natural equivalences of $\Pi\left(K, \operatorname{ind}_{\alpha} X\right)$-modules. We see that the diagram

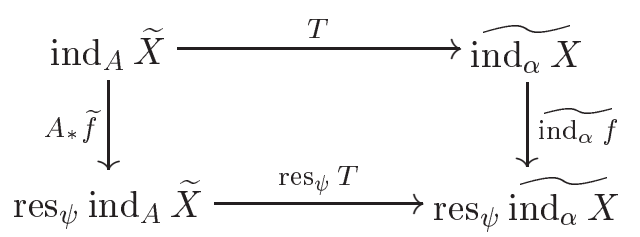


commutes since for an element (id, $v) \otimes(\sigma, w) \in \operatorname{ind}_{A} \widetilde{X}$ we have

$$
\begin{aligned}
& \left(\operatorname{res}_{\psi} T\right) \circ\left(A_{*} \widetilde{f}\right)((\mathrm{id}, v) \otimes(\sigma, w)) \\
& =\left(\operatorname{res}_{\psi} T\right)\left((\mathrm{id}, f(v)) \otimes\left(\sigma,\left(\operatorname{ind}_{\alpha} f\right)(w)\right) \otimes\left(\mathrm{id}, v_{y}\right)\right) \\
& =\left(\mathrm{id}, \sigma^{*}\left(\operatorname{ind}_{\alpha}(f(v))\right)\left(\operatorname{ind}_{\alpha} f\right)(w)\right) \otimes\left(\mathrm{id}, v_{y}\right) \\
& =\left(\mathrm{id},\left(\operatorname{ind}_{\alpha} f\right)\left(\sigma^{*}\left(\operatorname{ind}_{\alpha} v\right) w\right)\right) \otimes\left(\mathrm{id}, v_{y}\right) \\
& =\widetilde{\operatorname{ind}_{\alpha} f}\left(\left(\operatorname{id}, \sigma^{*}\left(\operatorname{ind}_{\alpha} v\right) w\right)\right) \\
& =\left(\widetilde{\operatorname{ind}_{\alpha} f}\right) \circ T((\operatorname{id}, v) \otimes(\sigma, w)) \text {. }
\end{aligned}
$$

where $\psi$ is induced by $\operatorname{ind}_{\alpha} f$ and $v_{y}$ is a path from $f(y)$ to $y$. So $T$ is a natural equivalence that maps $A_{*} \widetilde{f}$ to $\widetilde{\operatorname{ind}_{\alpha} f}$. We can now apply the cellular chain complex functor $C^{c}$ to obtain a natural equivalence $C^{c}(T): \operatorname{ind}_{A} C^{c}(\widetilde{X}) \rightarrow C^{c}\left(\widetilde{\operatorname{ind}_{\alpha} X}\right)$ which maps $A_{*} C^{c}(\widetilde{f})$ to $C^{c}\left(\widetilde{\operatorname{ind}_{\alpha} f}\right)$. So

$$
\left[A_{*} C^{c}(\widetilde{f})\right]=\left[C^{c}\left(\widetilde{\operatorname{ind}_{\alpha} f}\right)\right] \in K_{0}\left(\psi-\operatorname{end}_{\mathrm{ff} R \Pi\left(K, \operatorname{ind}_{\alpha} X\right)}\right),
$$

whence $\alpha_{*} u_{G}(X, f)=u_{K}\left(\operatorname{ind}_{\alpha} X, \operatorname{ind}_{\alpha} f\right)$.

Remark 3.5.3. Since $\left(U_{G}^{R}, u_{G}^{R}\right)$ is $G$-homotopy invariant and invariant under $G$-homotopy equivalence, we can define a group $U_{G}^{R}(X, f)$ and an element $u_{G}^{R}(X, f) \in U_{G}^{R}(X, f)$ for any continuous $G$-equivariant map $f: X \rightarrow X$, we need not restrict ourselves to cellular $G$-maps $f: X \rightarrow X$.

We start with the definition of $U_{G}^{R}(X, f)$. As in the case of a cellular map $f$, given a $G$-equivariant endomorphism $f: X \rightarrow X$, we define an abelian group $U_{G}^{R}(X, f):=K_{0}\left(\phi\right.$-end $\left.\operatorname{eff}_{\Pi(G, X)}\right)$, where $\phi:=\Pi(G, f)$. For a $G$-equivariant morphism $h:(X, f) \rightarrow(Y, g)$, i.e., a continuous map $h: X \rightarrow Y$ with $g h=h f$, we define a group homomorphism $U_{G}^{R}(h):=$ $K_{0}\left(\Pi(G, h)_{*}\right)$ as in Section 3.3. For a group homomorphism $\alpha: G \rightarrow K$ we set $U^{R}(\alpha):=K_{0}\left(\Pi\left(\operatorname{ind}_{\alpha}\right)_{*}\right)$ as in Section 3.4. We therefore have a family of functors $U_{G}^{R}$ not only on $\operatorname{End}\left(G-\mathcal{C} W_{\mathrm{fp}}\right)$, but on the category having all continuous maps instead of only cellular maps. As in the proof of Proposition 3.5.2, one checks $G$-homotopy invariance of the functor $U_{G}^{R}$ and the fact that a $G$-homotopy equivalence induces an isomorphism.

We now come to the definition of $u_{G}^{R}(X, f)$. When the map $f: X \rightarrow X$ is not cellular, it does not induce a map $C^{c}(\widetilde{f}): C^{c}(\widetilde{X}) \rightarrow \operatorname{res}_{\phi} C^{c}(\widetilde{X})$ of the cellular chain complex. We need to use an equivariant cellular approximation $f^{\prime}: X \rightarrow X$ of $f$. See Whitehead [Whi78, II.4] for cellular approximation in the non-equivariant case and tom Dieck [tD87, II.2] for the equivariant setting. There is a $G$-homotopy $F^{\prime}$ between $f^{\prime}$ and $f$, and the $G$-homotopy equivalence $i_{0}^{\prime}:\left(X, f^{\prime}\right) \rightarrow\left(X \times I,\left(F^{\prime}, \operatorname{id}_{I}\right)\right)$ induces an isomorphism $U_{G}^{R}\left(i_{0}^{\prime}\right): U_{G}^{R}\left(X, f^{\prime}\right) \rightarrow U_{G}^{R}\left(X \times I,\left(F^{\prime}, \operatorname{id}_{I}\right)\right)$. Analogously, we 
obtain an isomorphism $U_{G}^{R}\left(i_{1}^{\prime}\right): U_{G}^{R}(X, f) \rightarrow U_{G}^{R}\left(X \times I,\left(F^{\prime}, \mathrm{id}_{I}\right)\right)$. We set $u_{G}^{R}(X, f):=U_{G}^{R}\left(i_{1}^{\prime}\right)^{-1} U_{G}^{R}\left(i_{0}^{\prime}\right)\left(u_{G}^{R}\left(X, f^{\prime}\right)\right) \in U_{G}^{R}(X, f)$.

We need to check that the definition of $u_{G}^{R}(X, f)$ is independent of the choices of the cellular approximation $f^{\prime}$ and of the $G$-homotopy $F^{\prime}$. The isomorphism $U_{G}^{R}\left(i_{1}^{\prime}\right)^{-1} U_{G}^{R}\left(i_{0}^{\prime}\right): U_{G}^{R}\left(X, f^{\prime}\right) \rightarrow U_{G}^{R}(X, f)$ depends on the choices of $f^{\prime}$ and of $F^{\prime}$. Other choices $f^{\prime \prime}$ and $F^{\prime \prime}$ lead to another isomorphism $U_{G}^{R}\left(i_{1}^{\prime \prime}\right)^{-1} U_{G}^{R}\left(i_{0}^{\prime \prime}\right): U_{G}^{R}\left(X, f^{\prime \prime}\right) \rightarrow U_{G}^{R}(X, f)$. These isomorphisms differ by an isomorphism $U_{G}^{R}\left(i_{1}^{K}\right)^{-1} U_{G}^{R}\left(i_{0}^{K}\right): U_{G}^{R}\left(X, f^{\prime}\right) \rightarrow U_{G}^{R}\left(X, f^{\prime \prime}\right)$ induced by the $G$-homotopy $K:=F^{\prime} \cup_{f}\left(-F^{\prime \prime}\right)$ from $f^{\prime}$ to $f^{\prime \prime}$, i.e, we have $U_{G}^{R}\left(i_{1}^{K}\right)^{-1} U_{G}^{R}\left(i_{0}^{K}\right)=\left(U_{G}^{R}\left(i_{1}^{\prime \prime}\right)^{-1} U_{G}^{R}\left(i_{0}^{\prime \prime}\right)\right)^{-1}\left(U_{G}^{R}\left(i_{1}^{\prime}\right)^{-1} U_{G}^{R}\left(i_{0}^{\prime}\right)\right)$.

Take $L: X \times I \rightarrow X$ to be a cellular approximation of $K$. Then $K$ is $G$-homotopic to the cellular $G$-map $L$ relative $X \times \partial I$, so there is a $G$-map $H: X \times I \times I \rightarrow X$ with $\left.H\right|_{X \times I \times\{0\}}=K,\left.H\right|_{X \times I \times\{1\}}=L,\left.H\right|_{X \times\{0\} \times\{s\}}=f^{\prime}$ for all $s \in I$ and $\left.H\right|_{X \times\{1\} \times\{s\}}=f^{\prime \prime}$ for all $s \in I$. We have a commutative diagram

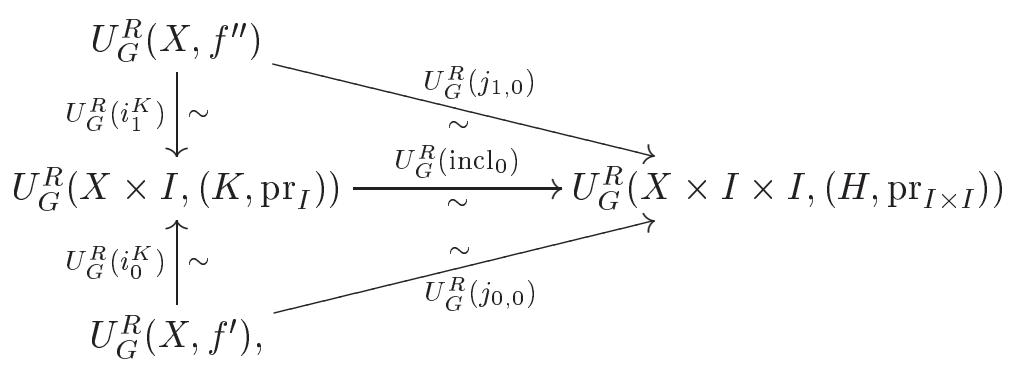

whence $U_{G}^{R}\left(i_{1}^{K}\right)^{-1} U_{G}^{R}\left(i_{0}^{K}\right)=U_{G}^{R}\left(j_{1,0}\right)^{-1} U_{G}^{R}\left(j_{0,0}\right)$. Analogously, we obtain

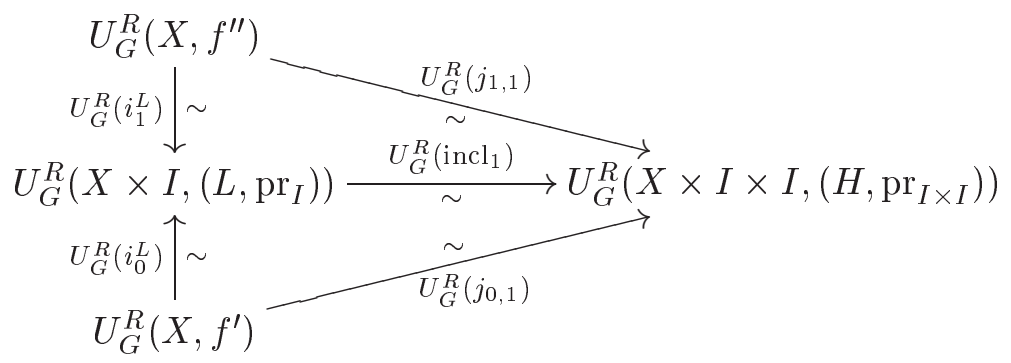

and thus $U_{G}^{R}\left(i_{1}^{L}\right)^{-1} U_{G}^{R}\left(i_{0}^{L}\right)=U_{G}^{R}\left(j_{1,1}\right)^{-1} U_{G}^{R}\left(j_{0,1}\right)$.

We know that $\operatorname{id}_{X} \times\{0\} \times \operatorname{id}_{I}: X \times I \rightarrow X \times I \times I$ gives a $G$-homotopy between $j_{0,0}$ and $j_{0,1}$ as maps from $\left(X, f^{\prime}\right)$ to $\left(X \times I \times I,\left(H, \mathrm{pr}_{I \times I}\right)\right)$ since $\left.H\right|_{X \times\{0\} \times\{s\}}=f^{\prime}$ for all $s \in I$. Analogously, we have a $G$-homotopy between $j_{1,0}$ and $j_{1,1}$ as maps from $\left(X, f^{\prime \prime}\right)$ to $\left(X \times I \times I,\left(H, \operatorname{pr}_{I \times I}\right)\right)$. By $G$-homotopy invariance we conclude that

$$
\begin{aligned}
U_{G}^{R}\left(i_{1}^{K}\right)^{-1} U_{G}^{R}\left(i_{0}^{K}\right) & =U_{G}^{R}\left(j_{1,0}\right)^{-1} U_{G}^{R}\left(j_{0,0}\right)=U_{G}^{R}\left(j_{1,1}\right)^{-1} U_{G}^{R}\left(j_{0,1}\right) \\
& =U_{G}^{R}\left(i_{1}^{L}\right)^{-1} U_{G}^{R}\left(i_{0}^{L}\right) .
\end{aligned}
$$


Since invariance under $G$-homotopy equivalence is known for the cellular case, $U_{G}^{R}\left(i_{1}^{L}\right)^{-1} U_{G}^{R}\left(i_{0}^{L}\right)$ maps the invariant $u_{G}^{R}\left(X, f^{\prime}\right)$ to $u_{G}^{R}\left(X, f^{\prime \prime}\right)$. So $U_{G}^{R}\left(i_{1}^{K}\right)^{-1} U_{G}^{R}\left(i_{0}^{K}\right)=U_{G}^{R}\left(i_{1}^{L}\right)^{-1} U_{G}^{R}\left(i_{0}^{L}\right)$ also does, and we have

$$
\begin{aligned}
& U_{G}^{R}\left(i_{1}^{\prime \prime}\right)^{-1} U_{G}^{R}\left(i_{0}^{\prime \prime}\right)\left(u_{G}^{R}\left(X, f^{\prime \prime}\right)\right) \\
& =U_{G}^{R}\left(i_{1}^{\prime \prime}\right)^{-1} U_{G}^{R}\left(i_{0}^{\prime \prime}\right)\left(U_{G}^{R}\left(i_{1}^{K}\right)^{-1} U_{G}^{R}\left(i_{0}^{K}\right)\left(u_{G}^{R}\left(X, f^{\prime}\right)\right)\right) \\
& =U_{G}^{R}\left(i_{1}^{\prime}\right)^{-1} U_{G}^{R}\left(i_{0}^{\prime}\right)\left(u_{G}^{R}\left(X, f^{\prime}\right)\right) .
\end{aligned}
$$

Hence the element $u_{G}^{R}(X, f):=U_{G}^{R}\left(i_{1}^{\prime}\right)^{-1} U_{G}^{R}\left(i_{0}^{\prime}\right)\left(u_{G}^{R}\left(X, f^{\prime}\right)\right) \in U_{G}^{R}(X, f)$ is independent of the choice of the cellular approximation $f^{\prime}$ and the $G$ homotopy $F^{\prime}$. 


\section{Chapter 4}

\section{Universality}

In this chapter, we arrive at the first climax of this thesis. We show that the invariant $\left(U_{G}^{\mathbb{Z}}, u_{G}^{\mathbb{Z}}\right)$ constructed in Chapter 3 has a universal initial property among all functorial equivariant Lefschetz invariants. It will be called the universal functorial equivariant Lefschetz invariant. This theorem, Theorem 4.1.2, explains the theoretical importance of the invariant $\left(U_{G}^{\mathbb{Z}}, u_{G}^{\mathbb{Z}}\right)$ and justifies our interest in it. This chapter is the equivariant generalization of [Lüc99, Chapter 4].

In the first section, we state the results and introduce the notation necessary in the proof. We explain retractive $G$-spaces, generalizations of pointed spaces. A $G$-space $X$ plays the role of the basepoint. One can define cones and suspensions. In Lemma 4.1.3 we prove the key ingredient of the proof of Theorem 4.1.2, namely a way to get back from algebra to geometry. There the retractive $G$-spaces come into play.

\subsection{Notations and Results}

We start by stating what we mean by a universal functorial Lefschetz invariant. In Definition 3.5.1 we defined functorial equivariant Lefschetz invariants, and we now state what it means to be universal among all of those.

Definition 4.1.1. A functorial equivariant Lefschetz invariant $\left(U_{G}, u_{G}\right)$ is called universal if for any functorial equivariant Lefschetz invariant $\left(\Theta_{G}, \theta_{G}\right)$ there is precisely one family of natural transformations $\tau_{G}: U_{G} \rightarrow \Theta_{G}$ such that $\tau_{G(X, f)}: U_{G}(X, f) \rightarrow \Theta_{G}(X, f)$ sends $u_{G}(X, f)$ to $\theta_{G}(X, f)$ for any object $(X, f)$ in $\operatorname{End}\left(G-\mathcal{C} W_{\mathrm{fp}}\right)$, for any discrete group $G$, and such that the equality $\tau_{K} \circ U(\alpha)=\Theta(\alpha) \circ \tau_{G}$ holds for inclusions $\alpha: G \rightarrow K$.

The goal of this chapter is to prove the following theorem.

Theorem 4.1.2. The pair $\left(U_{G}^{\mathbb{Z}}, u_{G}^{\mathbb{Z}}\right)$ is the universal functorial equivariant Lefschetz invariant on the family of categories $\operatorname{End}\left(G-\mathcal{C} W_{\mathrm{fp}}\right)$ for discrete groups $G$. 
Before we start the proof, we introduce the necessary notation, generalizing Lück [Lüc99, Chapter 4]. We define retractive $G$-spaces over a given $G$-space $X$, which for $X$ the one-point space are just pointed $G$-spaces. Then we define analogs of the cone and the suspension. We also define the analog of pointed homotopy classes of maps, namely those that induce a given map $f$ on $X$.

Let $X$ be a $G$-space. A retractive $G$-space $Y$ over $X$ is a triple $Y=$ $(Y, i, r)$ which consists of a $G$-space $Y$, a $G$-cofibration $i: X \rightarrow Y$ and a $G$-map $r: Y \rightarrow X$ satisfying $r \circ i=\operatorname{id}_{X}$. We often identify $X$ with $i(X)$. Given a retractive $G$-space $Y$ over $X$, define retractive $G$-spaces $Y \times_{X}[0,1]$ and $C_{X} Y$ to be the pushouts

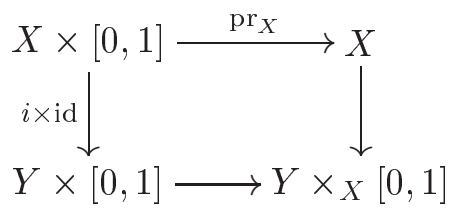

and

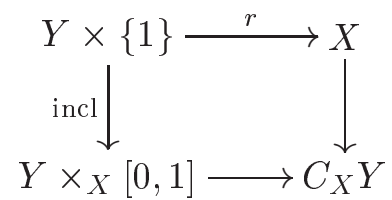

respectively, where $[0,1]$ is endowed with the trivial $G$-action. The inclusions of $X$ into $Y \times_{X}[0,1]$ and into $C_{X} Y$ are the right vertical maps, the retractions of $Y \times_{X}[0,1]$ onto $X$ and the retraction $\widehat{r}: C_{X} Y \rightarrow X$ onto $X$ are induced by the retraction $r: Y \rightarrow X$ by the pushout property. Define the retractive $G$-space $\Sigma_{X} Y$ to be the pushout

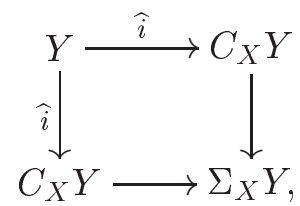

where $\widehat{i}: Y \rightarrow C_{X} Y$ is the inclusion induced by the inclusion $Y \times\{0\} \rightarrow$ $Y \times[0,1]$. The retraction is induced by $\widehat{r}$ by the pushout property. The composition $\hat{i} \circ i: X \rightarrow C_{X} Y$ is a $G$-homotopy equivalence relative $X$ with the retraction of $C_{X} Y$ onto $X$ as $G$-homotopy inverse relative $X$.

Given two retractive spaces $Y$ and $Z$ over $X$ and a $G$-endomorphism $f: X \rightarrow X$, define $\left[\left(C_{X} Y, Y\right),\left(C_{X} Z, Z\right)\right]_{f}^{G}$ to be the set of $G$-homotopy classes relative $X$ of maps of pairs $(\widehat{g}, g):\left(C_{X} Y, Y\right) \rightarrow\left(C_{X} Z, Z\right)$ that induce the given endomorphism $f$ on $X$, i.e., such that $g \circ i_{Y}=i_{Z} \circ f$. (Homotopy class relative $X$ means that the relevant homotopies are stationary on $X$.)

Let $\widetilde{X}$ be the universal covering space of $X$, a $\Pi(G, X)$-space, and let $\widetilde{Y}$ be the universal covering space of $Y$, a $\Pi(G, Y)$-space. Then $\operatorname{res}_{\Pi\left(G, i_{Y}\right)} \tilde{Y}$ is 
a $\Pi(G, X)$-space. We define the $\mathbb{Z} \Pi(G, X)$-chain complex $C^{c}(\widetilde{Y}, \widetilde{X})$ by

$$
C^{c}(\widetilde{Y}, \widetilde{X}):=\operatorname{coker}\left(C^{c}(\widetilde{X}) \stackrel{C^{c}(\widetilde{i})}{\longrightarrow} \operatorname{res}_{\Pi\left(G, i_{Y}\right)} C^{c}(\widetilde{Y})\right) .
$$

We call a retractive $G$-space $Y$ over $X$ a $d$-extension if $Y$ is obtained from $X$ by attaching finitely many cells in dimension $d$. If $Y$ is a $d$-extension of $X$ and $d \geq 2$, then we have the short exact sequence

$$
0 \rightarrow C^{c}(\widetilde{X}) \stackrel{C^{c}(\widetilde{i})}{\longrightarrow} \operatorname{res}_{\Pi\left(G, i_{Y}\right)} C^{c}(\widetilde{Y}) \rightarrow C^{c}(\widetilde{Y}, \widetilde{X}) \rightarrow 0
$$

where $C^{c}(\widetilde{X})$ and $\operatorname{res}_{\Pi\left(G, i_{Y}\right)} C^{c}(\widetilde{Y})$ are finite free $\mathbb{Z} \Pi(G, X)$-chain complexes equipped with the cellular equivalence class of bases [Lüc89, Definition 13.3 and Example 9.18]. Since the above sequence is based split exact, $C^{c}(\widetilde{Y}, \widetilde{X})$ is a chain complex concentrated in degree $d$, and $C_{d}^{c}(\widetilde{Y}, \widetilde{X})$ is a finite free $\mathbb{Z} \Pi(G, X)$-module with an equivalence class of bases given by the cells of $Y \backslash X$ [Lüc89, Example 9.18].

Note that if $Y$ is a $d$-extension of $X$ and $d \geq 2$, then the functor $\Pi\left(G, i_{Y}\right): \Pi(G, X) \rightarrow \Pi(G, Y)$ is an equivalence of categories with inverse $\Pi\left(G, r_{Y}\right)$. This holds because the retraction $r_{Y}: Y \rightarrow X$ exists. In general, if there is only an inclusion given, it is only true for $d \geq 3$. The fact that $\Pi\left(G, i_{Y}\right)$ is an equivalence of categories implies that $\operatorname{res}_{\Pi\left(G, i_{Y}\right)} C^{c}(\widetilde{Y})$ is a finite free $\mathbb{Z} \Pi(G, X)$-module, so $\operatorname{res}_{\Pi\left(G, i_{Y}\right)} C^{c}(\widetilde{g}) \in \phi$-end $\operatorname{eff}_{\mathrm{f}} \mathbb{Z}(G, X)$.

Remember that we have a canonical lift $\widetilde{f}: \widetilde{X} \rightarrow \widetilde{X} \circ \phi$ of $f: X \rightarrow X$ given by the lifts $\widetilde{\left.f\right|_{X^{H}(x)}}: \widetilde{X^{H}(x)} \rightarrow \widehat{X^{H}(f(x))}$ with $\left[\operatorname{id}_{x}\right] \mapsto\left[\operatorname{id}_{f(x)}\right]$. For any map $g: Y \rightarrow Z$ of $d$-extensions of $X$, for $d \geq 2$, such that $g \circ i_{Y}=i_{Z} \circ f$ we obtain a lift $\widetilde{g}: \operatorname{res}_{\Pi\left(G, i_{Y}\right)} \widetilde{Y} \rightarrow \operatorname{res}_{\Pi\left(G, i_{Z}\right)} \widetilde{Z} \circ \phi$ uniquely determined by the fact that it induces $\widetilde{f}$ on $\widetilde{X}$. The map $\widetilde{g}$ is a map of $\Pi(G, X)$-spaces. If we have a $G$-endomorphism $f: X \rightarrow X$ and a $G$-endomorphism $g: Y \rightarrow Y$ extending $f$, then

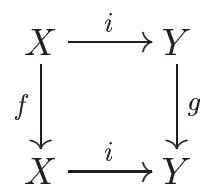

commutes, so

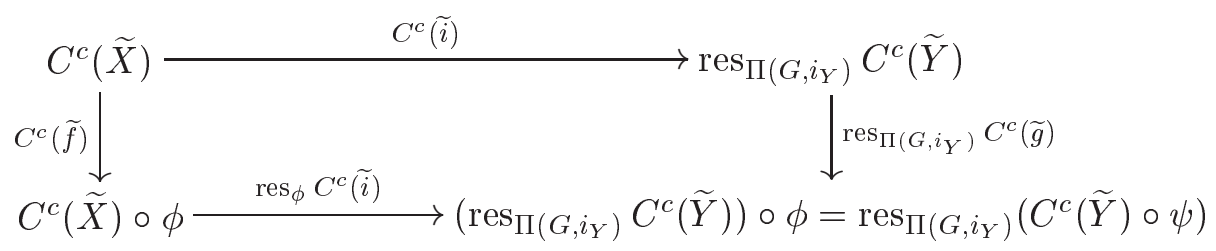

commutes as well, and we have an induced map between the cokernels of the horizontal maps, $C^{c}(\widetilde{g}, \widetilde{f}): C^{c}(\widetilde{Y}, \widetilde{X}) \rightarrow C^{c}(\widetilde{Y}, \widetilde{X}) \circ \phi$, which is a map of $\Pi(G, X)$-chain complexes. 
The next lemma contains the decisive bridge between geometry and algebra, in analogy to Lück [Lüc99, Lemma 4.2].

Lemma 4.1.3. Let $Y$ and $Z$ be $d$-extensions of the $G$-space $X$ with $d \geq 2$. Then the map

$$
\begin{aligned}
\eta:\left[\left(C_{X} Y, Y\right),\left(C_{X} Z, Z\right)\right]_{f}^{G} & \rightarrow \operatorname{Mor}_{\mathbb{Z} \Pi(G, X)}\left(C_{d}^{c}(\widetilde{Y}, \widetilde{X}), C_{d}^{c}(\widetilde{Z}, \widetilde{X}) \circ \phi\right) \\
{[(\widehat{g}, g)] } & \mapsto C_{d}^{c}(\widetilde{g}, \widetilde{f})
\end{aligned}
$$

is bijective.

Proof. Choose a $G$-pushout

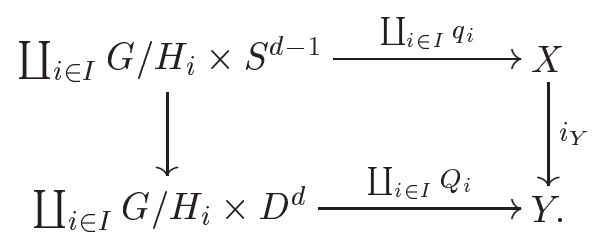

Define a $G$-map $p_{i}: G / H_{i} \times S^{d} \rightarrow Y$ by setting $\left.p_{i}\right|_{G / H_{i} \times S_{+}^{d}}:=Q_{i}$ and $\left.p_{i}\right|_{G / H_{i} \times S_{-}^{d}}:=r \circ Q_{i}$. Then we can see $C_{X} Y$ as the pushout

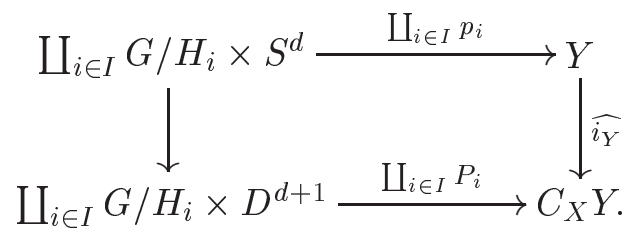

Hence a $G$-map $(\widehat{g}, g):\left(C_{X} Y, Y\right) \rightarrow\left(C_{X} Z, Z\right)$ with $\left.g\right|_{X}=f$ is uniquely determined by its compositions $(\widehat{g}, g) \circ\left(P_{i}, p_{i}\right)$, and any collection of $G$-maps $\left(\widehat{k_{i}}, k_{i}\right):\left(G / H_{i} \times D^{d+1}, G / H_{i} \times S^{d}\right) \rightarrow\left(C_{X} Z, Z\right)$ with $\left.k_{i}\right|_{G / H_{i} \times S_{-}^{d}}=f \circ r \circ Q_{i}$ determines uniquely such a map $(\widehat{g}, g)$ with $(\widehat{g}, g) \circ\left(P_{i}, p_{i}\right)=\left(\widehat{k_{i}}, k_{i}\right)$. Hence the map

$$
\begin{aligned}
\mu: & {\left[\left(C_{X} Y, Y\right),\left(C_{X} Z, Z\right)\right]_{f}^{G} } \\
& \rightarrow \prod_{i \in I}\left[G / H_{i} \times\left(D^{d+1}, S^{d}\right),\left(C_{X} Z, Z\right)\right]_{f \circ r \circ Q_{i}: G / H_{i} \times S_{-}^{d} \rightarrow X}^{G}
\end{aligned}
$$

sending $[(\widehat{g}, g)]$ to $\left(\left[(\widehat{g}, g) \circ\left(P_{i}, p_{i}\right)\right]\right)_{i \in I}$ is a bijection. The following forgetful map is bijective for all $i \in I$ :

$$
\begin{aligned}
\nu_{i}: & {\left[G / H_{i} \times\left(D^{d+1}, S^{d}\right),\left(C_{X} Z, Z\right)\right]_{f \circ r \circ Q_{i}: G / H_{i} \times S_{-}^{d} \rightarrow X}^{G} } \\
& \stackrel{\sim}{\rightarrow}\left[G / H_{i} \times\left(D^{d+1}, S^{d}, s\right),\left(C_{X} Z, Z, f\left(x_{i}\right)\right)\right]^{G} \\
& \simeq \pi_{d+1}\left(\left(C_{X} Z\right)^{H_{i}}\left(f\left(x_{i}\right)\right), Z^{H_{i}}\left(f\left(x_{i}\right)\right), f\left(x_{i}\right)\right),
\end{aligned}
$$


where $s=(0, \ldots, 0,-1) \in S^{d-1} \subseteq D^{d} \cong S_{-}^{d} \subseteq S^{d}$ is a fixed base point and $x_{i}:=q_{i}(-, s): G / H_{i} \rightarrow X \in \Pi(G, X)$. Here $f\left(x_{i}\right)$ means either $f \circ x_{i}\left(G / H_{i}\right)$ or $f\left(x_{i}\left(1 H_{i}\right)\right)$, depending on the context. The forgetful map $\nu_{i}$ forgets that the map has to look like $f \circ r \circ Q_{i}$ on $G / H_{i} \times S_{-}^{d}$ and remembers only that it is $f \circ x_{i}$ on $G / H_{i} \times\{s\}$. The map $\nu_{i}$ is bijective since the inclusion $G / H_{i} \times\{s\} \rightarrow G / H_{i} \times S_{-}^{d}$ is a $G$-homotopy equivalence and the inclusions $\{s\} \rightarrow S_{-}^{d} \rightarrow S^{d} \rightarrow D^{d+1}$ are cofibrations.

The inclusion $X \rightarrow C_{X} Z$ is a $G$-homotopy equivalence, so for all $H_{i} \leq G$ the inclusion $X^{H_{i}}\left(f\left(x_{i}\right)\right) \rightarrow\left(C_{X} Z\right)^{H_{i}}\left(f\left(x_{i}\right)\right)$ is a $G$-homotopy equivalence. Thus we know that $\pi_{d+1}\left(\left(C_{X} Z\right)^{H_{i}}\left(f\left(x_{i}\right)\right), X^{H_{i}}\left(f\left(x_{i}\right)\right), f\left(x_{i}\right)\right) \cong 0$ and that $\pi_{d}\left(\left(C_{X} Z\right)^{H_{i}}\left(f\left(x_{i}\right)\right), X^{H_{i}}\left(f\left(x_{i}\right)\right), f\left(x_{i}\right)\right) \cong 0$, and the boundary map is an isomorphism

$$
\begin{aligned}
& \partial: \pi_{d+1}\left(\left(C_{X} Z\right)^{H_{i}}\left(f\left(x_{i}\right)\right), Z^{H_{i}}\left(f\left(x_{i}\right)\right), f\left(x_{i}\right)\right) \cong\left[G / H_{i} \times\left(D^{d+1}, S^{d}, s\right),\left(C_{X} Z, Z, f\left(x_{i}\right)\right)\right]^{G} \\
& \stackrel{\sim}{\longrightarrow} \pi_{d}\left(Z^{H_{i}}\left(f\left(x_{i}\right)\right), X^{H_{i}}\left(f\left(x_{i}\right)\right), f\left(x_{i}\right)\right) \\
& \cong\left[G / H_{i} \times\left(D^{d}, S^{d-1}, s\right),\left(Z, X, f\left(x_{i}\right)\right)\right]^{G}, \\
& {[(\widehat{h}, h)] \quad \mapsto \quad\left[h \circ\left(\operatorname{id}_{G / H_{i}} \times \operatorname{pr}_{D^{d} \rightarrow S^{d}}\right), s \circ\left(\operatorname{id}_{G / H_{i}} \times \operatorname{pr}_{S^{d-1} \rightarrow s}\right)\right] . }
\end{aligned}
$$

So we have an isomorphism

$$
\begin{aligned}
\left(\prod_{c \in I} \partial\right)\left(\prod_{i \in I} \nu_{i}\right) \mu: & \\
{\left[\left(C_{X} Y, Y\right),\left(C_{X} Z, Z\right)\right]_{f}^{G} } & \stackrel{\sim}{\rightarrow} \prod_{i \in I} \pi_{d}\left(Z^{H_{i}}\left(f\left(x_{i}\right)\right), X^{H_{i}}\left(f\left(x_{i}\right)\right), f\left(x_{i}\right)\right) \\
{[(\widehat{g}, g)] } & \mapsto\left(\left[g \circ Q_{i}\left(1 H_{i},-\right), f \circ q_{i}\left(1 H_{i},-\right)\right]\right)_{i \in I} .
\end{aligned}
$$

We know that

$$
\begin{aligned}
& \left.\left.\pi_{d}\left(Z^{H_{i}}\left(f\left(x_{i}\right)\right), X^{H_{i}}\left(f\left(x_{i}\right)\right), f\left(x_{i}\right)\right) \stackrel{p \sim}{\longleftarrow} \pi_{d}\left(\widetilde{Z^{H_{i}}\left(f\left(x_{i}\right)\right.}\right), \widetilde{X^{H_{i}}\left(f\left(x_{i}\right)\right.}\right), \widetilde{f\left(x_{i}\right)}\right) \\
& \left.\left.\stackrel{h \sim}{\longrightarrow} H_{d}\left(Z^{H_{i}\left(f\left(x_{i}\right)\right.}\right), \widehat{X^{H_{i}}\left(f\left(x_{i}\right)\right)}\right)=C_{d}^{c}\left(\widetilde{Z^{H_{i}}\left(f\left(x_{i}\right)\right.}\right), \widehat{X^{H_{i}}\left(f\left(x_{i}\right)\right)}\right) \\
& =\left(C_{d}^{c}(\widetilde{Z}, \widetilde{X}) \circ \phi\right)\left(x_{i}\right) \text {. }
\end{aligned}
$$

Here $p=\pi_{d}\left(\operatorname{pr}, \widetilde{f\left(x_{i}\right)}\right)$, the map induced by the projections of the covering spaces $Z^{H_{i}\left(f\left(x_{i}\right)\right)}$ and $X^{H_{i}\left(f\left(x_{i}\right)\right)}$ onto the respective space, is an isomorphism since the pair $\left(Z^{H_{i}}\left(f\left(x_{i}\right)\right), X^{H_{i}}\left(f\left(x_{i}\right)\right)\right)$ is at least 2-connected. The map $h$ denotes the Hurewicz map, which is an isomorphism since it is in the first non-vanishing degree of homotopy/homology.

We know that $C_{d}^{c}(\widetilde{Y}, \widetilde{X})$ is a finite free $\mathbb{Z} \Pi(G, X)$-module with an equivalence class of bases given by the cells of $Y \backslash X$, and that we can choose a basis using the characteristic maps $\left(Q_{i}, q_{i}\right)$ such that

$$
C_{d}^{c}(\widetilde{Y}, \widetilde{X}) \cong \bigoplus_{i \in I} \mathbb{Z} \Pi(G, X)\left(?, q_{i}(-, s)\right)
$$


Once again we set $x_{i}:=q_{i}(-, s): G / H_{i} \rightarrow X \in \Pi(G, X)$. We obtain:

$$
\begin{aligned}
& {\left[\left(C_{X} Y, Y\right),\left(C_{X} Z, Z\right)\right]_{f}^{G}} \\
& \cong \prod_{i \in I} \pi_{d}\left(Z^{H_{i}}\left(f\left(x_{i}\right)\right), X^{H_{i}}\left(f\left(x_{i}\right)\right), f\left(x_{i}\right)\right) \\
& \cong \prod_{i \in I}\left(C_{d}^{c}(\widetilde{Z}, \widetilde{X}) \circ \phi\right)\left(x_{i}\right) \\
& \cong \operatorname{Mor}_{\mathbb{Z} \Pi(G, X)}\left(\bigoplus_{i \in I} \mathbb{Z} \Pi(G, X)\left(?, x_{i}\right), C_{d}^{c}(\widetilde{Z}, \widetilde{X}) \circ \phi\right) \\
& \cong \operatorname{Mor}_{\mathbb{Z} \Pi(G, X)}\left(C_{d}^{c}(\widetilde{Y}, \widetilde{X}), C_{d}^{c}(\widetilde{Z}, \widetilde{X}) \circ \phi\right) .
\end{aligned}
$$

Under this isomorphism,

$$
\begin{aligned}
& {[(\widehat{g}, g)]} \\
& \quad \mapsto \quad\left(\left[g \circ Q_{i}\left(1 H_{i},-\right), f \circ q_{i}\left(1 H_{i},-\right)\right]\right)_{i \in I} \\
& \quad \mapsto \quad\left((\widetilde{g}, \widetilde{f})\left(\widetilde{Q_{i}}\left(1 H_{i},\left[D^{d}\right]\right), \widetilde{q_{i}}\left(1 H_{i},\left[S^{d}\right]\right)\right)\right)_{i \in I}=\left(C_{d}^{c}(\widetilde{g}, \widetilde{f})\left(x_{i}\right)\right)_{i \in I} \\
& \quad \mapsto \quad\left(\sum_{i \in I} v_{i} \mapsto \sum_{i \in I} v_{i}^{*} C_{d}^{c}(\widetilde{g}, \widetilde{f})\left(x_{i}\right)\right) \\
& \quad \mapsto C_{d}^{c}(\widetilde{g}, \widetilde{f}) .
\end{aligned}
$$

Here a priori the choice of bases plays a role, but one can check that with another choice of bases one obtains the same element $C_{d}^{c}(\widetilde{g}, \widetilde{f})$ in $\operatorname{Mor}_{\mathbb{Z} \Pi(G, X)}\left(C_{d}^{c}(\widetilde{Y}, \widetilde{X}), C_{d}^{c}(\widetilde{Z}, \widetilde{X}) \circ \phi\right)$. The isomorphism constructed above is the desired isomorphism

$$
\begin{aligned}
\eta:\left[\left(C_{X} Y, Y\right),\left(C_{X} Z, Z\right)\right]_{f}^{G} & \stackrel{\sim}{\rightarrow} \operatorname{Mor}_{\mathbb{Z} \Pi(G, X)}\left(C_{d}^{c}(\widetilde{Y}, \widetilde{X}), C_{d}^{c}(\widetilde{Z}, \widetilde{X}) \circ \phi\right) \\
{[(\widehat{g}, g)] } & \mapsto C_{d}^{c}(\widetilde{g}, \widetilde{f}) .
\end{aligned}
$$

Remark 4.1.4. The isomorphism $\eta$ is compatible with compositions. For $[(\widehat{g}, g)] \times[(\widehat{h}, h)] \in\left[\left(C_{X} Y, Y\right),\left(C_{X} Z, Z\right)\right]_{f}^{G} \times\left[\left(C_{X} Z, Z\right),\left(C_{X} W, W\right)\right]_{f^{\prime}}^{G}$ we have

$$
\begin{aligned}
\eta([(\widehat{h} \widehat{g}, h g)]) & =C_{d}^{c}\left(\widetilde{h g}, \widetilde{f^{\prime} f}\right) \\
& =\operatorname{res}_{\phi}\left(C_{d}^{c}\left(\widetilde{h}, \widetilde{f^{\prime}}\right)\right) \circ C_{d}^{c}(\widetilde{g}, \widetilde{f}) \\
& =\operatorname{res}_{\phi} \eta([(\widehat{g}, g)]) \circ \eta([(\widehat{h}, h)]),
\end{aligned}
$$

and this is the composition of $\eta([(\widehat{g}, g)])$ and $\eta([(\widehat{h}, h)])$.

Remark 4.1.5. The use of retractive spaces suggests connections to algebraic $K$-theory of spaces developed by Waldhausen [Wal85]. It would be interesting to try to generalize results about the Euler characteristic in this setting to the Lefschetz number and to extend the parametrized family approach [DWW03]. 


\subsection{Proof of the Universal Property}

Now we are ready for the proof of Theorem 4.1.2.

Proof (of Theorem 4.1.2). We have already shown that the pair $\left(U_{G}^{\mathbb{Z}}, u_{G}^{\mathbb{Z}}\right)$ defined in Chapter 3 is a functorial equivariant Lefschetz invariant. It remains to prove universality. For every functorial equivariant Lefschetz invariant $\left(\Theta_{G}, \theta_{G}\right)$ we need to find a natural transformation $\tau_{G}: U_{G}^{\mathbb{Z}} \rightarrow \Theta_{G}$ such that $u_{G}^{R}(X, f)$ maps to $\theta_{G}(X, f)$ for all $(X, f)$ in $\operatorname{End}\left(G-\mathcal{C} W_{\mathrm{fp}}\right)$, for discrete groups $G$, and such that $\tau_{K} \circ U(\alpha)=\Theta(\alpha) \circ \tau_{G}$ for inclusions $\alpha: G \rightarrow K$.

We define $\tau$ by translating the algebraic data encoded by $U_{G}^{\mathbb{Z}}$ back into geometric information using Lemma 4.1.3. We will omit the index $G$ most of the time if no confusion can occur. We proceed in eight steps.

We start by defining a map $\tau_{Y}:\left[\left(C_{X} Y, Y\right),\left(C_{X} Y, Y\right)\right]_{f}^{G} \rightarrow \Theta(X, f)$ for any $d$-extension $Y$ of $X$, with $d \geq 1$. In the next step we define a map $\tau_{d}: U_{G}^{\mathbb{Z}}(X, f) \rightarrow \Theta_{G}(X, f)$ for $d \geq 2$. Then we check that $\tau_{d}$ is well-defined and that $\tau_{d}$ is independent of the choice of the integer $d \geq 2$. Having defined this $\operatorname{map} \tau_{G(X, f)}: U_{G}^{\mathbb{Z}}(X, f) \rightarrow \Theta_{G}(X, f)$ for $(X, f)$ in $\operatorname{End}\left(G-\mathcal{C} W_{\mathrm{fp}}\right)$, for discrete groups $G$, we check that $\tau$ is actually a natural transformation from $U_{G}^{\mathbb{Z}}$ to $\Theta_{G}$. Next we show that the natural transformation $\tau: U_{G}^{\mathbb{Z}} \rightarrow \Theta_{G}$ maps $u_{G}^{\mathbb{Z}}(X, f) \in U_{G}^{\mathbb{Z}}(X, f)$ to $\theta_{G}(X, f) \in \Theta_{G}(X, f)$ for all $(X, f)$ in $\operatorname{End}\left(G-\mathcal{C} W_{\mathrm{fp}}\right)$, for discrete groups $G$. Then we prove compatibility with the induction structure. We finally prove uniqueness of the natural transformation $\tau$.

Step 1: We define

$$
\begin{aligned}
\tau_{Y}:\left[\left(C_{X} Y, Y\right),\left(C_{X} Y, Y\right)\right]_{f}^{G} & \rightarrow \Theta(X, f) \\
{[(\widehat{g}, g)] } & \mapsto \Theta(\widehat{i} \circ i)^{-1} \Theta(\widehat{i})(\theta(g))
\end{aligned}
$$

for any $d$-extension $Y$ of $X$, with $d \geq 1$. Since we have a map $\widehat{g}: C_{X} Y \rightarrow$ $C_{X} Y$ extending $g$, the maps $\widehat{i}$ and $\hat{i} \circ i$ are indeed maps in our endomorphism category: The diagrams

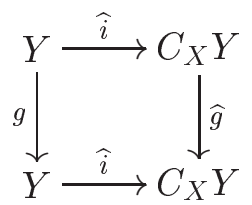

and

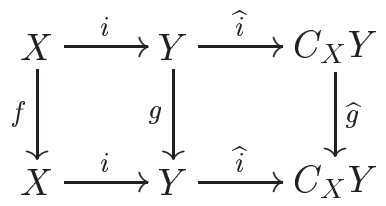

commute. Recall that $i$ and $\hat{i}$ are inclusions and $\hat{i} \circ i$ is a $G$-homotopy equivalence, so $\Theta(\hat{i} \circ i)$ is bijective by invariance under $G$-homotopy equivalence. 
The maps $g$ and $\widehat{g}$ are only defined up to $G$-homotopy (relative $X$ ), but because of the $G$-homotopy invariance of $(\Theta, \theta)$ this does not play a role: Let $(\widehat{G}, G)$ be a $G$-homotopy relative $X$ between $\left(\widehat{g_{0}}, g_{0}\right)$ and $\left(\widehat{g_{1}}, g_{1}\right)$. Let $l_{0}$ and $l_{1}$ be the inclusions of $X$ as $X \times\{0\}$ and $X \times\{1\}$ respectively into $X \times I$, and analogously for $Y$ and $C_{X} Y$. Consider the commutative diagram

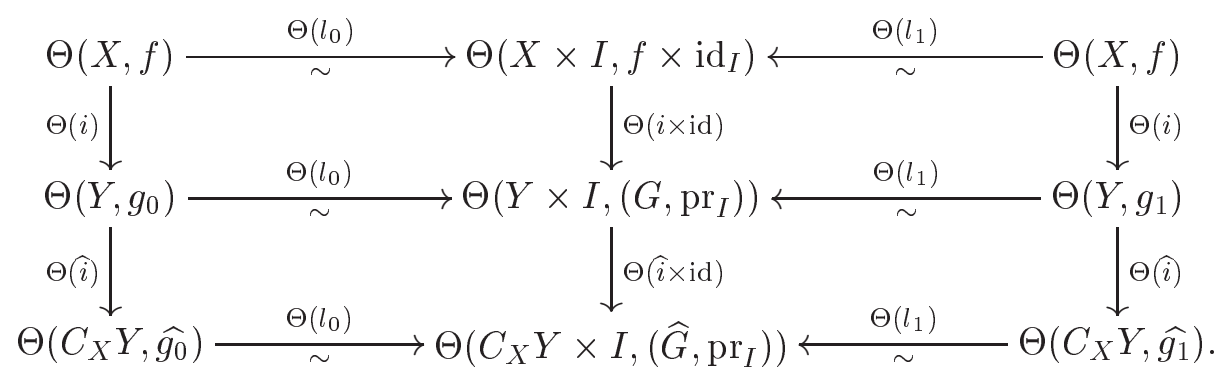

The horizontal maps are bijective because they are $G$-homotopy equivalences, and they map $\theta(f)$ to $\theta\left(f \times \operatorname{id}_{I}\right)$, both $\theta\left(g_{0}\right)$ and $\theta\left(g_{1}\right)$ to $\theta\left(\left(G, \operatorname{pr}_{I}\right)\right)$ and both $\theta\left(\widehat{g_{0}}\right)$ and $\theta\left(\widehat{g_{1}}\right)$ to $\theta\left(\left(\widehat{G}, \operatorname{pr}_{I}\right)\right)$. We have $\Theta\left(l_{0}\right)=\Theta\left(l_{1}\right): \Theta(X, f) \rightarrow$ $\Theta\left(X \times I, f \times \operatorname{id}_{I}\right)$ because of $G$-homotopy invariance of $\Theta$. Now

$$
\begin{aligned}
\Theta & (\widehat{i} \circ i)^{-1} \Theta(\widehat{i})\left(\theta\left(g_{0}\right)\right) \\
& =\Theta\left(l_{0}\right)^{-1} \Theta((\widehat{i} \times \mathrm{id}) \circ(i \times \mathrm{id}))^{-1} \Theta(\widehat{i} \times \mathrm{id}) \Theta\left(l_{0}\right)\left(\theta\left(g_{0}\right)\right) \\
& \left.=\Theta\left(l_{0}\right)^{-1} \Theta(\widehat{i} \times \mathrm{id}) \circ(i \times \mathrm{id})\right)^{-1} \Theta(\widehat{i} \times \mathrm{id})\left(\theta\left(G, \mathrm{pr}_{I}\right)\right) \\
& \left.=\Theta\left(l_{1}\right)^{-1} \Theta(\widehat{i} \times \mathrm{id}) \circ(i \times \mathrm{id})\right)^{-1} \Theta(\widehat{i} \times \mathrm{id}) \Theta\left(l_{1}\right)\left(\theta\left(g_{1}\right)\right) \\
& =\Theta(\hat{i} \circ i)^{-1} \Theta(\widehat{i})\left(\theta\left(g_{1}\right)\right) .
\end{aligned}
$$

Hence $\tau_{Y}$ is well-defined.

Step 2: In this step we define a map

$$
\tau_{d}: U_{G}^{\mathbb{Z}}(X, f) \rightarrow \Theta_{G}(X, f)
$$

for $d \geq 2$. Let $[a: M \rightarrow M \circ \phi] \in U_{G}^{\mathbb{Z}}(X, f)$. Then $M$ is a finite free $\mathbb{Z} \Pi(G, X)$-module, so there is an isomorphism $M \cong \bigoplus_{i \in I} \mathbb{Z} \Pi(G, X)\left(?, x_{i}\right)$ with $\left(x_{i}: G / H_{i} \rightarrow X\right) \in \mathrm{Ob} \Pi(G, X)$ [Lüc89, p. 167]. Set $q_{i}:=x_{i}$ 。 $\operatorname{pr}_{G / H_{i}}: G / H_{i} \times S^{d-1} \rightarrow X$ and define $Y$ to be the pushout

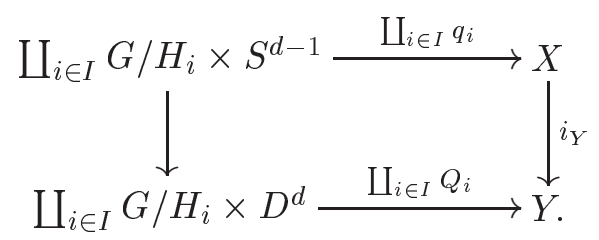

Then there is an isomorphism $c: M \stackrel{\sim}{\rightarrow} \bigoplus_{i \in I} \mathbb{Z} \Pi(G, X)\left(?, x_{i}\right) \stackrel{\sim}{\rightarrow} C_{d}^{c}(\tilde{Y}, \widetilde{X})$. (Remember that $C_{d}^{c}(\widetilde{Y}, \widetilde{X})$ is a free $\mathbb{Z} \Pi(G, X)$-module with equivalence class 
of bases given by the cells of $Y \backslash X$, and the cells of $Y \backslash X$ are given by the $x_{i}$ by construction [Lüc89, Example 9.18].) Then we have $\operatorname{res}_{\phi} c \circ a \circ$ $c^{-1}: C_{d}^{c}(\widetilde{Y}, \widetilde{X}) \rightarrow C_{d}^{c}(\widetilde{Y}, \widetilde{X}) \circ \phi$. Let $[(\widehat{g}, g)]$ be the preimage of $\operatorname{res}_{\phi} c \circ a \circ c^{-1}$ under the isomorphism

$$
\eta:\left[\left(C_{X} Y, Y\right),\left(C_{X} Y, Y\right)\right]_{f}^{G} \stackrel{\sim}{\rightarrow} \operatorname{Mor}_{\mathbb{Z} \Pi(G, X)}\left(C_{d}^{c}(\widetilde{Y}, \widetilde{X}), C_{d}^{c}(\widetilde{Y}, \widetilde{X}) \circ \phi\right) .
$$

Then $\operatorname{res}_{\phi} c \circ a \circ c^{-1}=\eta([(\widehat{g}, g)])=C_{d}^{c}(\widetilde{g}, \widetilde{f})$. So $[a]=\left[\operatorname{res}_{\phi} c \circ a \circ c^{-1}\right]=$ $\left[C_{d}^{c}(\widetilde{g}, \widetilde{f})\right]=\left[(-1)^{d} C^{c}(\widetilde{g}, \widetilde{f})\right]$. Define

$$
\tau_{d}([a]):=(-1)^{d}\left(\tau_{Y}([(\widehat{g}, g)])-\theta(f)\right) .
$$

This definition is independent of the choices made: Let $[a: M \rightarrow M \circ \phi]=$ $\left[a^{\prime}: M^{\prime} \rightarrow M^{\prime} \circ \phi\right]$ with $\tau: a \stackrel{\sim}{\rightarrow} a^{\prime}, M^{\prime} \cong \bigoplus_{i \in I^{\prime}} \mathbb{Z} \Pi(G, X)\left(?, x_{i}^{\prime}\right)$ and $c^{\prime}: M^{\prime} \stackrel{\sim}{\rightarrow} C_{d}^{c}\left(\widetilde{Y^{\prime}}, \widetilde{X}\right)$. Then $c^{\prime} \circ \tau \circ c^{-1}: C_{d}^{c}(\widetilde{Y}, \widetilde{X}) \stackrel{\sim}{\rightarrow} C_{d}^{c}\left(\widetilde{Y^{\prime}}, \widetilde{X}\right)$, and $\eta^{-1}\left(c^{\prime} \circ \tau \circ c^{-1}\right)=[(\widehat{k}, k)] \in\left[\left(C_{X} Y, Y\right),\left(C_{X} Y^{\prime}, Y^{\prime}\right)\right]_{\mathrm{id}_{X}}^{G}$ is represented by a $G$ homotopy equivalence $k: Y \stackrel{\sim}{\rightarrow} Y^{\prime}$ relative $X$. This $G$-homotopy equivalence has a $G$-homotopy inverse given by a representative $l$ of $\eta^{-1}\left(c \circ \tau^{-1} \circ c^{-1}\right)=$ $[(\widehat{l}, l)] \in\left[\left(C_{X} Y^{\prime}, Y^{\prime}\right),\left(C_{X} Y, Y\right)\right]_{\mathrm{id}_{X}}^{G}$. We have the commutative diagram

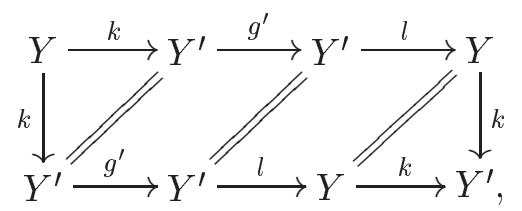

so by invariance under $G$-homotopy equivalence $\Theta(k) \theta\left(Y, l g^{\prime} k\right)=\theta\left(Y^{\prime}, k l g^{\prime}\right)$. Using the compatibility of $\eta$ with compositions established in Remark 4.1.4, we see that

$$
\begin{aligned}
{\left[\left(\widehat{l g^{\prime}} \widehat{k}, l g^{\prime} k\right)\right] } & =[(\widehat{l}, l)] \circ\left[\left(\widehat{g}^{\prime}, g^{\prime}\right)\right] \circ[(\widehat{k}, k)] \\
& =\eta^{-1}\left(\phi^{*} \operatorname{id}^{*}\left(c \tau^{-1} c^{\prime-1}\right) \circ \mathrm{id}^{*}\left(\operatorname{res}_{\phi} c^{\prime} a^{\prime} c^{\prime-1}\right) \circ\left(c^{\prime} \tau c^{-1}\right)\right) \\
& =\eta^{-1}\left(\operatorname{res}_{\phi} c \operatorname{res}_{\phi} \tau^{-1} a^{\prime} \tau c^{-1}\right) \\
& =\eta^{-1}\left(\operatorname{res}_{\phi} c a c^{-1}\right) \\
& =[(\widehat{g}, g)]
\end{aligned}
$$

so $\theta\left(Y, l g^{\prime} k\right)=\theta(Y, g)$ by $G$-homotopy invariance of $\theta$. Also $\left[k l g^{\prime}\right]=\left[g^{\prime}\right]$ since $k$ and $l$ are $G$-homotopy inverse to each other, so $\theta\left(Y^{\prime}, k l g^{\prime}\right)=\theta\left(Y^{\prime}, g^{\prime}\right)$. Thus $\Theta(k) \theta(Y, g)=\theta\left(Y^{\prime}, g^{\prime}\right)$. We want to show that $\tau_{Y}([a])=\tau_{Y^{\prime}}\left(\left[a^{\prime}\right]\right)$, i.e., that

$$
\begin{aligned}
\Theta\left(\widehat{i_{Y}} \circ i_{Y}\right)^{-1} \Theta\left(\widehat{i_{Y}}\right) \theta(g) & =\Theta\left(\widehat{i_{Y^{\prime}}} \circ i_{Y^{\prime}}\right)^{-1} \Theta\left(\widehat{i_{Y^{\prime}}}\right) \theta\left(g^{\prime}\right) \\
& =\Theta\left(\widehat{i_{Y^{\prime}}} \circ i_{Y^{\prime}}\right)^{-1} \Theta\left(\widehat{i_{Y^{\prime}}}\right) \Theta(k) \theta(g) .
\end{aligned}
$$


We obtain a commutative diagram

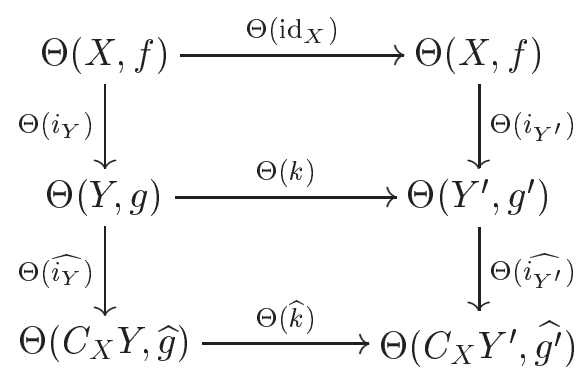

and thus

$$
\begin{aligned}
\Theta\left(\widehat{i_{Y^{\prime}}} \circ i_{Y^{\prime}}\right)^{-1} \Theta\left(\widehat{i_{Y^{\prime}}}\right) \Theta(k) & =\Theta\left(\widehat{i_{Y^{\prime}}} \circ i_{Y^{\prime}}\right)^{-1} \Theta(\widehat{k}) \Theta\left(\widehat{i_{Y}}\right) \\
& =\Theta\left(\operatorname{id}_{X}\right) \Theta\left(\widehat{i_{Y}} \circ i_{Y}\right)^{-1} \Theta\left(\widehat{i_{Y}}\right) \\
& =\Theta\left(\widehat{i_{Y}} \circ i_{Y}\right)^{-1} \Theta\left(\widehat{i_{Y}}\right),
\end{aligned}
$$

so we are finished.

Step 3: To see that $\tau_{d}$ is well-defined, we must see that it is compatible

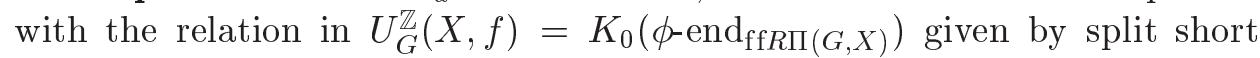
exact sequences. For a split short exact sequence $0 \rightarrow a_{0} \rightarrow a \rightarrow a_{1} \rightarrow 0$ we have $\left[a_{0}\right]+\left[a_{1}\right]=[a]$, and so we need to check that $\tau_{d}\left(\left[a_{0}\right]\right)+\tau_{d}\left(\left[a_{1}\right]\right)=\tau_{d}([a])$.

As above we choose $d$-extensions $Y_{0}$ and $Y_{1}$ and $\mathbb{Z} \Pi(G, X)$-isomorphisms $c_{0}: M_{0} \stackrel{\sim}{\rightarrow} C_{d}^{c}\left(\widetilde{Y_{0}}, \widetilde{X}\right)$ and $c_{1}: M_{1} \stackrel{\sim}{\rightarrow} C_{d}^{c}\left(\widetilde{Y_{1}}, \widetilde{X}\right)$. We put $Y:=Y_{0} \cup_{X} Y_{1}$. We have $C_{X} Y=C_{X} Y_{0} \cup_{X} C_{X} Y_{1}, i=i_{0} \cup_{X} i_{1}$ and $\hat{i}=\hat{i_{0}} \cup_{X} \hat{i_{1}}$, where $i_{0}: X \rightarrow Y_{0}, i_{1}: X \rightarrow Y_{1}, i: X \rightarrow Y, \widehat{i_{0}}: Y_{0} \rightarrow C_{X} Y_{0}, \widehat{i_{1}}: Y_{1} \rightarrow C_{X} Y_{1}$ and $\widehat{i}: Y \rightarrow C_{X} Y$ are the canonical inclusions. Then the direct sum of $c_{0}$ and $c_{1}$ yields a $\mathbb{Z} \Pi(G, X)$-isomorphism $c: M_{0} \oplus M_{1} \stackrel{\sim}{\rightarrow} C_{d}^{c}(\widetilde{Y}, \widetilde{X})$. Let $g: Y \rightarrow Y$ and $g_{0}: Y_{0} \rightarrow Y_{0}$ be maps such that $c_{0}\left(a_{0}\right)=\operatorname{res}_{\phi} c_{0} a_{0} c_{0}^{-1}=C_{d}^{c}\left(\widetilde{g_{0}}, \widetilde{f}\right)$ and $c(a)=\operatorname{res}_{\phi} c a c^{-1}=C_{d}^{c}(\widetilde{g}, \widetilde{f})$. Note that every $\phi$-morphism of $C_{d}^{c}\left(\widetilde{Y_{0}}, \widetilde{X}\right)$ and $C_{d}^{c}(\widetilde{Y}, \widetilde{X})$ can be written as $C_{d}^{c}\left(\widetilde{g_{0}}, \widetilde{f}\right)$ and $C_{d}^{c}(\widetilde{g}, \widetilde{f})$ respectively since $\eta$ is surjective, and that by using the isomorphism $\eta$ we can find an extension $\widehat{g_{0}}$ of $g_{0}$. Denote by $j: Y_{1} \rightarrow C_{X} Y_{0} \cup_{X} Y_{1}$ the canonical inclusion and by $p: C_{X} Y_{0} \cup_{X} Y_{1} \rightarrow Y_{1}$ the canonical projection. Then $c_{1}\left(\left[a_{1}\right]\right)=\left[p \circ\left(\widehat{g_{0}} \cup_{g_{0}}\right.\right.$ $g) \circ j]$. We have the pushout diagram

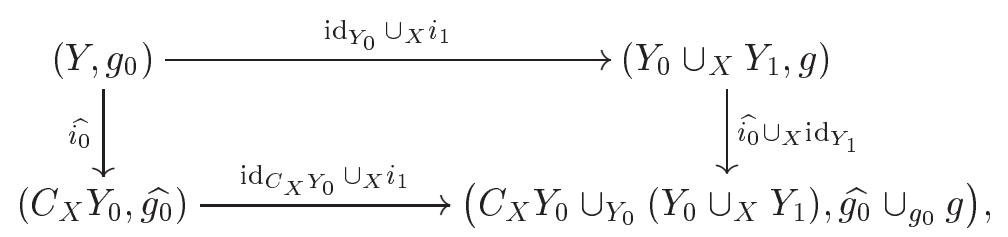

thus by additivity

$$
\begin{aligned}
\theta\left(\widehat{g_{0}} \cup_{g_{0}} g\right)= & \Theta\left(\widehat{i_{0}} \cup_{X} \operatorname{id}_{Y_{1}}\right) \theta(g)+\Theta\left(\operatorname{id}_{C_{X} Y_{0}} \cup_{X} i_{1}\right)\left(\theta\left(\widehat{g_{0}}\right)\right) \\
& -\Theta\left(\left(\operatorname{id}_{C_{X} Y_{0}} \cup_{X} i_{1}\right) \circ \widehat{i_{0}}\right)\left(\theta\left(g_{0}\right)\right) .
\end{aligned}
$$


Applying $\Theta(\widehat{i} \circ i)^{-1} \circ \Theta\left(\operatorname{id}_{C_{X} Y_{0}} \cup_{X} \widehat{i_{1}}\right)$ to this formula and invariance under $G$-homotopy equivalence applied to $\widehat{i_{0}}:(X, f) \rightarrow\left(C_{X} Y_{0}, \widehat{g_{0}}\right)$ yields

$$
\begin{aligned}
& \Theta(\widehat{i} \circ i)^{-1} \circ \Theta\left(\operatorname{id}_{C_{X} Y_{0}} \cup_{X} \widehat{\hat{i_{1}}}\right)\left(\theta\left(\widehat{g_{0}} \cup_{g_{0}} g\right)\right) \\
& \quad=\Theta(\widehat{i} \circ i)^{-1} \circ \Theta(\widehat{i})(\theta(g))+\theta(f)-\Theta\left(\widehat{i_{0}} \circ i_{0}\right)^{-1} \circ \Theta\left(\widehat{i_{0}}\right)\left(\theta\left(g_{0}\right)\right) .
\end{aligned}
$$

The canonical inclusion $j: Y_{1} \rightarrow C_{X} Y_{0} \cup_{X} Y_{1}$ is a $G$-homotopy equivalence, and since $j \circ\left(p \circ\left(\widehat{g_{0}} \cup_{g_{0}} g\right) \circ j\right)=\left(j \circ p \circ\left(\widehat{g_{0}} \cup_{g_{0}} g\right)\right) \circ j$ we obtain $\Theta(j)(\theta(p \circ$ $\left.\left.\left(\widehat{g_{0}} \cup_{g_{0}} g\right) \circ j\right)\right)=\theta\left(j \circ p \circ\left(\widehat{g_{0}} \cup_{g_{0}} g\right)\right)$. The map $j \circ p$ is $G$-homotopic relative $X$ to the identity, so $\theta\left(j \circ p \circ\left(\widehat{g_{0}} \cup_{g_{0}} g\right)\right)=\theta\left(\widehat{g_{0}} \cup_{g_{0}} g\right)$. We see that

$$
\begin{aligned}
& \Theta(\widehat{i} \circ i)^{-1} \circ \Theta\left(\operatorname{id}_{C_{X} Y_{0}} \cup_{X} \widehat{i_{1}}\right)\left(\theta\left(\widehat{g_{0}} \cup_{g_{0}} g\right)\right) \\
& =\Theta(\widehat{i} \circ i)^{-1} \circ \Theta\left(\left(\operatorname{id}_{C_{X} Y_{0}} \cup_{X} \widehat{i_{1}}\right) \circ j\right) \theta\left(p \circ\left(\widehat{g_{0}} \cup_{g_{0}} g\right) \circ j\right) \\
& =\Theta(\widehat{i} \circ i)^{-1} \circ \Theta(\widehat{j}) \Theta(\widehat{i}) \theta\left(p \circ\left(\widehat{g_{0}} \cup_{g_{0}} g\right) \circ j\right) \\
& =\Theta\left(\operatorname{id}_{X}\right) \Theta\left(\widehat{i_{1}} \circ i_{1}\right)^{-1} \circ \Theta\left(\widehat{i_{1}}\right) \theta\left(p \circ\left(\widehat{g_{0}} \cup_{g_{0}} g\right) \circ j\right) .
\end{aligned}
$$

Equation 4.1 now implies

$$
\begin{aligned}
& (-1)^{d}\left(\Theta\left(\widehat{i_{1}} \circ i_{1}\right)^{-1} \circ \Theta\left(\widehat{i_{1}}\right) \theta\left(p \circ\left(\widehat{g_{0}} \cup_{g_{0}} g\right) \circ j\right)-\theta(f)\right) \\
& \quad=(-1)^{d}\left(\left(\Theta(\widehat{i} \circ i)^{-1} \circ \Theta(\widehat{i}) \theta(g)-\theta(f)\right)-\left(\Theta\left(\widehat{i_{0}} \circ i_{0}\right)^{-1} \circ \Theta\left(\widehat{i_{0}}\right) \theta\left(g_{0}\right)-\theta(f)\right)\right),
\end{aligned}
$$

so $\tau_{d}\left(\left[a_{1}\right]\right)=\tau_{d}([a])-\tau_{d}\left(\left[a_{0}\right]\right)$ as was to be shown.

Step 4: Now we need to show that $\tau_{d}$ is independent of the choice of the integer $d \geq 2$. Let $[a] \in U_{G}^{\mathbb{Z}}(X, f)$. It suffices to show that for $d \geq 2$ we have $\tau_{d}([a])=\tau_{d+1}([a])$. Let $(Y, g)$ be a $d$-extension of $X$, chosen as above such that $[a]=\left[C_{d}^{c}(\widetilde{g}, \widetilde{f})\right]$.

We want to describe a suspension map

$$
\begin{aligned}
\Sigma_{X}:\left[\left(C_{X} Y, Y\right),\left(C_{X} Z, Z\right)\right]_{f}^{G} & \rightarrow\left[\left(C_{X} \Sigma_{X} Y, \Sigma_{X} Y\right),\left(C_{X} \Sigma_{X} Z, \Sigma_{X} Z\right)\right]_{f}^{G} \\
{[(\widehat{g}, g)] } & \mapsto\left[\left(\widehat{\Sigma_{X} g}, \Sigma_{X} g\right)\right] .
\end{aligned}
$$

We set $\Sigma_{X} g:=\widehat{g} \cup_{g} \widehat{g}$. The definition of $\widehat{\Sigma_{X}} g$ is a little more complicated. Since $C_{X}$ itself is a pushout construction, it is compatible with pushouts, and thus applying $C_{X}$ to the pushout diagram

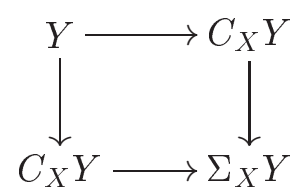

yields

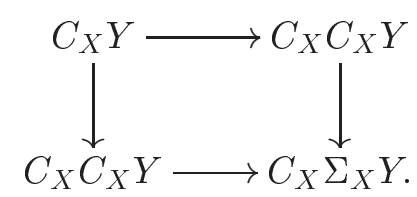


We will define an endomorphism $\bar{g}: C_{X} C_{X} Y \rightarrow C_{X} C_{X} Z$ extending $\widehat{g}$ and then set $\widehat{\Sigma_{X} g}:=\bar{g} \cup_{\widehat{g}} \bar{g}$. For the definition of $\bar{g}$ it is convenient to rewrite $C_{X} C_{X} Y$ as follows. Namely, there is a commutative diagram

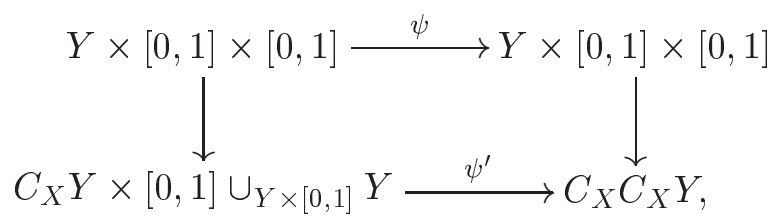

where

$$
\psi(y, s, t)=\left(y, \frac{(1-t) s}{\max \{t, 1-t\}}, \frac{t s}{\max \{t, 1-t\}}\right),
$$

the vertical arrows are the obvious projections and the space in the lower left corner is the pushout of $C_{X} Y \times[0,1] \stackrel{j}{\leftarrow} Y \times[0,1] \stackrel{\mathrm{pr}}{\longrightarrow} Y$ for $j$ and pr being the canonical inclusion and projection. One easily checks that $\psi^{\prime}$ is a homeomorphism. Conjugating the endomorphism $\widehat{g} \times \operatorname{id} \cup_{g \times \text { id }} g$ with $\psi^{\prime}$ yields $\bar{g}$.

If $Y$ is a $d$-extension of $X$, then $\Sigma_{X} Y$ is a $(d+1)$-extension and there is a bijective correspondence between the $d$-cells in $Y \backslash X$ and the $(d+1)$-cells in $\Sigma_{X} Y \backslash X$. In particular, the suspension of $C^{c}(\widetilde{Y}, \widetilde{X})$ is $C^{c}\left(\widetilde{\Sigma_{X} Y}, \widetilde{X}\right)$. The following diagram commutes:

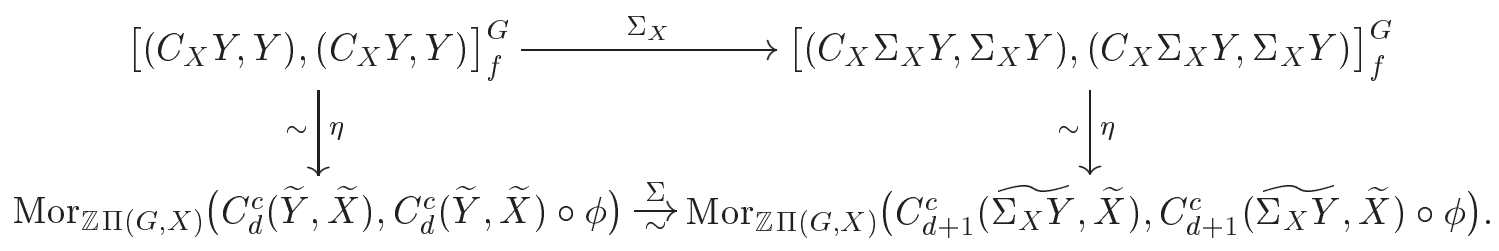

In particular $\Sigma\left(C_{d}^{c}(\widetilde{g}, \widetilde{f})\right)=C_{d+1}^{c}\left(\widetilde{\Sigma_{X} g}, \widetilde{f}\right)$. Since $\Sigma$ is an isomorphism, $\left[C_{d}^{c}(\widetilde{g}, \widetilde{f})\right]=\left[\Sigma\left(C_{d}^{c}(\widetilde{g}, \widetilde{f})\right)\right]$. We see that

$$
[a]=\left[C_{d}^{c}(\widetilde{g}, \widetilde{f})\right]=\left[\Sigma\left(C_{d}^{c}(\widetilde{g}, \widetilde{f})\right)\right]=\left[C_{d+1}^{c}\left(\widetilde{\Sigma_{X} g}, \widetilde{f}\right)\right],
$$

so

$$
\begin{aligned}
\tau_{d}([a]) & =(-1)^{d}\left(\tau_{Y}([(\widehat{g}, g)])-\theta(f)\right) \\
\text { and } \quad \tau_{d+1}([a]) & =(-1)^{d+1}\left(\tau_{\Sigma_{X} Y}\left(\left[\left(\widehat{\Sigma_{X} g}, \Sigma_{X} g\right)\right]\right)-\theta(f)\right) .
\end{aligned}
$$

Let us look at the pushout diagram

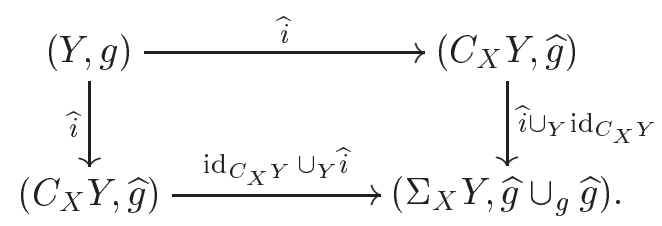


Remember that $\Sigma_{X} g=\widehat{g} \cup_{g} \widehat{g}$. Additivity of $\Theta$ yields

$$
\left.\theta\left(\Sigma_{X} g\right)=\Theta\left(\operatorname{id}_{C_{X} Y} \cup_{Y} \widehat{i}\right)(\theta(\widehat{g}))-\Theta \widehat{i} \cup_{Y} \operatorname{id}_{C_{X} Y}\right)(\theta(\widehat{g}))-\Theta\left(\widehat{i} \cup_{Y} \widehat{i}\right)(\theta(g)) .
$$

We obtain

$$
\begin{aligned}
& \Theta\left(\widehat{i_{\Sigma_{X} Y}} \circ i_{\Sigma_{X} Y}\right)^{-1} \Theta\left(\widehat{i_{\Sigma_{X} Y}}\right)\left(\theta\left(\Sigma_{X} g\right)\right) \\
&=\quad \Theta\left(\widehat{i_{\Sigma_{X} Y}} \circ i_{\Sigma_{X} Y}\right)^{-1} \Theta\left(\widehat{i_{\Sigma_{X} Y}} \circ\left(\operatorname{id}_{C_{X} Y} \cup_{Y} \widehat{i}\right)\right)(\theta(\widehat{g})) \\
&+\Theta\left(\widehat{i_{\Sigma_{X} Y}} \circ i_{\Sigma_{X} Y}\right)^{-1} \Theta\left(\widehat{i_{\Sigma_{X} Y}} \circ\left(\widehat{i} \cup_{Y} \operatorname{id}_{C_{X} Y}\right)\right)(\theta(\widehat{g})) \\
&\left.\quad-\Theta\left(\widehat{i_{\Sigma_{X} Y}} \circ i_{\Sigma_{X} Y}\right)^{-1} \Theta\left(\widehat{i_{\Sigma_{X} Y}} \circ \widehat{(i} \cup_{Y} \widehat{i}\right)\right)(\theta(g)) \\
&= 2 \theta(f)-\theta\left(\widehat{i_{Y}} \circ i_{Y}\right)^{-1} \Theta\left(\widehat{i_{Y}}\right)(\theta(g))
\end{aligned}
$$

by the same procedure as before (using appropriate commutative diagrams and $G$-homotopy invariance of $\Theta$ ), so

$$
\tau_{\Sigma_{X} Y}\left(\left[\left(\widehat{\Sigma_{X}} g, \Sigma_{X} g\right)\right]\right)-\theta(f)=-\left(\tau_{Y}([(\widehat{g}, g)])-\theta(f)\right)
$$

which implies $\tau_{d}([a])=\tau_{d+1}([a])$. So $\tau_{d}$ is independent of $d \geq 2$, and we can define $\tau:=\tau_{d}$ for $d \geq 2$.

Step 5: We have defined a map $\tau_{G(X, f)}: U_{G}^{\mathbb{Z}}(X, f) \rightarrow \Theta_{G}(X, f)$ for $(X, f)$ in $\operatorname{End}\left(G-\mathcal{C} W_{\mathrm{fp}}\right)$, for discrete groups $G$. We want to show that $\tau$ is actually a natural transformation from $U_{G}^{\mathbb{Z}}$ to $\Theta_{G}$, so we must check that for a morphism in our category, i.e., a $G$-equivariant map $h$ such that

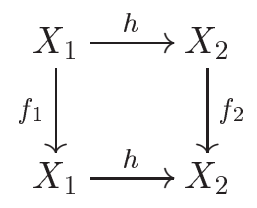

commutes, the diagram

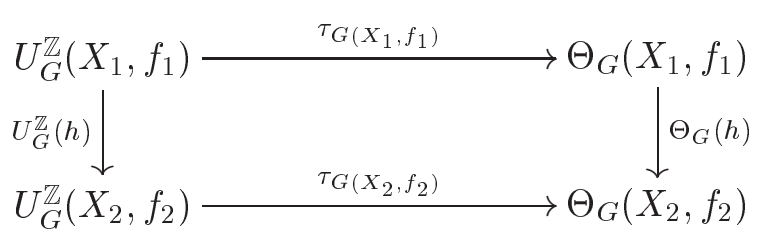

commutes. Let $[a] \in U_{G}^{\mathbb{Z}}\left(X_{1}, f_{1}\right)$, and let $Y_{1}$ be a $d$-extension $(d \geq 2)$ of $X_{1}$ with endomorphism $g_{1}$ extending $f_{1}$, i.e., $\left.g_{1}\right|_{X_{1}}=f_{1}$, such that $\left[C_{d}^{c}\left(\widetilde{g_{1}}, \widetilde{f_{1}}\right)\right]=$ $[a]$. Define $\left(Y_{2}, g_{2}\right)$ as the pushout

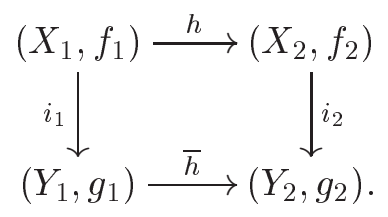


We would like to use $C_{d}^{c}\left(\widetilde{g_{2}}, \widetilde{f}_{2}\right)$ as a representative of $U_{G}^{\mathbb{Z}}(h)([a])$. By additivity of $(U, u)$ applied to this diagram, we have

$$
\begin{aligned}
u_{G}^{\mathbb{Z}}\left(g_{2}\right) & =U_{G}^{\mathbb{Z}}(\bar{h}) u_{G}^{\mathbb{Z}}\left(g_{1}\right)+U_{G}^{\mathbb{Z}}\left(i_{2}\right) u_{G}^{\mathbb{Z}}\left(f_{2}\right)-U_{G}^{\mathbb{Z}}\left(i_{2} h\right) u_{G}^{\mathbb{Z}}\left(f_{1}\right), \text { i.e. }, \\
{\left[C^{c}\left(\widetilde{g_{2}}\right)\right] } & =\left[\bar{H}_{*} C^{c}\left(\widetilde{g_{1}}\right)\right]+\left[I_{2 *} C^{c}\left(\widetilde{f}_{2}\right)\right]-\left[I_{2 *} H_{*} C^{c}\left(\widetilde{f}_{1}\right)\right] \in U_{G}^{\mathbb{Z}}\left(Y_{2}, g_{2}\right) .
\end{aligned}
$$

This implies

$$
\left[\operatorname{res}_{I_{2}} C^{c}\left(\widetilde{g_{2}}\right)\right]=\left[\operatorname{res}_{I_{2}} \bar{H}_{*} C^{c}\left(\widetilde{g_{1}}\right)\right]+\left[\operatorname{res}_{I_{2}} I_{2 *} C^{c}\left(\widetilde{f}_{2}\right)\right]-\left[\operatorname{res}_{I_{2}} I_{2 *} H_{*} C^{c}\left(\widetilde{f}_{1}\right)\right] .
$$

We now use the fact that $I_{1}=\Pi\left(G, i_{1}\right): \Pi\left(G, X_{1}\right) \rightarrow \Pi\left(G, Y_{1}\right)$ and $I_{2}=$ $\Pi\left(G, i_{2}\right): \Pi\left(G, X_{2}\right) \rightarrow \Pi\left(G, Y_{2}\right)$ are equivalences of categories. By Proposition 2.2.4, this implies that

$$
\text { and } \begin{aligned}
I_{1 *}: \phi_{1}-\operatorname{end}_{\mathrm{ff} R \Pi\left(G, X_{1}\right)} \rightarrow \psi_{1}-\operatorname{end}_{\mathrm{ff} R \Pi\left(G, Y_{1}\right)} \\
I_{2 *}: \phi_{2}-\operatorname{end}_{\mathrm{ff} R \Pi\left(G, X_{2}\right)} \rightarrow \psi_{2}-\operatorname{end}_{\mathrm{ff} R \Pi\left(G, Y_{2}\right)}
\end{aligned}
$$

are also equivalences of categories. It can be read off the proof of Proposition 2.2.4 that $I_{1 *} \operatorname{res}_{I_{1}} l \cong l$ for all $l \in \psi_{1}-\operatorname{end}_{\mathrm{ff} R \Pi\left(G, Y_{1}\right)}$ and that $I_{2 *} \operatorname{res}_{I_{2}} I_{2 *} k \cong$ $I_{2 *} k$ for all $k \in \phi_{2}$-end $\operatorname{ff}_{\mathrm{ff} \Pi\left(G, X_{2}\right)}$ which implies that $\left[\operatorname{res}_{I_{2}} I_{2 *} k\right]=[k]$ by injectivity of $I_{2 *}$ on isomorphism classes. So $\left[\operatorname{res}_{I_{2}} I_{2 *} C^{c}\left(\widetilde{f}_{2}\right)\right]=\left[C^{c}\left(\widetilde{f}_{2}\right)\right]$ and $\left[\operatorname{res}_{I_{2}} I_{2 *} H_{*} C^{c}\left(\widetilde{f}_{1}\right)\right]=\left[H_{*} C^{c}\left(\widetilde{f}_{1}\right)\right]$. Using Lemma 2.2 .2 we see that $\bar{H}_{*} I_{1 *}=\left(\bar{H} I_{1}\right)_{*}=\left(I_{2} H\right)_{*}=I_{2 *} H_{*}$ and we obtain

$$
\begin{aligned}
{\left[\operatorname{res}_{I_{2}} \bar{H}_{*} C^{c}\left(\widetilde{g_{1}}\right)\right] } & =\left[\operatorname{res}_{I_{2}} \bar{H}_{*} I_{1 *} \operatorname{res}_{I_{1}} C^{c}\left(\widetilde{g_{1}}\right)\right]=\left[\operatorname{res}_{I_{2}} I_{2 *} H_{*} \operatorname{res}_{I_{1}} C^{c}\left(\widetilde{g_{1}}\right)\right] \\
& =\left[H_{*} \operatorname{res}_{I_{1}} C^{c}\left(\widetilde{g_{1}}\right)\right] .
\end{aligned}
$$

We conclude that

$$
\left[\operatorname{res}_{I_{2}} C^{c}\left(\widetilde{g_{2}}\right)\right]=\left[H_{*} \operatorname{res}_{I_{1}} C^{c}\left(\widetilde{g_{1}}\right)\right]+\left[C^{c}\left(\widetilde{f_{2}}\right)\right]-\left[H_{*} C^{c}\left(\widetilde{f_{1}}\right)\right] .
$$

Using additivity applied to the short exact sequences defining the relative maps and the fact that $C^{c}(\widetilde{g}, \widetilde{f})$ is concentrated in degree $d$, we see that

$$
\begin{aligned}
U_{G}^{\mathbb{Z}}(h)\left(\left[C_{d}^{c}\left(\widetilde{g_{1}}, \widetilde{f_{1}}\right)\right]\right) & =U_{G}^{\mathbb{Z}}(h)\left(\left[(-1)^{d} C^{c}\left(\widetilde{g_{1}}, \widetilde{f_{1}}\right)\right]\right) \\
& =(-1)^{d}\left(\left[H_{*} \operatorname{res}_{I_{1}} C^{c}\left(\widetilde{g_{1}}\right)\right]-\left[H_{*} C^{c}\left(\widetilde{f_{1}}\right)\right]\right) \\
& =(-1)^{d}\left(\left[\operatorname{res}_{I_{2}} C^{c}\left(\widetilde{g_{2}}\right)\right]-\left[C^{c}\left(\widetilde{f_{2}}\right)\right]\right) \\
& =(-1)^{d}\left(\left[C^{c}\left(\widetilde{g_{2}}, \widetilde{f_{2}}\right)\right]\right) \\
& =\left[C_{d}^{c}\left(\widetilde{g_{2}}, \widetilde{f_{2}}\right)\right] .
\end{aligned}
$$

Since this is the case,

$$
\begin{aligned}
& \left(\tau_{G\left(X_{2}, f_{2}\right)} \circ U_{G}^{\mathbb{Z}}(h)\right)([a]) \\
& \quad=\left(\tau_{G\left(X_{2}, f_{2}\right)} \circ U_{G}^{\mathbb{Z}}(h)\right)\left(\left[C_{d}^{c}\left(\widetilde{g_{1}}, \widetilde{f_{1}}\right)\right]\right) \\
& \quad=\tau_{G\left(X_{2}, f_{2}\right)}\left(\left[C_{d}^{c}\left(\widetilde{g_{2}}, \widetilde{f_{2}}\right)\right]\right) \\
& \quad=(-1)^{d}\left(\Theta_{G}\left(\widehat{i_{2}} \circ i_{2}\right)^{-1} \Theta_{G}\left(\widehat{i_{2}}\right)\left(\theta_{G}\left(g_{2}\right)\right)-\theta_{G}\left(f_{2}\right)\right),
\end{aligned}
$$


whereas

$$
\begin{aligned}
& \left(\Theta_{G}(h) \circ \tau_{G\left(X_{1}, f_{1}\right)}\right)([a]) \\
& \quad=(-1)^{d}\left(\Theta_{G}(h) \Theta_{G}\left(\widehat{i_{1}} \circ i_{1}\right)^{-1} \Theta_{G}\left(\widehat{i_{1}}\right)\left(\theta_{G}\left(g_{1}\right)\right)-\Theta_{G}(h)\left(\theta_{G}\left(f_{1}\right)\right)\right) .
\end{aligned}
$$

Additivity applied to the commutative diagram

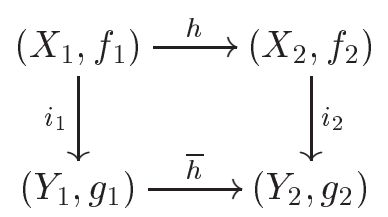

yields $\theta_{G}\left(g_{2}\right)=\Theta_{G}(\bar{h}) \theta_{G}\left(g_{1}\right)+\Theta_{G}\left(i_{2}\right) \theta_{G}\left(f_{2}\right)-\Theta_{G}\left(i_{2} h\right) \theta_{G}\left(f_{1}\right)$, and we apply $\Theta_{G}\left(\widehat{i_{2}} \circ i_{2}\right)^{-1} \Theta_{G}\left(\hat{i_{2}}\right)$ to get

$$
\begin{aligned}
& \Theta_{G}\left(\widehat{i_{2}} \circ i_{2}\right)^{-1} \Theta_{G}\left(\widehat{i_{2}}\right)\left(\theta_{G}\left(g_{2}\right)\right)-\theta_{G}\left(f_{2}\right) \\
& \quad=\Theta_{G}\left(\widehat{i_{2}} \circ i_{2}\right)^{-1} \Theta_{G}\left(\hat{i_{2}} \bar{h}\right)\left(\theta_{G}\left(g_{1}\right)\right)-\Theta_{G}(h)\left(\theta_{G}\left(f_{1}\right)\right) \\
& \quad=\Theta_{G}(h) \Theta_{G}\left(\widehat{i_{1}} \circ i_{1}\right)^{-1} \Theta_{G}\left(\widehat{i_{1}}\right)\left(\theta_{G}\left(g_{1}\right)\right)-\Theta_{G}(h)\left(\theta_{G}\left(f_{1}\right)\right),
\end{aligned}
$$

so indeed $\left(\tau_{G\left(X_{2}, f_{2}\right)} \circ U_{G}^{\mathbb{Z}}(h)\right)([a])=\left(\Theta_{G}(h) \circ \tau_{G\left(X_{1}, f_{1}\right)}\right)([a])$.

Step 6: Next we need to show that the natural transformation $\tau: U_{G}^{\mathbb{Z}} \rightarrow$ $\Theta_{G} \operatorname{maps} u_{G}^{\mathbb{Z}}(X, f) \in U_{G}^{\mathbb{Z}}(X, f)$ to $\theta_{G}(X, f) \in \Theta_{G}(X, f)$ for all $(X, f)$ in $\operatorname{End}\left(G-\mathcal{C} W_{\mathrm{fp}}\right)$, for discrete groups $G$. For a finite $G$-CW-complex $X$ with endomorphism $f: X \rightarrow X$ let $Y_{n}$ be the pushout

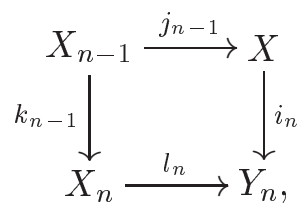

where all arrows are canonical inclusions. There is a canonical retraction $r_{n}: Y_{n} \rightarrow X$ induced by the inclusions of $X_{n-1}, X_{n}$ and $X$ into $X$. Defining $f_{k}: X_{k} \rightarrow X_{k}$ as the restriction of $f$ to $X_{k}$, one obtains an endomorphism $g_{n}: Y_{n} \rightarrow Y_{n}$ as $g_{n}=f_{n} \cup_{f_{n-1}} f$. Additivity applied to the pushout diagram yields

$$
\theta\left(g_{n}\right)=\Theta\left(l_{n}\right) \theta\left(f_{n}\right)+\Theta\left(i_{n}\right) \theta(f)-\Theta\left(l_{n} \circ k_{n-1}\right) \theta\left(f_{n}\right)
$$

and thus

$$
\Theta\left(r_{n}\right) \theta\left(g_{n}\right)=\Theta\left(i_{n}\right) \theta\left(f_{n}\right)+\theta(f)-\Theta\left(i_{n-1}\right) \theta\left(f_{n-1}\right) .
$$

Summing up, we obtain

$$
\sum_{n=0}^{\operatorname{dim}(X)}\left(\Theta\left(r_{n}\right) \theta\left(g_{n}\right)-\theta(f)\right)=\theta(f) \in \Theta(X, f) .
$$


Analogously

$$
\sum_{n=0}^{\operatorname{dim}(X)}\left(U^{\mathbb{Z}}\left(r_{n}\right) u^{\mathbb{Z}}\left(g_{n}\right)-u^{\mathbb{Z}}(f)\right)=u^{\mathbb{Z}}(f) \in U^{\mathbb{Z}}(X, f) .
$$

So we are finished if we can show that

$$
\tau_{G(X, f)}\left(U^{\mathbb{Z}}\left(r_{n}\right) u^{\mathbb{Z}}\left(g_{n}\right)-u^{\mathbb{Z}}(f)\right)=\Theta\left(r_{n}\right) \theta\left(g_{n}\right)-\theta(f) \text { for } 0 \leq n \leq \operatorname{dim}(X) .
$$

If $n \geq 2$ (and thus $I_{n}:=\Pi\left(G, i_{n}\right)$ is an equivalence of categories), then

$$
\begin{aligned}
{\left[C^{c}\left(\widetilde{g_{n}}, \widetilde{f}\right)\right] } & =\left[\operatorname{res}_{I_{n}} C^{c}\left(\widetilde{g_{n}}\right)\right]-\left[C^{c}(\widetilde{f})\right] \\
& =\left[\operatorname{id}_{*} \operatorname{res}_{I_{n}} C^{c}\left(\widetilde{g_{n}}\right)\right]-\left[C^{c}(\widetilde{f})\right] \\
& =\left[R_{n *} I_{n *} \operatorname{res}_{I_{n}} C^{c}\left(\widetilde{g_{n}}\right)\right]-\left[C^{c}(\widetilde{f})\right] \\
& =\left[R_{n *} C^{c}\left(\widetilde{g_{n}}\right)\right]-\left[C^{c}(\widetilde{f})\right] \\
& =U^{\mathbb{Z}}\left(r_{n}\right) u^{\mathbb{Z}}\left(g_{n}\right)-u^{\mathbb{Z}}(f) .
\end{aligned}
$$

This implies that

$$
\begin{aligned}
& \tau_{G(X, f)}\left(U^{\mathbb{Z}}\left(r_{n}\right) u^{\mathbb{Z}}\left(g_{n}\right)-u^{\mathbb{Z}}(f)\right) \\
& \quad=\tau_{G(X, f)}\left(\left[C^{c}\left(\widetilde{g_{n}}, \widetilde{f}\right)\right]\right) \\
& \quad=\tau_{G(X, f)}\left(\left[(-1)^{d} C_{d}^{c}\left(\widetilde{g_{n}}, \widetilde{f}\right)\right]\right) \\
& \quad=(-1)^{d}(-1)^{d}\left(\Theta\left(\widehat{i_{n}} i_{n}\right)^{-1} \circ \Theta\left(\widehat{i_{n}}\right) \theta\left(g_{n}\right)-\theta(f)\right) \\
& \quad=\Theta\left(r_{n}\right) \theta\left(g_{n}\right)-\theta(f) .
\end{aligned}
$$

The last equation follows because we have strict commutativity $r_{n} g_{n}=f r_{n}$, so we can define an endomorphism $\widehat{g_{n}}: C_{X} Y_{n} \rightarrow C_{X} Y_{n}$ extending $g_{n}$ as $g_{n} \times \operatorname{id}_{[0,1]} \cup_{g_{n}} f$, and for $\widehat{r_{n}}: C_{X} Y_{n} \rightarrow X$ we also have $\widehat{r_{n}} \widehat{g_{n}}=f \widehat{r_{n}}$. Thus we can use the commutative diagram of spaces with endomorphisms

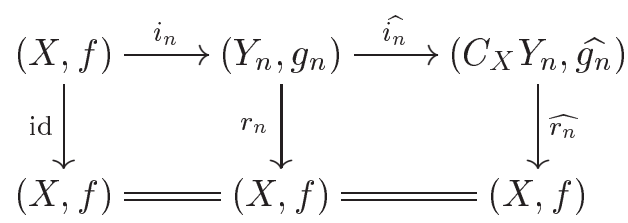

to establish

$$
\Theta\left(\widehat{i_{n}} i_{n}\right)^{-1} \Theta\left(\widehat{i_{n}}\right)=\Theta(\mathrm{id}) \Theta\left(\widehat{i_{n}} i_{n}\right)^{-1} \Theta\left(\widehat{i_{n}}\right)=\Theta\left(\widehat{r_{n}}\right) \Theta\left(\widehat{i_{n}}\right)=\Theta\left(r_{n}\right) .
$$

If $n \in\{0,1\}$, we can use the suspension $\Sigma_{X} Y_{n}$ of $Y_{n}$ to obtain the desired result. As already mentioned before, $\Sigma_{X} Y_{n}$ is defined to be the pushout

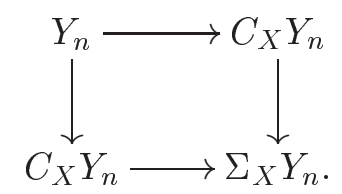


We have an endomorphism $\widehat{g_{n}}: C_{X} Y_{n} \rightarrow C_{X} Y_{n}$, so we also have an endomorphism $\Sigma_{X} g_{n}:=\widehat{g_{n}} \cup_{g_{n}} \widehat{g_{n}}$ of $\Sigma_{X} Y_{n}$. Additivity, application of $\Sigma_{X} r_{n}:=$ $\widehat{r_{n}} \cup_{r_{n}} \widehat{r_{n}}$ and invariance under $G$-homotopy equivalence yield

$$
\begin{aligned}
\Theta\left(\Sigma_{X} r_{n}\right) \theta\left(\Sigma_{X} g_{n}\right) & =2 \theta(f)-\Theta\left(r_{n}\right) \theta\left(g_{n}\right) \\
\text { and } \quad U^{\mathbb{Z}}\left(\Sigma_{X} r_{n}\right) u^{\mathbb{Z}}\left(\Sigma_{X} g_{n}\right) & =2 u^{\mathbb{Z}}(f)-U^{\mathbb{Z}}\left(r_{n}\right) u^{\mathbb{Z}}\left(g_{n}\right) .
\end{aligned}
$$

The retractive space $\Sigma_{X} Y$ is an $(n+1)$-extension of $X$, so applying $\Sigma_{X}$ once or twice gets us into the range $d \geq 2$ where we can use the above results. So we have shown

$$
\tau_{G(X, f)}\left(u_{G}^{\mathbb{Z}}(X, f)\right)=\theta_{G}(X, f) .
$$

Step 7: We have to show that $\tau_{K} \circ U(\alpha)=\Theta(\alpha) \circ \tau_{G}$ for any inclusion $\alpha: G \rightarrow K$. By definition of the natural transformation $\tau$ and of $U(\alpha)$ and using the notation established above, this is equivalent to

$$
\begin{aligned}
& (-1)^{d}\left(\Theta_{K}\left(\widehat{\operatorname{ind}_{\alpha} i} \circ \operatorname{ind}_{\alpha} i\right)^{-1} \Theta_{K}\left(\widehat{\operatorname{ind}_{\alpha} i}\right)\left(\theta_{K}\left(\operatorname{ind}_{\alpha} g\right)\right)-\theta_{K}\left(\operatorname{ind}_{\alpha} f\right)\right) \\
& =\Theta(\alpha)\left((-1)^{d}\left(\Theta_{G}(\widehat{i} \circ i)^{-1} \Theta_{G}(\widehat{i})\left(\theta_{G}(g)\right)-\theta_{G}(f)\right)\right) .
\end{aligned}
$$

We use the fact that the retractive space constructions commute with induction. For any $G$-equivariant map $h: X \rightarrow Y$ of $G$-CW-complexes we have a commutative diagram

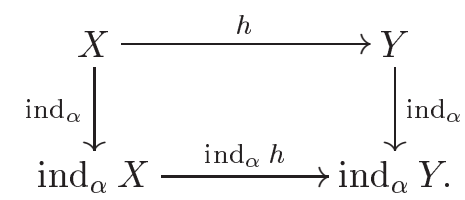

Using the fact that $\Theta$ is a family of functors on $\operatorname{End}\left(G-\mathcal{C} W_{\mathrm{fp}}\right)$ for discrete groups $G$ which is compatible with the induction structure, we obtain the equation $\Theta(\alpha) \Theta_{G}(h)=\Theta_{K}\left(\operatorname{ind}_{\alpha} h\right) \Theta(\alpha)$. So we have

$$
\begin{aligned}
& \Theta(\alpha)\left((-1)^{d}\left(\Theta_{G}(\widehat{i} \circ i)^{-1} \Theta_{G} \widehat{(i)}\left(\theta_{G}(g)\right)-\theta_{G}(f)\right)\right) \\
& =(-1)^{d}\left(\Theta_{K}\left(\widehat{\operatorname{ind}_{\alpha} i} \circ \operatorname{ind}_{\alpha} i\right)^{-1} \Theta_{K}\left(\widehat{\operatorname{ind}_{\alpha} i}\right) \Theta(\alpha)\left(\theta_{G}(g)\right)-\Theta(\alpha) \theta_{G}(f)\right) .
\end{aligned}
$$

Since $(\Theta, \theta)$ is a functorial equivariant Lefschetz invariant, we have the equation $\Theta(\alpha)\left(\theta_{G}(g)\right)=\theta_{K}\left(\operatorname{ind}_{\alpha} g\right)$ and the analogous equation for $f$, so we are finished.

Step 8: The natural transformation $\tau$ is uniquely determined by equation 4.2. As we already saw, any element $[a]$ in $U_{G}^{\mathbb{Z}}(X, f)$ can be realized by 
a $d$-extension $Y$ for $d \geq 2$ as

$$
\begin{aligned}
{[a] } & =\left[C_{d}^{c}(\widetilde{g}, \widetilde{f})\right] \\
& =(-1)^{d}\left[C^{c}(\widetilde{g}, \widetilde{f})\right] \\
& =(-1)^{d}\left(\left[\operatorname{res}_{I} C^{c}(\widetilde{g})\right]-\left[C^{c}(\widetilde{f})\right]\right) \\
& =(-1)^{d}\left(\left[\operatorname{res}_{I} \operatorname{res}_{\widehat{I}} \widehat{I}_{*} C^{c}(\widetilde{g})\right]-\left[C^{c}(\widetilde{f})\right]\right) \\
& \left.=(-1)^{d}\left(\left[\left((\widehat{I I})_{*}\right)^{-1}(\widehat{I} I)_{*} \operatorname{res}_{\widehat{I I}} \widehat{I}_{*} C^{c}(\widetilde{g})\right]-C^{c}(\widetilde{f})\right]\right) \\
& =(-1)^{d}\left(\left[\left((\widehat{I I})_{*}\right)^{-1} \widehat{I}_{*} C^{c}(\widetilde{g})\right]-\left[C^{c}(\widetilde{f})\right]\right) \\
& =(-1)^{d}\left(U_{G}^{\mathbb{Z}}(\widehat{i} \circ i)^{-1} \circ U_{G}^{\mathbb{Z}}(\widehat{i})\left(u_{G}^{\mathbb{Z}}(g)\right)-u_{G}^{\mathbb{Z}}(f)\right),
\end{aligned}
$$

and then by equation 4.2 and functoriality it follows that

$$
\tau_{G(X, f)}([a])=(-1)^{d}\left(\Theta_{G}(\widehat{i} \circ i)^{-1} \circ \Theta_{G} \widehat{(i)}\left(\theta_{G}(g)\right)-\theta_{G}(f)\right) .
$$




\section{Chapter 5}

\section{Splitting Results}

The goal of this chapter is to obtain a direct sum decomposition of the abelian group $K_{0}\left(\phi\right.$-end $\left._{\mathrm{ff} R \Gamma}\right)$ making it more accessible to computations. We obtain a splitting

$$
\begin{aligned}
K_{0}\left(\phi-\operatorname{end}_{\mathrm{ffR}}\right) & \cong \bigoplus_{W \in I_{\Gamma}} K_{0}\left(\phi_{W}-\operatorname{end}_{\mathrm{ff} R W}\right) \\
& \cong \bigoplus_{W \in I_{\Gamma}}\left(K_{0}\left(\phi_{T_{W}}-\operatorname{end}_{\mathrm{ffR} T_{W}}\right) \oplus K_{0}\left(\text { ff } R Q_{W}\right)\right) .
\end{aligned}
$$

The indexing set $I_{\Gamma}$ is a set of equivalence classes of Is $\Gamma$. As usual, Is $\Gamma$ denotes the set of isomorphism classes of $\Gamma$. The equivalence relation introduced on Is $\Gamma$ is described in Section 5.1, as well as the meaning of $W$, $T_{W}$ and $Q_{W}$. Splitting functors which induce the above isomorphism are defined in Section 5.2. The highlight of this chapter are the splitting theorems, Theorem 5.3.1 and Theorem 5.4.2. Finally, we treat a small example in Section 5.5.

\subsection{An Equivalence Relation}

We would like to split $K_{0}\left(\phi-\operatorname{end}_{\mathrm{ff} R \Gamma}\right)$ up into a direct sum indexed by Is $\Gamma$ as in [Lüc89, Theorem 10.34]. But the endofunctor $\phi: \Gamma \rightarrow \Gamma$ does not always respect this direct sum decomposition. It induces a map $\bar{\phi}$ : Is $\Gamma \rightarrow$ Is $\Gamma$ on the isomorphism classes of $\Gamma$, and we look at the orbits of the isomorphism classes $\bar{x} \in$ Is $\Gamma$ under $\bar{\phi}$. Putting all $\bar{x}$ that eventually have the same orbit into an equivalence class, we obtain a partition $I_{\Gamma}=\left\{W_{i}\right\}_{W_{i} \in I_{\Gamma}}$ of Is $\Gamma$.

Definition 5.1.1. Let $\sim_{\phi}$ be the equivalence relation on Is $\Gamma$ defined by

$$
\bar{x} \sim_{\phi} \bar{y}: \Leftrightarrow \text { there exist } n, m \in \mathbb{N} \text { such that } \overline{\phi^{n}(x)}=\overline{\phi^{m}(y)} .
$$

Then we define the set $I_{\Gamma}$ to be $I_{\Gamma}:=$ Is $\Gamma / \sim_{\phi}$. Equivalence classes contained in this set $I_{\Gamma}$ are called $W_{i} \in I_{\Gamma}$. 
There is a partial ordering on $\Gamma$ given by $x \leq y \Leftrightarrow \operatorname{Mor}_{\Gamma}(x, y) \neq \emptyset$. This induces a partial ordering on Is $\Gamma$ given by $\bar{x} \leq \bar{y} \Leftrightarrow \operatorname{Mor}_{\Gamma}(x, y) \neq \emptyset$. We call the endofunctor $\phi$ order-respecting if it respects this partial ordering in the following sense:

Definition 5.1.2. The endofunctor $\phi: \Gamma \rightarrow \Gamma$ is called order-respecting if

1. $\overline{\phi^{m}(x)} \leq \bar{x}$ or $\overline{\phi^{m}(x)} \geq \bar{x} \Longrightarrow \overline{\phi^{m}(x)}=\bar{x}$, for all $m \in \mathbb{N}$.

2. $\bar{x}<\bar{y} \Longrightarrow \overline{\phi(x)}<\overline{\phi(y)}$.

The endofunctors $\phi$ defined by $\phi:=\Pi(G, f)$ arising from the geometric applications as in Chapter 3 are order-respecting since they preserve orbit types.

If $\phi$ is order-respecting, the partial ordering on Is $\Gamma$ induces a partial ordering on the set of equivalence classes $I_{\Gamma}=\left\{W_{i}\right\}_{W_{i} \in I_{\Gamma}}$.

Definition 5.1.3. We write $W_{j} \leq W_{i}$ if and only if there exist $\bar{y} \in W_{j}$ and $\bar{x} \in W_{i}$ such that $\bar{y} \leq \bar{x}$.

This relation " $\leq$ " is well-defined, which we prove in the following lemma.

Lemma 5.1.4. The relation " $\leq$ " of Definition 5.1 .3 is well-defined.

Proof. For $i \neq j$, let $\bar{x} \in W_{i}$ and $\bar{y} \in W_{j}$ with $\bar{y} \leq \bar{x}$. Assume that there are $\overline{x^{\prime}} \in W_{i}$ and $\overline{y^{\prime}} \in W_{j}$ with $\overline{x^{\prime}} \leq \overline{y^{\prime}}$. Since $\bar{x}$ and $\overline{x^{\prime}}$ are in $W_{i}$, we have $n, n^{\prime} \in \mathbb{N}$ such that $\overline{\phi^{n}(x)}=\overline{\phi^{n^{\prime}}\left(x^{\prime}\right)}$, so $\overline{\phi^{n}(y)} \leq \overline{\phi^{n}(x)}=\overline{\phi^{n^{\prime}}\left(x^{\prime}\right)} \leq \overline{\phi^{n^{\prime}}\left(y^{\prime}\right)}$.

Since $\overline{\phi^{n}(y)}$ and $\overline{\phi^{n^{\prime}}\left(y^{\prime}\right)}$ are in $W_{j}$, there are $m, m^{\prime} \in \mathbb{N}$ such that $\overline{\phi^{n+m}(y)}=\overline{\phi^{n^{\prime}+m^{\prime}}\left(y^{\prime}\right)}$. This implies that we have the inequality $\overline{\phi^{n+m^{\prime}}(y)} \leq$ $\overline{\phi^{n+m^{\prime}}(x)}=\overline{\phi^{n^{\prime}+m^{\prime}}\left(x^{\prime}\right)} \leq \overline{\phi^{n^{\prime}+m^{\prime}}\left(y^{\prime}\right)}=\overline{\phi^{n+m}(y)}$.

Since $\phi$ is order-respecting, this has to be an equality, thus $\overline{\phi^{n+m^{\prime}}(y)}=$ $\overline{\phi^{n+m^{\prime}}(x)} \in W_{i}$ and $\overline{\phi^{n+m^{\prime}}(y)} \in W_{j}$, a contradiction to $i \neq j$.

We index the $W_{i}$ such that $j \leq i$ whenever $W_{j} \leq W_{i}$. We now want to work from top to bottom in this partial ordering, splitting off one $W_{i}$ at a time. Since we might not have a maximal element, we use a colimit argument in Theorem 5.3.1. Note that $W_{i}=\bigsqcup_{\bar{x} \in W_{i}} \Gamma[x]$ since whenever two objects of $W_{i}$ are comparable, they have to be isomorphic.

Remark 5.1.5. We work with $\phi$-endomorphisms $g: M \rightarrow M \circ \phi$ of finite free $R \Gamma$-modules $M$, and we know that each finite free $R \Gamma$-module is isomorphic to one of the form $\bigoplus_{b \in B} R \Gamma(?, \beta(b))$ with $B$ finite [Lüc89, Definition 9.17], so we can restrict our attention to $\phi$-endomorphisms of the form

$$
\bigoplus_{b \in B} R \Gamma(?, \beta(b)) \stackrel{g}{\rightarrow} \bigoplus_{b \in B} R \Gamma(\phi(?), \beta(b))
$$


where $B$ is finite. The inclusion of the category of these $\phi$-endomorphisms into the category of all $\phi$-endomorphisms of finite free $R \Gamma$-modules is an equivalence of categories. So the $K_{n}$-groups of these categories are isomorphic, for all $n \in \mathbb{Z}$.

We want to state two preliminary observations that will be important in the sequel. The first one is the following lemma:

Lemma 5.1.6. Let $B_{i}:=\beta^{-1}\left(W_{i}\right)$ for all $W_{i} \in I_{\Gamma}$. Then we have

$$
g\left(\bigoplus_{b \in B_{i}} R \Gamma(?, \beta(b))\right) \subseteq \bigoplus_{j \geq i} \bigoplus_{b \in B_{j}} R \Gamma(\phi(?), \beta(b)) .
$$

Proof. Let $b_{0} \in B_{i}$, and let $v \in R \Gamma\left(?, \beta\left(b_{0}\right)\right)$. Then the following diagram commutes (because $g$ is a natural transformation):

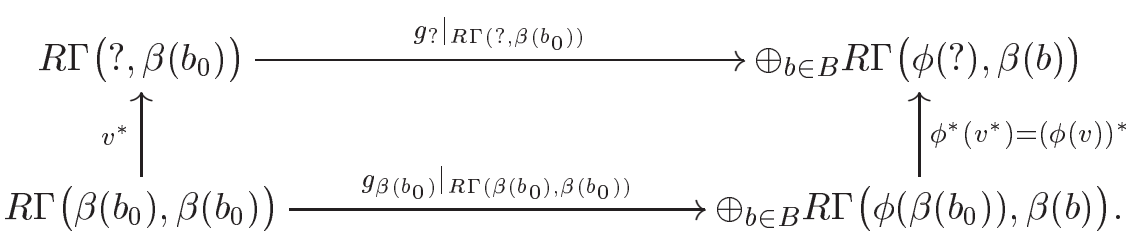

We know that $R \Gamma\left(\phi\left(\beta\left(b_{0}\right)\right), \beta(b)\right)=0$ unless $\phi\left(\beta\left(b_{0}\right)\right) \leq \beta(b)$. But $b_{0} \in B_{i}$ implies $\beta\left(b_{0}\right) \in W_{i}$ and thus also $\phi\left(\beta\left(b_{0}\right)\right) \in W_{i}$, so $\phi\left(\beta\left(b_{0}\right)\right) \leq \beta(b)$ is only possible if $\beta(b) \in W_{j}$ for $j \geq i$. So $R \Gamma\left(\phi\left(\beta\left(b_{0}\right)\right), \beta(b)\right)=0$ if $b \notin B_{j}$ for all $j \geq i$. The map $(\phi(v))^{*}$ respects the direct sum decomposition because it is just precomposition with $\phi(v)$, and thus $g_{?}(v)=(\phi(v))^{*}\left(g_{\beta\left(b_{0}\right)}\left(\operatorname{id}_{\beta\left(b_{0}\right)}\right)\right)$ is nonzero only in components with $b \in B_{j}$ for $j \geq i$. So $g_{\text {? }}\left(R \Gamma\left(\right.\right.$ ?, $\left.\left.\beta\left(b_{0}\right)\right)\right) \subseteq$ $\bigoplus_{j \geq i} \bigoplus_{b \in B_{j}} R \Gamma(\phi(?), \beta(b))$ for all $b_{0} \in B_{i}$, which implies the statement.

Corollary 5.1.7. If $W_{i}$ is the top class, then with $B_{i}:=\beta^{-1}\left(W_{i}\right)$ we have

$$
g\left(\bigoplus_{b \in B_{i}} R \Gamma(?, \beta(b))\right) \subseteq \bigoplus_{b \in B_{i}} R \Gamma(\phi(?), \beta(b)) .
$$

Lemma 5.1.6 implies that if $I_{\Gamma}$ is finite, i.e., $I_{\Gamma}=\left\{W_{1}, \ldots, W_{l}\right\}$ for some $l \in \mathbb{N}$, we can totally order the $W_{i}$ and write $g$ as a block triangular matrix:

$$
\begin{gathered}
\oplus_{b \in B_{l}} R \Gamma(\phi(?), \beta(b)) \\
\vdots \\
\oplus_{b \in B_{1}} R \Gamma(\phi(?), \beta(b))
\end{gathered}
$$

$$
\begin{gathered}
\oplus_{b \in B_{l}} R \Gamma(?, \beta(b)) \\
\left(\begin{array}{ccc}
* & \ldots & \oplus_{b \in B_{1}} R \Gamma(?, \beta(b)) \\
0 & * & \vdots \\
0 & 0 & *
\end{array}\right)
\end{gathered}
$$

This already suggests certain split short exact sequences to us and a splitting in $K_{0}$ looking somewhat like

$$
[g: M \rightarrow M \circ \phi]=\sum_{i=1}^{l}\left[f_{i}: E_{W_{i}} S_{W_{i}} M \rightarrow\left(E_{W_{i}} S_{W_{i}} M\right) \circ \phi\right],
$$


where $E_{W_{i}}$ is to be an "inclusion" of and $S_{W_{i}}$ a "projection" onto the relevant component, but we will do all of this in detail in Sections 5.2 and 5.3.

The second preliminary observation is the following lemma:

Lemma 5.1.8. If $h: M \rightarrow M^{\prime}$ is a natural transformation of finite free $R \Gamma$-modules, with $M=\bigoplus_{b \in B} R \Gamma(?, \beta(b))$ and $M^{\prime}=\bigoplus_{b^{\prime} \in B^{\prime}} R \Gamma\left(?, \beta^{\prime}\left(b^{\prime}\right)\right)$, then with $B_{i}:=\beta^{-1}\left(W_{i}\right)$ we have

$$
h\left(\bigoplus_{b \in B_{i}} R \Gamma(?, \beta(b))\right) \subseteq \bigoplus_{j \geq i} \bigoplus_{b^{\prime} \in B_{j}^{\prime}} R \Gamma\left(?, \beta^{\prime}\left(b^{\prime}\right)\right) .
$$

Proof. This is analogous to the proof of Lemma 5.1.6. Let $b_{0} \in B_{i}$ and let $v \in R \Gamma\left(?, \beta\left(b_{0}\right)\right)$. The following diagram commutes:

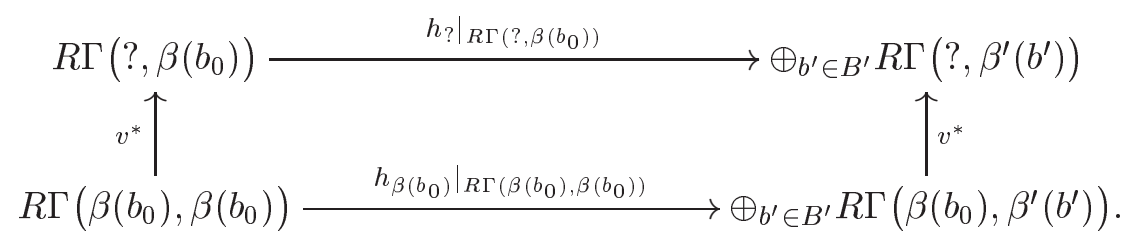

We again have $R \Gamma\left(\beta\left(b_{0}\right), \beta^{\prime}\left(b^{\prime}\right)\right)=0$ unless $\beta\left(b_{0}\right) \leq \beta^{\prime}\left(b^{\prime}\right)$. Since $\beta\left(b_{0}\right) \in W_{i}$, this implies $R \Gamma\left(\beta\left(b_{0}\right), \beta^{\prime}\left(b^{\prime}\right)\right)=0$ if $\beta^{\prime}\left(b^{\prime}\right) \notin W_{j}$ for all $j \geq i$, which is equivalent to $b^{\prime} \notin B_{j}^{\prime}$ for all $j \geq i$. Thus again $h_{\text {? }}(v)$ is nonzero only in components for which $b^{\prime} \in B_{j}^{\prime}$, for some $j \geq i$.

Corollary 5.1.9. If $h: M \rightarrow M^{\prime}$ is a natural transformation of finite free $R \Gamma$-modules, with $M=\bigoplus_{b \in B} R \Gamma(?, \beta(b))$ and $M^{\prime}=\bigoplus_{b^{\prime} \in B^{\prime}} R \Gamma\left(?, \beta^{\prime}\left(b^{\prime}\right)\right)$, if $W_{i}$ is the top class, $B_{i}:=\beta^{-1}\left(W_{i}\right)$ and $B_{i}^{\prime}:=\beta^{\prime-1}\left(W_{i}\right)$, then

$$
h\left(\bigoplus_{b \in B_{i}} R \Gamma(?, \beta(b))\right) \subseteq \bigoplus_{b^{\prime} \in B_{i}^{\prime}} R \Gamma\left(?, \beta^{\prime}\left(b^{\prime}\right)\right) .
$$

\subsection{Definition of the Splitting Functors}

Now we are ready to define the splitting functors. We define functors $E$ and $S$ as variations of the restriction functor $E$ and splitting functor $S$ defined in Definition 1.2.10. We denote by $W_{i}$ not only a subset of Is $\Gamma$, but also the full subcategory of $\Gamma$ containing all $x \in \Gamma$ with $\bar{x} \in W_{i}$. We obtain an endomorphism $\phi_{i}:=\left.\phi\right|_{W_{i}}: W_{i} \rightarrow W_{i}$ by restricting $\phi$ to $W_{i}$. The inclusion map $\operatorname{incl}_{i}: W_{i} \rightarrow \Gamma$ is a map of categories with endofunctors, $\phi \circ$ incl $_{i}=$ incl $_{i} \circ \phi_{i}$.

Definition 5.2.1. We define the extension functor $E_{W_{i}}$ by

$$
E_{W_{i}}:=\left(\operatorname{incl}_{i}\right)_{*}: \phi_{i}-\operatorname{end}_{\mathrm{ff} R W_{i}} \rightarrow \phi-\operatorname{end}_{\mathrm{ffR} \Gamma} .
$$


The extension functor $E_{x}$ preserves the property of a module to be finitely generated free as stated in Remark 1.2.11, so $E_{W_{i}}$ also does and is well-defined. The functor $E_{x}$ also preserves split exact sequences and thus exact sequences of finitely generated free modules, and so $E_{W_{i}}$ preserves exact sequences and induces a well-defined map in $K$-theory.

We also want to define a splitting functor

$$
S_{W_{i}}: \phi-\operatorname{end}_{\mathrm{ff} R \Gamma} \rightarrow \phi_{i} \text {-end }{\mathrm{ff} R W_{i}},
$$

but this is a little more complicated. On a module $M \in \mathrm{ff} R \Gamma$, the splitting functor $S_{W_{i}} M$ should be $\bigoplus_{\bar{x} \in W_{i}} S_{\bar{x}} M$. We can equivalently define $S_{W_{i}}$ on a module $M \in$ ff $R \Gamma$ by $S_{W_{i}}(M):=M \otimes_{R \Gamma} B$, where $B$ is the $R \Gamma-R W_{i^{-}}$ bimodule given by

$$
B(x, y)= \begin{cases}R \Gamma\left(\operatorname{incl}_{i}(x), y\right) & y \in W_{i} \subseteq \Gamma, \\ \{0\} & y \notin W_{i},\end{cases}
$$

where $x \in \mathrm{Ob} W_{i}$ and $y \in \mathrm{Ob} \Gamma$. On morphisms, the definition is a little more awkward; once again, we have to take into account the difference between $S_{W_{i}}(M \circ \phi)$ and $\left(S_{W_{i}} M\right) \circ \phi_{i}$.

We define $S_{W_{i}}(M \stackrel{g}{\rightarrow} M \circ \phi)$ as the following composition:

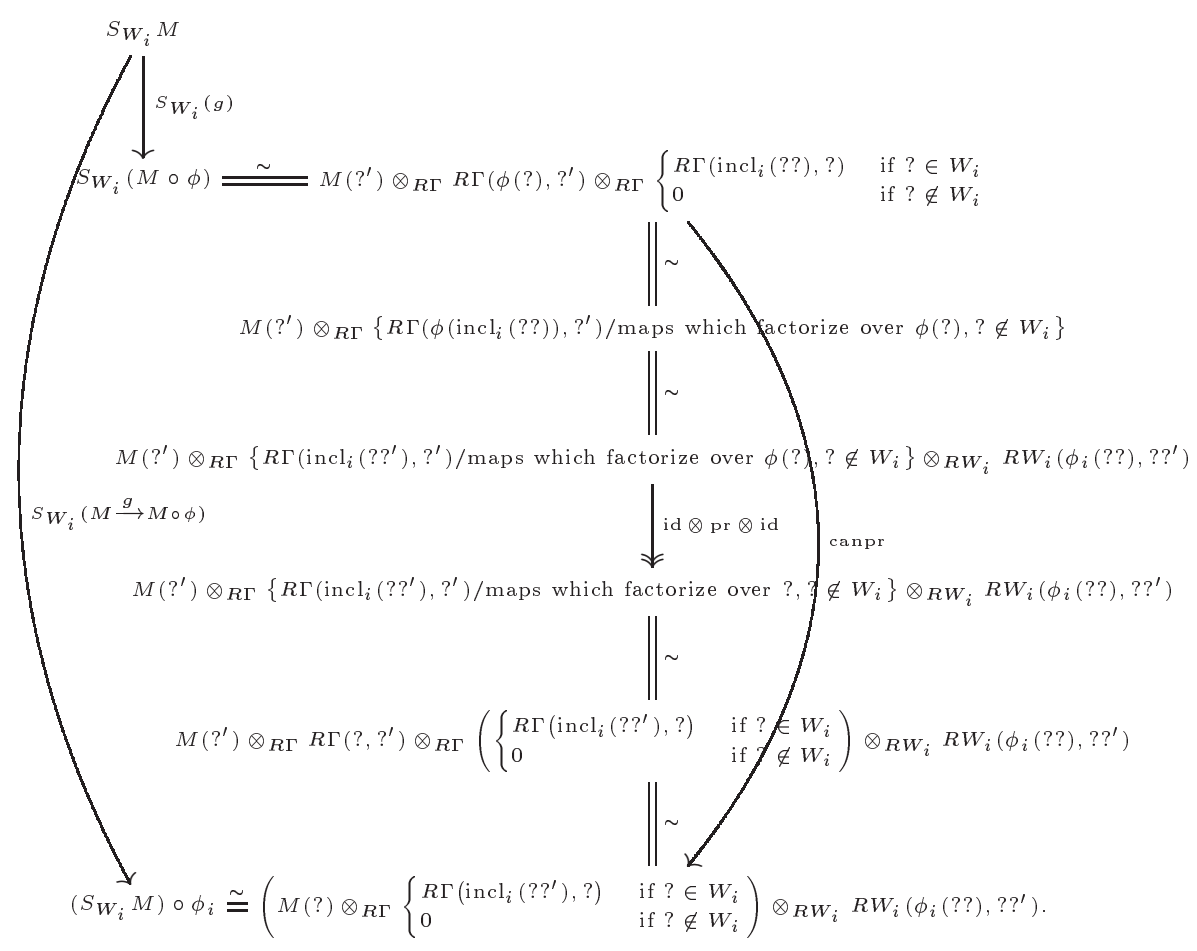

Note that $? \in W_{i}$ implies $\phi(?) \in W_{i}$, so the projection pr is well-defined. Note further that the projection pr is an isomorphism if $\left.\phi\right|_{W_{i}}: W_{i} \rightarrow W_{i}$ is 
surjective. (In the language of Section 5.3 this means $Q_{W_{i}}=\emptyset$. So restricted to $T_{W_{i}}$ the projection $\mathrm{pr}$ is an isomorphism.)

Example 5.2.2. If $W_{i}$ is the top class, no maps from $\operatorname{incl}_{i}($ ??') can factor over anything outside $W_{i}$, so nothing is divided out and canpr is an isomorphism. (If $W_{i}$ is the top class, $S_{W_{i}}=\operatorname{res}_{W_{i}}$, and $\operatorname{res}_{W_{i}}$ and $\operatorname{res}_{\phi}$ commute.)

Example 5.2.3. The map canpr is not always an isomorphism: Consider the category $\Gamma$ with three objects $\{a, b, c\}$ that has exactly one nontrivial morphism, $v: b \rightarrow c$. Take the endofunctor $\phi: \Gamma \rightarrow \Gamma, a \mapsto b, b \mapsto b, c \mapsto$ $c, v \mapsto v$. Then $\Gamma$ splits up into two $\phi$-invariant subcategories, $W_{i}$ with the objects $a$ and $b$ and $W_{j}$ with the object $c$. If we look at the $R \Gamma$-module $M: \Gamma \rightarrow R$-Mod which is $R$ at every object and $\operatorname{id}_{R}$ at every morphism, then

$$
S_{i}(M \circ \phi)(a)=\operatorname{res}_{a}(M \circ \phi)=M \circ \phi(a)=M(b)=R,
$$

whereas

$$
\left(S_{i} M\right) \circ \phi_{i}(a)=S_{i} M(b)=M(b) / \operatorname{Im} M(v)=0 .
$$

The construction of $S_{W_{i}}$ is functorial in maps $h:(M \stackrel{g}{\rightarrow} M \circ \phi) \rightarrow\left(N \stackrel{g^{\prime}}{\rightarrow}\right.$ $N \circ \phi)$ : Knowing that the diagram

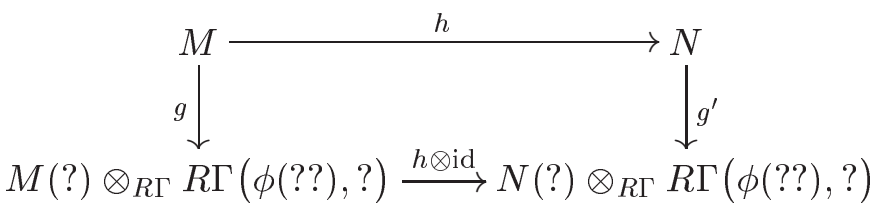

commutes, we can apply $S_{W_{i}}$ to this diagram and compose the vertical maps with canpr. $\left(S_{W_{i}}\right.$ on the morphisms is tensoring with id ${ }_{B}$, i.e., $S_{W_{i}}(h):=$ $h \otimes \operatorname{id}_{B}$. The map canpr does not touch the left hand factor and maps the identity to the identity.) We obtain

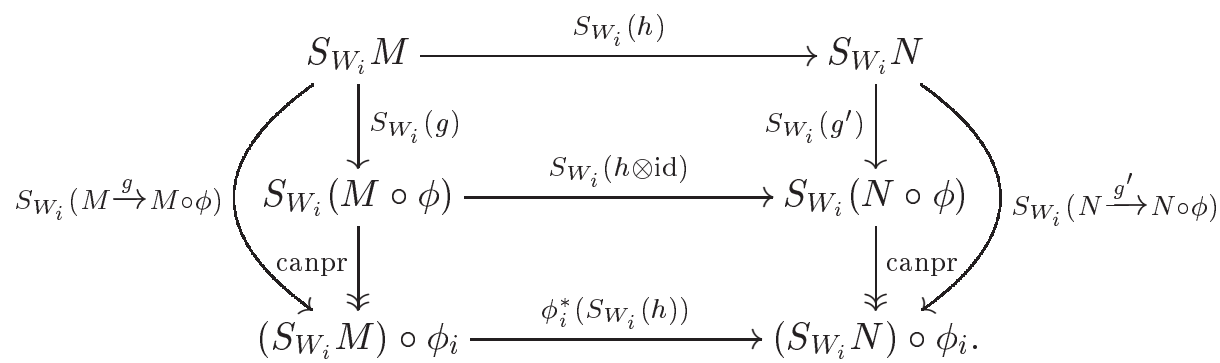

So we can make the following definition.

Definition 5.2.4. We define the splitting functor $S_{W_{i}}$ to be

$$
\begin{aligned}
S_{W_{i}}: \quad \text {-end }_{\mathrm{ff} R \Gamma} & \rightarrow \phi_{i} \text {-end } \mathrm{enf}_{\mathrm{f} W_{i}} \\
(M \stackrel{g}{\rightarrow} M \circ \phi) & \mapsto S_{W_{i}}(M \stackrel{g}{\rightarrow} M \circ \phi):=\operatorname{canpr} \circ S_{W_{i}}(g) .
\end{aligned}
$$


The splitting functor $S_{x}$ sends finitely generated free modules to finitely generated free modules and it also preserves split exact sequences, so exact sequences of finitely generated free modules. So the splitting functor $S_{W_{i}}$ is well defined and induces a splitting map on the level of $K$-theory. We are mostly interested in $K_{0}$ since that is where our invariant lives. But as stated in Remark 2.1.5, we can treat all higher $K$-groups since $\phi$-end $\mathrm{eff}_{\mathrm{f}}$ is an exact category. So analogously to [Lüc89] we let $K$ stand for any $K_{n}, n \in \mathbb{Z}$, and define the splitting functors for all algebraic $K$-groups simultaneously. We obtain maps

$$
K\left(S_{W_{i}}\right): K\left(\phi-\operatorname{end}_{\mathrm{ff} R \Gamma}\right) \rightarrow K\left(\phi_{i}-\operatorname{end}_{\mathrm{ff} R W_{i}}\right) .
$$

Remember that we had obtained the category $\phi$-end $\mathrm{d}_{\mathrm{ff} R \Gamma}$ from the category with endofunctor $(\Gamma, \phi)$. We combined all categories with endofunctor in the category Endo(EI-Cat) which was defined in Definition 2.2.1. We introduce the full subcategory of Endo(EI-Cat) where the endofunctors are orderrespecting.

Definition 5.2.5. Let Endo(EI-Cat) ord be the category of categories $(\Gamma, \phi)$ with order-respecting endofunctor $\phi$.

We can now ask ourselves whether the application

$$
\begin{aligned}
\text { Split } \left.K: \operatorname{Ob}(\text { Endo(EI-Cat) })_{\text {ord }}\right) & \rightarrow \mathcal{A} b \\
(\Gamma, \phi) & \mapsto \oplus_{W_{i} \in I_{\Gamma}} K\left(\phi_{i}-\text { end }_{\mathrm{ff} R W_{i}}\right)
\end{aligned}
$$

extends to a functor from Endo(EI-Cat) ord to $\mathcal{A} b$.

Note that any functor of categories with endofunctors $F:\left(\Gamma_{1}, \phi\right) \rightarrow$ $\left(\Gamma_{2}, \psi\right)$, i.e., $F: \Gamma_{1} \rightarrow \Gamma_{2}$ such that $\psi \circ F=F \circ \phi$, preserves the decomposition of $\Gamma_{1}$ into $\left\{W_{i}\right\}_{W_{i} \in I_{\Gamma_{1}}}$ and of $\Gamma_{2}$ into $\left\{V_{j}\right\}_{V_{j} \in I_{\Gamma_{2}}}$ : If $x, x^{\prime} \in W_{i}$, then there are $n, n^{\prime}$ such that $\overline{\phi^{n}(x)}=\overline{\phi^{n^{\prime}}\left(x^{\prime}\right)} \in W_{i}$. Let $j(i)$ be the index such that $\overline{F\left(\phi^{n}(x)\right)}=\overline{F\left(\phi^{n^{\prime}}\left(x^{\prime}\right)\right)} \in V_{j(i)}$. Then $\psi^{n}(F(x))=F\left(\phi^{n}(x)\right) \in V_{j(i)}$ and $\psi^{n^{\prime}}\left(F\left(x^{\prime}\right)\right)=F\left(\phi^{n^{\prime}}\left(x^{\prime}\right)\right) \in V_{j(i)}$, so $F(x)$ and $F\left(x^{\prime}\right)$ are both in $V_{j(i)}$. This way we can define a (well-defined) map $j: I_{\Gamma_{1}} \rightarrow I_{\Gamma_{2}}, i \mapsto j(i)$. We can define restricted functors $F_{W_{i}}: W_{i} \rightarrow V_{j(i)}$, and we have $\operatorname{incl}_{j(i)} \circ F_{W_{i}} \circ \phi_{i}=$ $\left.(F \circ \phi)\right|_{W_{i}}=\left.(\psi \circ F)\right|_{W_{i}}=\operatorname{incl}_{j(i)} \circ \psi_{j(i)} \circ F_{W_{i}}$, so $F_{W_{i}}$ is a functor between categories with endofunctors $\left(W_{i}, \phi_{i}\right)$ and $\left(V_{j(i)}, \psi_{j(i)}\right)$.

Given a functor $F:\left(\Gamma_{1}, \phi\right) \rightarrow\left(\Gamma_{2}, \psi\right)$ of categories with order-respecting endofunctors, we define Split $K(F)$ by

$$
\begin{aligned}
& \operatorname{Split} K(F):= \\
& \bigoplus_{W_{i} \in I_{\Gamma_{1}}} \operatorname{inclus}_{j(i)} K\left(F_{W_{i *}}\right): \bigoplus_{W_{i} \in I_{\Gamma_{1}}} K\left(\phi_{i}-\operatorname{end}_{\mathrm{ff} R W_{i}}\right) \rightarrow \bigoplus_{V_{j} \in I_{\Gamma_{2}}} K\left(\psi_{j}-\operatorname{end}_{\mathrm{ff} R V_{j}}\right),
\end{aligned}
$$




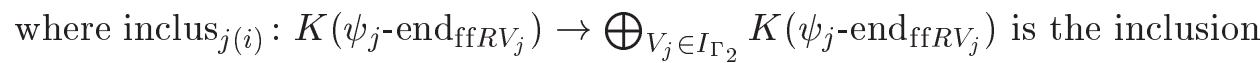
of the summand into the direct sum. The map Split $K$ is indeed a functor on $\operatorname{Endo}(\mathrm{EI}-\mathrm{Cat})_{\text {ord }}$ :

$$
\begin{aligned}
\operatorname{Split} K\left(\operatorname{Id}_{(\Gamma, \phi)}\right) & =\bigoplus_{W_{i} \in I_{\Gamma}} \operatorname{inclus}_{i} K\left(\operatorname{Id}_{\left(W_{i}, \phi_{i}\right)_{*}}\right) \\
& =\operatorname{Id}_{\bigoplus_{W_{i} \in I_{\Gamma}} K\left(\phi_{i}-\text { end }_{f f R W_{i}}\right)}
\end{aligned}
$$

Split $K(F) \circ \operatorname{Split} K(G)$

$$
\begin{aligned}
& =\left(\bigoplus_{V_{j} \in I_{\Gamma_{2}}} \operatorname{inclus}_{k(j)} K\left(F_{V_{j} *}\right)\right) \circ\left(\bigoplus_{W_{i} \in I_{\Gamma_{1}}} \operatorname{inclus}_{j(i)} K\left(G_{W_{i} *}\right)\right) \\
& =\bigoplus_{W_{i} \in I_{\Gamma_{1}}} \operatorname{inclus}_{k(j(i))} \underbrace{K\left(F_{V_{j(i)}}\right) \circ K\left(G_{W_{i} *}\right)}_{=K\left(F_{V_{j(i)}} \circ G_{W_{i} *}\right)=K\left((F G)_{W_{i}}\right)} \\
& =\bigoplus_{W_{i} \in I_{\Gamma_{1}}} \operatorname{inclus}_{k(j(i))} K\left((F G)_{W_{i} *}\right) \\
& =\operatorname{Split} K(F \circ G) .
\end{aligned}
$$

So the following definition makes sense:

Definition 5.2.6. We define the splitting functor Split $K$ to be

$$
\begin{aligned}
\text { Split } K: \text { Endo(EI-Cat) } & \rightarrow \mathcal{A r d} \\
(\Gamma, \phi) & \mapsto \oplus_{W_{i} \in I_{\Gamma}} K\left(\phi_{i} \text {-end }{\text { ff } R W_{i}}_{i}\right) \\
F & \mapsto \operatorname{Split} K(F) .
\end{aligned}
$$

The functors $E_{W_{i}}$ and $S_{W_{i}}$ induce maps

$$
\begin{aligned}
& K\left(E_{W_{i}}\right): K\left(\phi_{i}-\operatorname{end}_{\mathrm{ff} R W_{i}}\right) \rightarrow K\left(\phi-\operatorname{end}_{\mathrm{ff} R \Gamma}\right) \\
& \text { and } K\left(S_{W_{i}}\right): K\left(\phi-\operatorname{end}_{\mathrm{ff} R \Gamma}\right) \rightarrow K\left(\phi_{i} \text {-end } \mathrm{enfRW}_{i}\right) \text {. }
\end{aligned}
$$

Taking the direct sum over the $W_{i} \in I_{\Gamma}$ gives a group homomorphism

$$
E_{\Gamma, \phi}:=\bigoplus_{W_{i} \in I_{\Gamma}} K\left(E_{W_{i}}\right): \bigoplus_{W_{i} \in I_{\Gamma}} K\left(\phi_{i}-\operatorname{end}_{\mathrm{ff} R W_{i}}\right) \rightarrow K\left(\phi-\operatorname{end}_{\mathrm{ff} R \Gamma}\right)
$$

for every $(\Gamma, \phi) \in$ Endo(EI-Cat) ${ }_{\text {ord }}$. Defining

$$
\begin{aligned}
K: \text { Endo(EI-Cat) } & \rightarrow \mathcal{A r d} \\
(\Gamma, \phi) & \mapsto K\left(\phi-\operatorname{end}_{\mathrm{ff} R W}\right) \\
F & \mapsto K\left(F_{*}\right)
\end{aligned}
$$

to have compatible notation, we indeed obtain a natural transformation

$$
E: \text { Split } K \rightarrow K
$$


of functors from Endo(EI-Cat) ord to $\mathcal{A} b$ because if $F:\left(\Gamma_{1}, \phi\right) \rightarrow\left(\Gamma_{2}, \psi\right)$ is a morphism we have a commutative diagram

$$
\begin{aligned}
& \oplus_{W_{i} \in I_{\Gamma_{1}}} K\left(\phi_{i}-\operatorname{end}_{\mathrm{ffR} W_{i}}\right) \longrightarrow K\left(\phi-\operatorname{end}_{\mathrm{ff} R \Gamma_{1}}\right) \\
& \oplus_{W_{i} \in I_{\Gamma_{1}}} \operatorname{inclus}_{j(i)} K\left(F_{W_{i *}} \downarrow \downarrow \quad E_{\Gamma_{2}, \psi} \downarrow \downarrow K\left(F_{*}\right)\right. \\
& \oplus_{V_{j} \in I_{\Gamma_{2}}} K\left(\psi_{j} \text {-end } \mathrm{ffR}_{j}\right) \longrightarrow K\left(\psi-E_{\Gamma_{2}, \psi} \longrightarrow \operatorname{end}_{\mathrm{ff} R \Gamma_{2}}\right) .
\end{aligned}
$$

Commutativity is seen as follows: By definition of $F_{W_{i}}$, we have the equation $\operatorname{incl}_{j(i)} \circ F_{W_{i}}=F \circ \operatorname{incl}_{i}$, and so $K\left(E_{j(i)}\right) \circ K\left(F_{W_{i *}}\right)=K\left(\operatorname{incl}_{j(i)_{*}}\right) \circ$ $K\left(F_{W_{i *}}\right)=K\left(F_{*}\right) \circ K\left(\operatorname{incl}_{i *}\right)=K\left(F_{*}\right) \circ K\left(E_{W_{i}}\right)$ which implies

$$
\begin{aligned}
& \left(\bigoplus_{V_{j} \in I_{\Gamma_{2}}} K\left(E_{V_{j}}\right)\right) \circ\left(\bigoplus_{W_{i} \in I_{\Gamma_{1}}} \operatorname{inclus}_{j(i)} K\left(F_{W_{i *}}\right)\right) \\
& =\bigoplus_{W_{i} \in I_{\Gamma_{1}}} K\left(E_{j(i)}\right) \circ K\left(F_{W_{i *}}\right) \\
& =\bigoplus_{W_{i} \in I_{\Gamma_{1}}} K\left(F_{*}\right) \circ K\left(E_{W_{i}}\right) \\
& =K\left(F_{*}\right) \circ\left(\bigoplus_{W_{i} \in I_{\Gamma_{1}}} K\left(E_{W_{i}}\right)\right) .
\end{aligned}
$$

Finite coproducts and finite products agree in the category of abelian groups, so for any finite subset $I_{\Gamma}^{\prime} \subseteq I_{\Gamma}$ we get a homomorphism

$$
S_{\Gamma, \phi, I_{\Gamma}^{\prime}}:=\prod_{W_{i} \in I_{\Gamma}^{\prime}} K\left(S_{W_{i}}\right): K\left(\phi-\operatorname{end}_{\mathrm{ff} R \Gamma}\right) \rightarrow \bigoplus_{W_{i} \in I_{\Gamma}^{\prime}} K\left(\phi_{i}-\operatorname{end}_{\mathrm{ff} R W_{i}}\right) .
$$

We set

$$
S_{\Gamma, \phi}:=\operatorname{colim}_{I_{\Gamma}^{\prime} \subseteq I_{\Gamma}} S_{\Gamma, \phi, I_{\Gamma}^{\prime}}: K\left(\phi-\operatorname{end}_{\mathrm{ff} R \Gamma}\right) \rightarrow \operatorname{Split} K\left(\phi-\operatorname{end}_{\mathrm{ff} R \Gamma}\right) .
$$

This is our desired splitting morphism.

These splitting morphisms $S_{\Gamma, \phi}$ actually combine to form a natural transformation

$$
S: K \rightarrow \text { Split } K
$$

of functors from Endo(EI-Cat) ord to $\mathcal{A} b$, but we can show this best using the proof of the next theorem, Theorem 5.3.1. There we see that ES is naturally equivalent to Id and $S E$ is naturally equivalent to Id, thereby for a functor $F:\left(\Gamma_{1}, \phi\right) \rightarrow\left(\Gamma_{2}, \psi\right)$ we obtain $S F=S F E S=S E F S=F S$.

We introduce the same notation for $E$. (Here it is of course not necessary since $E$ is defined on the direct sum already. But we want to use this notation in the proof of Theorem 5.3.1.) For finite $I_{\Gamma}^{\prime} \subseteq I_{\Gamma}$ let

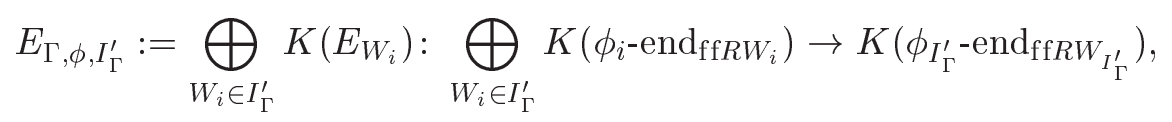


where $\phi_{I_{\Gamma}^{\prime}-e^{-}} d_{f f R W_{I_{\Gamma}^{\prime}}}$ is the full subcategory of $\phi$-end $\mathrm{fff}_{\mathrm{f} \Gamma}$ with objects $\phi_{I_{\Gamma}^{\prime}}$ endomorphisms of finite free $R W_{I_{\Gamma}^{\prime}}$-modules, where $W_{I_{\Gamma}^{\prime}} \subset \Gamma$ is the full subcategory of $\Gamma$ with objects in $\cup_{W_{i} \in I_{\Gamma}^{\prime}} W_{i}$. Then $E_{\Gamma, \phi}=\operatorname{colim}_{I_{\Gamma}^{\prime} \subseteq I_{\Gamma}} E_{\Gamma, \phi, I_{\Gamma}^{\prime}}$.

\subsection{From Categories to Groupoids}

Now we are ready to prove the splitting results. We state in analogy to [Lüc89, Theorem 10.34] that there is a natural equivalence between $K$ and Split $K$ given by $E$ and $S$.

Theorem 5.3.1. We have inverse pairs of natural equivalences $E$ and $S$ between the functors

$$
K \text { and Split } K: \text { Endo(EI-Cat) })_{\text {ord }} \rightarrow \mathcal{A} b \text {. }
$$

I.e., let $(\Gamma, \phi)$ be an EI-category with order-respecting endofunctor $\phi$, then

$$
K\left(\phi-\operatorname{end}_{\mathrm{ff} R \Gamma}\right) \cong \operatorname{Split} K\left(\phi-\operatorname{end}_{\mathrm{ff} R \Gamma}\right),
$$

where the isomorphism is given by $S_{\Gamma, \phi}$ with inverse $E_{\Gamma, \phi}$ and is natural in $(\Gamma, \phi)$.

Proof. We see that

$$
S_{\Gamma, \phi} \circ E_{\Gamma, \phi}=\operatorname{colim}_{I_{\Gamma}^{\prime}} S_{\Gamma, \phi, I_{\Gamma}^{\prime}} \circ \operatorname{colim}_{I_{\Gamma}^{\prime}} E_{\Gamma, \phi, I_{\Gamma}^{\prime}}=\operatorname{colim}_{I_{\Gamma}^{\prime}}\left(S_{\Gamma, \phi, I_{\Gamma}^{\prime}} \circ E_{\Gamma, \phi, I_{\Gamma}^{\prime}}\right)
$$

and

$$
E_{\Gamma, \phi} \circ S_{\Gamma, \phi}=\operatorname{colim}_{I_{\Gamma}^{\prime}} E_{\Gamma, \phi, I_{\Gamma}^{\prime}} \circ \operatorname{colim}_{I_{\Gamma}^{\prime}} S_{\Gamma, \phi, I_{\Gamma}^{\prime}}=\operatorname{colim}_{I_{\Gamma}^{\prime}}\left(E_{\Gamma, \phi, I_{\Gamma}^{\prime}} \circ S_{\Gamma, \phi, I_{\Gamma}^{\prime}}\right) .
$$

Hence it suffices to show for any finite $I_{\Gamma}^{\prime} \subseteq I_{\Gamma}$ that $E_{\Gamma, \phi, I_{\Gamma}^{\prime}} \circ S_{\Gamma, \phi, I_{\Gamma}^{\prime}}=\mathrm{Id}$ and $S_{\Gamma, \phi, I_{\Gamma}^{\prime}} \circ E_{\Gamma, \phi, I_{\Gamma}^{\prime}}=$ Id hold.

1) We show that $S_{\Gamma, \phi, I_{\Gamma}^{\prime}} \circ E_{\Gamma, \phi, I_{\Gamma}^{\prime}}=\mathrm{Id}$.

$$
\begin{aligned}
S_{\Gamma, \phi, I_{\Gamma}^{\prime}} \circ E_{\Gamma, \phi, I_{\Gamma}^{\prime}} & =\left(\bigoplus_{W_{i} \in I_{\Gamma}^{\prime}} K\left(S_{W_{i}}\right)\right) \circ\left(\bigoplus_{V_{j} \in I_{\Gamma}^{\prime}} K\left(E_{V_{j}}\right)\right) \\
& =\bigoplus_{W_{i} \in I_{\Gamma}^{\prime}} \bigoplus_{V_{j} \in I_{\Gamma}^{\prime}} K\left(S_{W_{i}} E_{V_{j}}\right) \\
& =\bigoplus_{W_{i} \in I_{\Gamma}^{\prime}} K\left(S_{W_{i}} E_{W_{i}}\right)
\end{aligned}
$$

since if $i \neq j$ the corresponding modules are already 0 [Lüc89, Lemma 9.31]. Thus we need to show that $K\left(S_{W_{i}} E_{W_{i}}\right)=\operatorname{Id}_{K\left(\phi_{i} \text {-end } \operatorname{enf}_{\mathrm{ff}}\right)}$ for all $W_{i} \in I_{\Gamma}^{\prime}$. We know that $S_{W_{i}} E_{W_{i}}$ : ff $R W_{i} \rightarrow$ ff $R W_{i}$ is naturally equivalent to the 
identity functor [Lüc89, Lemma 9.31]. This natural equivalence is given explicitly by

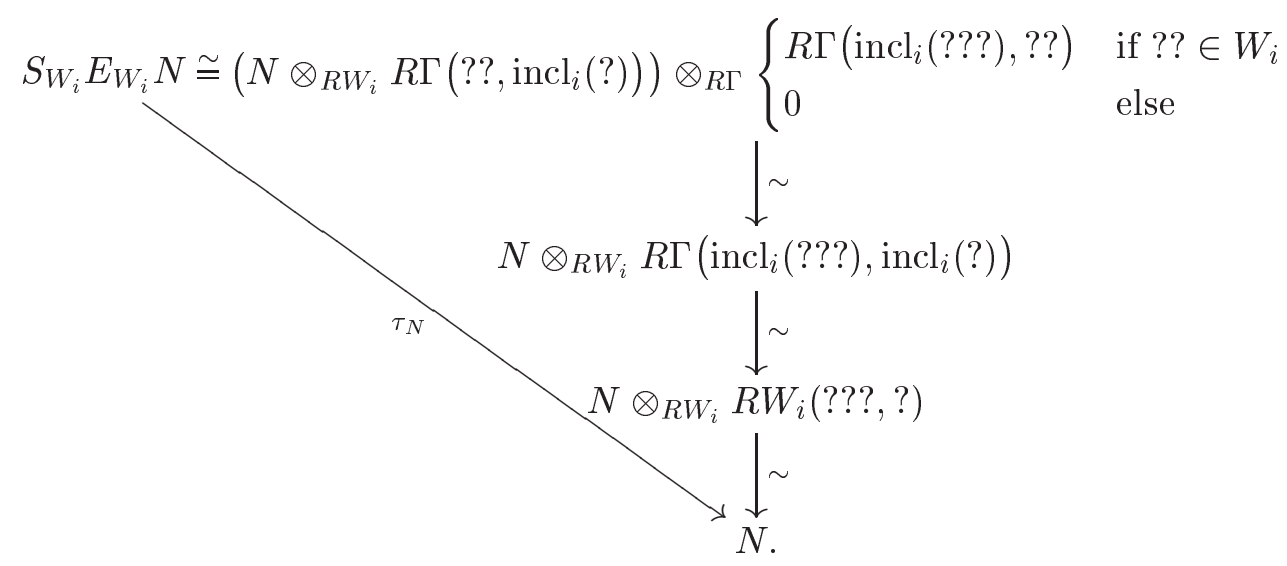

The first vertical map is an isomorphism since no morphism of objects in $W_{i}$ can factor through an object outside $W_{i}$, the second vertical map is an isomorphism since $W_{i} \subseteq \Gamma$ is a full subcategory. Thus on $\phi_{i}$-end $\mathrm{ff}_{R W_{i}}$ we have

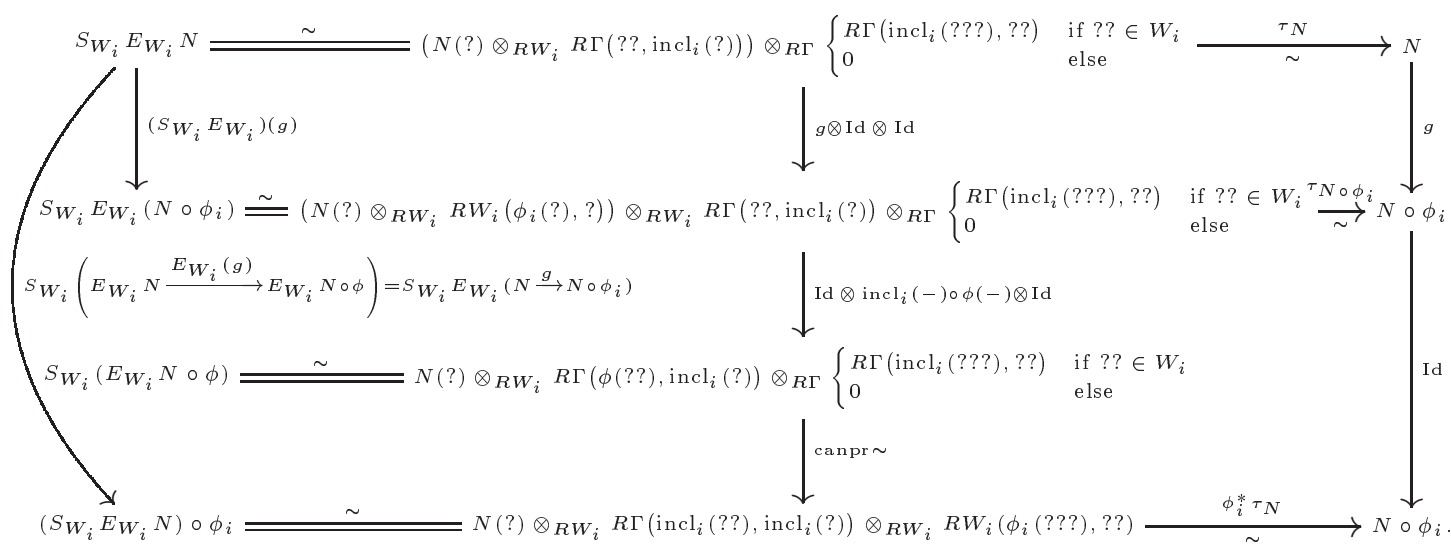

The map canpr is an isomorphism since no maps in $R \Gamma\left(\phi\left(\operatorname{incl}_{i}(? ? ?)\right), \operatorname{incl}_{i}(?)\right)$

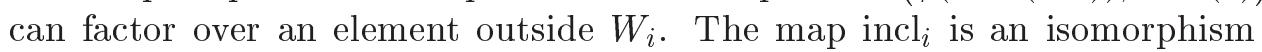
since $W_{i}$ is a full subcategory. So if $\phi$ is an isomorphism, the middle vertical arrow is.

The functor $\tau$ is a natural transformation between $S_{W_{i}} E_{W_{i}}$ and Id since the above map on objects commutes with morphisms: If we have a morphism $h:\left(N \stackrel{g}{\rightarrow} N \circ \phi_{i}\right) \rightarrow\left(N^{\prime} \stackrel{g^{\prime}}{\rightarrow} N^{\prime} \circ \phi_{i}\right)$, then in the above construction $h$ only touches the first factor, canpr $\circ\left(\mathrm{id} \otimes \operatorname{incl}_{i}(-) \circ \phi(-) \otimes \mathrm{id}\right)$ only the rest. So $\tau$ is a natural equivalence between $S_{W_{i}} E_{W_{i}}$ and Id as functors on $\phi_{i}$-end $\mathrm{fff}_{\mathrm{f} W_{i}}$. We conclude that

$$
K\left(S_{W_{i}} E_{W_{i}}\right)=K\left(\operatorname{Id}_{\phi_{i}-\operatorname{end}_{\mathrm{ff} R W_{i}}}\right)=\operatorname{Id}_{K\left(\phi_{i}-\operatorname{end}_{\mathrm{ff} R W_{i}}\right)}
$$


2) We show that $E_{\Gamma, \phi, I_{\Gamma}^{\prime}} \circ S_{\Gamma, \phi, I_{\Gamma}^{\prime}}=\mathrm{Id}$.

The proof proceeds inductively over the cardinality of $I_{\Gamma}^{\prime}$. The beginning $I_{\Gamma}^{\prime}=\emptyset$ is trivial. In the induction step, choose $W_{i}$ maximal in $\left\{W_{i}\right\}_{W_{i} \in I_{\Gamma}^{\prime}}$ and write $I_{\Gamma}^{\prime \prime}=I_{\Gamma}^{\prime} \backslash\{i\}$. (This is possible since $I_{\Gamma}^{\prime}$ is finite.) We want to show that we have a cofibration sequence of functors on $\phi_{I_{\Gamma}^{\prime}}$-end $\mathrm{dff}_{\mathrm{f} R W_{I_{\Gamma}^{\prime}}}$

$$
0 \rightarrow E_{W_{i}} S_{W_{i}} \rightarrow \mathrm{Id} \rightarrow F G \rightarrow 0,
$$

where $W_{I_{\Gamma}^{\prime}} \subseteq \Gamma$ is the full subcategory of $\Gamma$ with objects in $\bigcup_{W_{i} \in I_{\Gamma}^{\prime}} W_{i}$ and $F$ and $G$ remain to be explained. By Remark 5.1.5, we can restrict ourselves to modules of the form

$$
M=\bigoplus_{b \in B} R W_{I_{\Gamma}^{\prime}}(?, \beta(b)) .
$$

Then $S_{W_{j}} M \cong \bigoplus_{b \in B_{j}} R W_{j}(?, \beta(b))$ for all $W_{j} \in I_{\Gamma}^{\prime}$ and $E_{W_{j}} S_{W_{j}} M \cong$ $\bigoplus_{b \in B_{j}} R W_{I_{\Gamma}^{\prime}}(?, \beta(b))$ for all $W_{j} \in I_{\Gamma}^{\prime}$ [Lüc89, Lemma 9.31]. Under the above isomorphism, the element $S_{W_{j}}\left(M \stackrel{g}{\rightarrow} M \circ \phi_{I_{\Gamma}^{\prime}}\right)$ is nothing but the map $\left.\operatorname{pr}_{\oplus_{b \in B_{j}}} R W_{I_{\Gamma}^{\prime}}\left(\operatorname{incl}_{j}\left(\phi_{j}(?)\right), \beta(b)\right) \circ\right|_{\oplus_{b \in B_{j}} R W_{I_{\Gamma}^{\prime}}\left(\operatorname{incl}_{j}(?), \beta(b)\right)}$ and the element given

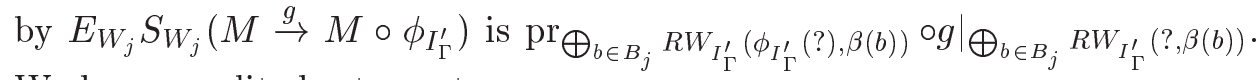
We have a split short exact sequence

$$
0 \rightarrow \bigoplus_{b \in B_{i}} R W_{I_{\Gamma}^{\prime}}(?, \beta(b)) \rightarrow \bigoplus_{b \in B} R W_{I_{\Gamma}^{\prime}}(?, \beta(b)) \rightarrow \bigoplus_{b \in B \backslash B_{i}} R W_{I_{\Gamma}^{\prime}}(?, \beta(b)) \rightarrow 0 .
$$

By Corollary 5.1.7 we know that the diagram

$$
\begin{gathered}
\oplus_{b \in B_{i}} R W_{I_{\Gamma}^{\prime}}(?, \beta(b)) \longrightarrow \text { incl } \\
\operatorname{pr}_{\oplus_{b} \in B_{i}} R W_{I_{\Gamma}^{\prime}}\left(\phi_{I_{\Gamma}^{\prime}}(?), \beta(b)\right) \\
\left.\oplus_{b \in B_{i}} R W_{I_{\Gamma}^{\prime}}\left(\phi_{I_{\Gamma}^{\prime}}(?), \beta(b)\right) \longrightarrow\right|_{\oplus_{b \in B_{i}}} R W_{I_{\Gamma}^{\prime}(?, \beta(b))} R W_{I_{\Gamma}^{\prime}}(?, \beta(b))
\end{gathered}
$$

commutes since $W_{i}$ is the top class. This is the specialty of the top class, that is why we have to split off one $W_{j}$ at a time. We define functors

$$
\begin{aligned}
& G: \quad \text { ff } R W_{I_{\Gamma}^{\prime}} \rightarrow \text { ff } R W_{I_{\Gamma}^{\prime \prime}} \\
& \bigoplus_{b \in B} R W_{I_{\Gamma}^{\prime}}(?, \beta(b)) \mapsto \bigoplus_{b \in B \backslash B_{i}} R W_{I_{\Gamma}^{\prime \prime}}(? ?, \beta(b)) \\
& \left.h \mapsto \operatorname{pr}_{\bigoplus_{b \in B \backslash B_{i}}} R W_{I_{\Gamma}^{\prime \prime}(? ?, \beta(b))} \circ h\right|_{\bigoplus_{b \in B \backslash B_{i}} R W_{I_{\Gamma}^{\prime \prime}}(? ?, \beta(b))} \\
& F: \quad \quad \text { ff } R W_{I_{\Gamma}^{\prime \prime}} \rightarrow \text { ff } R W_{I_{\Gamma}^{\prime}} \\
& \bigoplus_{b \in B^{\prime \prime}} R W_{I_{\Gamma}^{\prime \prime}}(?, \beta(b)) \mapsto \bigoplus_{b \in B^{\prime \prime}} R W_{I_{\Gamma}^{\prime}}(? ?, \beta(b)) \\
& h \mapsto \begin{cases}h & \text { if } ? ? \in W_{I_{\Gamma}^{\prime \prime}} \\
0 & \text { if } ? ? \in W_{i}\end{cases}
\end{aligned}
$$


by defining them on the equivalent subcategory of finite free $R W_{I_{\Gamma}^{\prime} \text {-modules }}$ of this special form. We obtain a commutative diagram with exact rows

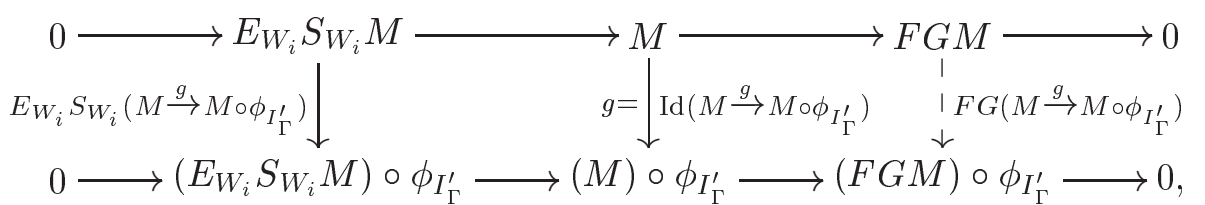

and we have an induced map on the cokernels which we denote by $F G(M \stackrel{g}{\rightarrow}$

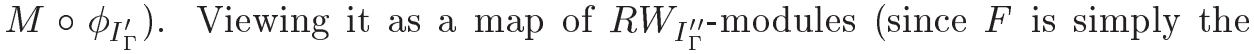
inclusion and $\phi_{I_{\Gamma}^{\prime}}$ can be restricted to $\left.W_{I_{\Gamma}^{\prime \prime}}\right)$ gives $G\left(M \stackrel{g}{\rightarrow} M \circ \phi_{I_{\Gamma}^{\prime}}\right): G M \rightarrow$ $(G M) \circ \phi_{I_{\Gamma}^{\prime \prime}}$ (One can also formulate this differently: $G F G(M)=G(M)$, so applying $G$ yields $G F G\left(M \stackrel{g}{\rightarrow} M \circ \phi_{I_{\Gamma}^{\prime}}\right)=: G\left(M \stackrel{g}{\rightarrow} M \circ \phi_{I_{\Gamma}^{\prime}}\right)$.) The functor $F$ on morphisms is simply the inclusion, i.e., viewing the morphisms as morphisms of $R W_{I_{\Gamma}^{\prime} \text {-modules. }}$

Given a morphism $h: M \rightarrow M^{\prime}$, where $M^{\prime}=\bigoplus_{b^{\prime} \in B^{\prime}} R W_{I_{\Gamma}^{\prime}}\left(\right.$ ?, $\left.\beta^{\prime}\left(b^{\prime}\right)\right)$, by Corollary 5.1.9 the left cube of the diagram

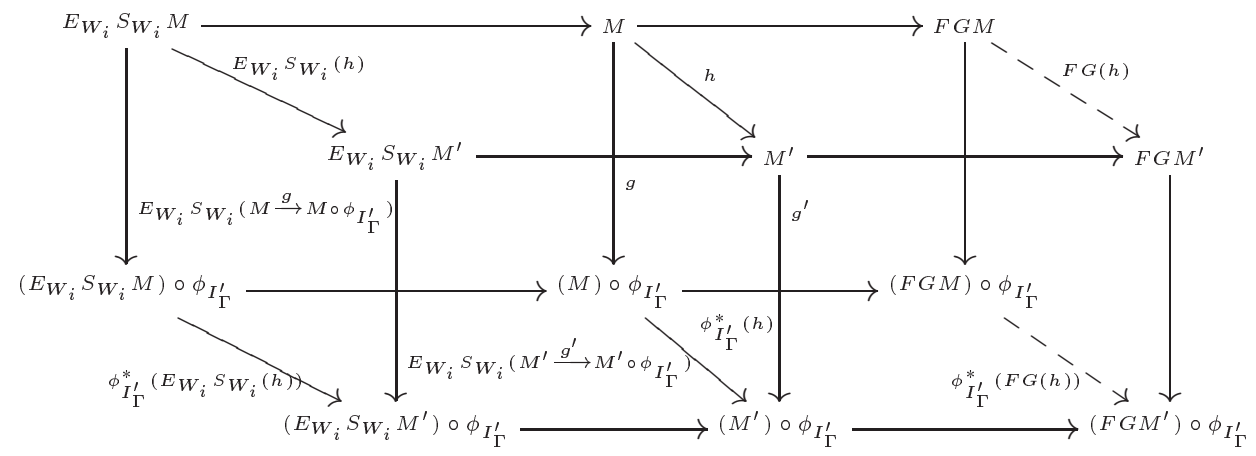

where the horizontal rows are short exact sequences also commutes. We obtain induced maps $F G(h)$ and $\phi_{I_{\Gamma}^{\prime}}^{*} F G(h)$ and a commutative diagram of the cokernels. The map $E_{W_{i}} S_{W_{i}}(h)$ is $\left.\operatorname{pr}_{\oplus_{b \in B_{i}}} R W_{I_{\Gamma}^{\prime}(?, \beta(b))} \circ h\right|_{\bigoplus_{b \in B_{i}} R W_{I_{\Gamma}^{\prime}}(?, \beta(b))}$. Again, one can obtain $G(h)$ by applying $G$ to $F G(h)$ and defining $G(h):=$ $G F G(h)$. Thus we have a short exact sequence of functors on $\phi_{I_{\Gamma}^{\prime}}-\operatorname{end}_{\mathrm{ff} R W_{I_{\Gamma}^{\prime}}}$

$$
0 \rightarrow E_{W_{i}} S_{W_{i}} \rightarrow \mathrm{Id} \rightarrow F G \rightarrow 0 .
$$

Since $E_{W_{i}} S_{W_{i}}$ is a functor, we have a natural transformation Id $\rightarrow E_{W_{i}} S_{W_{i}}$ given by $\left(M \stackrel{g}{\rightarrow} M \circ \phi_{I_{\Gamma}^{\prime}}\right) \mapsto E_{W_{i}} S_{W_{i}}\left(M \stackrel{g}{\rightarrow} M \circ \phi_{I_{\Gamma}^{\prime}}\right)$. This is a splitting map since the composition

$$
\begin{aligned}
E_{W_{i}} S_{W_{i}} & \rightarrow \mathrm{Id} \rightarrow E_{W_{i}} S_{W_{i}} \\
E_{W_{i}} S_{W_{i}}\left(M \stackrel{g}{\rightarrow} M \circ \phi_{I_{\Gamma}^{\prime}}\right) & \mapsto E_{W_{i}} S_{W_{i}}\left(M \stackrel{g}{\rightarrow} M \circ \phi_{I_{\Gamma}^{\prime}}\right) \\
& \mapsto E_{W_{i}} S_{W_{i}}\left(E_{W_{i}} S_{W_{i}}\left(M \stackrel{g}{\rightarrow} M \circ \phi_{I_{\Gamma}^{\prime}}\right)\right)
\end{aligned}
$$


is naturally equivalent to the identity: We have already shown that we have a natural equivalence $\tau: S_{W_{i}} E_{W_{i}} \rightarrow \mathrm{Id}$, so $E_{W_{i}} \tau_{S_{W_{i}}(-)}$ is a natural equivalence between the above composition and the identity on $E_{W_{i}} S_{W_{i}}$. So our sequence is split exact, i.e., a cofibration sequence.

By the Waldhausen additivity theorem, we obtain

$$
K\left(E_{W_{i}}\right) K\left(S_{W_{i}}\right)+K(F) K(G)=\mathrm{Id} .
$$

The following diagram commutes on the outside because of this equation and on the top because of the induction hypothesis $E_{\Gamma, \phi, I_{\Gamma}^{\prime \prime}} \circ S_{\Gamma, \phi, I_{\Gamma}^{\prime \prime}}=$ Id.

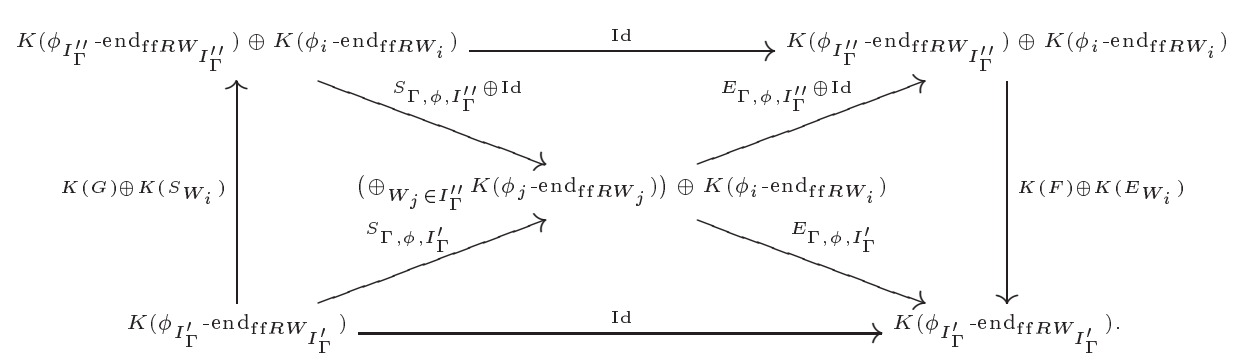

The right triangle commutes if $\left(K(F) \oplus K\left(E_{W_{i}}\right)\right) \circ\left(E_{\Gamma, \phi, I_{\Gamma}^{\prime \prime}} \oplus \mathrm{Id}\right)=E_{\Gamma, \phi, I_{\Gamma}^{\prime}}$, i.e., if

$$
K(F) \circ\left(\oplus_{W_{j} \in I_{\Gamma}^{\prime \prime}} K\left(E_{W_{j}}\right)\right) \oplus K\left(E_{W_{i}}\right)=\oplus_{W_{i} \in I_{\Gamma}^{\prime}} K\left(E_{W_{i}}\right) .
$$

This is obviously true: The $E_{W_{j}}$ on the left land in $\phi_{I_{\Gamma}^{\prime \prime}-e n d_{f f} R W_{I_{\Gamma}^{\prime \prime}}}$, and the functor $F$ is exactly what we need to push them forward to $\phi_{I_{\Gamma}^{\prime}}-\operatorname{end}_{\mathrm{ff}} R W_{I_{\Gamma}^{\prime}}$ where the $E_{W_{i}}$ on the right hand side land.

The left triangle commutes if $\left(S_{\Gamma, \phi, I_{\Gamma}^{\prime \prime}} \oplus \mathrm{Id}\right) \circ\left(K(G) \oplus K\left(S_{W_{i}}\right)\right)=S_{\Gamma, \phi, I_{\Gamma}^{\prime}}$, i.e., if

$$
\prod_{W_{j} \in I_{\Gamma}^{\prime \prime}} K\left(S_{W_{j}} \circ G\right) \oplus K\left(S_{W_{i}}\right)=\prod_{W_{i} \in I_{\Gamma}^{\prime}} K\left(S_{W_{i}}\right) .
$$

This is easily seen to be true, too: For $W_{j} \in I_{\Gamma}^{\prime \prime}$,

$$
S_{W_{j}} \circ G(M)=S_{W_{j}}\left(\bigoplus_{b \in B \backslash B_{i}} R W_{I_{\Gamma}^{\prime \prime}}(?, \beta(b))\right)=\bigoplus_{b \in B_{j}} R W_{j}(?, \beta(b))=S_{W_{j}}(M)
$$

and

$$
\begin{aligned}
& S_{W_{j}} \circ G\left(M \stackrel{g}{\rightarrow} M \circ \phi_{I_{\Gamma}^{\prime}}\right) \\
& =S_{W_{j}}\left(\left.\operatorname{pr}_{\oplus_{b \in B \backslash B_{i}} R W_{I_{\Gamma}^{\prime \prime}}\left(\phi_{I_{\Gamma}^{\prime \prime}}(?), \beta(b)\right)} \circ g\right|_{\bigoplus_{b \in B \backslash B_{i}} R W_{I_{\Gamma}^{\prime \prime}}(?, \beta(b))}\right) \\
& =\left.\operatorname{pr}_{\oplus_{b \in B_{j}}} R W_{j}\left(\phi_{j}(?), \beta(b)\right) \circ g\right|_{\oplus_{b \in B_{j}} R W_{j}(?, \beta(b))} \\
& =S_{W_{j}}\left(M \stackrel{g}{\rightarrow} M \circ \phi_{I_{\Gamma}^{\prime}}\right) \text {. }
\end{aligned}
$$


So the left triangle also commutes. We conclude that the bottom triangle commutes and thus that $E_{\Gamma, \phi, I_{\Gamma}^{\prime}} \circ S_{\Gamma, \phi, I_{\Gamma}^{\prime}}=\mathrm{Id}$. Therefore $E_{\Gamma, \phi}$ and $S_{\Gamma, \phi}$ are inverse isomorphisms.

It remains to show naturality. Given a covariant functor $F:\left(\Gamma_{1}, \phi\right) \rightarrow$ $\left(\Gamma_{2}, \psi\right)$, we have $S E \cong \mathrm{Id}$ and $E F \cong F E$, so $S F E \cong S E F \cong F$ and the following diagram commutes:

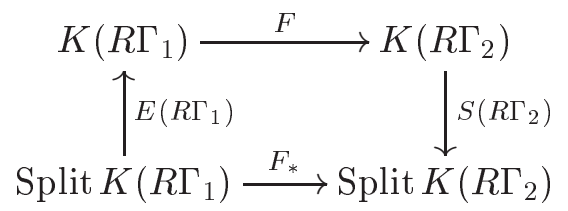

This also implies that $S F \cong S F E S \cong F S$, and so $S$ is a natural transformation.

From the proof we can deduce the following corollary.

Corollary 5.3.2. For any EI-category $\Gamma$ with endofunctor $\phi$, we have a natural splitting

$$
K\left(\operatorname{Id}_{(\Gamma, \phi)}\right)=\bigoplus_{W_{i} \in I_{\Gamma}} K\left(E_{W_{i}} S_{W_{i}}\right): K\left(\phi-\operatorname{end}_{\mathrm{ff} R \Gamma}\right) \rightarrow K\left(\phi-\operatorname{end}_{\mathrm{ff} R \Gamma}\right) .
$$

In particular, in $K_{0}\left(\phi-\operatorname{end}_{\mathrm{ff} R \Gamma}\right)$ we have for $(M \stackrel{g}{\rightarrow} M \circ \phi) \in \phi$-end $\mathrm{fff}_{\mathrm{ff}}$ the equality $[g]=\sum_{W_{i} \in I_{\Gamma}}\left[g_{i}\right]$, where $g_{i}:=E_{W_{i}} S_{W_{i}}(M \stackrel{g}{\rightarrow} M \circ \phi)$.

\section{$5.4 \quad$ A Finer Splitting Result}

Now we will split up the $K$-theory of each $\left(W_{i}, \phi_{i}\right)$ even further. The set of objects of $W_{i}$ can be partitioned into two subsets, the objects of $T_{i}$ and of $Q_{i}$. We establish an isomorphism

$$
K\left(\phi_{i}-\text { end }_{\mathrm{ff} R W_{i}}\right) \cong K\left(\phi_{T_{i}}-\mathrm{end}_{\mathrm{ff} R T_{i}}\right) \oplus K\left(\text { ff } R Q_{i}\right) .
$$

Let $W$ be a groupoid with endofunctor $\phi$. Since $W$ is a groupoid, every endofunctor is order-respecting.

Definition 5.4.1. Define $T$ to be the full subcategory of $W$ with objects

$$
\mathrm{Ob}(T):=\left\{x \in W \mid \text { there is } n \in \mathbb{N}_{>0} \text { with } \overline{\phi^{n}(x)}=\bar{x}\right\} .
$$

Define the length of $T$ to be

$$
l(T):=\min \left\{n \in \mathbb{N}_{>0} \mid \overline{\phi^{n}(x)}=\bar{x} \text { for } x \in T\right\} .
$$

For $y \in \mathrm{Ob}(W)$ define the height

$$
\operatorname{ht}(y):=\min \left\{n \in \mathbb{N} \mid \phi^{n}(y) \in \mathrm{Ob}(T)\right\} \in \mathbb{N} \cup\{\infty\} .
$$


Define the height of $W$ to be

$$
\operatorname{ht}(W):=\sup \{\operatorname{ht}(y) \mid y \in \mathrm{Ob}(W)\} .
$$

Define $Q_{j}$ for $j \geq 1$ to be the full subcategory of $W$ with

$$
\operatorname{Ob}\left(Q_{j}\right)=\{y \in \operatorname{Ob}(W) \mid \operatorname{ht}(y)=j\}
$$

and define $L_{j}$ for $j \geq 0$ to be the full subcategory of $W$ with

$$
\mathrm{Ob}\left(L_{j}\right)=\{y \in \mathrm{Ob}(W) \mid \operatorname{ht}(y) \leq j\},
$$

then $L_{j}=T \cup Q_{1} \cup \ldots \cup Q_{j}$. We set $Q:=\bigcup_{j=1}^{\text {ht }(W)} Q_{j}=W \backslash T$.

Note that the set of isomorphism classes of the groupoid $T$ is always finite, and nonempty if the set Is $W$ is finite. The set of isomorphism classes of the groupoid $Q$ can be finite or infinite.

We will split off the $Q_{j}$ one at a time, starting from the top. We treat groupoids $W$ with finite height. We can also treat groupoids that are a colimit of their finite height subgroupoids using a colimit argument. The only case which is then not covered is when $T=\emptyset$ and all objects in $W$ have height $\infty$.

We can restrict $\phi$ to $T$ and to each of the $L_{j}$ for $j \geq 0$. We call these restricted endofunctors $\phi_{T}: T \rightarrow T$ and $\phi_{L_{j}}: L_{j} \rightarrow L_{j}$. Note that $\phi\left(L_{j}\right) \subseteq$ $L_{j-1}$ for all $j \geq 1$. We define an abelian group

Finesplit $K\left(\phi-\operatorname{end}_{\mathrm{ff} R W}\right):=K\left(\phi_{T}-\operatorname{end}_{\mathrm{ffRT}}\right) \oplus K(\mathrm{ff} R Q)$.

To stick with the notation used before, this can also be seen as

Finesplit $K((W, \phi)):=K\left(\phi_{T}-\operatorname{end}_{\mathrm{ff} R T}\right) \oplus K(\mathrm{ff} R Q)$.

for a groupoid $W$ with endofunctor $\phi$. It is clear that we have the equation $K($ ff $R Q)=\bigoplus_{j=1}^{\mathrm{ht}(W)} K\left(\right.$ ff $\left.R Q_{j}\right)$. We define functors

$A: \phi_{T}-\operatorname{end}_{\mathrm{ff} R T} \oplus \mathrm{ff} R Q \rightarrow \phi-\operatorname{end}_{\mathrm{ff} R W}$

$$
\begin{aligned}
& \left(P \stackrel{l}{\rightarrow} P \circ \phi_{T}, N\right) \mapsto\left(\operatorname{ind}_{T \subseteq W} P \oplus \operatorname{ind}_{Q \subseteq W} N \frac{\left({ }^{\left(\operatorname{incl}_{T} \subseteq W\right)_{*}(l)} \begin{array}{l}
0 \\
0
\end{array}\right)}{\longrightarrow}\right. \\
& \left.\left(\operatorname{ind}_{T \subseteq W} P \oplus \operatorname{ind}_{Q \subseteq W} N\right) \circ \phi\right) \\
& \left(h_{1}, h_{2}\right) \mapsto\left(\operatorname{ind}_{T \subseteq W} h_{1} \oplus \operatorname{ind}_{Q \subseteq W} h_{2}\right)
\end{aligned}
$$

and

$$
\begin{aligned}
B: \quad \phi-\operatorname{end}_{\mathrm{ff} R W} & \rightarrow \phi_{T}-\operatorname{end}_{\mathrm{ff} R T} \oplus \mathrm{ff} R Q \\
\left(M \stackrel{g}{\rightarrow} M \circ \phi_{T}\right) & \mapsto\left(\operatorname{res}_{T \subseteq W} M \stackrel{\operatorname{res}_{T \subseteq W}(g)}{\longrightarrow}\left(\operatorname{res}_{T \subseteq W} M\right) \circ \phi_{T}\right. \\
& \left.\operatorname{res}_{Q \subseteq W} M\right) \\
h & \mapsto\left(\operatorname{res}_{T \subseteq W} h, \operatorname{res}_{Q \subseteq W} h\right)
\end{aligned}
$$


where $\operatorname{res}_{T \subseteq W}(g)$ is defined to be the composition

$$
\operatorname{res}_{T \subseteq W}(g): \operatorname{res}_{T \subseteq W} M \stackrel{\operatorname{res}_{T \subseteq W}(g)}{\longrightarrow} \operatorname{res}_{T \subseteq W}(M \circ \phi) \stackrel{\sim}{\rightarrow}\left(\operatorname{res}_{T \subseteq W} M\right) \circ \phi_{T} .
$$

This map is known from Section 2.2. Here we use the notation $\operatorname{res}_{T \subseteq W}:=$ $\operatorname{res}_{i_{n c l} l_{T W}}$ and $\operatorname{ind}_{T \subseteq W}:=$ ind $_{\text {incl }_{T \subseteq W}}$. Note that for groupoids res $x=S_{x}$ since we do not have any morphisms that are not isomorphisms. So in this case res $_{x}$ preserves "finitely generated free" since $S_{x}$ does. These functors induce maps

$$
K(A): \quad \text { Finesplit } K\left(\phi-\operatorname{end}_{\mathrm{ff} R W}\right) \rightarrow K\left(\phi-\operatorname{end}_{\mathrm{ff} R W}\right)
$$

and $K(B): \quad K\left(\phi-\right.$ end $\left._{\mathrm{ffRW}}\right) \rightarrow$ Finesplit $K\left(\phi-\right.$ end $\left._{\mathrm{ff} R W}\right)$.

We will show that these are isomorphisms inverse to one another if $W$ is a groupoid that is a colimit of its finite height subgroupoids $L_{j}$ for $j<\infty$.

Theorem 5.4.2. Let $W$ be a groupoid with endofunctor $\phi$ that is a colimit of its finite height subgroupoids. Then we have an isomorphism of abelian groups

$$
K\left(\phi-\operatorname{end}_{\mathrm{ff} R W}\right) \cong K\left(\phi_{T}-\operatorname{end}_{\mathrm{ff} R T}\right) \oplus K(\mathrm{ff} R Q)
$$

which is given by $K(B)$ with inverse $K(A)$.

Proof. 1) $K(B \circ A)=\operatorname{Id}_{K\left(\phi_{T}-e^{-e n d} f_{f f R T}\right) \oplus K(f f R Q)}$

We show that $B \circ A$ is naturally equivalent to $\operatorname{Id}_{\phi_{T} \text {-end ffRT } \oplus \text { ff } R Q \text {. Let }}$ $\left(P \stackrel{l}{\rightarrow} P \circ \phi_{T}, N\right) \in \phi_{T}-\operatorname{end}_{\mathrm{ff} R T} \oplus \mathrm{ff} R Q$. Then

$$
\begin{aligned}
& B \circ A\left(P \stackrel{l}{\rightarrow} P \circ \phi_{T}, N\right) \\
& =\left(\operatorname{res}_{T \subseteq W}\left(\operatorname{ind}_{T \subseteq W} P \oplus \operatorname{ind}_{Q \subseteq W} N\right) \stackrel{\operatorname{res}_{T \subseteq W}\left(\begin{array}{ll}
\left(\operatorname{incl}_{T}{ }_{0} W\right)_{*}(l) & 0 \\
0
\end{array}\right)}{\longrightarrow}\right. \\
& \left(\operatorname{res}_{T \subseteq W}\left(\operatorname{ind}_{T \subseteq W} P \oplus \operatorname{ind}_{Q \subseteq W} N\right)\right) \circ \phi_{T}, \\
& \left.\operatorname{res}_{Q \subseteq W}\left(\operatorname{ind}_{T \subseteq W} P \oplus \operatorname{ind}_{Q \subseteq W} N\right)\right) \\
& =\left(\operatorname{res}_{T \subseteq W} \operatorname{ind}_{T \subseteq W} P \stackrel{\operatorname{res}_{T \subseteq W}\left(\operatorname{incl}_{T \subseteq W}\right)_{*}(l)}{\longrightarrow}\left(\operatorname{res}_{T \subseteq W} \operatorname{ind}_{T \subseteq W} P\right) \circ \phi_{T},\right. \\
& \left.\operatorname{res}_{Q \subseteq W} \operatorname{ind}_{Q \subseteq W} N\right) \\
& \cong\left(P \stackrel{l}{\rightarrow} p \circ \phi_{T}, N\right) \text {. }
\end{aligned}
$$

Here $\operatorname{res}_{Q \subseteq W}\left(\operatorname{ind}_{T \subseteq W} P\right)=0$ and $\operatorname{res}_{T \subseteq W}\left(\operatorname{ind}_{Q \subseteq W} N\right)=0$ since $T$ and $Q$ are disjoint. Remember that $W$ is a groupoid, so all morphisms are isomorphisms, and $\operatorname{res}_{x}=S_{x}$. The bottom isomorphism is given by the natural transformations $\eta_{T \subseteq W}: P \rightarrow \operatorname{res}_{T \subseteq W}$ ind $_{T \subseteq W} P$ and $\eta_{Q \subseteq W}: N \rightarrow$ $\operatorname{res}_{Q \subseteq W} \operatorname{ind}_{Q \subseteq W} N$ which are isomorphisms since $T \subseteq W$ and $Q \subseteq W$ are inclusions of full subcategories. (This is analogous to Section 2.2, too.) 
Let $\left(h_{1}, h_{2}\right)$ be a morphism, then

$$
\begin{aligned}
B & \circ A\left(h_{1}, h_{2}\right) \\
& =\left(\operatorname{res}_{T \subseteq W}\left(\operatorname{ind}_{T \subseteq W} h_{1} \oplus \operatorname{ind}_{Q \subseteq W} h_{2}\right), \operatorname{res}_{Q \subseteq W}\left(\operatorname{ind}_{T \subseteq W} h_{1} \oplus \operatorname{ind}_{Q \subseteq W} h_{2}\right)\right) \\
& =\left(\operatorname{res}_{T \subseteq W} \operatorname{ind}_{T \subseteq W} h_{1}, \operatorname{res}_{Q \subseteq W} \operatorname{ind}_{Q \subseteq W} h_{2}\right) \\
& \cong\left(h_{1}, h_{2}\right) .
\end{aligned}
$$

2) $\left.K(A \circ B)=\operatorname{Id}_{K(\phi-e n d} d_{\text {ff } R W}\right)$

We have $W=\operatorname{colim}_{k} L_{k}$ and $\phi-\operatorname{end}_{\mathrm{ff} R W}=\operatorname{colim}_{k}\left(\phi_{L_{j}}-\operatorname{end}_{\mathrm{ffR} R L_{j}}\right)$ by construction. We have $A \circ B=\left(\operatorname{colim}_{k} A_{k}\right) \circ\left(\operatorname{colim}_{k} B_{k}\right)=\operatorname{colim}_{k}\left(A_{k} \circ B_{k}\right)$, where $A_{k}$ and $B_{k}$ are the restrictions of $A$ and $B$ to $L_{k}$. Hence it suffices to show that for any finite $k$ we have $K\left(A_{k} \circ B_{k}\right)=\operatorname{Id}_{K\left(\phi_{L_{k}} \text {-end } d_{f f R L_{k}}\right)}$.

We proceed by induction over $k$. If $k=0$, then there is nothing to prove. Assume that $\mathrm{ht}(W)=k$ and that we have $K\left(A_{k-1} \circ B_{k-1}\right)=$ $\operatorname{Id}_{K\left(\phi_{L_{k-1}}-\text { end }_{\mathrm{ffRL}} L_{k-1}\right)}$. We have a short exact sequence

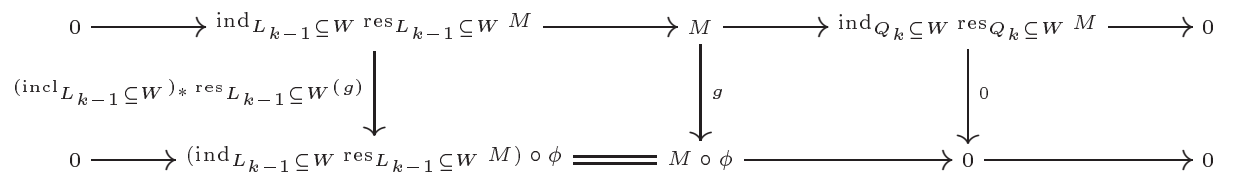

It is split exact since

$$
\left(\operatorname{incl}_{L_{k-1} \subseteq W}\right)_{*} \circ \operatorname{res}_{L_{k-1} \subseteq W}: \operatorname{Id} \rightarrow\left(\operatorname{incl}_{L_{k-1} \subseteq W}\right)_{*} \circ \operatorname{res}_{L_{k-1} \subseteq W}
$$

is a functorial splitting. Let iom: ff $R W \rightarrow \phi-\operatorname{end}_{\mathrm{ff} R W}$ denote the functor inserting the 0-map. By the Waldhausen additivity theorem we obtain

$$
\begin{aligned}
& K\left(\left(\operatorname{incl}_{L_{k-1} \subseteq W}\right)_{*}\right) K\left(\operatorname{res}_{L_{k-1} \subseteq W}\right)+K\left(\left.\operatorname{iom}_{0} \operatorname{ind}_{Q_{k} \subseteq W}\right|_{\text {Mod }}\right) K\left(\left.\operatorname{res}_{Q_{k} \subseteq W}\right|_{\text {Mod }}\right) \\
& =\operatorname{Id}_{K\left(\phi-\operatorname{end}_{\mathrm{ff} R W}\right)} .
\end{aligned}
$$

Thus the diagram

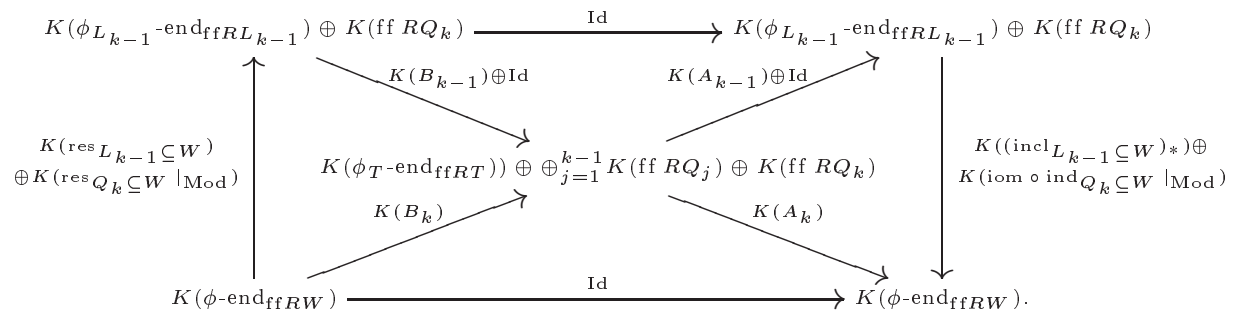

commutes on the outside. It is clear that $\left.\operatorname{res}_{Q_{j} \subseteq L_{k-1}}\right|_{\text {Mod }} \circ \operatorname{res}_{L_{k-1} \subseteq W}=$ $\left.\operatorname{res}_{Q_{j} \subseteq W}\right|_{\text {Mod }}$ and that $\operatorname{res}_{T \subseteq W}=\operatorname{res}_{T \subseteq L_{k-1}} \circ \operatorname{res}_{L_{k-1} \subseteq W}$, so the left triangle commutes. We also know that $\left(\operatorname{incl}_{L_{k-1} \subseteq W}\right)_{*} \circ\left(\operatorname{incl}_{T \subseteq L_{k-1}}\right)_{*}=\left(\operatorname{incl}_{T \subseteq W}\right)_{*}$ and that iom $\left.\circ \operatorname{ind}_{Q_{k} \subseteq W}\right|_{\text {Mod }}=\left.A_{k}\right|_{\text {ff } R Q_{k}}$, so the right triangle commutes. The top triangle commutes by the induction hypothesis, so the bottom triangle also commutes, and we are finished. 
Remark 5.4.3. We think it probable that this splitting also works in the case we have not treated yet, namely that $T=\emptyset$ and $W=Q$. If all elements in $W$ have height infinity, we can index the connected subgroupoids of $W$ by $j \in \mathbb{Z}$ such that $\left.\phi\right|_{Q_{j}}: Q_{j} \rightarrow Q_{j-1}$. We know that $\bigoplus_{j \in \mathbb{Z}} K\left(\right.$ ff $\left.R Q_{j}\right)=$ $\operatorname{colim}_{N \rightarrow-\infty} \bigoplus_{j>N} K\left(\mathrm{ff} R Q_{j}\right.$ ). Using the argument from the proof of Theorem 5.4.2, we have for any $N \in \mathbb{Z}$ an isomorphism

$$
K\left(\phi-\operatorname{end}_{\mathrm{ff} R \Gamma}\right) \cong K\left(\phi_{\Gamma_{N}}-\operatorname{end}_{\mathrm{ff} R \Gamma_{N}}\right) \oplus \bigoplus_{j>N} K\left(\text { ff } R Q_{j}\right),
$$

where $\Gamma_{N}$ is the full subgroupoid of $W$ with objects in $Q_{k}$ for any $k \leq$ $N$. The inclusion maps of the direct system commute with the inclusions $\bigoplus_{j>N} K\left(\mathrm{ff} R Q_{j}\right) \rightarrow K\left(\phi-\mathrm{end}_{\mathrm{ff} R \Gamma}\right)$, so we have an induced inclusion $\bigoplus_{j \in \mathbb{Z}} K\left(\mathrm{ff} R Q_{j}\right) \rightarrow K\left(\phi-\mathrm{end}_{\mathrm{ff} R \Gamma}\right)$. We need to show that it is surjective.

In $K_{0}$, the argument goes as follows: We work with endomorphisms of finite free modules, so for any module $M$ there is an $N_{M} \in \mathbb{Z}$ such that $M \cong \oplus_{i \in I} R \Gamma\left(?, m_{i}\right)$ with $m_{i} \in Q_{j_{i}}$ such that all $j_{i}>N_{M}$. Any element in $K_{0}\left(\phi\right.$-end $\left._{\mathrm{ffR} R}\right)$ can be represented by some $[g: M \rightarrow M \circ \phi]$, and this maps to $0+[M] \in K_{0}\left(\phi_{\Gamma_{N_{M}}}-\operatorname{end}_{\mathrm{ff} R \Gamma_{N_{M}}}\right) \oplus \bigoplus_{j>N_{M}} K_{0}\left(\right.$ ff $\left.R Q_{j}\right)$ under the above isomorphism, and thus it lies in the image of the map $\bigoplus_{j \in \mathbb{Z}} K_{0}\left(\mathrm{ff} R Q_{j}\right) \rightarrow K_{0}\left(\phi\right.$-end $\left._{\mathrm{ff} R \Gamma}\right)$.

Combining Theorem 5.4.2 with Theorem 5.3.1, we obtain the following corollary.

Corollary 5.4.4. Let $(\Gamma, \phi)$ be an EI-category $\Gamma$ with order-respecting endofunctor $\phi$ such that every $W_{i} \in I_{\Gamma}$ is the colimit of its finite height subgroupoids. Then

$$
K\left(\phi-\operatorname{end}_{\mathrm{ff} R \Gamma}\right) \cong \bigoplus_{W_{i} \in I_{\Gamma}}\left(K\left(\phi_{T_{i}}-\operatorname{end}_{\mathrm{ff} R T_{i}}\right) \oplus K\left(\mathrm{ff} R Q_{i}\right)\right) .
$$

The isomorphism is given by $\bigoplus_{W_{i} \in I_{\Gamma}}\left(K\left(S_{T_{i}}\right) \oplus K\left(\left.S_{Q_{i}}\right|_{\text {Mod }}\right)\right)$ with inverse $\bigoplus_{W_{i} \in I_{\Gamma}}\left(K\left(E_{T_{i}}\right) \oplus K\left(E_{Q_{i}}\right.\right.$ iom $\left.)\right)$.

Proof. From theorems 5.3.1 and 5.4.2 we know that the isomorphism is given by $\left(\oplus_{W_{i} \in I_{\Gamma}} K\left(B_{i}\right)\right) \circ S_{\Gamma, \phi}$ with inverse $E_{\Gamma, \phi} \circ \oplus_{W_{i} \in I_{\Gamma}} K\left(A_{i}\right)$. From the definitions of $S_{\Gamma, \phi}, E_{\Gamma, \phi}, A$ and $B$ in Sections 5.2 and 5.4 we deduce the above form. We use the fact that $\operatorname{res}_{T_{i}} S_{W_{i}}=S_{T_{i}}$ since $\operatorname{res}_{T_{i}} \operatorname{res}_{W_{i}}=\operatorname{res}_{T_{i}}$ and $W_{i}$ is a groupoid, $T_{i}$ subgroupoid, so anything that is divided out has to come from outside $W_{i}$, and that analogously $\operatorname{res}_{Q_{i}} S_{W_{i}}=S_{Q_{i}}$.

What about functoriality in $(W, \phi) \in$ Endo(EI-Cat) ${ }_{\text {ord }}$ ? Not all our constructions are compatible with a functor $F:(W, \phi) \rightarrow\left(W^{\prime}, \phi^{\prime}\right)$ of groupoids with endofunctor, i.e., $F: W \rightarrow W^{\prime}$ such that $F \circ \phi=\phi^{\prime} \circ F$. The inclusion of $\left(T, \phi_{T}\right)$ into $(W, \phi)$ is compatible with $F$, but the restriction from $(W, \phi)$ 
to $\left(T, \phi_{T}\right)$ is not: Modules lying over $Q$ would be forgotten by $\operatorname{res}_{T \subseteq W}$, but they might be mapped to $T^{\prime}$ under $F$, so their images would be seen by $\operatorname{res}_{T^{\prime} \subseteq W^{\prime}}$. So Finesplit $K$ is not a functor on the category of groupoids with endofunctors.

Remark 5.4.5. These splitting results can be of independent interest in algebraic $K$-theory. One can apply them to endomorphism categories of modules over complicated rings and split up the $K$-theory of these endomorphism categories into simpler parts. This could have interesting applications.

\subsection{A Small Example}

In this section, we will briefly discuss an example which should help to enlighten the statement of the splitting result. Let $\Gamma$ be the category with two objects $a$ and $b$, with $\operatorname{Aut}(a)=H$ and $\operatorname{Aut}(b)=G$, and with no morphism between $a$ and $b$.
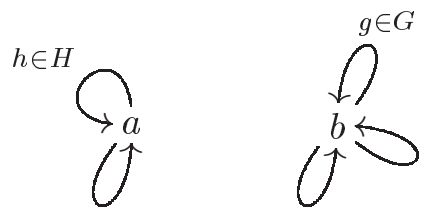

An $R \Gamma$-module consists of a pair, an $R H$-module and an $R G$-module. Let $\phi: \Gamma \rightarrow \Gamma$ be the functor given by $a \mapsto b, b \mapsto b,\left.\phi\right|_{H}: H \rightarrow G$ any group homomorphism and $\left.\phi\right|_{G}=\mathrm{id}_{G}$. Then, in the notation of this chapter, $W=\{a, b\}, T=\{b\}$ and $Q=\{a\}$. The splitting result yields

$$
\begin{aligned}
K_{0}\left(\phi-\operatorname{end}_{\mathrm{ff} R \Gamma}\right) & =K_{0}\left(\mathrm{id}_{-}-\operatorname{end}_{\mathrm{ff} R T}\right) \oplus K_{0}(\text { ff } R Q) \\
& =K_{0}(\operatorname{End}(\text { ff } R G)) \oplus K_{0}(\text { ff } R H) .
\end{aligned}
$$




\section{Chapter 6}

\section{The Geometric Meaning of the Splitting Results}

In this chapter, we describe the geometric meaning of the splitting results obtained in Chapter 5, in particular of Corollary 5.4.4. Here the fixed point sets corresponding to the different subgroups $H \leq G$ come into play. We also show how to get back from groupoids to groups, thereby obtaining a description of the universal functorial equivariant Lefschetz invariant $\left(U_{G}^{\mathbb{Z}}(X, f), u_{G}^{\mathbb{Z}}(X, f)\right)$ in terms of the fundamental groups of the fixed point sets instead of their fundamental groupoids.

The result of Corollary 5.4.4 gives an expression of the universal functorial equivariant Lefschetz invariant $u_{G}^{\mathbb{Z}}(X, f)=\left[C^{c}(\widetilde{f})\right] \in K_{0}(\phi$-end $\operatorname{ff\mathbb {Z}} \Pi(G, X))$ in terms of the maps $C^{c}\left(\left.\widetilde{f}\right|_{\widetilde{X}^{H}(x)},\left.\widetilde{f}\right|_{\widetilde{X}^{>H}(x)}\right)$ on the relative cellular chain complexes $C^{c}\left(\widetilde{X}^{H}(x), \widetilde{X}^{>H}(x)\right)$, for subgroups $H \leq G$ and objects $x \in$ $\Pi(G, X)$ that correspond to points with isotropy group $H$.

\subsection{Reduction to Groups instead of Groupoids}

In this section, we will show how to replace the groupoids by groups [Lüc89, Remark 10.35]. This is rather technical, so it is helpful to have the geometric picture in mind.

Given a topological space $X$ with endomorphism $f: X \rightarrow X$, one usually prefers working with the fundamental group (after the choice of a basepoint, which does not play a role if $X$ is connected) instead of the fundamental groupoid. The transition from the fundamental groupoid to the fundamental group is done as follows: Assuming $X$ connected, we choose a basepoint $x \in X$ and look at the fundamental group with respect to this basepoint. In order for the endomorphism $f: X \rightarrow X$ to induce an endomorphism $\phi: \pi_{1}(X, x) \rightarrow \pi_{1}(X, x)$, we also need to choose a path $v$ from $x$ to $f(x)$. We define $\phi:=c_{v} \circ \pi_{1}(f)$, where $c_{v}$ is the conjugation 
map $c_{v}: \pi_{1}(X, f(x)) \rightarrow \pi_{1}(X, x), \gamma \mapsto v \gamma v^{-1}$. Here composition is written from left to right, as is usual for composition of paths, so $v \gamma v^{-1}$ stands for $\left[v * \gamma * v^{-1}\right]$. Note that choosing a path $v$ from $x$ to $f(x)$ corresponds to choosing a morphism $w=(\sigma,[v]) \in \operatorname{Mor}(f(x), x)$.

Algebraically, we are interested in the categories $\phi_{T_{i}}$-end $\mathrm{ff}_{R T_{i}}$ and ff $R Q_{i}$. We start with the easier task, namely the study of ff $R Q_{i}$. The category $Q_{i}$ is a disjoint union of connected groupoids $Q_{i j}$. A finitely generated free $R Q_{i}$-module is a collection of finitely generated free $R Q_{i j}$-modules. We can choose an object $x_{i j}$ in every component $Q_{i j}$. We know that the inclusion $x_{i j} \subseteq \overline{x_{i j}}$ of an object into its isomorphism class induces an equivalence of categories $\widehat{\operatorname{Aut}\left(x_{i j}\right)} \rightarrow \Gamma\left[x_{i j}\right]=Q_{i j}$, where $\widehat{\operatorname{Aut}\left(x_{i j}\right)}$ is the group $\operatorname{Aut}\left(x_{i j}\right)$ viewed as a category (with one object). So by restricting with this equivalence of categories we can view a finitely generated free $R Q_{i j}$-module as a finitely generated free $R \widehat{\operatorname{Aut}\left(x_{i j}\right)}$-module. An $R \widehat{\operatorname{Aut}\left(x_{i j}\right)}$-module is canonically an $R \operatorname{Aut}\left(x_{i j}\right)$-module and vice versa [Lüc89], so a finitely generated free $R Q_{i j}$-module can be seen as a finitely generated free $R \operatorname{Aut}\left(x_{i j}\right)$-module.

In a precise technical formulation, we have a restriction functor

$$
\begin{aligned}
\operatorname{res}_{\amalg \text { incl }_{x_{i j}}} \text { : ff } R Q_{i} & \rightarrow \coprod_{j \in J_{i}} \text { ff } R \operatorname{Aut}\left(x_{i j}\right) \\
M & \mapsto \coprod_{j \in J_{i}} \operatorname{res}_{\text {incl }_{x_{i j}}} M \\
h & \mapsto \coprod_{j \in J_{i}} \operatorname{res}_{\text {incl }_{x_{i j}}} h .
\end{aligned}
$$

We also have an induction functor

$$
\begin{aligned}
\operatorname{ind}_{\amalg \operatorname{incl}_{x_{i j}}: \coprod_{j \in J_{i}} \operatorname{ff} R \operatorname{Aut}\left(x_{i j}\right)} & \rightarrow \text { ff } R Q_{i} \\
\coprod_{j \in J_{i}} N_{j} & \mapsto \bigoplus_{j \in J_{i}} \operatorname{ind}_{\text {incl }_{x_{i j}}} N_{j}, \\
\coprod_{j \in J_{i}} h_{j} & \mapsto \bigoplus_{j \in J_{i}} \text { ind }_{\text {incl }_{x_{i j}}} h_{j} .
\end{aligned}
$$

These are equivalences of categories inverse to each other, as stated in Section 2.2. The natural transformations $\eta$ and $\varepsilon$ are in this case natural equivalences because $\widehat{\operatorname{Aut}\left(x_{i j}\right)} \rightarrow Q_{i j}$ is an equivalence of categories. So the group homomorphism

$$
K\left(\operatorname{res}_{\amalg \operatorname{incl}_{x_{i j}}}\right): K\left(\operatorname{ff} R Q_{i}\right) \rightarrow K\left(\coprod_{j \in J_{i}} \operatorname{ff} R \operatorname{Aut}\left(x_{i j}\right)\right)=\bigoplus_{j \in J_{i}} K\left(\operatorname{ff} R \operatorname{Aut}\left(x_{i j}\right)\right)
$$

is an isomorphism, and we might feel more comfortable looking at the universal functorial equivariant Lefschetz invariant in $\bigoplus_{j \in J_{i}} K_{0}\left(\operatorname{ff} R \operatorname{Aut}\left(x_{i j}\right)\right)$ 
if we prefer working with groups instead of groupoids. The choice of the objects $x_{i j} \in Q_{i j}$ does not play a role, we can use the isomorphisms

$$
K\left(\operatorname{res}_{\amalg \operatorname{incl}_{x_{i j}^{\prime}}} \operatorname{ind}_{\amalg \operatorname{incl}_{x_{i j}}}\right): \bigoplus_{j \in J_{i}} K\left(\operatorname{ff} R \operatorname{Aut}\left(x_{i j}\right)\right) \stackrel{\sim}{\rightarrow} \bigoplus_{j \in J_{i}} K\left(\operatorname{ff} R \operatorname{Aut}\left(x_{i j}^{\prime}\right)\right)
$$

to identify the groups obtained from different choices. We define

$$
V_{Q_{i j}}:=\coprod_{x_{i j} \in Q_{i j}} K\left(\operatorname{ff} R \operatorname{Aut}\left(x_{i j}\right)\right) / \sim
$$

and

$$
V_{Q_{i}}:=\bigoplus_{j \in J_{i}} V_{Q_{i j}}
$$

where for $a \in K\left(\right.$ ff $\left.R \operatorname{Aut}\left(x_{i j}\right)\right)$ and $a^{\prime} \in K\left(R \operatorname{Aut}\left(x_{i j}^{\prime}\right)\right)$ we set

$$
a \sim a^{\prime} \Leftrightarrow K\left(\operatorname{res}_{\amalg \operatorname{incl}_{x_{i j}^{\prime}}} \operatorname{ind}_{\amalg \operatorname{incl}_{x_{i j}}}\right)(a)=a^{\prime} .
$$

The abelian groups $V_{Q_{i j}}$ and $V_{Q_{i}}$ are well-defined: Existence of the natural equivalence $\eta$ implies that $\left.K\left(\operatorname{res}_{\amalg \operatorname{incl}_{x}} \operatorname{ind}_{\amalg \operatorname{incl}_{x_{i j}}}\right)=\operatorname{Id}_{K(f f} R \operatorname{Aut}\left(x_{i j}\right)\right)$, and $K\left(\operatorname{res}_{\amalg \text { incl }_{x_{i j}^{\prime \prime}}} \operatorname{ind}_{\amalg \operatorname{incl}_{x^{\prime} j}} \operatorname{res}_{\amalg \text { incl }_{x_{i j}^{\prime}}} \operatorname{ind}_{\amalg \operatorname{incl}_{x_{i j}}}\right)=K\left(\operatorname{res}_{\amalg \operatorname{incl}_{x_{i j}^{\prime \prime}}} \operatorname{ind}_{\amalg \operatorname{incl}_{x_{i j}}}\right)$ is true because we have $\left.K\left(\operatorname{ind}_{\amalg \text { incl }_{x_{i j}^{\prime}}} \operatorname{res}_{\amalg \text { incl }_{x_{i j}^{\prime}}}\right)=\operatorname{Id}_{K(\mathrm{ff}} R Q_{i}\right)$ by the existence of the natural equivalence $\varepsilon$. We have obtained a "basepoint free" description in terms of groups instead of groupoids.

For any choice of elements $x_{i j}$, the map $\operatorname{res}_{\amalg \text { incl }} x_{i j}$ induces the same isomorphism

$$
\xi_{Q_{i}}: K\left(\mathrm{ff} R Q_{i}\right) \stackrel{\sim}{\rightarrow} V_{Q_{i}} .
$$

The case of the category $\phi_{T_{i}}-\operatorname{end}_{\mathrm{ff} R T_{i}}$ is more difficult since there is the endofunctor $\phi_{T_{i}}$ involved. We write $T$ instead of $T_{i}$ for simplicity of notation. Note that $T$ is a disjoint union of connected groupoids. Choosing an object $y \in T$, we can write $T$ as $T=\bar{y} \amalg \overline{\phi(y)} \amalg \cdots \amalg \overline{\phi^{l-1}(y)}$, with $\overline{\phi^{l}(y)}=\bar{y}$, where as usual we identify $\bar{y}$ with the groupoid it generates by taking the full subcategory of $\Gamma$ generated by the objects of $\bar{y}$, namely $\bar{y}=\Gamma[y]$. As above, we want to replace these groupoids $\overline{\phi^{i}(y)}$ by the groups $\operatorname{Aut}\left(\phi^{i}(y)\right)$.

Having chosen $y \in \bar{y}$, we have determined objects $\phi(y), \ldots, \phi^{l-1}(y)$ in the other connected components of $T$. We also need to choose a morphism $w$ from $\phi^{l}(y)$ to $y$. We can find such a $w$ since $\overline{\phi^{l}(y)}=\bar{y}$. The morphism $w$ is invertible since $\Gamma[y]$ is a groupoid.

As above, we know that the inclusion $\phi^{i}(y) \subseteq \overline{\phi^{i}(y)}$ induces an equivalence of categories Aut( $\left.\widehat{\phi^{i}(y)}\right) \rightarrow \Gamma\left[\phi^{i}(y)\right]$. Again we can take the groups $\operatorname{Aut}(y), \ldots, \operatorname{Aut}\left(\phi^{l-1}(y)\right)$ and group homomorphisms between them instead of the categories $\left.\widehat{\operatorname{Aut}(y)}, \ldots, \operatorname{Aut} \widehat{\left(\phi^{l-1}\right.}(y)\right)$ and natural transformations between them since for any group $G$ an $R G$-module is canonically an $R \widehat{G}$ module and vice versa [Lüc89]. 
For $\gamma \in \operatorname{Aut}\left(\phi^{l}(y)\right)$ we set $c_{w}(\gamma):=w \gamma w^{-1} \in \operatorname{Aut}(y)$. We obtain a group homomorphism $\phi_{y, w}: \operatorname{Aut}\left(\phi^{l-1}(y)\right) \stackrel{\left.\phi\right|_{\operatorname{Aut}\left(\phi^{l-1}(y)\right)}}{\longrightarrow} \operatorname{Aut}\left(\phi^{l}(y)\right) \stackrel{c_{w}}{\longrightarrow} \operatorname{Aut}(y)$.

On the groupoid side, we can view $\phi$-end $\mathrm{ff}_{\mathrm{ff}}$ as being made up of modules over the "circle" of groupoids and functors

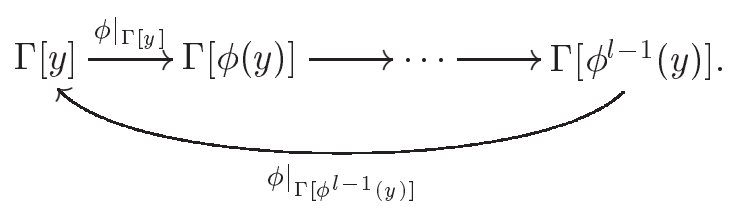

This is the notation which is well adapted to viewing the groups. When we use this notation, we call the category of modules over this "circle" category $\mathrm{ff} R \Gamma[y]$-Circles. This is the same as $\phi$-end $\mathrm{eff}_{R T}$.

We also have a "circle" of groups and group homomorphisms

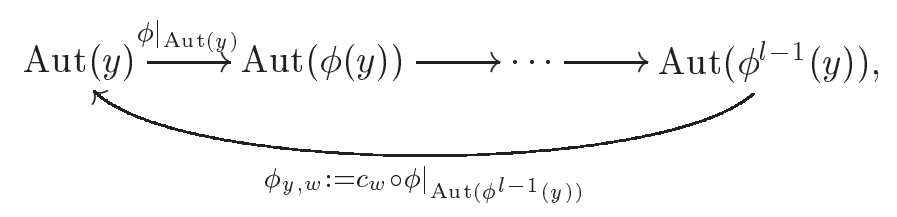

In analogy to the groupoid case, we define a module over this to be a "circle" of modules and morphisms

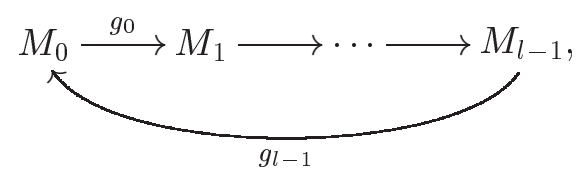

where $M_{i}$ is an $R \operatorname{Aut}\left(\phi^{i}(y)\right)$-module and for $0 \leq i<l-1$ the map $g_{i}$ is a $\left.\phi\right|_{\text {Aut }\left(\phi^{i}(y)\right)}$-morphism, i.e., we have $g_{i}:\left.M_{i} \rightarrow M_{i+1} \circ \phi\right|_{\operatorname{Aut}\left(\phi^{i}(y)\right)}$. For $i=l-1$ the map $g_{l-1}$ is a $\phi_{y, w}$-morphism, i.e., we have $g_{l-1}: M_{l-1} \rightarrow M_{0} \circ \phi_{y, w}$. A finite free module is defined to be one where all modules $M_{0}, \ldots, M_{l-1}$ are finite and free. A morphism $h:\left(M_{i}\right)_{0 \leq i \leq l-1} \rightarrow\left(M_{i}^{\prime}\right)_{0 \leq i \leq l-1}$ between these modules is a set $\left\{h_{0}, \ldots, h_{l-1}\right\}$ of maps $h_{i}: M_{i} \rightarrow \bar{M}_{i}^{\prime}$ such that all resulting diagrams commute. We call the category of these modules ffRAut(y)-Circles.

We want to prove the following theorem.

Theorem 6.1.1. Let $\Gamma$ be an EI-category, and let $y \in \mathrm{Ob}(\Gamma)$ be an object of $\Gamma$. There is an equivalence of categories

$$
\mathrm{ff} R \Gamma[y] \text {-Circles } \stackrel{\sim}{\rightarrow} \mathrm{ff} R \operatorname{Aut}(y) \text {-Circles. }
$$

It is given by the natural equivalence $R_{y, w}$ and has the natural equivalence $I_{y, w}$ as inverse, where $R_{y, w}$ and $I_{y, w}$ are defined in the proof using restriction and induction. 
Remark 6.1.2. We easily see that we have an equivalence of categories

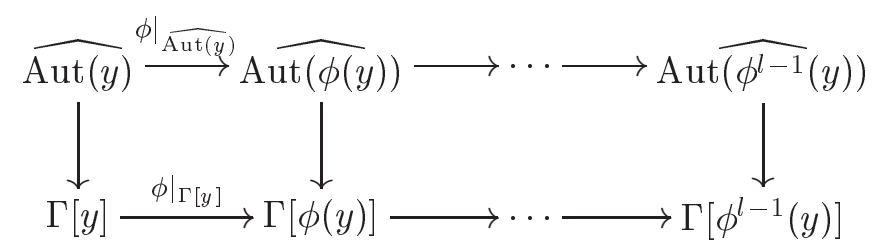

given by the inclusions, but unfortunately, in the diagram

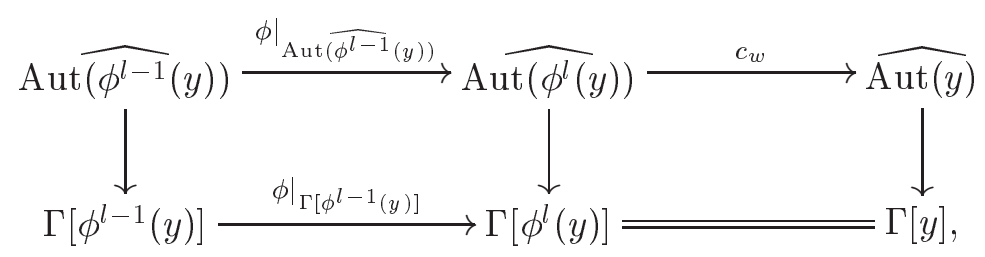

the right square does not commute. If it did, our assertion would be easy to prove, but as it is, we have to work with the modules directly.

We will first prove the following proposition since it contains all the necessary arguments. The general case needs more notation, but not more ideas.

Proposition 6.1.3. Let an object $y \in \Gamma$ with $\overline{\phi(y)}=\bar{y}$ be given, i.e., the corresponding $T$ is $\Gamma[y]$, so $T$ is a connected groupoid. Let $w \in R \Gamma(\phi(y), y)=$ $R T\left(\phi_{T}(y), y\right)$ be a morphism. Then there is an equivalence of categories

$$
R_{y, w}: \phi_{T}-\operatorname{end}_{\mathrm{ff} R T} \stackrel{\sim}{\rightarrow} \phi_{y, w} \text {-end } \operatorname{eff}_{\mathrm{fAut}}(y)
$$

with inverse

$$
I_{y, w}: \phi_{y, w}-\operatorname{end}_{\mathrm{ff} R \operatorname{Aut}(y)} \stackrel{\sim}{\rightarrow} \phi_{T}-\operatorname{end}_{\mathrm{ff} R T} .
$$

If $\Gamma$ is a groupoid with endofunctor $\phi$, in our new notation we write $\phi$-end $\mathrm{dff}_{R \Gamma}$ as $\mathrm{ff} R \Gamma$-Circles. So this proposition follows as a corollary from the theorem in the case where $\overline{\phi(y)}=\bar{y}$.

Proof. Fist we need to define the functors $R_{y, w}$ and $I_{y, w}$. We will use induction and restriction with the equivalence of categories $\operatorname{incl}_{y}$ : $\operatorname{Aut}(y) \rightarrow T$. (We tacitly read $\operatorname{Aut}(y)$ to be $\widehat{\operatorname{Aut}(y)}$.) For better readability, we write ind $y$ and res $y$ instead of ind $_{\text {incl }_{y}}$ and resincl $_{y}$ from now on. We also write $\phi$ instead of $\phi_{T}$ when no confusion can occur. We have a commutative diagram of categories and functors

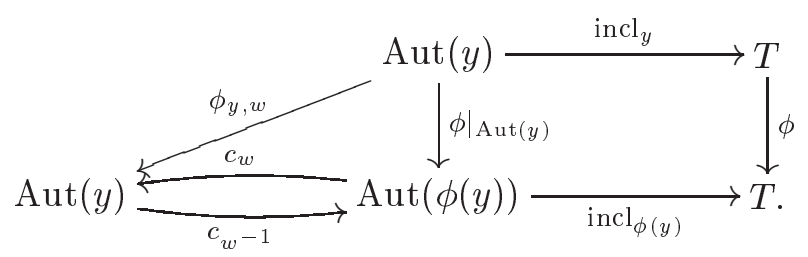


For $M \in \mathrm{ff} R T$ there is a canonical isomorphism of $R \operatorname{Aut}(y)$-modules

$$
\text { canresiso: } \begin{aligned}
\operatorname{res}_{c_{w-1}} \operatorname{res}_{\phi(y)} M & =M(?) \otimes_{R T} R T\left(\operatorname{incl}_{\phi(y)}\left(c_{w^{-1}}(? ?)\right), ?\right) \\
& =M(?) \otimes_{R T} R T\left(c_{w^{-1}} \operatorname{incl}_{y}(? ?), ?\right) \\
& \sim \operatorname{id}_{M} \otimes(-) \circ w^{-1} \\
& =\operatorname{res}_{y} M .
\end{aligned}
$$

The $\operatorname{map} \operatorname{id}_{M} \otimes(-) \circ w^{-1}$ is compatible with the tensor product relation since $\left(v\left(w^{-1} \gamma w\right)\right) w^{-1}=\left(v w^{-1}\right) \gamma$. We define the functor

$$
R_{y, w}: \phi-\operatorname{end}_{\mathrm{ff} R T} \stackrel{\sim}{\rightarrow} \phi_{y, w}-\operatorname{end}_{\mathrm{ff} R \operatorname{Aut}(y)}
$$

on objects $(M \stackrel{g}{\rightarrow} M \circ \phi)$ by the following composition:

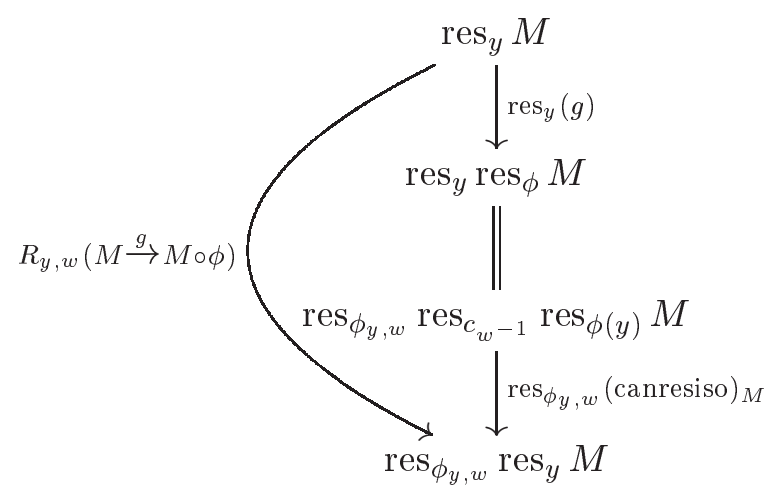

On morphisms, we define it by $R_{y, w}(h):=\operatorname{res}_{y}(h)$. The functor

$$
I_{y, w}: \phi_{y, w}-\operatorname{end}_{\mathrm{ffRAut}(y)} \rightarrow \phi-\operatorname{end}_{\mathrm{ff} R T}
$$

is a little more difficult. We define it on objects by the following composition:

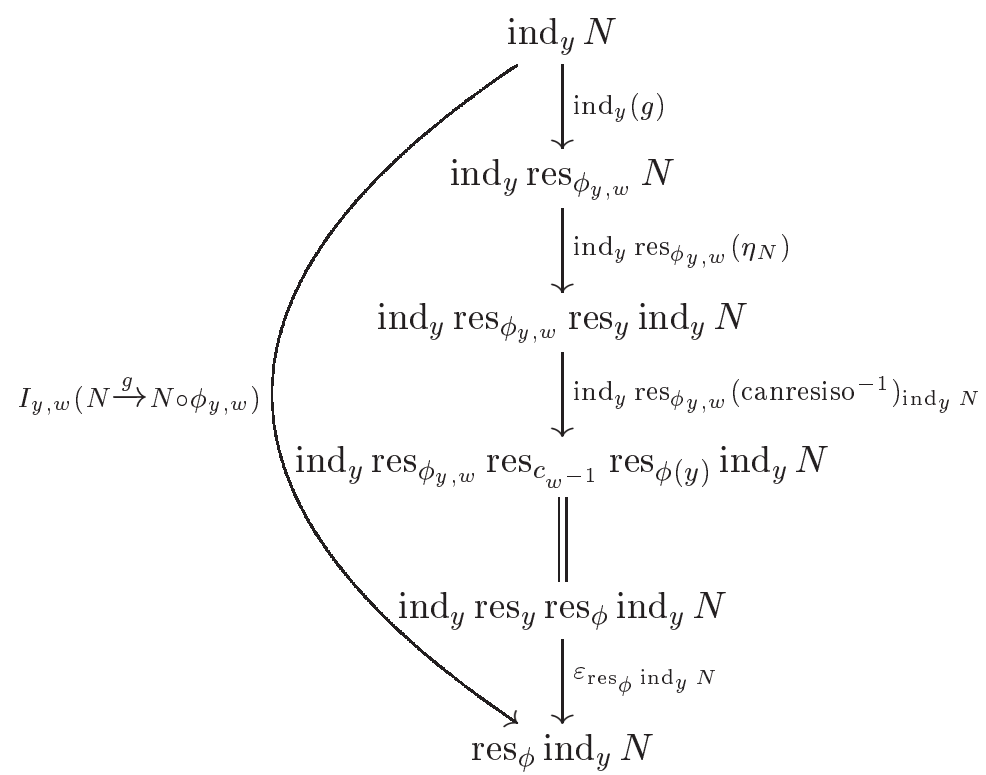


and on morphisms by $I_{y, w}(h):=\operatorname{ind}_{y}(h)$. It is obvious that $R_{y, w}$ and $I_{y, w}$ are functors since on morphisms they are $\operatorname{res}_{y}$ and ind $\mathrm{d}_{y}$ respectively, which are well-known to be functors.

We want to show that the functor $R_{y, w}$ is an equivalence of categories with inverse $I_{y, w}$.

1. There is a natural equivalence $\varepsilon: I_{y, w} \circ R_{y, w} \rightarrow \operatorname{Id}_{\operatorname{Is}\left(\phi-\text { end }_{\mathrm{ff} R T}\right)}$. We know that $I_{y, w} \circ R_{y, w}(M \stackrel{g}{\rightarrow} M \circ \phi)$ is the left vertical composition in the following commutative diagram, where we abbreviate ind by $i$ and res by $r$ :

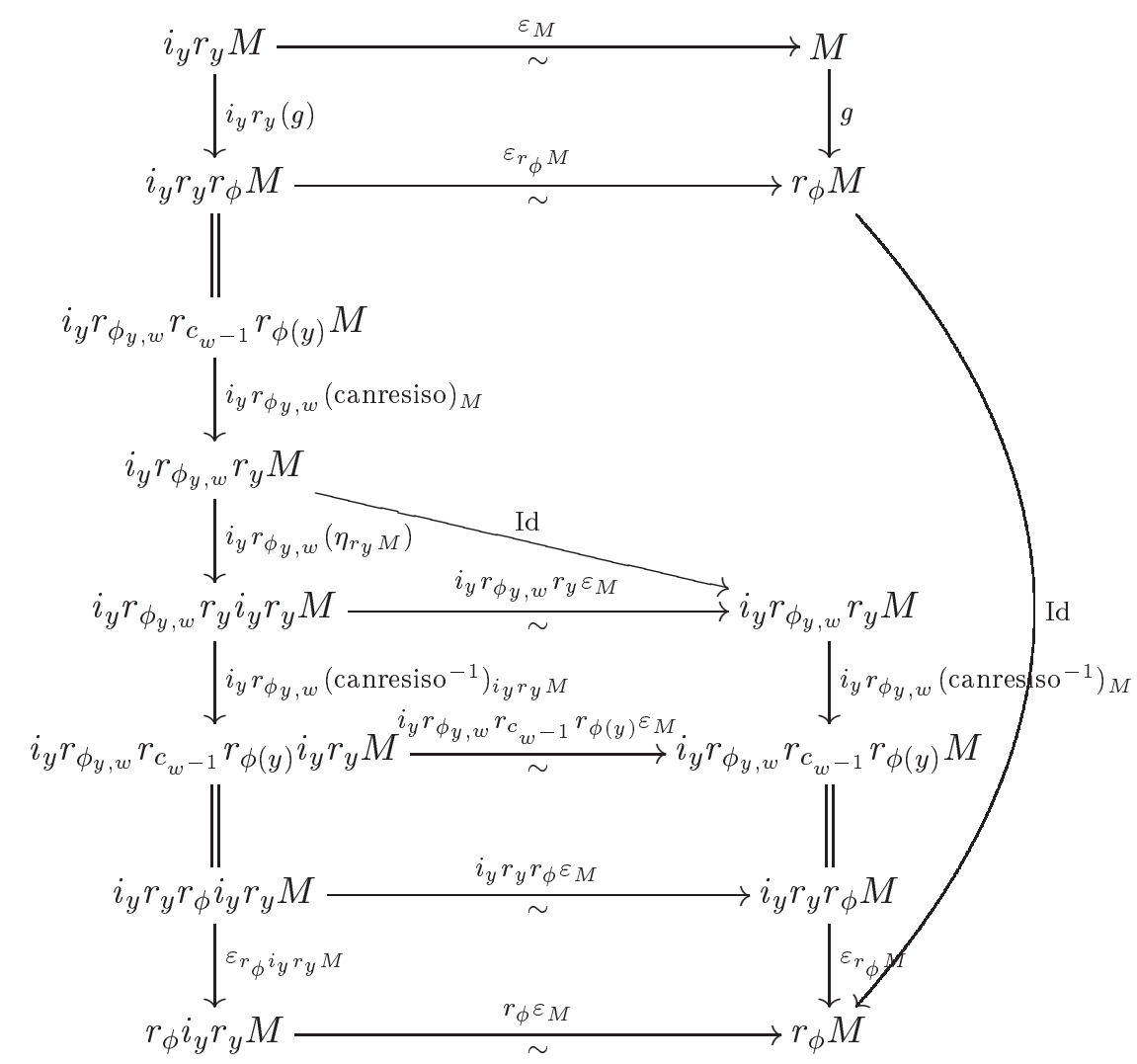

The right vertical composition is $M \stackrel{g}{\rightarrow} M \circ \phi$, and the diagram shows that $\varepsilon_{M}$ is an isomorphism between $I_{y, w} R_{y, w}(M \stackrel{g}{\rightarrow} M \circ \phi)$ and $\operatorname{Id}(M \stackrel{g}{\rightarrow} M \circ \phi)$. We know that $\varepsilon$ is a natural transformation of functors $I_{y, w} R_{y, w} \rightarrow$ Id since on morphisms $h$ we have $I_{y, w} R_{y, w}(h)=$ $\operatorname{ind}_{h} \operatorname{res}_{h}(h)$, and $\varepsilon$ is a natural transformation of functors $\operatorname{ind}_{y} \operatorname{res}_{y} \rightarrow$ Id on $\mathrm{ff} R T$. So $\varepsilon$ is indeed a natural equivalence from $I_{y, w} R_{y, w}$ to Id on $\phi$-end $\mathrm{ff}_{\mathrm{f} T}$.

2. There is a natural equivalence $\eta: R_{y, w} \circ I_{y, w} \rightarrow \operatorname{Id}_{\phi_{y, w}-\operatorname{end}_{\mathrm{ffRAut}(y)}}$. We know that $R_{y, w} \circ I_{y, w}\left(N \stackrel{g}{\rightarrow} N \circ \phi_{y, w}\right)$ is the left vertical composition 
in the following diagram, where we again abbreviate ind by $i$ and res by $r$ :

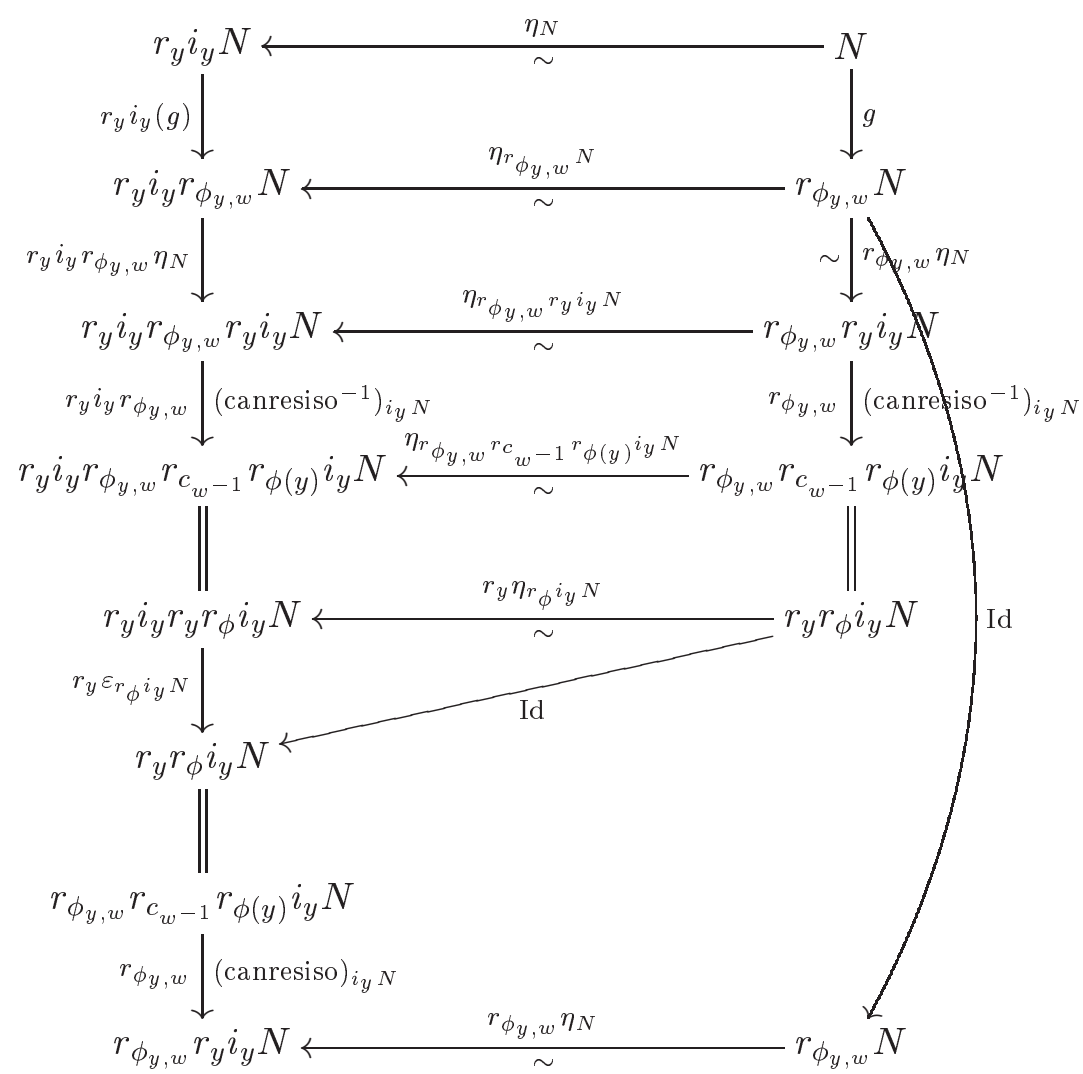

We see that the isomorphism $\eta_{N}$ sends $\left(N \stackrel{g}{\rightarrow} N \circ \phi_{y, w}\right)$ to $R_{y, w} \circ$ $I_{y, w}\left(N \stackrel{g}{\rightarrow} N \circ \phi_{y, w}\right)$. Since on morphisms $h$ we have $R_{y, w} I_{y, w}(h)=$ $\operatorname{res}_{y} \operatorname{ind}_{y}(h)$, and $\eta$ is a natural transformation of functors $\operatorname{res}_{y} \operatorname{ind}_{y} \rightarrow$ Id on $\mathrm{ff} R T$, we know that $\eta$ is a natural transformation from $R_{y, w} I_{y, w}$ to Id on $\phi$-end $\mathrm{ff}_{\mathrm{ff} T}$. Since $\eta_{N}$ is an isomorphism for all $N$, the natural transformation $\eta$ is a natural equivalence.

We have shown that $R_{y, w}$ is an equivalence of categories with inverse $I_{y, w}$. This implies that $K\left(R_{y, w} \circ I_{y, w}\right)=\operatorname{Id}_{K\left(\phi_{y, w}-\operatorname{end}_{\mathrm{ffRAut}(y)}\right)}$ and that $K\left(I_{y, w} \circ\right.$ $\left.R_{y, w}\right)=\operatorname{Id}_{K\left(\phi-\text { end }_{\mathrm{ff} R T}\right)}$. So $K\left(R_{y, w}\right)$ and $K\left(I_{y, w}\right)$ are group homomorphisms inverse to each other.

Instead of the category $\phi$-end $\mathrm{eff}_{\mathrm{f} T}$, we want to use $\phi_{y, w}$-end $\mathrm{enfRAt}_{\mathrm{ff})}$ and look at our invariant in $K_{0}\left(\phi_{y, w}\right.$-end $\left.\mathrm{fffAut}(y)_{\mathrm{f}}\right)$ by applying $K_{0}\left(R_{y, w}\right)$ to it. In order to make the definition independent of the choice of $y$ and $w$, we set

$$
V_{T}:=\coprod_{\substack{y \in T, w \in R T(\phi(y), y)}} K\left(\phi_{y, w}-\operatorname{end}_{\mathrm{ffRAut}(y)}\right) / \sim,
$$


where we identify $a \in K\left(\phi_{y, w}\right.$-end $\left.\operatorname{fff} R \operatorname{Aut}(y)\right)$ and $a^{\prime} \in K\left(\phi_{y^{\prime}, w^{\prime}}\right.$-end ${\operatorname{enfRAut}\left(y^{\prime}\right)}_{\mathrm{f}})$ if and only if $K\left(R_{y^{\prime}, w^{\prime}} \circ I_{y, w}\right)(a)=a^{\prime}$. This is well-defined since $K\left(R_{y, w} \circ\right.$ $\left.I_{y, w}\right)=\operatorname{Id}_{K\left(\phi_{y, w}-\operatorname{end}_{\mathrm{ffRAut}(y)}\right)}$ and $K\left(R_{y^{\prime \prime}, w^{\prime \prime}} \circ I_{y^{\prime}, w^{\prime}}\right) \circ K\left(R_{y^{\prime}, w^{\prime}} \circ I_{y, w}\right)=$ $K\left(R_{y^{\prime \prime}, w^{\prime \prime}}\right) \circ K\left(I_{y^{\prime}, w^{\prime}} \circ R_{y^{\prime}, w^{\prime}}\right) \circ K\left(I_{y, w}\right)=K\left(R_{y^{\prime \prime}, w^{\prime \prime}} \circ I_{y, w}\right)$. So our definition is indeed independent of the choice of $y \in T$ and of $w \in R T(\phi(y), y)$. For any choice of $y$ and $w$, the map $K\left(R_{y, w}\right)$ induces the same isomorphism

$$
\xi_{T}: K\left(\phi_{T}-\operatorname{end}_{\mathrm{ffRT}}\right) \stackrel{\sim}{\rightarrow} V_{T} .
$$

Now we are ready for the general case, i.e., for the proof of Theorem 6.1.1.

Proof (of Theorem 6.1.1). We proceed in analogy to the special case. First we have to define the functor $R_{y, w}$. Let

$$
\left(\left(M_{i}\right)_{0 \leq i \leq l-1},\left(g_{i}\right)_{0 \leq i \leq l-1}\right) \in \mathrm{ff} R \Gamma[y] \text {-Circles. }
$$

We map $M_{i}$ to $\operatorname{res}_{\phi^{i}(y)} M_{i}$ for $0 \leq i \leq l-1$ and $g_{i}$ to $\operatorname{res}_{\phi^{i}(y)} g_{i}$ for $0 \leq i \leq l-2$.

The maps $\left\{g, \ldots, g_{l-2}\right\}$ do not pose any problems since for $0 \leq i \leq l-2$

$$
\begin{aligned}
\operatorname{res}_{\phi^{i}(y)} \operatorname{res}_{\left.\phi\right|_{\Gamma\left[\phi^{i}(y)\right]}} M_{i+1} & =\operatorname{res}_{\left.\phi\right|_{\Gamma\left[\phi^{i}(y)\right]}} \operatorname{incl}_{\phi^{i}(y)} M_{i+1} \\
& =\operatorname{res}_{\text {incl }\left._{\phi^{i+1}(y)} \circ \phi\right|_{\operatorname{Aut}\left(\phi^{i}(y)\right)}} M_{i+1} \\
& =\operatorname{res}_{\left.\phi\right|_{\operatorname{Aut}\left(\phi^{i}(y)\right)}} \operatorname{res}_{\phi^{i+1}(y)} M_{i+1},
\end{aligned}
$$

$\operatorname{sores}_{\phi^{i}(y)} g_{i}: \operatorname{res}_{\phi^{i}(y)} M_{i} \rightarrow \operatorname{res}_{\phi^{i}(y)} \operatorname{res}_{\left.\phi\right|_{\Gamma\left[\phi^{i}(y)\right]}} M_{i+1}=\operatorname{res}_{\left.\phi\right|_{\text {Aut }\left(\phi^{i}(y)\right)}} \operatorname{res}_{\phi^{i+1}(y)} M_{i+1}$ has the desired form. We have to be careful with $g_{l-1}$ since $\operatorname{res}_{\phi^{l-1}(y)}\left(g_{l-1}\right)$ does not map quite to the right module: We have

$$
\begin{aligned}
\operatorname{res}_{\phi^{l-1}(y)}\left(g^{l-1}\right): \operatorname{res}_{\phi^{l-1}(y)} M_{l-1} \rightarrow & \operatorname{res}_{\phi^{l-1}(y)} \operatorname{res}_{\left.\phi\right|_{\Gamma\left[\phi^{l-1}(y)\right]}} M_{0} \\
& =\operatorname{res}_{\left.\phi\right|_{A u t\left(\phi^{l-1}(y)\right)}} \operatorname{res}_{\phi^{l}(y)} M_{0} \\
& =\operatorname{res}_{\phi_{y, w}} \operatorname{res}_{c_{w}-1} \operatorname{res}_{\phi^{l}(y)} M_{0} .
\end{aligned}
$$

We again use the canonical isomorphism

$$
\text { canresiso: } \operatorname{res}_{c_{w^{-1}}} \operatorname{res}_{\phi^{l}(y)} M_{0} \rightarrow \operatorname{res}_{y} M_{0}
$$

and compose the above with $\operatorname{res}_{\phi_{y, w}}$ (canresiso), i.e., we map $g_{l-1}: M_{l-1} \rightarrow$ $\operatorname{res}_{\left.\phi\right|_{\Gamma\left[\phi^{l-1}(y)\right]}} M_{0}$ to $\operatorname{res}_{\phi_{y, w}}($ canresiso $) \circ \operatorname{res}_{\phi^{l-1}(y)}\left(g^{l-1}\right)$. So we have defined $R_{y, w}$ by

$$
\begin{aligned}
R_{y, w}: \operatorname{ff} R \Gamma[y] \text {-Circles } \rightarrow & \operatorname{ffRAut}(y) \text {-Circles } \\
\left(\left(M_{i}\right)_{0 \leq i \leq l-1},\left(g_{i}\right)_{0 \leq i \leq l-1}\right) \mapsto & \left(\left(\operatorname{res}_{\phi^{i}(y)} M_{i}\right)_{0 \leq i \leq l-1},\right. \\
& \left(\operatorname{res}_{\phi^{0}(y)}\left(g_{0}\right), \ldots, \operatorname{res}_{\phi^{l-2}(y)}\left(g_{l-2}\right),\right. \\
& \left.\left.\operatorname{res}_{\phi_{y, w}}(\text { canresiso }) \circ \operatorname{res}_{\phi^{l-1}(y)}\left(g_{l-1}\right)\right)\right) .
\end{aligned}
$$


As in the special case, the definition of $I_{y, w}$ is a little more complicated. Let

$$
\left(\left(N_{i}\right)_{0 \leq i \leq l-1},\left(k_{i}\right)_{0 \leq i \leq l-1}\right) \in \operatorname{ffRAut}(y) \text {-Circles. }
$$

We map $N_{i}$ to $\operatorname{ind}_{\phi^{i}(y)} N_{i}$ for $0 \leq i \leq l-1$. We cannot map $k_{i}$ to $\operatorname{ind}_{\phi^{i}(y)} k_{i}$ for $0 \leq i \leq l-2$ since this morphism does not land in the right module. Instead, we have to compose it with the composition

$$
\begin{array}{r}
\left(\left(\varepsilon_{i}\right)_{\left.\operatorname{res}_{\phi \mid}\right|_{\Gamma\left[\phi^{i}(y)\right]}} \operatorname{ind}_{\phi^{i+1}(y)} N_{i+1}\right) \circ\left(\operatorname{ind}_{\phi^{i}(y)} \operatorname{res}_{\left.\phi\right|_{\operatorname{Aut}\left(\phi^{i}(y)\right)}}\left(\eta_{i+1}\right)_{N_{i+1}}\right): \\
\operatorname{ind}_{\phi^{i}(y)} \operatorname{res}_{\left.\phi\right|_{\operatorname{Aut}\left(\phi^{i}(y)\right)}} N_{i+1} \rightarrow \operatorname{res}_{\left.\phi\right|_{\Gamma\left[\phi^{i}(y)\right]}} \operatorname{ind}_{\phi^{i+1}(y)} N_{i+1}
\end{array}
$$

to obtain the desired map given by $\left(k_{i}\right)_{*}:=\left(\left(\varepsilon_{i}\right)_{\operatorname{res}_{\phi \mid}\left[\phi^{i}(y)\right]} \operatorname{ind}_{\phi^{i+1}(y)} N_{i+1}\right) \circ$ $\left.\operatorname{(ind}_{\phi^{i}(y)} \operatorname{res}_{\left.\phi\right|_{\text {Aut }\left(\phi^{i}(y)\right)}}\left(\eta_{i+1}\right)_{N_{i+1}}\right) \circ \operatorname{ind}_{\phi^{i}(y)}\left(k_{i}\right)$. This is analogous to the definition of $H_{*}$ in Section 2.2. In order to treat $k_{l-1}$, just like in the special case we use $\left(\right.$ canresiso $\left.^{-1}\right)$ and map $k_{l-1}$ to the composition $\left(\left(\varepsilon_{l-1}\right)_{\operatorname{res}_{\phi \mid}\left[\phi^{l-1}(y)\right]} \operatorname{ind}_{y} N_{0}\right) \circ$ $\left(\operatorname{ind}_{\phi^{l-1}(y)} \operatorname{res}_{\phi_{y, w}}\left(\operatorname{canresiso}^{-1}\right)_{\operatorname{ind}_{y} N}\right) \circ\left(\operatorname{ind}_{\phi^{l-1}(y)} \operatorname{res}_{\phi_{y, w}}\left(\eta_{0}\right)_{N_{0}}\right) \circ \operatorname{ind}_{\phi^{l-1}(y)}\left(k_{l-1}\right)$. Here we used the notations $\varepsilon_{i}, \eta_{i+1}$ etc. to make clear which adjunction morphisms we mean, but we will mostly omit the indices in the sequel.

We show that $R_{y, w}$ is an equivalence of categories with inverse $I_{y, w}$.

We will show that there is a natural equivalence between $I_{y, w} \circ R_{y, w}$ and Id given by a natural transformation $\varepsilon$. This natural transformation $\varepsilon$ is defined to be the $l$-tuple $\left(\varepsilon_{0}, \ldots, \varepsilon_{l-1}\right)$, where $\varepsilon_{j}$ is the natural transformation defined by the adjointness of $\operatorname{ind}_{\phi^{j}(y)}$ and $\operatorname{res}_{\phi^{j}(y)}$.

Let $\left(\left(M_{j}\right),\left(g_{j}\right)\right) \in \mathrm{ff} R T$-Circles. On the modules $M_{j}$, for $0 \leq j \leq l-1$, the image under $I_{y, w} \circ R_{y, w}$ is given by $\operatorname{ind}_{\phi^{j}(y)} \operatorname{res}_{\phi^{j}(y)} M_{j}$, and this is mapped to $M_{j}$ by $\varepsilon_{j}$ as desired.

On the morphisms $g_{j}$, for $0 \leq j \leq l-2$, the image under $I_{y, w} \circ R_{y, w}$ is given by the left vertical composition in the following commutative diagram, from which we see that again $\varepsilon$ gives a natural transformation to the identity. 
We again abbreviate ind by $i$ and res by $r$.

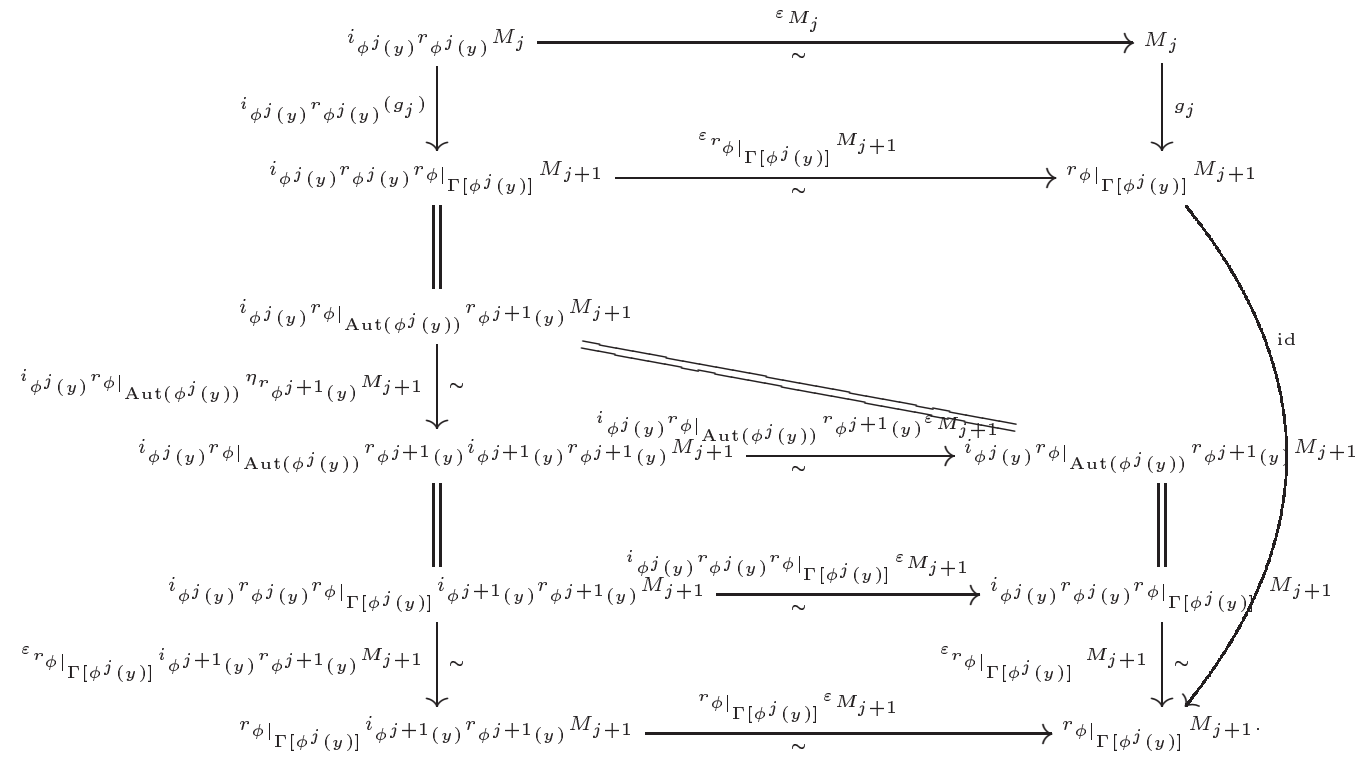

Finally, we have to treat the case of $g_{l-1}$. As in the special case, $\varepsilon$ is again a natural transformation from $I_{y, w} \circ R_{y, w}$ to Id, the appropriate diagram can be found in Proposition 6.1.3, part 1 . So we have seen that $\left(\varepsilon_{0}, \ldots, \varepsilon_{l-1}\right)$ is the desired natural equivalence from $I_{y, w} \circ R_{y, w}$ to Id.

There is a natural equivalence between Id and $R_{y, w} \circ I_{y, w}$ given by a natural transformation $\eta$. This natural transformation $\eta$ is defined to be the $l$-tuple $\left(\eta_{0}, \ldots, \eta_{l-1}\right)$, where $\eta_{j}$ is the natural transformation defined by the adjointness of ind $\phi_{\phi^{j}(y)}$ and $\operatorname{res}_{\phi^{j}(y)}$. Let $\left(\left(N_{j}\right),\left(k_{j}\right)\right) \in \operatorname{ffRAut}(y)$-Circles. On the modules $N_{j}$, for $0 \leq j \leq l-1$, the image under $R_{y, w} \circ I_{y, w}$ is given by $\operatorname{res}_{\phi^{j}(y)} \operatorname{ind}_{\phi^{j}(y)} N_{j}$, and we know that $\eta_{j}$ is a natural equivalence from $N_{j}$ to $\operatorname{res}_{\phi^{j}(y)} \operatorname{ind}_{\phi^{j}(y)} N_{j}$. On the morphisms $k_{j}$, for $0 \leq j \leq l-2$, the image under $R_{y, w} \circ I_{y, w}$ is given by the left vertical composition in the following 
commutative diagram. We again abbreviate ind by $i$ and res by $r$.

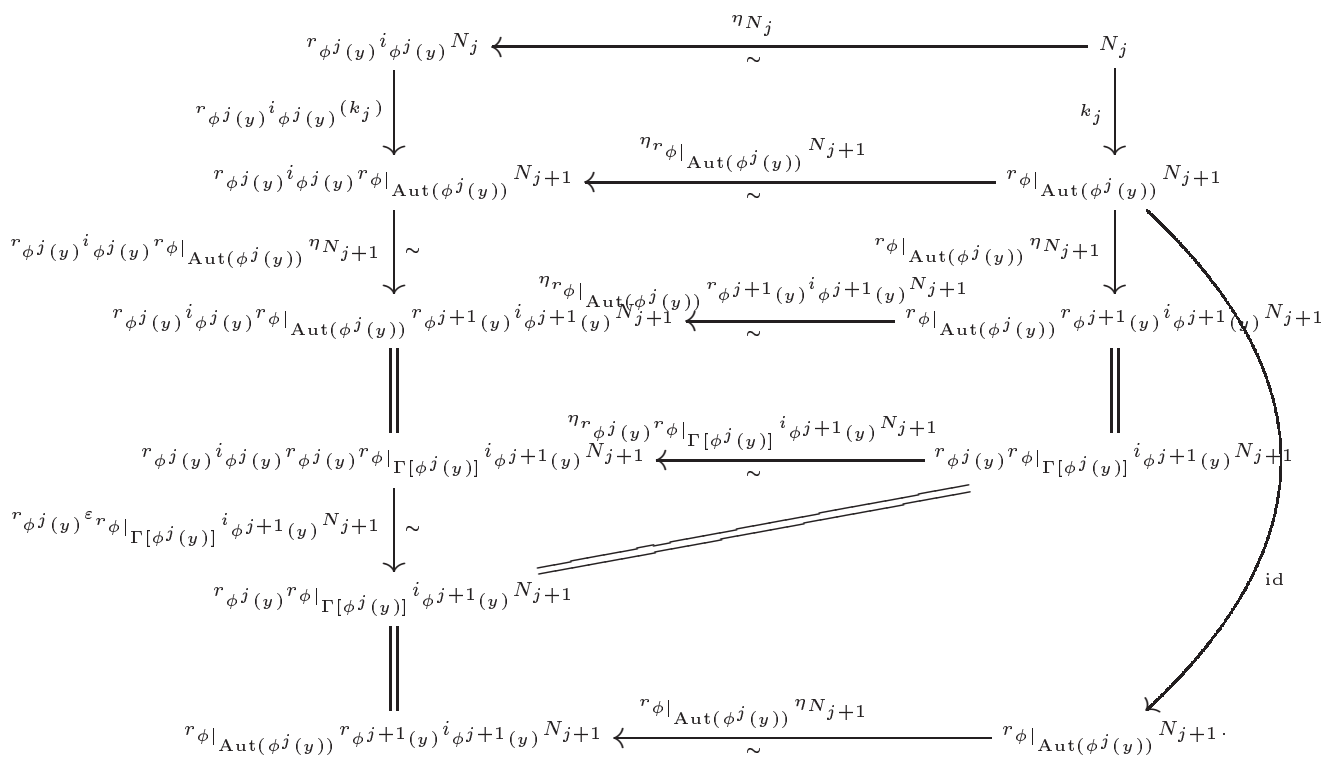

We see that $\eta$ gives a natural transformation from Id to this composition. Finally, for $k_{l-1}$ we use the diagram from the special case Proposition 6.1.3, part 2, to show that $\eta$ is a natural transformation from Id to $R_{y, w} \circ I_{y, w}$. So we see that $\left(\eta_{0}, \ldots, \eta_{l-1}\right)$ is the desired natural equivalence from Id to $R_{y, w} \circ I_{y, w}$.

As in the special case, we have shown that $R_{y, w}$ is an equivalence of categories with inverse $I_{y, w}$ and can conclude that $K\left(R_{y, w}\right)$ and $K\left(I_{y, w}\right)$ are group homomorphisms inverse to each other. As before, instead of the category ff $R T$-Circles, we want to use the category $\operatorname{ff} R \operatorname{Aut}(y)$-Circles and look at our invariant in $K_{0}\left(\mathrm{ff} R \operatorname{Aut}(y)\right.$-Circles) by applying $K_{0}\left(R_{y, w}\right)$ to it. In order to make the definition independent of the choices, we set

$$
V_{T_{i}}:=\coprod_{\substack{y \in T_{i}, w \in R T_{i}\left(\phi^{l} i(y), y\right)}} K(\operatorname{ffRAut}(y) \text {-Circles }) / \sim,
$$

where for $a \in K(\mathrm{ffRAut}(y)$-Circles $)$ and $a^{\prime} \in K\left(\mathrm{ff} R \operatorname{Aut}\left(y^{\prime}\right)\right.$-Circles $)$ we have

$$
a \sim a^{\prime} \Leftrightarrow K\left(R_{y^{\prime}, w^{\prime}} \circ I_{y, w}\right)(a)=a^{\prime} .
$$

This is well-defined since $K\left(R_{y, w} \circ I_{y, w}\right)=\operatorname{Id}_{K\left(\mathrm{ff} R \operatorname{Aut}\left(x_{i j}\right)\right)}$ by the existence of the natural equivalence $\eta$ and $K\left(R_{y^{\prime \prime}, w^{\prime \prime}} \circ I_{y^{\prime}, w^{\prime}} \circ R_{y^{\prime}, w^{\prime}} \circ I_{y, w}\right)=K\left(R_{y^{\prime \prime}, w^{\prime \prime}} \circ\right.$ $\left.I_{y, w}\right)$ because we have $K\left(I_{y^{\prime}, w^{\prime}} \circ R_{y^{\prime}, w^{\prime}}\right)=$ Id by the existence of the natural equivalence $\varepsilon$. We have obtained a "basepoint free" description of our groups in terms of groups and group homomorphisms instead of groupoids and functors between them. 
As in the special case, for any choice of $y$ and $w$ the map $K\left(R_{y, w}\right)$ induces the same isomorphism

$$
\xi_{T}: K\left(\phi_{T}-\operatorname{end}_{\mathrm{ffRT}}\right) \stackrel{\sim}{\rightarrow} V_{T} .
$$

We set $U_{T}:=V_{0 T} \cong K_{0}\left(\phi_{T}-\operatorname{end}_{\mathrm{ff} R T}\right)$ and $U_{Q}:=V_{0 Q} \cong K_{0}($ ff $R Q)$. From Corollary 5.4.4 we conclude the following corollary.

Corollary 6.1.4. We have an isomorphism

$$
\bigoplus_{W_{i} \in I_{\Gamma}}\left(\xi_{T_{i}} K\left(S_{T_{i}}\right) \oplus \xi_{Q_{i}} K\left(\left.S_{Q_{i}}\right|_{\mathrm{Mod}}\right)\right): K\left(\phi-\operatorname{end}_{\mathrm{ff} R \Gamma}\right) \stackrel{\sim}{\rightarrow} \bigoplus_{W_{i} \in I_{\Gamma}}\left(V_{T_{i}} \oplus V_{Q_{i}}\right)
$$

with inverse $\bigoplus_{W_{i} \in I_{\Gamma}} K\left(E_{T_{i}} I_{x_{i}, w_{i}}\right) \oplus K\left(E_{Q_{i}} \operatorname{iom} \oplus_{j \in J_{i}} \operatorname{ind}_{y_{i j}}\right)$ for any $x_{i} \in T_{i}$, $w \in \operatorname{Mor}\left(f^{l_{i}}\left(x_{i}\right), x_{i}\right)$ and $y_{i j} \in Q_{i j}$.

This is a basepoint free description of the splitting result in terms of groups instead of groupoids.

\subsection{The Geometric Meaning of the Splitting Re- sult}

In the geometric setting, we are given a discrete group $G$, a finite proper $G$-CW-complex $X$ and a $G$-equivariant endomorphism $f: X \rightarrow X$. In Chapter 3 we introduced the universal functorial equivariant Lefschetz invariant $\left(U_{G}^{\mathbb{Z}}(X, f), u_{G}^{\mathbb{Z}}(X, f)\right)$, where

$$
\begin{aligned}
& U_{G}^{\mathbb{Z}}(X, f):=U(\mathbb{Z}, \Gamma(G, X), \phi):=K_{0}\left(\phi-\operatorname{end}_{\mathrm{ff} \mathbb{Z} \Pi(G, X)}\right) \\
& u_{G}^{\mathbb{Z}}(X, f):=u\left(C^{c}(\widetilde{f})\right) \in U_{G}^{\mathbb{Z}}(X, f) .
\end{aligned}
$$

We now discuss what the splitting results mean for this geometric invariant. As seen in Corollary 5.4.4, we have an algebraic splitting isomorphism. It is given by

$$
\begin{aligned}
& \bigoplus_{W_{i} \in I_{\Pi(G, X)}} K\left(S_{T_{i}}\right) \oplus K\left(\left.S_{Q_{i}}\right|_{\mathrm{Mod}}\right): \\
& K\left(\phi-\operatorname{end}_{\mathrm{ff \mathbb {Z }} \Pi(G, X)}\right) \stackrel{\sim}{\rightarrow} \bigoplus_{W_{i} \in I_{\Pi(G, X)}}\left(K\left(\left.\phi\right|_{T_{i}}-\operatorname{end}_{\mathrm{ff} \mathbb{Z} T_{i}}\right) \oplus K\left(\mathrm{ff} \mathbb{Z} Q_{i}\right)\right) .
\end{aligned}
$$

The set $I_{\Pi(G, X)}$ is the set of orbits in Is $\Pi(G, X)$ under $\phi$, where $\phi:=\Pi(G, f)$ is induced by composition with $f$, as defined in Definition 5.1.1. We know that Is $\Pi(G, X)=\coprod_{(H) \in \operatorname{consub} G} W H \backslash \pi_{0}\left(X^{H}\right)$, where consub $G$ denotes the set of conjugacy classes of subgroups of $G$. Since $\phi$ is induced by composition with $f$, it respects the decomposition indexed by $(H) \in \operatorname{consub} G$. 
If $W H \backslash \pi_{0}\left(X^{H}\right)$ is partitioned into $n_{H}$ subsets $\left\{W_{i}^{H}\right\}_{i \in\left\{1, \ldots, n^{H}\right\}}$ which are $\phi$-invariant, then we obtain

$$
I_{\Pi(G, X)}=\coprod_{(H) \in \text { consub } G} \coprod_{i=1}^{n_{H}} T_{i}^{H} \amalg Q_{i}^{H} .
$$

We choose elements $y_{i j}^{H} \in Q_{i j}^{H}, x_{i}^{H} \in T_{i}^{H}$ and $w_{i}^{H} \in R T_{i}^{H}\left(\phi^{l_{i}}\left(x_{i}^{H}\right), x_{i}^{H}\right)$, where $l_{i}:=l\left(T_{i}\right)$ is the length of $T_{i}$ defined in Definition 5.4.1. Remember that in the category $\Pi(G, X)$ a path has the form $w=(\sigma,[v]) \in \operatorname{Mor}(x, y)$, where $\sigma: G / H \rightarrow G / K$ and $v: G / H \times I \rightarrow X$ with $v_{1}=x$ and $v_{0}=y \circ \sigma$. By Section 6.1, the choice of elements does not play a role. We have

$$
\begin{aligned}
& T_{i}^{H}=W H \cdot X^{H}\left(x_{i}^{H}\right) \amalg W H \cdot X^{H}\left(f\left(x_{i}^{H}\right)\right) \amalg \cdots \amalg W H \cdot X^{H}\left(f^{l_{i}-1}\left(x_{i}^{H}\right)\right), \\
& Q_{i}^{H}=\coprod_{j \in J_{i}^{H}} W H \cdot X^{H}\left(y_{i j}^{H}\right) .
\end{aligned}
$$

We state the geometric interpretation of the splitting results in the following theorem.

Theorem 6.2.1. Let $G$ be a discrete group, let $X$ be a finite proper $G$ $C W$-complex and let $f: X \rightarrow X$ be a $G$-equivariant endomorphism. Let $\left(U_{G}^{\mathbb{Z}}(X, f), u_{G}^{\mathbb{Z}}(X, f)\right)$ be the universal functorial Lefschetz invariant of $(X, f)$. With the notation given above, choosing elements $y_{i j}^{H} \in Q_{i j}^{H}, x_{i}^{H} \in T_{i}^{H}$ and $w_{i}^{H} \in \operatorname{Mor}\left(\phi^{l_{i}}\left(x_{i}^{H}\right), x_{i}^{H}\right)$, there is an isomorphism

$$
\begin{aligned}
& \zeta:=\bigoplus_{(H) \in \operatorname{consub} G} \bigoplus_{i=1}^{n_{H}}\left(K_{0}\left(R_{x_{i}^{H}, w_{i}^{H}} S_{T_{i}^{H}}\right) \oplus K_{0}\left(\left.\operatorname{res}_{y_{i j}^{H}} S_{Q_{i}}\right|_{\text {Mod }}\right)\right): \\
& U_{G}^{\mathbb{Z}}(X, f) \stackrel{\sim}{\rightarrow} \bigoplus_{(H) \in \text { consub } G} \bigoplus_{i=1}^{n_{H}}\left(K_{0}\left(\operatorname{ff} \mathbb{Z} \operatorname{Aut}\left(x_{i}^{H}\right) \text {-Circles }\right) \oplus \bigoplus_{j \in J_{i}^{H}} K_{0}\left(\operatorname{ff} \mathbb{Z} \operatorname{Aut}\left(y_{i j}^{H}\right)\right)\right) .
\end{aligned}
$$

Under this isomorphism,

$$
u_{G}^{\mathbb{Z}}(X, f) \mapsto \sum_{(H) \in \text { consub }} \sum_{i=1}^{n_{H}}\left(u_{G}^{\mathbb{Z}}(X, f)_{x_{i}}+\sum_{j \in J_{i}^{H}} u_{G}^{\mathbb{Z}}(X, f)_{y_{i j}}\right),
$$

where

$$
\begin{aligned}
u_{G}^{\mathbb{Z}}(X, f)_{x_{i}}= & {\left[\left(C^{c}\left(\left.\widetilde{f}\right|_{\widetilde{X}^{H}\left(x_{i}^{H}\right)},\left.\widetilde{f}\right|_{\tilde{X}^{>H}\left(x_{i}^{H}\right)}\right), \ldots,\right.\right.} \\
& \left.\left.C^{c}\left(\left.\widetilde{g_{w_{i}}^{-1} f}\right|_{\widetilde{X}^{H}\left(f^{l_{i}-1}\left(x_{i}^{H}\right)\right)},\left.{\hat{w_{i}}}_{-1}\right|_{\widetilde{X}^{>H}\left(f^{l_{i}-1}\left(x_{i}^{H}\right)\right)}\right)\right)\right], \\
u_{G}^{R}(X, f)_{y_{i j}}= & {\left[C^{c}\left(\widetilde{X}^{H}\left(y_{i j}^{H}\right), \widetilde{X}^{>H}\left(y_{i j}^{H}\right)\right)\right] . }
\end{aligned}
$$

The element $g_{w_{i}}$ is determined by the morphism $w_{i}^{H}=\left(\sigma_{i}^{H},\left[v_{i}^{H}\right]\right)$. One chooses an element $g_{w_{i}} \in N_{G} H$ such that $\sigma_{i}^{H}=R_{g_{w_{i}}}: G / H \rightarrow G / H, g^{\prime} H \mapsto$ $g^{\prime} g_{w_{i}} H$. 
For better readability, we left out the modules in the equation describing $u_{G}^{\mathbb{Z}}(X, f)_{x_{i}}$. The element $g_{w_{i}}$ is only well-defined up to a factor in $H$, but this does not affect the map defined on a subset of the fixed point set $X^{H}$.

Proof. The restriction maps $R_{x_{i}^{H}, w_{i}^{H}}$ and $\operatorname{res}_{y_{i j}^{H}}$ induce isomorphisms

$$
\begin{array}{rlrl}
K_{0}\left(R_{x_{i}^{H}, w_{i}^{H}}\right): K_{0}\left(\left.\phi\right|_{T_{i}}-\operatorname{end}_{\mathrm{ff} \mathbb{Z} T_{i}}\right) & \stackrel{\sim}{\rightarrow} K_{0}\left(\mathrm{ff} \mathbb{Z} \operatorname{Aut}\left(x_{i}^{H}\right) \text {-Circles }\right) \\
K_{0}\left(\operatorname{res}_{y_{i j}^{H}}\right): & K_{0}\left(\operatorname{ff} \mathbb{Z} Q_{i j}^{H}\right) & \stackrel{\sim}{\rightarrow} K_{0}\left(\operatorname{ff} \mathbb{Z} \operatorname{Aut}\left(y_{i j}^{H}\right)\right) .
\end{array}
$$

Combining these with the isomorphism given in Corollary 5.4.4, we obtain an isomorphism

$$
\begin{aligned}
& \bigoplus_{(H) \in \operatorname{consub} G} \bigoplus_{i=1}^{n_{H}}\left(K_{0}\left(R_{x_{i}^{H}, w_{i}^{H}} S_{T_{i}^{H}}\right) \oplus K_{0}\left(\left.\operatorname{res}_{y_{i j}^{H}} S_{Q_{i}}\right|_{\text {Mod }}\right)\right): \\
& K_{0}\left(\phi-\operatorname{end}_{\mathrm{fffZ} \Pi(G, X)}\right) \stackrel{\sim}{\rightarrow} \\
& \bigoplus_{(H) \in \operatorname{consub} G} \bigoplus_{i=1}^{n_{H}}\left(K_{0}\left(\operatorname{ff} \mathbb{Z} \operatorname{Aut}\left(x_{i}^{H}\right) \text {-Circles }\right) \oplus \underset{j \in J_{i}^{H}}{\bigoplus} K_{0}\left(\operatorname{ff} \mathbb{Z} \operatorname{Aut}\left(y_{i j}^{H}\right)\right)\right) .
\end{aligned}
$$

Under the above isomorphism, the invariant

$$
u_{G}^{\mathbb{Z}}(X, f):=\left[C^{c}(\widetilde{f}): C^{c}(\widetilde{X}) \rightarrow C^{c}(\widetilde{X}) \circ \phi\right]
$$

is mapped to

$$
\sum_{(H) \in \operatorname{consub} G} \sum_{i=1}^{n_{H}}\left(\left[R_{x_{i}^{H}, w_{i}^{H}} S_{T_{i}^{H}}\left(C^{c}(\widetilde{f})\right)\right]+\sum_{j \in J_{i}^{H}}\left[S_{y_{i j}^{H}}\left(C^{c}(\widetilde{X})\right)\right]\right) .
$$

There is a natural isomorphism of $\mathbb{Z} \operatorname{Aut}(x)$-chain complexes

$$
S_{x}\left(C^{c}(\widetilde{X})\right) \cong C^{c}\left(\widetilde{X}^{H}(x), \widetilde{X}^{>H}(x)\right)
$$

where $(x: G / H \rightarrow X) \in \mathrm{Ob}(\Pi(G, X))$ [Lüc89, Lemma 9.32]. So the $Q_{i j^{-}}$ part becomes

$$
\left[S_{y_{i j}^{H}}\left(C^{c}(\widetilde{X})\right)\right]=\left[C^{c}\left(\widetilde{X}^{H}\left(y_{i j}^{H}\right), \widetilde{X}^{>H}\left(y_{i j}^{H}\right)\right)\right] \in K_{0}\left(\operatorname{ff} \mathbb{Z} \operatorname{Aut}\left(y_{i j}^{H}\right)\right) .
$$

What about the $T_{i}$-part? For ease of notation, we write $x, w, T$ and $l$ instead of $x_{i}^{H}, w_{i}^{H}, T_{i}^{H}$ and $l_{i}$ since no confusion can occur. We know that $R_{x, w} S_{T}\left(C^{c}(\widetilde{f})\right)$ is an element $\left(\left(M_{0}, \ldots, M_{l-1}\right),\left(g_{0}, \ldots, g_{l-1}\right)\right)$ in the category ffZZAut $(x)$-Circles. The modules are

$$
M_{i}=\operatorname{res}_{f^{i}(x)} S_{T}\left(C^{c}(\widetilde{X})\right) \cong S_{f^{i}(x)}\left(C^{c}(\widetilde{X})\right) \cong C^{c}\left(\widetilde{X}^{H}\left(f^{i}(x)\right), \tilde{X}^{>H}\left(f^{i}(x)\right)\right) .
$$


For $0 \leq i \leq l-2$, the morphisms are $g_{i}=\operatorname{res}_{f^{i}(x)} S_{T}\left(C^{c}(\widetilde{f})\right)$, where $S_{T}\left(C^{c}(\widetilde{f})\right)$ is the composition

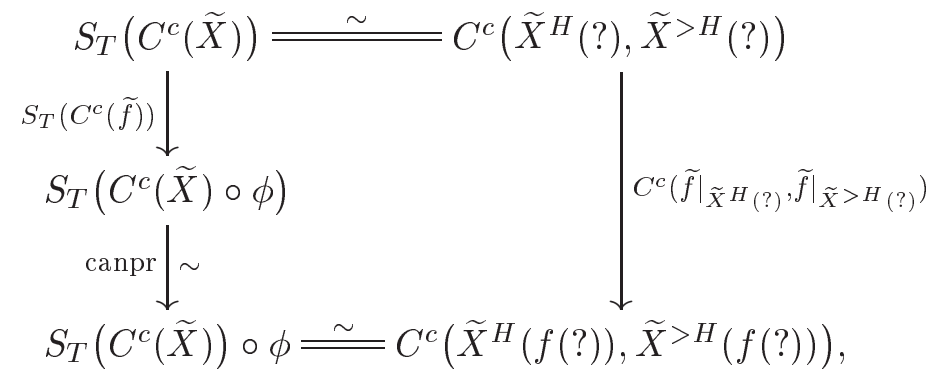

for $? \in T$. So the morphisms $g_{i}$ are

$$
g_{i}=\operatorname{res}_{f^{i}(x)} C^{c}\left(\left.\widetilde{f}\right|_{\widetilde{X}^{H}(?)},\left.\widetilde{f}\right|_{\widetilde{X}>H(?)}\right)=C^{c}\left(\left.\widetilde{f}\right|_{\widetilde{X}^{H}\left(f^{i}(x)\right)},\left.\widetilde{f}\right|_{\widetilde{X}>H\left(f^{i}(x)\right)}\right) .
$$

The morphism $g_{l-1}$ is $g_{l-1}=\operatorname{res}_{\phi_{y, w}}($ canresiso $) \circ \operatorname{res}_{f^{l-1}(x)} S_{T}\left(C^{c}(\widetilde{f})\right)$, and we remember that canresiso was given by $\operatorname{id}_{S_{T}\left(C^{c}(\widetilde{X})\right)} \otimes(-) \circ w^{-1}$ on the $R T$-module $S_{T}\left(C^{c}(\widetilde{X})\right)(?) \otimes_{R T} R T\left(c_{w^{-1}} \operatorname{ind}_{y}(? ?), ?\right)$. So $g_{l-1}$ is precomposition with $w^{-1}$. If $w=(\sigma,[v])$ with $\sigma: G / H \rightarrow G / H, g^{\prime} H \mapsto g^{\prime} g_{w} H$ (here $g_{w} \in N_{G} H$, and $g_{w}$ is only well-defined up to a factor in $H$ ) and $v: G / H \times I \rightarrow X$ with $v_{1}=f^{l}(x)$ and $v_{0}=x \circ \sigma=g_{w} . x$, then $w^{-1}=$ $\left(\sigma^{-1},\left[\left(\sigma^{-1}\right)^{*} v^{-1}\right]\right)$. A point $z$ in $\widetilde{X}^{H}\left(f^{l}(x)\right)$ is identified with a homotopy class of paths $\left[b_{z}\right]$ starting at $z$ and ending at $f^{l}(x)$, and precomposition with $w^{-1}$ is $\left(\mathrm{id},\left[b_{z}\right]\right) \circ\left(\sigma^{-1},\left[\left(\sigma^{-1}\right)^{*} v^{-1}\right]\right)=\left(\sigma^{-1},\left[\left(\sigma^{-1}\right)^{*}\left(b_{z} * w\right)\right]\right)$. This path $\left(\sigma^{-1}\right)^{*}\left(b_{z} * w\right)$ starts at $z \circ \sigma^{-1}=g_{w}^{-1} . z$ and ends at $x$. This is identified with the point $g_{w}^{-1} . z \in \widetilde{X}^{H}(x)$. So geometrically, canresiso is the isomorphism induced by multiplication with $g_{w}^{-1}$,

$$
C^{c}\left(\widetilde{g_{w}^{-1}}\right): C^{c}\left(\widetilde{X}^{H}\left(f^{l}(x)\right), \widetilde{X}^{>H}\left(f^{l}(x)\right)\right) \rightarrow C^{c}\left(\widetilde{X}^{H}(x), \widetilde{X}^{>H}(x)\right) .
$$

This is what we expected: Since there is a path $v$ from $g_{w} \cdot x$ to $f^{l}(x)$, the point $f^{l}(x)$ lies in $g_{w} \cdot X^{H}(x)$, not in $X^{H}(x)$ directly, and letting $g_{w}^{-1}$ act gets us back to $X^{H}(x)$. So the map $g_{l-1}$ is

$$
\left.g_{l-1}=\left.C^{c} \widetilde{\left(g_{w}^{-1} f\right.}\right|_{\widetilde{X}^{H}\left(f^{l-1}(x)\right)},\left.\widetilde{g_{w}^{-1} f}\right|_{\widetilde{X}>H\left(f^{l-1}(x)\right)}\right) .
$$

The splitting result helps us analyze the information contained in the universal functorial Lefschetz invariant.

The $T_{i}^{H}$-parts hold information about fixed points and periodic points. If the length of $T_{i}^{H}$ is $l$, then we know that $X^{H}\left(x_{i}^{H}\right)$ can only contain fixed points of the maps $f^{l}, f^{2 l}, f^{3 l}$ and so on, so if $l \geq 2$ there are no fixed points of $f$ in $X^{H}\left(x_{i}^{H}\right)$. For the study of fixed points pursued in the next chapters, we will therefore restrict ourselves to the case $l=1$, and even more 
restrictively, to the case where $X^{H}(f(x))=X^{H}(x)$. If $l=1$, then it could happen that $f(x) \in g_{w} \cdot X^{H}(x) \neq X^{H}(x)$, this is the case if $g_{w} \in W H$ does not lie in the subgroup $W H_{x}$. Then fixed points cannot appear, but one could have points with $f(x)=g_{w} x$, which implies that $f(G x)=G f(x)$, so the orbit $G x$ would be fixed under $f$, but not pointwise fixed. We will not study these orbits which do not contain fixed points.

The information contained in $T_{i}^{H}$ with $l \geq 2$ comes into play in the study of the Lefschetz zeta function [FH93, FH94, GN93, GN94], which is defined to be

$$
\zeta(f):=\sum_{n \geq 0} \frac{L\left(f^{n}\right)}{n} t^{n} \in \mathbb{Q} \llbracket t \rrbracket .
$$

For example, one immediately reads off that $L\left(f^{n}\right)$ can only be non-zero if there is an $l_{i}^{H}$ which divides $n$. It would be interesting to further explore these connections.

In the cells corresponding to the $Q_{i j}$, no fixed points appear, but the $Q_{i j}$ are nevertheless included in the universal functorial equivariant Lefschetz invariant, they are part of the equivariant Euler characteristic information of the space $X$.

Now let us look at a special case more closely: Suppose that $f(x) \in$ $X^{H}(x)$ and choose a path $w=(\mathrm{id},[v]) \in \operatorname{Mor}(f(x), x)$. Then the map $\left.f\right|_{X^{H}(x)}: X^{H}(x) \rightarrow X^{H}(x)$ is a $W H_{x^{-}}$equivariant endomorphism which induces a $\phi_{x, w}$-twisted endomorphism of the universal covering space $\widetilde{X}^{H}(x)$. Remember that $\operatorname{Aut}(x)$ is a group extension $1 \rightarrow \pi_{1}\left(X^{H}(x), x\right) \rightarrow \operatorname{Aut}(x) \rightarrow$ $W H_{x} \rightarrow 1$, where $W H_{x} \leq W H$ is the subgroup fixing the component $X^{H}(x)$. We are in the situation of Chapter 3. The universal functorial equivariant Lefschetz invariant $\left(U_{W H_{x}}^{\mathbb{Z}}\left(X^{H}(x),\left.f\right|_{X^{H}(x)}\right), u_{W H_{x}}^{\mathbb{Z}}\left(X^{H}(x),\left.f\right|_{X^{H}(x)}\right)\right)$ of $\left.f\right|_{X^{H}(x)}$ is defined, where $U_{W H_{x}}^{\mathbb{Z}}\left(X^{H}(x),\left.f\right|_{X^{H}(x)}\right)=K_{0}\left(\phi-\operatorname{end}_{\mathrm{ffZ} \mathbb{Z} \operatorname{Aut}(x)}\right)$ and $u_{W H_{x}}^{\mathbb{Z}}\left(X^{H}(x),\left.f\right|_{X^{H}(x)}\right)=\left[C^{c}\left(\left.\widetilde{f}\right|_{X^{H}(x)}\right)\right]$. The group $K_{0}\left(\phi-\operatorname{end}_{\mathrm{ffZ} \mathbb{Z} \operatorname{Aut}(x)}\right)$ is the group in which $u_{G}^{\mathbb{Z}}(X, f)_{x}$ takes its value, but note that this value is $u_{G}^{\mathbb{Z}}(X, f)_{x}=\left[C^{c}\left(\left.\widetilde{f}\right|_{X^{H}(x)},\left.\widetilde{f}\right|_{X>H}(x)\right)\right]$.

In general, we easily see that Is $\Pi(G, X)=\coprod_{(H) \in \operatorname{consub} G} \operatorname{Is} \Pi\left(W H, X^{H}\right)$, and this implies that

$$
U_{G}^{R}(X, f) \cong \bigoplus_{(H) \in \operatorname{consub} G} U_{W H}^{R}\left(X^{H},\left.f\right|_{X^{H}}\right)
$$

The element $u_{G}^{R}(X, f)$ maps to the elements given by the relative maps $\left(\left.f\right|_{X^{H}},\left.f\right|_{X>H}\right)$.

Splitting the right hand side into finer parts can become complicated if we are not in the special case considered above. We have to take the union of all the relative spaces $\left(X^{H}\left(f^{i}(x)\right), X^{>H}\left(f^{i}(x)\right)\right)$ with the different subgroups $W H_{f^{i}(x)}$ acting on them, and we have to take into account the elements $g_{w_{i}}$. 
Wrapping up all the considerations of this chapter, we arrive at the slogan: The information given by the equivariant map $f$ on $X$ splits up as a direct sum of the relative information on the fixed point sets.

\subsection{The Illustrative Example, continued}

In Section 3.2, we studied the case where $G=\mathbb{Z} / 2$ acts on $S^{1}$ by reflection at the $x$-axis, and there was a $\mathbb{Z} / 2$-equivariant endomorphism of $S^{1}$ given by $f: S^{1} \rightarrow S^{1}, z \mapsto s^{d}$. What is the meaning of the splitting result for this example?

We need to distinguish two cases. If $d$ is odd, we partition Is $\Pi\left(S^{1}, f\right)$ as $T_{1}=\overline{x_{1}}, T_{2}=\overline{x_{-1}}$ and $T_{3}=\bar{y}$, if $d$ is even, we have $T_{1}=\overline{x_{1}}, Q_{1}=\overline{x_{-1}}$, $T_{2}=\bar{y}$.

So if $d$ is odd,

$$
\begin{aligned}
& K_{0}\left(\phi-\operatorname{end}_{\mathrm{ff} R \Pi\left(S^{1}, f\right)}\right) \\
& \cong K_{0}\left(\phi_{x_{1}, \operatorname{cst}_{x_{1}}}-\operatorname{end}_{\mathrm{ffRAut}\left(x_{1}\right)}\right) \oplus K_{0}\left(\phi_{x_{-1}, \operatorname{cst}_{x_{-}}}-\operatorname{end}_{\mathrm{ffRAut}\left(x_{-1}\right)}\right) \\
& \oplus K_{0}\left(\phi_{y, w} \text {-end } d_{\text {ffR } \operatorname{Aut}(y)}\right) \\
& \cong K_{0}(\operatorname{End}(\text { ff } R)) \oplus K_{0}(\operatorname{End}(\text { ff } R)) \oplus K_{0}\left(d-\operatorname{end}_{\mathrm{ffRAut}(y)}\right) \text {. }
\end{aligned}
$$

If $d$ is even,

$$
\begin{aligned}
& K_{0}\left(\phi-\operatorname{end}_{\mathrm{ff} R \Pi\left(S^{1}, f\right)}\right) \\
& \cong K_{0}\left(\phi_{x_{1}, \operatorname{cst}_{x_{1}}}-\operatorname{end}_{\mathrm{ff} R \operatorname{Aut}\left(x_{1}\right)}\right) \oplus K_{0}\left(\operatorname{ff} R \operatorname{Aut}\left(x_{-1}\right)\right) \\
& \oplus K_{0}\left(\phi_{y, w^{\prime}}-\operatorname{end}_{\mathrm{ff} R \operatorname{Aut}(y)}\right) \\
& \cong K_{0}(\operatorname{End}(\operatorname{ff} R)) \oplus K_{0}(\mathrm{ff} R) \oplus K_{0}\left(d-\operatorname{end}_{\mathrm{ffRAut}(y)}\right) .
\end{aligned}
$$

We see that $K_{0}\left(\phi\right.$-end $\left.\mathrm{ff}_{\mathrm{f} \Pi\left(S^{1}, f\right)}\right)$ splits up as

$$
\begin{aligned}
& K_{0}\left(\phi-\operatorname{end}_{\mathrm{ff} R \Pi\left(S^{1}, f\right)}\right) \\
& \cong \begin{cases}K_{0}(\operatorname{End}(\mathrm{ff} R)) \oplus K_{0}(\operatorname{End}(\mathrm{ff} R)) \oplus K_{0}\left(d-\operatorname{end}_{\mathrm{ff} R \operatorname{Aut}(y)}\right) & \text { if } d \text { is odd } \\
K_{0}(\operatorname{End}(\mathrm{ff} R)) \oplus K_{0}(\mathrm{ff} R) \oplus K_{0}\left(d-\operatorname{end}_{\mathrm{ff} R \operatorname{Aut}(y)}\right) & \text { if } d \text { is even. }\end{cases}
\end{aligned}
$$

The element $u_{\mathbb{Z} / 2}^{R}\left(S^{1}, f\right)$ is given by

$$
u_{\mathbb{Z} / 2}^{R}\left(S^{1}, f\right)= \begin{cases}{\left[\operatorname{id}_{R}\right] \oplus\left[\operatorname{id}_{R}\right] \oplus\left([(\cdot d)] \oplus\left[\left(\cdot d+\frac{d-1}{2}\right)\right]-\left[d^{\prime}\right]\right)} & \text { if } d \text { is odd } \\ {\left[\operatorname{id}_{R}\right] \oplus[R] \oplus\left([(\cdot d)] \oplus[0] \oplus-\left[d^{\prime \prime}\right]\right)} & \text { if } d \text { is even }\end{cases}
$$

where $d^{\prime}$ and $d^{\prime \prime}$ are defined in Section 3.2. 


\section{Chapter 7}

\section{The Generalized Equivariant Lefschetz Invariant}

In this chapter we develop a generalized equivariant Lefschetz invariant $\left(\Lambda_{G}(X, f), \lambda_{G}(f)\right)$. It is an equivariant analog of the generalized Lefschetz invariant [Rei38, Wec41] and a refinement of the equivariant Lefschetz class [LR03, Definition 3.6]. A trace map $\operatorname{tr}_{G(X, f)}$ which maps the universal functorial equivariant Lefschetz invariant $\left(U_{G}^{\mathbb{Z}}(X, f), u_{G}^{\mathbb{Z}}(X, f)\right)$ to the generalized equivariant Lefschetz invariant $\left(\Lambda_{G}(X, f), \lambda_{G}(f)\right)$ is constructed.

We start by defining the group $\Lambda_{G}(X, f)$ in which the generalized equivariant Lefschetz invariant takes its values. We are looking for an invariant giving fixed point information. So we are only interested in information lying in objects $(x: G / H \rightarrow X) \in \Pi(G, X)$ with $X^{H}(f(x))=X^{H}(x)$. That means there is a morphism $w_{x}=\left(\mathrm{id},\left[v_{x}\right]\right) \in \operatorname{Mor}(f(x), x)$. Concentrating on $x \in \Pi(G, X)$ with $X^{H}(f(x))=X^{H}(x)$, the splitting of the target group $U_{G}^{\mathbb{Z}}(X, f)$ of the universal functorial equivariant Lefschetz invariant $u_{G}^{\mathbb{Z}}(X, f)$ is

$$
U_{G}^{\mathbb{Z}}(X, f) \cong \bigoplus_{\substack{\bar{x} \in \operatorname{Is} \Pi(G, X), X^{H}(f(x))=X^{H}(x)}} U_{G}^{\mathbb{Z}}(X, f)_{\bar{x}} \oplus \text { other terms, }
$$

where for $\bar{x} \in \operatorname{Is}(G, X)$ with $X^{H}(f(x))=X^{H}(x)$ we have

$$
U_{G}^{\mathbb{Z}}(X, f)_{\bar{x}}:=\coprod_{\substack{x \in \bar{x}, w_{x} \in \operatorname{Mor}(f(x), x)}} K_{0}\left(\phi_{x, w_{x}}-\operatorname{end}_{\mathrm{ffZ}} \operatorname{Aut}(x)\right) / \sim .
$$

The target group $\Lambda_{G}(X, f)$ is designed in the same way. For objects $x \in$ $\operatorname{Ob} \Pi(G, X)$ with $X^{H}(f(x))=X^{H}(x)$, we can always find morphisms $w_{x}=$ (id, $\left.\left[v_{x}\right]\right) \in \operatorname{Mor}(f(x), x)$, and we restrict our attention to morphisms of that form. We define a suitable group $\mathbb{Z} \pi_{1}\left(X^{H}(x), x\right)_{\phi_{x, w_{x}}^{\prime}}$. Then we specify the isomorphism arising when changing the morphism $w_{x}$ and the base point $x$, therefore arriving at a definition independent of the choices of $x$ and $w_{x}$. 
In the next step, for all $x \in \mathrm{Ob} \Pi(G, X)$ with $X^{H}(f(x))=X^{H}(x)$ and morphisms $w_{x}=\left(\mathrm{id},\left[v_{x}\right]\right) \in \operatorname{Mor}(f(x), x)$, we define a trace map which is defined on $K_{0}\left(\phi_{x, w_{x}}-\operatorname{end}_{\mathrm{ff} \mathbb{Z} \operatorname{Aut}(x)}\right)$ and lands in the group $\mathbb{Z} \pi_{1}\left(X^{H}(x), x\right)_{\phi_{x, w_{x}}^{\prime}}$. We know that $\operatorname{Aut}(x)$ is a group extension

$$
1 \rightarrow \pi_{1}\left(X^{H}(x), x\right) \rightarrow \operatorname{Aut}(x) \rightarrow W H_{x} \rightarrow 1
$$

We have a commutative diagram

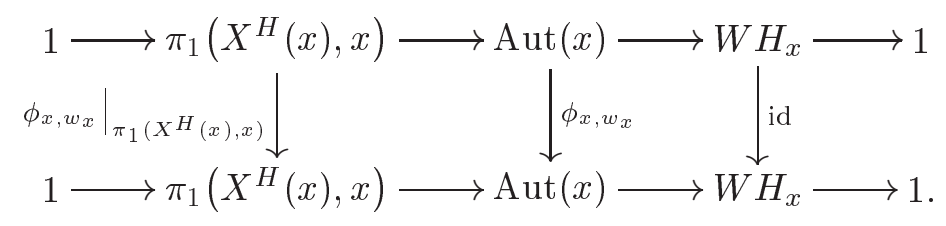

The endomorphism $\phi_{x, w_{x}}$ is induced by the endomorphism $\left.\phi_{x, w_{x}}\right|_{\pi_{1}\left(X^{H}(x), x\right)}$ on the $\pi_{1}\left(X^{H}(x), x\right)$-part, the map $\phi_{x, w_{x}}$ becomes trivial once we divide out the normal subgroup $\pi_{1}\left(X^{H}(x), x\right)$ of $\operatorname{Aut}(x)$. This is the clue to what we have in mind for the definition of the trace map $\operatorname{tr}_{G(X, f)}$ : We combine the trace maps $\operatorname{tr}_{R G}: R G \rightarrow R, \sum_{g \in G} r_{g} \cdot g \mapsto r_{1}$ [LR03, Equations 1.1 and 1.2], applying $\operatorname{tr}_{R G}$ to the $W H_{x}$-part, and $\operatorname{tr}_{(\mathbb{Z} \pi, \phi)}: \mathbb{Z} \pi \rightarrow \mathbb{Z} \pi_{\phi}, \sum_{\gamma \in \pi} n_{\gamma} \cdot \gamma \mapsto$ $\sum_{\gamma \in \pi} n_{\gamma} \cdot \bar{\gamma}$ [Lüc99, 3.6], applying $\operatorname{tr}_{(\mathbb{Z} \pi, \phi)}$ to the $\pi_{1}\left(X^{H}(x), x\right)$-part. For simplicity of notation, we write $\phi$ instead of $\phi_{x, w_{x}}$ in the sequel if no confusion can occur.

\subsection{The Target Group}

The group $\mathbb{Z} \pi_{1}\left(X^{H}(x), x\right)_{\phi_{x, w_{x}}^{\prime}}$ is defined in analogy to $\mathbb{Z} \pi_{1}\left(X^{H}(x), x\right)_{\phi_{x, w_{x}}}$ [Lüc99, 3.6]. We repeat the definition of $\mathbb{Z} \pi_{1}\left(X^{H}(x), x\right)_{\phi_{x, w_{x}}}$ for convenience.

Definition 7.1.1. Let $\phi_{x, w_{x}}: \pi_{1}\left(X^{H}(x), x\right) \rightarrow \pi_{1}\left(X^{H}(x), x\right)$ be a group endomorphism. We define

$$
\mathbb{Z} \pi_{1}\left(X^{H}(x), x\right)_{\phi_{x, w_{x}}}:=\mathbb{Z} \pi_{1}\left(X^{H}(x), x\right) / \phi_{x, w_{x}}(\gamma) \alpha \gamma^{-1} \sim \alpha,
$$

where $\alpha, \gamma \in \pi_{1}\left(X^{H}(x), x\right)$.

Generalizing, we obtain the following definition.

Definition 7.1.2. For $x \in \operatorname{Ob} \Pi(G, X)$ with $X^{H}(f(x))=X^{H}(x)$ and a morphism $w_{x}=\left(\mathrm{id},\left[v_{x}\right]\right) \in \operatorname{Mor}(f(x), x)$, set

$$
\mathbb{Z} \pi_{1}\left(X^{H}(x), x\right)_{\phi_{x, w_{x}}^{\prime}}:=\mathbb{Z} \pi_{1}\left(X^{H}(x), x\right) / \phi_{x, w_{x}}(\gamma) \alpha \gamma^{-1} \sim \alpha,
$$

where $\alpha \in \pi_{1}\left(X^{H}(x), x\right), \gamma \in \operatorname{Aut}(x)$ and $\phi_{x, w_{x}}(\gamma)=w_{x} \phi(\gamma) w_{x}^{-1} \in \operatorname{Aut}(x)$. 
Note that $\mathbb{Z} \pi_{1}\left(X^{H}(x), x\right)_{\phi_{x, w_{x}}^{\prime}}$ is an abelian group with respect to addition. We identify the group $\pi_{1}\left(X^{H}(x), x\right)$ with the subgroup $\{(\mathrm{id},[v]) \mid[v] \in$ $\left.\pi_{1}\left(X^{H}(x), x\right)\right\} \leq \operatorname{Aut}(x)$. We have $\phi_{x, w_{x}}(\gamma) \alpha \gamma^{-1} \in \pi_{1}\left(X^{H}(x), x\right)$ for all $\gamma \in \operatorname{Aut}(x)$ and $\alpha \in \pi_{1}\left(X^{H}(x), x\right)$ because the map $\phi_{x, w_{x}}$ does not change the $W H_{x}$-part of the morphism $\gamma$ and $\pi_{1}\left(X^{H}(x), x\right)$ is normal: If $\gamma=$ $\left(\sigma_{\gamma},\left[v_{\gamma}\right]\right) \in \operatorname{Aut}(x)$ and $\alpha=(\operatorname{id},[\alpha]) \in \operatorname{Aut}(x)$, then $\phi_{x, w_{x}}(\gamma) \alpha \gamma^{-1}=$ $\left(\sigma_{\gamma} \mathrm{id} \sigma_{\gamma}^{-1},\left[v_{\phi_{x, w_{x}}(\gamma)} \alpha v_{\gamma^{-1}}\right]\right)=\left(\mathrm{id},\left[\sigma_{\gamma}^{*}\left(v_{x}\right) f\left(v_{\gamma}\right) v_{x}^{-1} \alpha v_{\gamma^{-1}}\right]\right) \in \pi_{1}\left(X^{H}(x), x\right)$. This is important, otherwise the definition would not make much sense.

The group $\mathbb{Z} \pi_{1}\left(X^{H}(x), x\right)_{\phi_{x, w_{x}}^{\prime}}$ is not the same as $\mathbb{Z} \pi_{1}\left(X^{H}(x), x\right)_{\phi_{x, w_{x}}}$. We divide out more, we allow $\gamma \in \operatorname{Aut}(x)$, not only $\gamma \in \pi_{1}\left(X^{H}(x), x\right)$. It is a trivial fact that there is a projection map

$$
\mathbb{Z} \pi_{1}\left(X^{H}(x), x\right)_{\phi_{x, w_{x}}} \rightarrow \mathbb{Z} \pi_{1}\left(X^{H}(x), x\right)_{\phi_{x, w_{x}}^{\prime}} .
$$

Remark 7.1.3. We have to use the group $\mathbb{Z} \pi_{1}\left(X^{H}(x), x\right)_{\phi_{x, w_{x}}^{\prime}}$ instead of the group $\mathbb{Z} \pi_{1}\left(X^{H}(x), x\right)_{\phi_{x, w_{x}}}$ because we want the definition of the trace map to be independent of the choices we make, and this can only be ensured by dividing out more elements. More precisely, this is needed for example in the proof of the first assertion of Lemma 7.2.2, the verification of the trace property.

What happens if we change the morphism $w_{x}$ ? Given another morphism $w_{x}^{\prime}=\left(\mathrm{id},\left[v_{x}^{\prime}\right]\right) \in \operatorname{Mor}(f(x), x)$, there is a group isomorphism

$$
\begin{aligned}
\psi_{w_{x}^{\prime}, w_{x}}: \mathbb{Z} \pi_{1}\left(X^{H}(x), x\right)_{\phi_{x, w_{x}}^{\prime}} & \stackrel{\sim}{\rightarrow} \mathbb{Z} \pi_{1}\left(X^{H}(x), x\right)_{\phi_{x, w_{x}^{\prime}}^{\prime}} \\
\alpha & \mapsto v_{x}^{\prime} v_{x}^{-1} \alpha .
\end{aligned}
$$

We can write either $v$ or $w$ since $v$ is identified with $w=(\mathrm{id},[v])$ under the inclusion $\pi_{1}\left(X^{H}(x), x\right) \rightarrow \operatorname{Aut}(x)$. The isomorphism $\psi_{w_{x}^{\prime}, w_{x}}$ respects the equivalence relation: We have

$$
\phi_{x, w_{x}}(\gamma) \alpha \gamma^{-1} \mapsto v_{x}^{\prime} v_{x}^{-1} v_{x} \phi(\gamma) v_{x}^{-1} \alpha \gamma^{-1}=\phi_{x, w_{x}^{\prime}}(\gamma) v_{x}^{\prime} v_{x}^{-1} \alpha \gamma^{-1} \sim v_{x}^{\prime} v_{x}^{-1} \alpha .
$$

So the map $\psi_{w_{x}^{\prime}, w_{x}}$ is well-defined. It is a homomorphism of abelian groups with respect to addition. The inverse map is given by $\beta \mapsto v_{x}\left(v_{x}^{\prime}\right)^{-1} \beta$. As usual, we construct a group which does not depend on the choice of the morphism.

Definition 7.1.4. We set

$$
\mathbb{Z} \pi_{1}\left(X^{H}(x), x\right)_{\phi_{x}^{\prime}}:=\coprod_{\substack{w_{x}=\left(\mathrm{id},\left[v_{x}\right]\right) \in \\ \operatorname{Mor}(f(x), x)}} \mathbb{Z} \pi_{1}\left(X^{H}(x), x\right)_{\phi_{x, w_{x}}^{\prime}} / \psi_{w_{x}, w_{x}^{\prime}}(\alpha) \sim \alpha .
$$

The obvious inclusion induces an isomorphism

$$
\psi_{w_{x}}: \mathbb{Z} \pi_{1}\left(X^{H}(x), x\right)_{\phi_{x, w_{x}}^{\prime}} \stackrel{\sim}{\rightarrow} \mathbb{Z} \pi_{1}\left(X^{H}(x), x\right)_{\phi_{x}^{\prime}} .
$$


In the end, we want a definition which does not depend on the basepoint $x \in$ $\operatorname{Ob}(\Pi(G, X))$, so it is worth thinking about how the group changes when we change the basepoint: For a morphism $(\sigma,[t]) \in \operatorname{Mor}_{\Pi(G, X)}(x(H), y(K))$, where $X^{H}(f(x))=X^{H}(x)$ and $X^{K}(f(y))=X^{K}(y)$, we choose morphisms $w_{x}=\left(\mathrm{id},\left[v_{x}\right]\right) \in \operatorname{Mor}(f(x), x)$ and $w_{y}=\left(\mathrm{id},\left[v_{y}\right]\right) \in \operatorname{Mor}(f(y), y)$ and set

$$
\begin{aligned}
(\sigma,[t])_{w_{x}, w_{y}}^{*}: \mathbb{Z} \pi_{1}\left(X^{K}(y), y\right)_{\phi_{y, w_{y}}^{\prime}} & \rightarrow \mathbb{Z} \pi_{1}\left(X^{H}(x), x\right)_{\phi_{x, w_{x}}^{\prime}} \\
\alpha & \mapsto v_{x} f\left(t^{-1}\right) \sigma^{*}\left(v_{y}^{-1}\right) \sigma^{*}(\alpha) t .
\end{aligned}
$$

This is well-defined: For $\gamma \in \operatorname{Aut}(y)$ and $\alpha \in \mathbb{Z} \pi_{1}\left(X^{K}(y), y\right)$, we have

$$
\begin{aligned}
& \phi_{y, w_{y}}(\gamma) \alpha \gamma^{-1}=v_{y} \phi(\gamma) v_{y}^{-1} \alpha \gamma^{-1} \\
& \mapsto \quad v_{x} f\left(t^{-1}\right) \sigma^{*}\left(v_{y}^{-1}\right) \sigma^{*}\left(v_{y} \phi(\gamma) v_{y}^{-1} \alpha \gamma^{-1}\right) t \\
&=v_{x} f\left(t^{-1}\right) \sigma^{*}(\phi(\gamma)) \sigma^{*}\left(v_{y}^{-1}\right) \sigma^{*}(\alpha) t t^{-1} \sigma^{*}\left(\gamma^{-1}\right) t \\
&=v_{x} f(\widetilde{\gamma}) f\left(t^{-1}\right) \sigma^{*}\left(v_{y}^{-1}\right) \sigma^{*}(\alpha) t \widetilde{\gamma}^{-1} \\
&=v_{x} \phi(\widetilde{\gamma}) v_{x}^{-1} v_{x} f\left(t^{-1}\right) \sigma^{*}\left(v_{y}^{-1}\right) \sigma^{*}(\alpha) t \widetilde{\gamma}^{-1} \\
& \sim v_{x} f\left(t^{-1}\right) \sigma^{*}\left(v_{y}^{-1}\right) \sigma^{*}(\alpha) t \\
&=(\sigma,[t])_{w_{x}, w_{y}}(\alpha),
\end{aligned}
$$

with $\widetilde{\gamma}:=t^{-1} \sigma^{*}(\gamma) t \in \operatorname{Aut}(x)$.

If $(\sigma,[t]) \in \operatorname{Mor}_{\Pi(G, X)}(x(H), y(K))$ and $(\tau,[s]) \in \operatorname{Mor}_{\Pi(G, X)}(y(K), z(L))$, where $X^{H}(f(x))=X^{H}(x), X^{K}(f(y))=X^{K}(y)$ and $X^{L}(f(z))=X^{L}(z)$, for $\alpha \in \mathbb{Z} \pi_{1}\left(X^{L}(z)\right)_{\phi_{z, w_{z}}^{\prime}}$ we have

$$
\begin{aligned}
& (\sigma,[t])_{w_{x}, w_{y}}^{*}(\tau,[s])_{w_{y}, w_{z}}^{*}(\alpha) \\
& \quad=v_{x} f\left(t^{-1}\right) \sigma^{*}\left(v_{y}^{-1}\right) \sigma^{*}\left(v_{y} f\left(s^{-1}\right) \tau^{*}\left(v_{z}^{-1}\right) \tau^{*}(\alpha) s\right) t \\
& \quad=v_{x} f\left(t^{-1} \sigma^{*}\left(s^{-1}\right)\right)(\tau \sigma)^{*}\left(v_{z}^{-1}\right)(\tau \sigma)^{*}(\alpha) \sigma^{*}(s) t \\
& \quad=\left(\tau \sigma,\left[\sigma^{*}(s) t\right]\right)_{w_{x}, w_{z}}^{*}(\alpha) \\
& \quad=((\tau,[s])(\sigma,[t]))_{w_{x}, w_{z}}^{*}(\alpha) .
\end{aligned}
$$

The above map reduces to the following map if we choose $w_{x}=\left(\mathrm{id},\left[v_{x}\right]\right)$ with $v_{x}=t^{-1} \sigma^{*}\left(v_{y}\right) f(t)$ :

$$
\begin{aligned}
(\sigma,[t])_{w_{x}, w_{y}}^{*}: \mathbb{Z} \pi_{1}\left(X^{K}(y), y\right)_{\phi_{y, w_{y}}^{\prime}} & \rightarrow \mathbb{Z} \pi_{1}\left(X^{H}(x), x\right)_{\phi_{x, w_{x}}^{\prime}} \\
\alpha & \mapsto t^{-1} \sigma^{*}\left(v_{y}\right) f(t) f\left(t^{-1}\right) \sigma^{*}\left(v_{y}^{-1}\right) \sigma^{*}(\alpha) t \\
& =t^{-1} \sigma^{*}(\alpha) t .
\end{aligned}
$$

If $(\sigma,[t])$ is an isomorphism, we have $(\sigma, t)^{-1}(\mathrm{id}, \alpha)(\sigma, t)=\left(\mathrm{id}, t^{-1} \sigma^{*}(\alpha) t\right)$, so in this case $(\sigma,[t])_{w_{x}, w_{y}}^{*}$ for $v_{x}=t^{-1} \sigma^{*}\left(v_{y}\right) f(t)$ is just conjugation with $(\sigma,[t])$. We obtain as a special case for $(\mathrm{id},[t]) \in \operatorname{Aut}(x)$ the map $\psi_{w_{x}^{\prime}, w_{x}}$ :

$$
\begin{aligned}
(\mathrm{id},[t])_{w_{x}^{\prime}, w_{x}}^{*}: \mathbb{Z} \pi_{1}\left(X^{H}(x), x\right)_{\phi_{y, w_{x}}^{\prime}} & \rightarrow \mathbb{Z} \pi_{1}\left(X^{H}(x), x\right)_{\phi_{x, w_{x}^{\prime}}^{\prime}} \\
\alpha & \mapsto v_{x}^{\prime} f\left(t^{-1}\right) v_{x}^{-1} \alpha t=v_{x}^{\prime} v_{x}^{-1} \alpha .
\end{aligned}
$$


It is very interesting that the map $(\sigma,[t])_{w_{x}, w_{y}}^{*}$ does not depend on the choice of $(\sigma,[t]) \in \operatorname{Mor}(x, y)$.

Lemma 7.1.5. For $\bar{\alpha} \in \pi_{1}\left(X^{K}(y), y\right)_{\phi_{y, w y}^{\prime}}$ we have

$$
(\sigma,[t])_{w_{x}, w_{y}}^{*}(\bar{\alpha})=(\tau,[s])_{w_{x}, w_{y}}^{*}(\bar{\alpha}) \in \mathbb{Z} \pi_{1}\left(X^{H}(x), x\right)_{\phi_{x, w_{x}}^{\prime}}
$$

for all morphisms $(\sigma,[t]),(\tau,[s]) \in \operatorname{Mor}(x, y)$.

Proof. For all $\gamma=\left(\sigma_{\gamma},\left[v_{\gamma}\right]\right) \in \operatorname{Aut}(x)$, we have

$$
\begin{aligned}
(\sigma,[t])_{w_{y}, w_{x}}^{*}(\bar{\alpha}) & =\overline{v_{x} f(\gamma) v_{x}^{-1}(\sigma,[t])_{w_{x}, w_{y}}^{*}(\alpha) \gamma^{-1}} \\
& =\overline{v_{x} \sigma_{\gamma}^{-1^{*}} f\left(v_{\gamma} t^{-1}\right) \sigma_{\gamma}^{-1^{*}} \sigma^{*}\left(v_{y}^{-1}\right) \sigma_{\gamma}^{-1^{*}} \sigma^{*}(\alpha) \sigma_{\gamma}^{-1^{*}}\left(t v_{\gamma}^{-1}\right)} .
\end{aligned}
$$

Setting $\sigma_{\gamma}=\tau^{-1} \sigma$ and $v_{\gamma}=\left(\tau^{-1} \sigma\right)^{*}\left(s^{-1}\right) t$, we obtain

$$
\begin{aligned}
(\sigma,[t])_{w_{y}, w_{x}}^{*}(\bar{\alpha}) & =\overline{v_{x} f\left(s^{-1}\right) \tau^{*}\left(v_{y}^{-1}\right) \tau^{*}(\alpha) s} \\
& =(\tau,[s])_{w_{x}, w_{y}}^{*}(\bar{\alpha}) .
\end{aligned}
$$

We can formulate $(\sigma,[t])^{*}$ in terms of the groups $\mathbb{Z} \pi_{1}\left(X^{H}(x), x\right)_{\phi_{x}^{\prime}}$, independently of the morphisms $w_{x}$ and $w_{y}$.

Definition 7.1.6. Let $(\sigma,[t]) \in \operatorname{Mor}(x, y)$. We define

$$
(\sigma,[t])^{*}:=\psi_{w_{x}}(\sigma,[t])_{w_{x}, w_{y}}^{*} \psi_{w_{y}}^{-1}: \mathbb{Z} \pi_{1}\left(X^{K}(y), y\right)_{\phi_{y}^{\prime}} \rightarrow \mathbb{Z} \pi_{1}\left(X^{H}(x), x\right)_{\phi_{x}^{\prime}}
$$

for any choice of morphisms $w_{x}=\left(\mathrm{id},\left[v_{x}\right]\right) \in \operatorname{Mor}(f(x), x)$ and $w_{y}=$ (id, $\left.\left[v_{y}\right]\right) \in \operatorname{Mor}(f(y), y)$.

This definition does not depend on the choice of $w_{x}$ and $w_{y}$ since for any other choices $w_{x}^{\prime}$ and $w_{y}^{\prime}$ we have

$$
\left(\psi_{w_{x}^{\prime}, w_{x}}\right)^{-1}(\sigma,[t])_{w_{x}^{\prime}, w_{y}^{\prime}}^{*}\left(\psi_{w_{y}^{\prime}, w_{y}}\right)=(\sigma,[t])_{w_{x}, w_{y}}^{*}
$$

by the following calculation: For $\alpha \in \mathbb{Z} \pi_{1}\left(X^{K}(y), y\right)_{\phi_{y, w_{y}}^{\prime}}$ we have

$$
\begin{aligned}
& \left(\left(\psi_{w_{x}^{\prime}, w_{x}}\right)^{-1}(\sigma,[t])_{w_{x}^{\prime}, w_{y}^{\prime}}^{*}\left(\psi_{w_{y}^{\prime}, w_{y}}\right)\right)(\alpha) \\
& \quad=v_{x}\left(v_{x}^{\prime}\right)^{-1} v_{x}^{\prime} f\left(t^{-1}\right) \sigma^{*}\left(v_{y}^{\prime}\right)^{-1} \sigma^{*}\left(v_{y}^{\prime} v_{y}^{-1} \alpha\right) t \\
& \quad=(\sigma,[t])_{w_{x}, w_{y}}^{*}(\alpha) .
\end{aligned}
$$

In particular, for $(\mathrm{id},[t]) \in \operatorname{Aut}(x)$ we have $(\mathrm{id},[t])^{*}=\mathrm{id}_{\mathbb{Z} \pi_{1}\left(X^{H}(x), x\right)_{\phi_{x}^{\prime}}}$.

We have defined an abelian group $\mathbb{Z} \pi_{1}\left(X^{H}(x), x\right)_{\phi_{x}^{\prime}}$ for all $x \in \Pi(G, X)$ with $X^{H}(f(x))=X^{H}(x)$ and have shown that the application mapping $x$ to $\mathbb{Z} \pi_{1}\left(X^{H}(x), x\right)_{\phi_{x}^{\prime}}$ has the properties necessary for a contravariant functor from $\Pi(G, X)$ to $\mathcal{A} b$. We extend the application by 0 to all objects of $\Pi(G, X)$ and make the following definition. 
Definition 7.1.7. We define the contravariant functor $\mathbb{Z} \Pi(G, X)_{\phi}$ to be

$$
\begin{aligned}
\mathbb{Z} \Pi(G, X)_{\phi}: \Pi(G, X) & \rightarrow \mathcal{A} b \\
x & \mapsto \begin{cases}\mathbb{Z} \pi_{1}\left(X^{H}(x), x\right)_{\phi_{x}^{\prime}} & \text { if } X^{H}(f(x))=X^{H}(x) \\
0 & \text { else, }\end{cases} \\
(\sigma,[t]) & \mapsto \begin{cases}(\sigma,[t])^{*} & \text { if } X^{K}(f(y))=X^{K}(y) \\
0 & \text { else. }\end{cases}
\end{aligned}
$$

Here $\mathbb{Z} \pi_{1}\left(X^{H}(x), x\right)_{\phi_{x}^{\prime}}$ is defined in Definition 7.1 .4 and $(\sigma,[t])^{*}$ is defined in Definition 7.1.6.

If there is a morphism $(\sigma,[t])$ from $x$ to $y$, then $X^{K}(f(y))=X^{K}(y)$ implies that $X^{H}(f(x))=X^{H}(x)$, so this is well-defined. We set

$$
\mathbb{Z} \Pi(G, X)_{\phi, \bar{x}}:=\coprod_{x \in \bar{x}} \mathbb{Z} \Pi(G, X)_{\phi, x} / \sim,
$$

where $(\sigma,[t])^{*}(\alpha) \sim \alpha$ for all $(\sigma,[t]): x \stackrel{\sim}{\rightarrow} x^{\prime}$. The obvious inclusion map induces for $x$ with $X^{H}(f(x))=X^{H}(x)$ an isomorphism

$$
\psi_{x}: \mathbb{Z} \pi_{1}\left(X^{H}(x), x\right)_{\phi_{x}^{\prime}} \stackrel{\sim}{\rightarrow} \mathbb{Z} \Pi(G, X)_{\phi, \bar{x}} .
$$

Now we can define the group in which the generalized equivariant Lefschetz invariant takes its values.

Definition 7.1.8. We define

$$
\Lambda_{G}(X, f):=\bigoplus_{\substack{\bar{x} \in \operatorname{Is} \Pi(G, X), X^{H}(f(x))=X^{H}(x)}} \mathbb{Z} \Pi(G, X)_{\phi, \bar{x}} \oplus \bigoplus_{\begin{array}{c}
\bar{x} \in \operatorname{Is} \Pi(G, X), \\
X^{H}(f(x)) \neq X^{H}(x)
\end{array}} 0 .
$$

\subsection{A Convenient Trace Map}

In this section, we develop the trace map applicable to our situation. For all $x \in \mathrm{Ob} \Pi(G, X)$ with $X^{H}(f(x))=X^{H}(x)$ and morphisms $w_{x}=\left(\mathrm{id},\left[v_{x}\right]\right) \in$ $\operatorname{Mor}(f(x), x)$, we construct a trace map $\operatorname{tr}_{R} \operatorname{Aut}(x)$ defined on the abelian group $K_{0}\left(\phi_{x, w_{x}}-\operatorname{end}_{\mathrm{fp} \mathbb{Z} \operatorname{Aut}(x)}\right)$ and taking values in $\mathbb{Z} \pi_{1}\left(X^{H}(x), x\right)_{\phi_{x, w_{x}}^{\prime}}$. Here fp stands for finitely generated projective modules, these appear in Chapter 8. We combine the trace maps $\operatorname{tr}_{R G}: R G \rightarrow R, \sum_{g \in G} r_{g} \cdot g \mapsto r_{1}$ [LR03, Equations 1.1 and 1.2], applying this map $\operatorname{tr}_{R G}$ to the $W H_{x}$-part, and $\operatorname{tr}_{(\mathbb{Z} \pi, \phi)}: \mathbb{Z} \pi \rightarrow \mathbb{Z} \pi_{\phi}, \sum_{\gamma \in \pi} n_{\gamma} \cdot \gamma \mapsto \sum_{\gamma \in \pi} n_{\gamma} \cdot \bar{\gamma}$ [Lüc99, 3.6], applying this map $\operatorname{tr}_{(\mathbb{Z} \pi, \phi)}$ to the $\pi_{1}\left(X^{H}(x), x\right)$-part. We start by defining a map

$$
\operatorname{tr}_{\mathbb{Z} \operatorname{Aut}(x)}: \operatorname{Ob}\left(\phi_{x, w_{x}}-\operatorname{end}_{\mathrm{fp} \mathbb{Z} A u t(x)}\right) \rightarrow \mathbb{Z} \pi_{1}\left(X^{H}(x), x\right)_{\phi_{x, w_{x}}^{\prime}} .
$$


We obtain the map $\operatorname{tr}_{\mathbb{Z} \operatorname{Aut}(x)}$ in analogy to Lück and Rosenberg [LR03, Chapter 1] by defining a map

$$
\operatorname{tr}_{\mathbb{Z} \operatorname{Aut}(x)}: \mathbb{Z} \operatorname{Aut}(x) \rightarrow \mathbb{Z} \pi_{1}\left(X^{H}(x), x\right)_{\phi_{x, w_{x}}^{\prime}}
$$

and checking that its extension to matrices over $\mathbb{Z} \operatorname{Aut}(x)$ induces a welldefined map. We formulate the definition of the trace map $\operatorname{tr}_{R G}$ independently of the concrete group Aut $(x)$ which is given to us geometrically.

Definition 7.2.1. Let $\pi$ and $W$ be discrete groups, and let $G$ be a group extension $1 \rightarrow \pi \rightarrow G \rightarrow W \rightarrow 1$. Let an endomorphism $\phi: G \rightarrow G$ be given that restricts to an endomorphism $\phi_{\pi}: \pi \rightarrow \pi$ and becomes trivial when the normal subgroup $\pi \leq G$ is divided out, i.e., lying in a commutative diagram

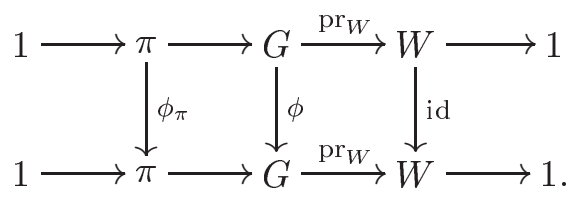

Let $R$ be a commutative associative ring with unit. Let

$$
R \pi_{\phi^{\prime}}:=R \pi / \sim,
$$

where $\phi(\gamma) \alpha \gamma \sim \alpha$ for $\alpha \in \pi$ and $\gamma \in G$. Note that $\alpha \in \pi$ implies that $\phi(\gamma) \alpha \gamma^{-1} \in \pi$ since

$$
\operatorname{pr}_{W}\left(\phi(\gamma) \alpha \gamma^{-1}\right)=\operatorname{id}_{W}\left(\operatorname{pr}_{W}(\gamma)\right) \operatorname{pr}_{W}(\alpha) \operatorname{pr}_{W}\left(\gamma^{-1}\right)=1_{W}
$$

Let $\phi$-end $\mathrm{f}_{\mathrm{f} R G}$ denote the category of $\phi$-twisted endomorphisms of finitely generated projective $R G$-modules. We define the trace map

$$
\operatorname{tr}_{R G}: \mathrm{Ob}\left(\phi-\mathrm{end}_{\mathrm{fp} R G}\right) \rightarrow R \pi_{\phi^{\prime}}
$$

as follows: On $R G$, we set

$$
\begin{aligned}
\operatorname{tr}_{R G}: R G & \rightarrow R \pi_{\phi^{\prime}} \\
\sum_{g \in G} r_{g} \cdot g & \mapsto \sum_{g \in \pi} r_{g} \cdot \bar{g},
\end{aligned}
$$

where $-: \pi \rightarrow \pi_{\phi^{\prime}}, g \mapsto \bar{g}$ denotes the projection. Given a $\phi$-twisted endomorphism $u: P \rightarrow \operatorname{res}_{\phi} P$ of a finitely generated projective $R G$-module, we choose a finitely generated projective $R G$-module $Q$ and an isomorphism $v: P \oplus Q \stackrel{\sim}{\rightarrow} \bigoplus_{i \in I} R G$ for a finite indexing set $I$. Then we have a $\phi$-twisted endomorphism

$$
\phi^{*}(v) \circ(u \oplus 0) \circ v^{-1}: \bigoplus_{i \in I} R G \rightarrow \operatorname{res}_{\phi}\left(\bigoplus_{i \in I} R G\right),
$$

to which a matrix $A=\left(a_{i j}\right)_{i, j \in I}$ is associated. We define

$$
\operatorname{tr}_{R G}(u):=\sum_{i \in I} \operatorname{tr}_{R G}\left(a_{i i}\right) \in R \pi_{\phi^{\prime}}
$$


As usual, the definition is independent of the choices involved. The trace map $\operatorname{tr}_{R G}$ has the following properties corresponding to the trace map $\operatorname{tr}_{R G}$ of Lück and Rosenberg [LR03, Lemma 1.3]:

Lemma 7.2.2. Let $G$ be a discrete group extension with endomorphism as in Definition 7.2.1, i.e., a group extension $1 \rightarrow \pi \rightarrow G \rightarrow W \rightarrow 1$ with endomorphism $\phi: G \rightarrow G$ which restricts to $\pi$ and such that $\phi_{W}=$ $\operatorname{id}_{W}: W \rightarrow W$, and let $\operatorname{tr}_{R G}$ be defined as in Definition 7.2.1.

1. Let $u: P \rightarrow Q$ and $v: Q \rightarrow \operatorname{res}_{\phi} P$ be $R G$-maps of finitely generated projective $R G$-modules. Then

$$
\operatorname{tr}_{R G}(v \circ u)=\operatorname{tr}_{R G}\left(\operatorname{res}_{\phi}(u) \circ v\right) .
$$

2. Let $P_{1}, P_{2}$ be finitely generated projective $R G$-modules. Let

$$
\left(\begin{array}{ll}
u_{11} & u_{12} \\
u_{21} & u_{22}
\end{array}\right): P_{1} \oplus P_{2} \rightarrow P_{1} \oplus P_{2}
$$

be a $\phi$-twisted endomorphism. Then

$$
\operatorname{tr}_{R G}\left(\left(\begin{array}{ll}
u_{11} & u_{12} \\
u_{21} & u_{22}
\end{array}\right)\right)=\operatorname{tr}_{R G}\left(u_{11}\right)+\operatorname{tr}_{R G}\left(u_{22}\right) .
$$

3. Let $u_{1}, u_{2}: P \rightarrow \operatorname{res}_{\phi} P$ be $\phi$-twisted endomorphisms of a finitely generated projective $R G$-module $P$ and let $r_{1}, r_{2} \in R$. Then

$$
\operatorname{tr}_{R G}\left(r_{1} \cdot u_{1}+r_{2} \cdot u_{2}\right)=r_{1} \operatorname{tr}_{R G}\left(u_{1}\right)+r_{2} \operatorname{tr}_{R G}\left(u_{2}\right) .
$$

4. Let $\alpha: G \rightarrow K$ be a homomorphism of discrete group extensions with endomorphisms as in Definition 7.2.1 with $\alpha_{W}$ injective, lying in a commutative diagram

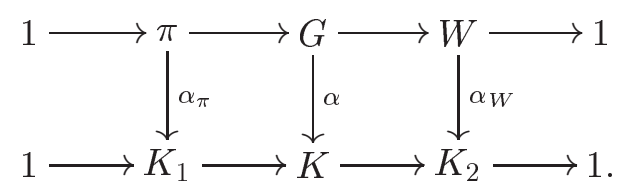

Let $u: P \rightarrow \operatorname{res}_{\phi_{G}} P$ be a $\phi_{G}$-twisted endomorphism of a finitely generated projective $R G$-module $P$. Then induction with $\alpha$ yields a $\phi_{K^{-}}$ twisted endomorphism $\alpha_{*} u$ of a finitely generated projective $R K$-module and

$$
\operatorname{tr}_{R K}\left(\alpha_{*} u\right)=\alpha_{*}^{\prime} \operatorname{tr}_{R G}(u),
$$

where $\alpha_{*}^{\prime}: R \pi_{\phi_{G}^{\prime}} \rightarrow R\left(K_{1}\right)_{\phi_{K}^{\prime}}$ is induced by $\alpha_{\pi}$. 
5. Let $\alpha: H \rightarrow G$ be an inclusion of discrete group extensions with endomorphisms as in Definition 7.2.1 with finite index $[G: H]$, lying in a commutative diagram

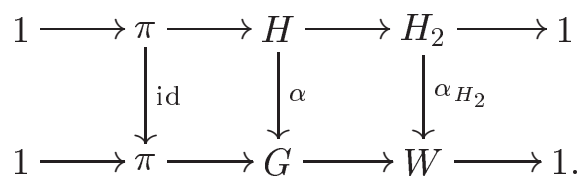

Let $u: P \rightarrow \operatorname{res}_{\phi_{G}} P$ be a $\phi_{G}$-twisted endomorphism of a finitely generated projective $R G$-module $P$. Then the restriction to $R H$ with $\alpha$ yields a $\phi_{H}$-twisted endomorphism $\alpha^{*} u$ of a finitely generated projective $R H$-module and

$$
\operatorname{id}_{*} \operatorname{tr}_{R H}\left(\alpha^{*} u\right)=[G: H] \cdot \operatorname{tr}_{R G}(u),
$$

where $\mathrm{id}_{*}: R \pi_{\phi_{H}^{\prime}} \rightarrow R \pi_{\phi_{G}^{\prime}}$ denotes the projection.

6. Let the subgroup $H \leq G$ be finite such that $|H|$ is invertible in $R$. Let $u: R[G / H] \rightarrow \operatorname{res}_{\phi} R[G / H]$ be a $\phi$-twisted endomorphism that sends $1 H$ to $\sum_{g H \in G / H} r_{g H} \cdot g H$. Then $R[G / H]$ is a finitely generated projective $R G$-module and

$$
\operatorname{tr}_{R G}(u)=|H|^{-1} \sum_{g \in \pi} r_{g H} \cdot \bar{g} \in R \pi_{\phi^{\prime}} .
$$

In particular,

$$
\operatorname{tr}_{R G}\left(\operatorname{id}_{R[G / H]}\right)=|H|^{-1} \cdot\left(\sum_{g \in \pi} 1 \cdot \bar{g}\right) \in R \pi_{\phi^{\prime}} .
$$

Proof. 1. Let $A_{u}, A_{v}$ and $A_{v \circ u}$ denote the matrices associated to $u, v$ and $v \circ u$ respectively. We use the notation $A_{i, j}=\sum_{g \in G}\left(A_{i, j}\right)_{g} \cdot g \in R G$ and calculate

$$
\begin{aligned}
\operatorname{tr}_{R G}(v \circ u) & =\sum_{i \in I} \operatorname{tr}_{R G}\left(\left(A_{v \circ u}\right)_{i i}\right) \\
& =\sum_{i, j \in I} \operatorname{tr}_{R G}\left(\left(A_{v}\right)_{i, j}\left(A_{u}\right)_{j, i}\right) \\
& =\sum_{i, j \in I} \sum_{g, g^{\prime} \in G ; g g^{\prime} \in U}\left(\left(A_{v}\right)_{i, j}\right)_{g}\left(\left(A_{u}\right)_{j, i}\right)_{g^{\prime}} \cdot \overline{g g^{\prime}} \\
& =\sum_{i, j \in I} \sum_{g, g^{\prime} \in G ; \phi\left(g^{\prime}\right) g \in U}\left(\left(A_{u}\right)_{j, i}\right)_{g^{\prime}}\left(\left(A_{v}\right)_{i, j}\right)_{g} \cdot \overline{\phi\left(g^{\prime}\right) g} \\
& =\sum_{i, j \in I} \operatorname{tr}_{R G}\left(\phi\left(\left(A_{u}\right)_{j, i}\right)\left(A_{v}\right)_{i, j}\right) \\
& =\sum_{j \in I} \operatorname{tr}_{R G}\left(\left(A_{\operatorname{res}_{\phi} u \circ v}\right)_{j, j}\right) \\
& =\operatorname{tr}_{R G}\left(\operatorname{res}_{\phi} u \circ v\right) .
\end{aligned}
$$


We used the fact that for $g, g^{\prime} \in G$ we have

$$
g g^{\prime} \in U \Leftrightarrow \phi\left(g^{\prime}\right) g \in U .
$$

If $g g^{\prime} \in \pi$, then $\phi(\gamma) g g^{\prime} \gamma^{-1} \in \pi$ for all $\gamma \in G$, and this implies $\phi\left(g^{\prime}\right) g g^{\prime}\left(g^{\prime}\right)^{-1}=\phi\left(g^{\prime}\right) g \in \pi$. Conversely, $\phi\left(g^{\prime}\right) g \in \pi$ implies that $\phi(\gamma) \phi\left(g^{\prime}\right) g \gamma^{-1} \in \pi$ for all $\gamma \in G$, and for $\gamma=g^{\prime-1}$ this gives us $\phi\left(g^{\prime-1}\right) \phi\left(g^{\prime}\right) g\left(g^{\prime-1}\right)^{-1}=g g^{\prime} \in \pi$.

2. Clear by definition.

3. Clear by definition.

4. We first check whether $\alpha_{*}^{\prime} \circ \operatorname{tr}_{R G}=\operatorname{tr}_{R K} \circ \alpha_{*}: R G \rightarrow R\left(K_{1}\right)_{\phi_{K}^{\prime}}$. It suffices to check this on a basis. Let $g \in G$. We know that $g \notin \pi$ if and only if $\operatorname{pr}_{W}(g) \neq 1_{W}$, which by injectivity of $\alpha_{W}$ is equivalent to $1_{K_{2}} \neq \alpha_{W}\left(\operatorname{pr}_{W}(g)\right)=\operatorname{pr}_{K_{2}}(\alpha(g))$, which is equivalent to $\alpha(g) \notin K_{1}$, where $\operatorname{pr}_{W}: G \rightarrow W$ and $\mathrm{pr}_{K_{2}}: K \rightarrow K_{2}$ denote the projection maps. So for $g \notin \pi$ we have $\alpha_{*}^{\prime} \operatorname{tr}_{R G}(g)=0=\operatorname{tr}_{R K}(\alpha(g))$. For $g \in \pi$ we have $\alpha_{*}^{\prime} \operatorname{tr}_{R G}(g)=\alpha_{*}^{\prime}(\bar{g})=\overline{\alpha_{\pi}(g)}=\operatorname{tr}_{R K}(\alpha(g))$. Therefore $\alpha_{*}^{\prime} \circ \operatorname{tr}_{R G}=$ $\operatorname{tr}_{R K} \circ \alpha_{*}$ on all elements of $G$, and by linearity we obtain the result for all $u \in \phi_{G}$-end $\mathrm{fp} R G_{\mathrm{fp}}$ since $A_{\alpha_{*} u}=\alpha_{*}\left(A_{u}\right)$.

5. Let $\left(A_{u}\right)_{i i}=\sum_{g \in G} n_{i g} \cdot g$. We calculate

$$
\begin{aligned}
\operatorname{id}_{*} \circ \operatorname{tr}_{R H}\left(\alpha^{*} u\right) & =\sum_{i \in I} \operatorname{id}_{*} \circ \operatorname{tr}_{R H}\left(\alpha^{*}\left(\sum_{g \in G} n_{i g} \cdot g\right)\right) \\
& =\sum_{i \in I} \operatorname{id}_{*} \circ \operatorname{tr}_{R H}\left([G: H] \sum_{h \in H} n_{i \alpha(h)} \cdot h\right) \\
& =[G: H] \sum_{i \in I} \operatorname{id}_{*} \sum_{h \in \pi} n_{i \alpha(h)} \cdot \bar{h} \\
& =[G: H] \sum_{i \in I} \sum_{g \in \pi} n_{i g} \cdot \bar{g} \\
& =[G: H] \operatorname{tr}_{R G}(u) .
\end{aligned}
$$

6. We are given

$$
u: 1 H \mapsto \sum_{g H \in G / H} r_{g H} \cdot g H=|H|^{-1}\left(\sum_{g \in G} r_{g H} \cdot g\right) H .
$$

This implies

$$
\operatorname{tr}_{R G}(u)=|H|^{-1} \operatorname{tr}_{R G}\left(\sum_{g \in G} r_{g H} \cdot g\right)=|H|^{-1} \sum_{g \in \pi} r_{g H} \cdot \bar{g} .
$$


Remark 7.2.3. In the verification of the trace property given in part 1 of Lemma 7.2.2, it is important that we work in $R \pi_{\phi^{\prime}}$ and not in $R \pi_{\phi}$ since the $g^{\prime}$ appearing in the proof can lie in $G$, not only in $\pi$.

By assertions 1 and 2 of Lemma 7.2.2, the trace map $\operatorname{tr}_{R G}$ is compatible with the relations defining $K_{0}\left(\phi-\operatorname{end}_{\mathrm{fp} R G}\right)$. So we can use its value on any representative and define

$$
\begin{aligned}
\operatorname{tr}_{R G}: K_{0}\left(\phi-\operatorname{end}_{\mathrm{fp} R G}\right) & \rightarrow R \pi_{\phi^{\prime}} \\
{[u] } & \mapsto \operatorname{tr}_{R G}(u) .
\end{aligned}
$$

Remark 7.2.4. The trace map $\operatorname{tr}_{R G}$ can be seen as a variation of a trace map between $K$-theory and Hochschild homology with coefficients in the bimodule $M_{\phi}=R G(\phi(?), ? ?)$. There is a $\operatorname{trace} \operatorname{map} \operatorname{tr}_{(K \rightarrow H H)}: K\left(R G ; M_{\phi}\right) \rightarrow$ $H H\left(R G ; M_{\phi}\right)$. One can see that $H H_{0}\left(R G ; M_{\phi}\right) \cong R G_{\phi}$, where we define $R G_{\phi}:=R G / \phi(\gamma) \beta \gamma^{-1} \sim \beta$ for $\gamma, \beta \in G$. We have an inclusion $R \pi \rightarrow R G$ of group rings. This inclusion is respected by the $G$-action given by twisted conjugation since $\beta \in \pi$ implies $\operatorname{pr}_{W}\left(\phi(\gamma) \beta \gamma^{-1}\right)=\operatorname{pr}_{W}(\gamma) \cdot 1 \cdot \operatorname{pr}_{W}\left(\gamma^{-1}\right)=1$, so $\phi(\gamma) \beta \gamma^{-1} \in \pi$ for all $\gamma \in G$. It induces an inclusion $R \pi_{\phi^{\prime}} \rightarrow R G_{\phi}$ as a direct summand. Denoting the restriction to this summand by $r_{\pi}$, one can check that $\operatorname{tr}_{R G}=r_{\pi} \circ \operatorname{tr}_{(K \rightarrow H H)_{0}}$. It would be worthwhile to develop these ideas thoroughly; one can for example study geometric interpretations and applications of the trace map $\operatorname{tr}_{(K \rightarrow H H)}$ on higher levels. But this is beyond the scope of this thesis.

\subsection{The Refined Equivariant Lefschetz Number}

We are now ready to generalize the definition of the orbifold Lefschetz number [LR03, Definition 1.4]. Let $G$ be a discrete group extension with endomorphism $\phi$ as in definition 7.2.1, and let $(X, A)$ be a finite proper relative $G$-CW-complex. Let $R$ be a commutative ring such that the order of the isotropy group $\left|G_{x}\right|$ is invertible in $R$ for every $x \in X \backslash A$. Then the cellular $R G$-chain-complex $C^{c}(X, A)$ is finite projective. Let $\left(f, f_{0}\right):(X, A) \rightarrow$ $(X, A)$ be a $\phi$-twisted cellular endomorphism. This induces a $\phi$-twisted endomorphism $C^{c}\left(f, f_{0}\right): C^{c}(X, A) \rightarrow \operatorname{res}_{\phi} C^{c}(X, A)$ of the cellular chain complex.

Definition 7.3.1. With notation as above, we define the refined equivariant Lefschetz number of $\left(f, f_{0}\right)$ to be

$$
L^{R G}\left(f, f_{0}\right):=\sum_{p \geq 0}(-1)^{p} \operatorname{tr}_{R G}\left(C_{p}^{c}\left(f, f_{0}\right)\right) \in R \pi_{\phi^{\prime}} .
$$

Writing $\left[C^{c}\left(f, f_{0}\right)\right]:=\sum_{p \geq 0}(-1)^{p}\left[C_{p}^{c}\left(f, f_{0}\right)\right] \in K_{0}\left(\phi-\operatorname{end}_{\mathrm{fp} R G}\right)$, this is

$$
L^{R G}\left(f, f_{0}\right):=\operatorname{tr}_{R G}\left(\left[C^{c}\left(f, f_{0}\right)\right]\right) \in R \pi_{\phi^{\prime}} .
$$


Using Lemma 7.2.2, we can prove an analog to a lemma of Lück and Rosenberg [LR03, Lemma 1.6]:

Lemma 7.3.2. Let $G$ be a discrete group extension with endomorphism $\phi$ as in Definition 7.2.1. Let $\left(f, f_{0}\right):(X, A) \rightarrow(X, A)$ be a $\phi$-twisted cellular endomorphism of a finite proper relative $G$ - $C W$-complex such that the order of the isotropy group $\left|G_{x}\right|$ is invertible in $R$ for every $x \in X \backslash A$. Then:

1. The refined equivariant Lefschetz number $L^{R G}\left(f, f_{0}\right)$ depends only on the $G$-homotopy class of $\left(f, f_{0}\right)$.

2. Let $\left(g, g_{0}\right):(X, A) \rightarrow(Y, B)$ and $\left(h, h_{0}\right):(Y, B) \rightarrow(X, A)$ be cellular maps of finite proper relative $G$-CW-complexes which induce $G$ maps $C^{c}\left(g, g_{0}\right): C^{c}(X, A) \rightarrow C^{c}(Y, B)$ and $C^{c}\left(h, h_{0}\right): C^{c}(Y, B) \rightarrow$ $\operatorname{res}_{\phi} C^{c}(X, A)$ and such that $\left|G_{x}\right|^{-1} \in R$ and $\left|G_{y}\right|^{-1} \in R$ for every $x \in X \backslash A$ and $y \in Y \backslash B$. Then

$$
L^{R G}\left(g \circ h, g_{0} \circ h_{0}\right)=L^{R G}\left(h \circ g, h_{0} \circ g_{0}\right) .
$$

3. Let $\alpha: G \rightarrow K$ be a homomorphism of discrete group extensions with endomorphisms as in Definition 7.2.1 such that $\alpha_{W}$ is injective, lying in a commutative diagram

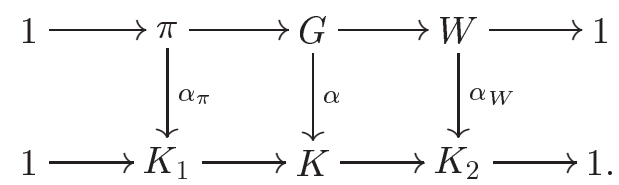

Then induction with $\alpha$ yields a cellular $K$-endomorphism $\alpha_{*}\left(f, f_{0}\right)$ of a finite proper relative $K-C W$-complex, and

$$
L^{R K}\left(\alpha_{*}\left(f, f_{0}\right)\right)=\alpha_{*}^{\prime} L^{R G}\left(f, f_{0}\right)
$$

4. Let $\alpha: H \rightarrow G$ be an inclusion of discrete group extensions with endomorphisms as in Definition 7.2.1 with finite index $[G: H]$ lying in a commutative diagram

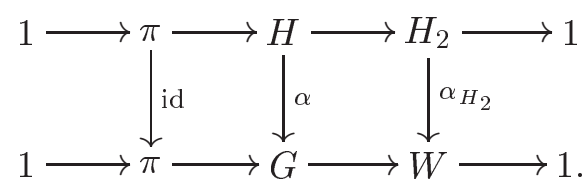

Then restriction with $\alpha$ yields a cellular endomorphism $\alpha^{*}\left(f, f_{0}\right)$ of a finite proper relative $H$-CW-complex which induces a $\phi_{H}$-twisted endomorphism $\alpha^{*}\left(C^{c}\left(f, f_{0}\right)\right): \operatorname{res}_{\alpha} C^{c}(X, A) \rightarrow \operatorname{res}_{\phi_{H}} \operatorname{res}_{\alpha} C^{c}(X, A)$ and

$$
\operatorname{id}_{*} L^{R H}\left(\alpha^{*}\left(f, f_{0}\right)\right)=[G: H] \cdot L^{R G}\left(f, f_{0}\right),
$$

where $\mathrm{id}_{*}: R \pi_{\phi_{H}^{\prime}} \rightarrow R \pi_{\phi^{\prime}}$ denotes the projection. 
Proof. 1. Let $\left(f^{\prime}, f_{0}^{\prime}\right):(X, A) \rightarrow(X, A)$ be a $\phi$-twisted cellular endomorphism which is $G$-homotopic to $\left(f, f_{0}\right)$. We have $\left[C^{c}\left(f^{\prime}, f_{0}^{\prime}\right)\right]=$ $\left[C^{c}\left(f, f_{0}\right)\right] \in K_{0}(\phi$-end $\mathrm{ff} R G)$, and so

$$
L^{R G}\left(f^{\prime}, f_{0}^{\prime}\right)=\operatorname{tr}_{R G}\left(\left[C^{c}\left(f^{\prime}, f_{0}^{\prime}\right)\right]\right)=\operatorname{tr}_{R G}\left(\left[C^{c}\left(f, f_{0}\right)\right]\right)=L^{R G}\left(f, f_{0}\right) .
$$

2. We apply assertion 1 of Lemma 7.2.2 to obtain

$$
\begin{aligned}
L^{R G}\left(g \circ h, g_{0} \circ h_{0}\right) & =\operatorname{tr}_{R G}\left(\left[C^{c}\left(g \circ h, g_{0} \circ h_{0}\right)\right]\right) \\
& =\operatorname{tr}_{R G}\left(\left[\operatorname{res}_{\phi} C^{c}\left(g, g_{0}\right)\right] \circ\left[C^{c}\left(h, h_{0}\right)\right]\right) \\
& =\operatorname{tr}_{R G}\left(\left[C^{c}\left(h, h_{0}\right)\right] \circ\left[C^{c}\left(g, g_{0}\right)\right]\right) \\
& =\operatorname{tr}_{R G}\left(\left[C^{c}\left(h \circ g, h_{0} \circ g_{0}\right)\right]\right) \\
& =L^{R G}\left(h \circ g, h_{0} \circ g_{0}\right) .
\end{aligned}
$$

3. We apply assertion 4 of Lemma 7.2 .2 to obtain

$$
\begin{aligned}
L^{R K}\left(\alpha_{*}\left(f, f_{0}\right)\right) & =\operatorname{tr}_{R K}\left(\alpha_{*}\left[C^{c}\left(f, f_{0}\right)\right]\right) \\
& =\alpha_{*}^{\prime} \operatorname{tr}_{R G}\left(\left[C^{c}\left(f, f_{0}\right)\right]\right) \\
& =\alpha_{*}^{\prime} L^{R G}\left(f, f_{0}\right) .
\end{aligned}
$$

4. We apply assertion 5 of Lemma 7.2.2 to obtain

$$
\begin{aligned}
\operatorname{id}_{*} L^{R H}\left(\alpha^{*}\left(f, f_{0}\right)\right) & =\operatorname{id}_{*} \operatorname{tr}_{R H}\left(\alpha^{*}\left[C^{c}\left(f, f_{0}\right)\right]\right) \\
& =[G: H] \cdot \operatorname{tr}_{R G}\left(\left[C^{c}\left(f, f_{0}\right)\right]\right) \\
& =[G: H] \cdot L^{R G}\left(f, f_{0}\right) .
\end{aligned}
$$

We have a refinement of the incidence number [LR03, Equation 1.8].

Definition 7.3.3. Let $G$ be a discrete group extension with endomorphism $\phi$ as in Definition 7.2.1, let $(X, A)$ be a finite proper relative $G$-CW-complex, let $e \in I_{p}(X, A)$ be a $p$-cell and let $\left(f, f_{0}\right):(X, A) \rightarrow(X, A)$ be a $\phi$-twisted cellular endomorphism. We define the refined incidence number $\operatorname{inc}_{\phi}(f, e) \in$ $\mathbb{Z} \pi_{\phi^{\prime}}$ for a $p$-cell $e \in I_{p}(X, A)$ to be the "degree" of the composition

$$
\begin{aligned}
\bar{e} / \partial e & \stackrel{i_{e}}{\longrightarrow} \bigvee_{e^{\prime} \in I_{p}(X, A)} \overline{e^{\prime}} / \partial e^{\prime} \stackrel{h \sim}{\longrightarrow} X_{p} / X_{p-1} \stackrel{f}{\rightarrow} X_{p} / X_{p-1} \stackrel{h^{-1} \sim}{\longrightarrow} \bigvee_{e^{\prime} \in I_{p}(X, A)} \overline{e^{\prime}} / \partial e^{\prime} \\
& \stackrel{\operatorname{pr}_{\pi \cdot \bar{e} / \partial e}}{\longrightarrow} \pi \cdot \bar{e} / \partial e \stackrel{\dot{\rightarrow}}{\rightarrow} \pi_{\phi^{\prime}} \cdot \bar{e} / \partial e .
\end{aligned}
$$

We have $\operatorname{inc}_{\phi}(f, e)=\operatorname{inc}_{\phi}(f, g e)$ for all $g \in G$, this is ensured by using $R \pi_{\phi^{\prime}}$. We have the equation

$$
\operatorname{inc}_{\phi}(f, e)=\sum_{\alpha \in \pi} \operatorname{inc}\left(\alpha^{-1} f, e\right) \cdot \bar{\alpha}
$$

where the incidence number [LR03, Equation 1.8] appears on the right hand side. 
Remark 7.3.4. Let $e \in I_{p}(X, A)$ be a $p$-cell. Then $C^{c}(X, A)_{e}$ is the chain complex concentrated in degree $p$ with $C_{p}^{c}(X, A)_{e}=R\left[G / G_{e}\right]$. If

$$
\left.C_{p}^{c}\left(f, f_{0}\right)\right|_{e}=\sum_{g G_{e} \in G / G_{e}} r_{g G_{e}} \cdot g G_{e}: R\left[G / G_{e}\right] \rightarrow \operatorname{res}_{\phi} R\left[G / G_{e}\right]
$$

then $\operatorname{inc}_{\phi}(f, e)=\sum_{g \in \pi} r_{g G_{e}} \cdot \bar{g}$, and by assertion 5 of Lemma 7.2.2 we have

$$
\operatorname{tr}_{R G}\left(\left.C_{p}^{c}\left(f, f_{0}\right)\right|_{e}\right)=\left|G_{e}\right|^{-1} \sum_{g \in \pi} r_{g G_{e}} \cdot \bar{g}=\left|G_{e}\right|^{-1} \operatorname{inc}_{\phi}(f, e) .
$$

The observation in Remark 7.3.4 helps us prove the following analog of a result by Lück and Rosenberg [LR03, Lemma 1.9].

Lemma 7.3.5. Let $G$ be a discrete group extension with endomorphism $\phi$ as in Definition 7.2.1. Let $(X, A)$ be a finite proper relative $G$ - $C W$-complex. Let $R$ be a commutative ring such that the order of the isotropy group $\left|G_{x}\right|$ is invertible in $R$ for every $x \in X \backslash A$. Let $\left(f, f_{0}\right):(X, A) \rightarrow(X, A)$ be a $\phi$-twisted cellular endomorphism. Then

$$
L^{R G}\left(f, f_{0}\right)=\sum_{p \geq 0}(-1)^{p} \sum_{G \cdot e \in G \backslash I_{p}(X, A)}\left|G_{e}\right|^{-1} \cdot \operatorname{inc}_{\phi}(f, e) \in R \pi_{\phi^{\prime}} .
$$

Proof. We calculate

$$
\begin{aligned}
L^{R G}\left(f, f_{0}\right) & =\sum_{p \geq 0}(-1)^{p} \operatorname{tr}_{R G}\left(C_{p}\left(f, f_{0}\right)\right) \\
& =\sum_{p \geq 0}(-1)^{p} \sum_{G \cdot e \in G \backslash I_{p}(X, A)} \operatorname{tr}_{R G}\left(\left.C_{p}\left(f, f_{0}\right)\right|_{e}\right) \\
& =\sum_{p \geq 0}(-1)^{p} \sum_{G \cdot e \in G \backslash I_{p}(X, A)}\left|G_{e}\right|^{-1} \cdot \operatorname{inc}_{\phi}(f, e) .
\end{aligned}
$$

As already mentioned, we will apply the above definitions and results to our geometrical considerations: For $x \in \Pi(G, X)$ with $X^{H}(f(x))=X^{H}(x)$, the group $\operatorname{Aut}(x)$ is of the type considered in Definition 7.2.1, it is a discrete group extension $1 \rightarrow \pi_{1}\left(X^{H}(x), x\right) \rightarrow \operatorname{Aut}(x) \rightarrow W H_{x} \rightarrow 1$ with endomorphism $\phi$ which restricts to $\pi_{1}\left(X^{H}(x), x\right)$ as $\phi_{\pi_{1}\left(X^{H}(x), x\right)}=c_{w_{x}} \circ \pi_{1}\left(f^{H}(x), x\right)$ and such that $\phi_{W}=\operatorname{id}_{W H_{x}}: W H_{x} \rightarrow W H_{x}$.

\subsection{The Generalized Trace Map}

In this section, we define a trace map $\operatorname{tr}_{G(X, f)}$ on the abelian group $U_{G}^{\mathbb{Z}}(X, f)$, for $(X, f) \in \operatorname{End}\left(G-\mathcal{C} W_{\mathrm{fp}}\right)$, for discrete groups $G$. So $X$ is a finite proper $G$-CW-complex and $f: X \rightarrow X$ is a $G$-equivariant endomorphism. The trace map $\operatorname{tr}_{G(X, f)}$ maps $U_{G}^{\mathbb{Z}}(X, f)$ to the abelian group $\Lambda_{G}(X, f)$ defined in 
Definition 7.1.8. It will turn out to map the universal functorial equivariant Lefschetz invariant $\left(U_{G}^{\mathbb{Z}}(X, f), u_{G}^{\mathbb{Z}}(X, f)\right)$ to the generalized equivariant Lefschetz invariant $\left(\Lambda_{G}(X, f), \lambda_{G}(f)\right)$ which will be defined in Definition 7.5.1.

By the splitting result from Section 6.2, the universal functorial equivariant Lefschetz invariant $\left(U_{G}^{\mathbb{Z}}(X, f), u_{G}^{\mathbb{Z}}(X, f)\right)$ can be written as

$$
\begin{aligned}
& U_{G}^{\mathbb{Z}}(X, f) \stackrel{\sim}{\rightarrow} \bigoplus_{\substack{\bar{x} \in \operatorname{Is} \Pi(G, X), X^{H}(f(x))=X^{H}(x)}} U_{G}^{\mathbb{Z}}(X, f)_{\bar{x}} \oplus \text { other terms } \\
& u_{G}^{\mathbb{Z}}(X, f) \mapsto \sum_{\substack{\bar{x} \in \operatorname{Is} \Pi(G, X), X^{H}(f(x))=X^{H}(x)}}\left[C^{c}\left(\left.\widetilde{f}\right|_{\left(\widetilde{X^{H}}(x), X^{>H}(x)\right)}\right)\right]+\text { other terms, }
\end{aligned}
$$

where

$$
U_{G}^{\mathbb{Z}}(X, f)_{\bar{x}}:=\coprod_{\substack{x \in \bar{x}, w_{x} \in \operatorname{Mor}(x, f(x))}} K_{0}\left(\phi_{x, w_{x}}-\operatorname{end}_{\mathrm{ff} \mathbb{Z} \operatorname{Aut}(x)}\right) / \sim .
$$

Here the group elements $g_{w_{x}}$ introduced in Section 6.2 need not appear in the term $\left[C^{c}\left(\left.\widetilde{f}\right|_{\left(\widetilde{X^{H}(x)}, X^{>H}(x)\right)}\right)\right]$ since we can choose a morphism $w_{x}$ of the form $w_{x}=\left(\mathrm{id},\left[v_{x}\right]\right)$. In Section 7.1, we defined the abelian group $\Lambda_{G}(X, f)$. This is the target of our trace map. We define $\operatorname{tr}_{G(X, f)}$ as a group homomorphism from $U_{G}^{\mathbb{Z}}(X, f)$ to $\Lambda_{G}(X, f)$, or, more precisely, as a collection of group homomorphisms, one for each $\bar{x} \in \operatorname{Is} \Pi(G, X)$ with $X^{H}(f(x))=X^{H}(x)$. For $x \in \mathrm{Ob} \Pi(G, X)$ with $X^{H}(f(x))=X^{H}(x)$ and a morphism $w_{x}=\left(\mathrm{id},\left[v_{x}\right]\right) \in$ $\operatorname{Mor}(f(x), x)$, we set

$$
\operatorname{tr}_{G(X, f)_{x}}:=\operatorname{tr}_{\mathbb{Z} \operatorname{Aut}(x)}: K_{0}\left(\phi_{x, w_{x}}-\operatorname{end}_{\mathrm{ff} \mathbb{Z} \operatorname{Aut}(x)}\right) \rightarrow \mathbb{Z} \pi_{1}\left(X^{H}(x), x\right)_{\phi_{x, w_{x}}^{\prime}} .
$$

We want this definition to be independent of the choice of the element $x \in \bar{x}$ and the morphism $w_{x}$. We use the following lemma.

Lemma 7.4.1. Let $(\sigma,[t]) \in \operatorname{Mor}_{\Pi(G, X)}(x, y)$ be an isomorphism from $x$ to $y$, let $w_{x}=\left(\mathrm{id},\left[v_{x}\right]\right) \in \operatorname{Mor}(f(x), x)$ and $w_{y}=\left(\mathrm{id},\left[v_{y}\right]\right) \in \operatorname{Mor}(f(y), y)$. Then the diagram

$$
\begin{aligned}
& K_{0}\left(\phi_{y, w_{y}}-\operatorname{end}_{\mathrm{ff} \mathbb{Z} \operatorname{Aut}(y)}\right) \stackrel{\operatorname{tr}_{\mathbb{Z} \operatorname{Aut}(y)}}{\longrightarrow} \mathbb{Z} \pi_{1}\left(X^{H}(x), x\right)_{\phi_{y, w_{y}}^{\prime}} \\
& \downarrow(\sigma,[t])_{w_{x}, w_{y}}^{*} \downarrow(\sigma,[t])_{w_{x}, w_{y}}^{*} \\
& K_{0}\left(\phi_{x, w_{x}}-\operatorname{end}_{\mathrm{ffZ} \mathbb{Z} \operatorname{Aut}(x)}\right) \stackrel{\operatorname{tr}_{\mathbb{Z} \operatorname{Aut}(x)}}{\longrightarrow} \mathbb{Z} \pi_{1}\left(X^{H}(x), x\right)_{\phi_{x, w_{x}}^{\prime}} .
\end{aligned}
$$

commutes.

Proof. The isomorphism $(\sigma,[t])$ induces the isomorphism

$$
\begin{aligned}
(\sigma,[t])_{w_{x}, w_{y}}^{*}: \mathbb{Z} \operatorname{Aut}(y) & \stackrel{\sim}{\rightarrow} \mathbb{Z} \operatorname{Aut}(x) \\
\sum_{g \in \operatorname{Aut}(y)} n_{g} \cdot g & \mapsto \sum_{g \in \operatorname{Aut}(y)} n_{g} \cdot(\sigma,[t])_{w_{x}, w_{y}}^{*}(g)
\end{aligned}
$$


where for $g=\left(\tau,\left[v_{g}\right]\right) \in \operatorname{Aut}(y)$ we set

$$
(\sigma,[t])_{w_{x}, w_{y}}^{*}(g):=w_{x} f\left((\sigma,[t])^{-1}\right) w_{y}^{-1} g(\sigma,[t]) \in \operatorname{Aut}(x) .
$$

This in turn induces the isomorphism

$$
(\sigma,[t])_{w_{x}, w_{y}}^{*}: K_{0}\left(\phi_{y, w_{y}}-\operatorname{end}_{\mathrm{ffZ} \mathbb{A u t}(y)}\right) \stackrel{\sim}{\rightarrow} K_{0}\left(\phi_{x, w_{x}}-\operatorname{end}_{\mathrm{ffZ} \mathbb{A u t}(x)}\right)
$$

that was called $K_{0}\left(R_{x, w_{x}} I_{y, w_{y}}\right)$ in Section 6.1 (suppressing the choice of $(\sigma,[t])$, which by Lemma 7.1.5 does not play a role anyway).

The map $(\sigma,[t])_{w_{x}, w_{y}}^{*}$ maps the subgroup $\mathbb{Z} \pi_{1}\left(X^{H}(y), y\right) \leq \mathbb{Z} \operatorname{Aut}(y)$ isomorphically to $\mathbb{Z} \pi_{1}\left(X^{H}(x), x\right) \leq \mathbb{Z} \operatorname{Aut}(x)$. The restriction of $(\sigma,[t])_{w_{x}, w_{y}}^{*}$ to $\mathbb{Z} \pi_{1}\left(X^{H}(y), y\right)$ is

$$
\begin{aligned}
\left.(\sigma,[t])_{w_{x}, w_{y}}^{*}\right|_{\mathbb{Z} \pi_{1}\left(X^{H}(y), y\right)}: & \\
\mathbb{Z} \pi_{1}\left(X^{H}(y), y\right) & \rightarrow \mathbb{Z} \pi_{1}\left(X^{H}(x), x\right) \\
\sum_{g \in \pi_{1}\left(X^{H}(y), y\right)} n_{g} \cdot g & \mapsto \sum_{g \in \pi_{1}\left(X^{H}(y), y\right)} n_{g} \cdot v_{x} f\left(t^{-1}\right) \sigma^{*}\left(v_{y}^{-1}\right) \sigma^{*}(g) t .
\end{aligned}
$$

We obtain $\operatorname{tr}_{\mathbb{Z} \operatorname{Aut}(x)}(\sigma,[t])_{w_{x}, w_{y}}^{*}=(\sigma,[t])_{w_{x}, w_{y}}^{*} \operatorname{tr}_{\mathbb{Z} \operatorname{Aut}(y)}$ on $\mathbb{Z} \operatorname{Aut}(y)$ since for $\sum_{g \in G} n_{g} \cdot g \in \mathbb{Z} \operatorname{Aut}(y)$ we have

$$
\begin{aligned}
& (\sigma,[t])_{w_{x}, w_{y}}^{*} \operatorname{tr}_{\mathbb{Z} \operatorname{Aut}(y)} \sum_{g \in G} n_{g} \cdot g \\
& =(\sigma,[t])_{w_{x}, w_{y}}^{*} \sum_{g \in \pi_{1}\left(X^{H}(y), y\right)} n_{g} \cdot \bar{g} \\
& =\sum_{g \in \pi_{1}\left(X^{H}(y), y\right)} n_{g} \cdot \overline{\left.(\sigma,[t])_{w_{x}, w_{y}}^{*}\right|_{\pi_{1}\left(X^{H}(y), y\right)}(g)} \\
& =\operatorname{tr}_{\mathbb{Z} \operatorname{Aut}(x)}(\sigma,[t])_{w_{x}, w_{y}}^{*} \sum_{g \in G} n_{g} \cdot g .
\end{aligned}
$$

This extends to $[u] \in K_{0}\left(\phi_{y, w_{y}}-\operatorname{end}_{\mathrm{ffZ} \operatorname{Aut}(y)}\right)$ by linearity.

In the definition of

$$
U_{G}^{\mathbb{Z}}(X, f)_{\bar{x}}:=\coprod_{\substack{x \in \bar{x}, w_{x} \in \operatorname{Mor}(f(x), x)}} K_{0}\left(\phi_{x, w_{x}}-\operatorname{end}_{\mathrm{ff} \mathbb{Z} \operatorname{Aut}(x)}\right) / \sim
$$

we were allowed to choose any morphism $w_{x}$, not just those of the special form $w_{x}=\left(\mathrm{id},\left[v_{x}\right]\right)$. We extend the definition of the trace map to the group $K_{0}\left(\phi_{x, w}\right.$-end $\left.\operatorname{ffZAut}(x)\right)$ for any morphism $w=(\sigma,[v]) \in \operatorname{Mor}(f(x), x)$ by choosing a morphism (id, $[s]) \in \operatorname{Mor}(x \circ \sigma, x)$ and composing $w$ with the morphism $\left(\sigma^{-1},\left[\sigma^{-1 *} s\right]\right) \in \operatorname{Aut}(x)$. We obtain $w^{\prime}=\left(\sigma^{-1},\left[\sigma^{-1^{*}} s\right]\right)(\sigma,[v])=$ 
(id, $[s v]) \in \operatorname{Mor}(f(x), x)$. We define the trace map on $K_{0}\left(\phi_{x, w}\right.$-end $\left.\mathrm{ff}_{\mathrm{f} \mathbb{Z} \operatorname{Aut}(x)}\right)$ to be the composition

$$
\begin{aligned}
K_{0}\left(\phi_{x, w^{-}}-\operatorname{end}_{\mathrm{ffZ} \mathbb{A u t}(x)}\right) \stackrel{\sim}{\rightarrow} K_{0}\left(\phi_{x, w^{\prime}}-\operatorname{end}_{\mathrm{ff} \mathbb{Z} \operatorname{Aut}(x)}\right) \\
\\
\stackrel{\operatorname{tr}_{\mathbb{Z} \operatorname{Aut}(x)}}{\longrightarrow} \pi_{1}\left(X^{H}(x), x\right)_{\phi_{x, w^{\prime}}^{\prime}} \\
\stackrel{\sim}{\rightarrow} \mathbb{Z} \pi_{1}\left(X^{H}(x), x\right)_{\phi_{x}^{\prime}} .
\end{aligned}
$$

This does not depend on the choice of the morphism $s$ since for another choice $\widetilde{s} \in \operatorname{Mor}(x \circ \sigma, x)$ we obtain $\widetilde{w}=(\mathrm{id},[\widetilde{s} v])=\left(\mathrm{id},\left[\widetilde{s} s^{-1}\right]\right) w^{\prime}$, and we know that $\left(\mathrm{id},\left[\widetilde{s} s^{-1}\right]\right)^{*}=\mathrm{id}_{\mathbb{Z} \pi_{1}\left(X^{H}(x), x\right)_{\phi^{\prime}}}$.

The map $\operatorname{tr}_{G(X, f)_{x}}$ induces a well-defined map $\operatorname{tr}_{G(X, f)}$ on isomorphism classes $\bar{x}$ because of Lemma 7.4.1.

Definition 7.4.2. Let $G$ be a discrete group, $X$ a finite proper $G$-CWcomplex and $f: X \rightarrow X$ a $G$-equivariant endomorphism. We define

$$
\operatorname{tr}_{G(X, f)}:=\bigoplus_{\substack{\bar{x} \in \operatorname{Is} \Pi(G, X), X^{H}(f(x))=X^{H}(x)}} \operatorname{tr}_{G(X, f)_{\bar{x}}} \oplus 0: U_{G}^{\mathbb{Z}}(X, f) \rightarrow \Lambda_{G}(X, f) .
$$

We want the maps $\operatorname{tr}_{G(X, f)}$ to form a natural transformation of families of functors from the categories $\operatorname{End}\left(G-\mathcal{C} W_{\mathrm{fp}}\right)$ of endomorphisms of finite proper $G$-CW-complexes for discrete groups $G$ to $\mathcal{A} b$. We need to endow $\Lambda$ with the structure of such a functor.

Lemma 7.4.3. The groups $\Lambda_{G}(X, f)$ are naturally endowed with the structure of a family of functors $\Lambda_{G}$ from $\operatorname{End}\left(G-\mathcal{C} W_{\mathrm{fp}}\right)$ to $\mathcal{A} b$, for discrete groups $G$, which is compatible with the induction structure.

Proof. Let $a:(X, f) \rightarrow(Y, g)$ be a $G$-equivariant map between $G$-CWcomplexes with endomorphisms. Then the map $a$ induces a functor

$$
\begin{aligned}
A=\Pi(G, a): \Pi(G, X) & \rightarrow \Pi(G, Y) \\
(x: G / H \rightarrow X) & \mapsto(a(x):=a \circ x: G / H \rightarrow Y) \\
(\sigma,[t]): x \rightarrow y & \mapsto(\sigma,[a \circ t]): a(x) \rightarrow a(y) .
\end{aligned}
$$

For any $x \in \Pi(G, X)$, the functor $A$ induces a group homomorphism $\alpha_{x}:=$ $\left.A\right|_{\text {Aut }(x)}: \operatorname{Aut}(x) \rightarrow \operatorname{Aut}(a(x))$. The homomorphism $\alpha_{x}$ can be restricted to $\pi_{1}\left(X^{H}(x), x\right)$ as $\left.\alpha_{x}\right|_{\pi_{1}\left(X^{H}(x), x\right)}=\pi_{1}\left(\left.a\right|_{X^{H}(x)}, x\right)$. The map $\pi_{1}\left(\left.a\right|_{X^{H}(x)}, x\right)$ induces a map $\left(\alpha_{x}^{\prime}\right)_{*}: R \pi_{1}\left(X^{H}(x), x\right)_{\phi_{x}^{\prime}} \rightarrow R \pi_{1}\left(Y^{H}(a(x)), a(x)\right)_{\phi_{a(x)}^{\prime}}$. We set

$$
\begin{aligned}
\Lambda_{G}(a)_{x}:=\left(\alpha_{x}^{\prime}\right)_{*}: R \pi_{1}\left(X^{H}(x), x\right)_{\phi_{x}^{\prime}} & \rightarrow R \pi_{1}\left(Y^{H}(a(x)), a(x)\right)_{\phi_{a(x)}^{\prime}} \\
\sum_{\bar{g} \in \pi_{1}\left(X^{H}(x), x\right)_{\phi_{x}^{\prime}}} r_{g} \cdot \bar{g} & \mapsto \sum_{\bar{g} \in \pi_{1}\left(X^{H}(x), x\right)_{\phi_{x}^{\prime}}} r_{g} \cdot \overline{\pi_{1}\left(\left.a\right|_{X^{H}(x)}, x\right)(g)} .
\end{aligned}
$$


Using Lemma 7.4.1, we obtain independence of the choice of basepoint $x$ and morphism $w_{x}$ and therefore an induced map

$$
\Lambda_{G}(a)_{\bar{x}}: \mathbb{Z} \Pi(G, X)_{\phi_{X}, \bar{x}} \rightarrow \mathbb{Z} \Pi(G, Y)_{\phi_{Y}, \overline{a(x)}} .
$$

We set

$$
\Lambda_{G}(a):=\left(\bigoplus_{\substack{\bar{x} \in \operatorname{Is} \Pi(G, X), X^{H}(f(x))=X^{H}(x)}} \Lambda_{G}(a)_{\bar{x}}\right) \oplus 0: \Lambda_{G}(X, f) \rightarrow \Lambda_{G}(Y, g) .
$$

It remains to show that $\Lambda$ is compatible with the induction structure in $G$. If we have an inclusion $\alpha: G \rightarrow K$, then the map $\left.\alpha\right|_{\pi_{1}\left(X^{H}(x), x\right)_{\phi_{G}^{\prime}}}$ induces a map $\alpha_{*}^{\prime}: R \pi_{1}\left(X^{H}(x), x\right)_{\phi_{G}^{\prime}} \rightarrow R \pi_{1}\left(\left(\operatorname{ind}_{\alpha} X\right)^{H}\left(\operatorname{ind}_{\alpha} x\right), \operatorname{ind}_{\alpha} x\right)_{\phi_{K}^{\prime}}$. We set $\Lambda(\alpha)_{x}=\alpha_{*}^{\prime}$, then these maps combine to form a group homomorphism $\alpha_{*}=\Lambda(\alpha): \Lambda_{G}(X, f) \rightarrow \Lambda_{K}\left(\operatorname{ind}_{\alpha} X, \operatorname{ind}_{\alpha} f\right)$.

The maps $\operatorname{tr}_{G(X, f)}$ actually form a natural transformation of families of functors from the categories $\operatorname{End}\left(G-\mathcal{C} W_{\mathrm{fp}}\right)$ of endomorphisms of finite proper $G$-CW-complexes for discrete groups $G$ to $\mathcal{A} b$.

Proposition 7.4.4. The collection of maps $\operatorname{tr}_{G(X, f)}$ is a natural transformation of families of functors from $\operatorname{End}\left(G-\mathcal{C} W_{\mathrm{fp}}\right)$ to $\mathcal{A} b$, for discrete groups $G$.

Proof. Let $a:(X, f) \rightarrow(Y, g)$ be a $G$-equivariant map between $G$-CWcomplexes with endomorphisms. Then the map $a$ induces a functor $A=$ $\Pi(G, a)$. The group homomorphism $\alpha_{x}:=\left.A\right|_{\operatorname{Aut}(x)}: \operatorname{Aut}(x) \rightarrow \operatorname{Aut}(a(x))$ lies in the commutative diagram

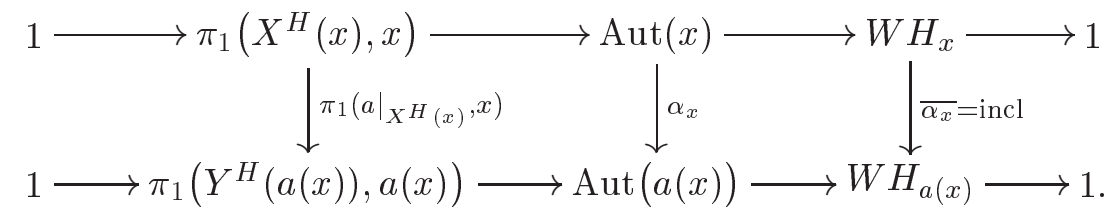

The map $\overline{\alpha_{x}}=$ incl: $W H_{x} \rightarrow W H_{a(x)}$ is an inclusion since the elements in $W H$ which fix the connected component $X^{H}(x)$ also fix the connected component $Y^{H}(a(x))$, by equivariance and continuity of $a$. Therefore we can apply Lemma 7.2.2, assertion 4 to obtain

$$
\operatorname{tr}_{R \operatorname{Aut}(a(x))}\left(\alpha_{x}\right)_{*}(u)=\left(\alpha_{x}^{\prime}\right)_{*} \operatorname{tr}_{R \operatorname{Aut}(x)}(u)
$$

for all $\left(\phi_{X}\right)_{x, w_{x}}$-twisted endomorphisms $u$ of finite projective $R \operatorname{Aut}(x)$ modules. Here $\left(\alpha_{x}^{\prime}\right)_{*}: R \pi_{1}\left(X^{H}(x), x\right)_{\phi_{x}^{\prime}} \rightarrow R \pi_{1}\left(Y^{H}(a(x)), a(x)\right)_{\phi_{a(x)}^{\prime}}$ is induced by $\pi_{1}\left(\left.a\right|_{X^{H}(x)}, x\right)$, and these maps combine to form $\Lambda_{G}(a)$ as seen in the proof of Lemma 7.4.3. 
On the other hand, taking the induction maps $K_{0}\left(\left(\alpha_{\bar{x}}\right)_{*}\right): U_{G}^{\mathbb{Z}}(X, f)_{\bar{x}} \rightarrow$ $U_{G}^{\mathbb{Z}}(Y, g) \frac{}{a(x)}$ all together gives $K_{0}$ of the induction with $A:=\Pi(G, a)$, namely $U_{G}^{\mathbb{Z}}(a)=K_{0}\left(A_{*}\right): U_{G}^{\mathbb{Z}}(X, f) \rightarrow U_{G}^{\mathbb{Z}}(Y, g)$. Combining the above equation for all $\bar{x} \in \operatorname{Is} \Pi(G, X)$, we arrive at

$$
\operatorname{tr}_{G(Y, g)} \circ U_{G}^{\mathbb{Z}}(a)(u)=\Lambda_{G}(a) \circ \operatorname{tr}_{G(X, f)}(u)
$$

for all $u \in U_{G}^{\mathbb{Z}}(X, f)$. We have shown that the diagram

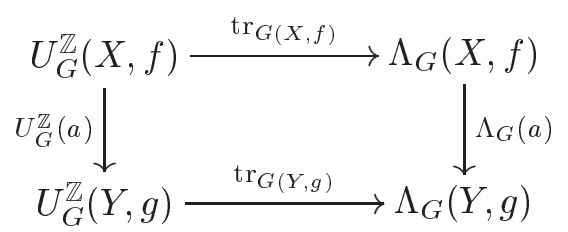

commutes, so the trace map $\operatorname{tr}_{G(X, f)}$ is a natural transformation of functors from $\operatorname{End}\left(G-\mathcal{C} W_{\text {fp }}\right)$ to $\mathcal{A} b$.

It remains to show that $\operatorname{tr}_{G(X, f)}$ is compatible with the induction structure in $G$. If we have an inclusion $\alpha: G \rightarrow K$, then by Section 3.4 the functor $\Pi\left(\operatorname{ind}_{\alpha}\right): \Pi(G, X) \rightarrow \Pi\left(K, \operatorname{ind}_{\alpha} X\right)$ induces a group homomorphism $\alpha_{*}: U_{G}^{\mathbb{Z}}(X, f) \rightarrow U_{K}^{\mathbb{Z}}\left(\operatorname{ind}_{\alpha} X, \operatorname{ind}_{\alpha} f\right)$. Let $x: G / H \rightarrow X$ be given with $X^{H}(f(x))=X^{H}(x)$. We set $W_{G} H:=N_{G} H / H$ and $W_{K} H=N_{K} H / H$. The map $\alpha: G \rightarrow K$ is injective, and so $W_{G} H \rightarrow W_{K} H$ is injective. If an element of $W_{G} H$ fixes the component $X^{H}(x)$, then its image fixes $\left(\operatorname{ind}_{\alpha} X\right)^{H}\left(\operatorname{ind}_{\alpha} x\right)$. This implies that $\left.\alpha\right|_{N_{G} H}: N_{G} H \rightarrow N_{K} H$ is injective. So the map $\left.\Pi\left(\operatorname{ind}_{\alpha}\right)\right|_{\operatorname{Aut}(x)}: \operatorname{Aut}(x) \rightarrow \operatorname{Aut}\left(\operatorname{ind}_{\alpha} x\right)$ induces an injective map $\left.\Pi\left(\operatorname{ind}_{\alpha}\right)\right|_{W_{G} H_{x}}: W_{G} H_{x} \rightarrow W_{K} H_{\operatorname{ind}_{\alpha}(x)}$. We can apply assertion 4 of Lemma 7.2.2 to obtain $\operatorname{tr}_{R K} \alpha_{*} u=\alpha_{*}^{\prime} \operatorname{tr}_{R G} u$ for $u \in \phi_{G}$-end $\operatorname{fp}_{\mathrm{fp}}$, where $\alpha_{*}^{\prime}: R \pi_{1}\left(X^{H}(x), x\right)_{\phi_{G}^{\prime}} \rightarrow R \pi_{1}\left(\left(\operatorname{ind}_{\alpha} X\right)^{H}\left(\operatorname{ind}_{\alpha} x\right), \operatorname{ind}_{\alpha} x\right)_{\phi_{K}^{\prime}}$ is induced by $\left.\alpha\right|_{\pi_{1}\left(X^{H}(x), x\right)_{\phi_{G}^{\prime}}}$. Since $\Lambda(\alpha)_{x}$ is defined to be $\alpha_{*}^{\prime}$, these maps combine to form $\alpha_{*}: \Lambda_{G}(X, f) \rightarrow \Lambda_{K}\left(\operatorname{ind}_{\alpha} X, \operatorname{ind}_{\alpha} f\right)$ such that the desired equation $\operatorname{tr}_{K\left(\operatorname{ind}_{\alpha} X, \operatorname{ind}_{\alpha} f\right)} \alpha_{*}=\alpha_{*} \operatorname{tr}_{G(X, f)}$ holds on $U_{G}^{\mathbb{Z}}(X, f)$.

\subsection{The Generalized Equivariant Lefschetz Invari- ant}

In this section, we define the invariant which contains the fixed point information we are interested in, an equivariant analog of the generalized Lefschetz invariant [Rei38, Wec41] and a refinement of the equivariant Lefschetz class [LR03, Definition 3.6]. It takes its values in the abelian group $\Lambda_{G}(X, f)$, which was defined in Definition 7.1.8.

Definition 7.5.1. Let $G$ be a discrete group, let $X$ be a finite proper $G$ CW-complex, and let $f: X \rightarrow X$ be a $G$-equivariant cellular endomorphism. 
We define the generalized equivariant Lefschetz invariant of $f$,

$$
\lambda_{G}(f) \in \Lambda_{G}(X, f),
$$

by

$$
\lambda_{G}(f)_{\bar{x}}:= \begin{cases}L^{\mathbb{Z} \operatorname{Aut}(x)}\left(\widetilde{f^{H}(x)}, \widetilde{f>H}(x)\right) & \text { if } X^{H}(f(x))=X^{H}(x) \\ 0 & \text { else. }\end{cases}
$$

Here we consider $L^{\mathbb{Z} \operatorname{Aut}(x)}\left(\widetilde{f^{H}(x)}, \widehat{f^{>H}(x)}\right) \in \mathbb{Z} \pi_{1}\left(X^{H}(x), x\right)_{\phi_{x, w_{x}}^{\prime}}$ as an element in $\mathbb{Z} \Pi(G, X)_{\phi, \bar{x}}$ via the canonical isomorphism $\psi_{x} \psi_{w_{x}}$ induced by the inclusion. We can use $\mathbb{Z}$ in this definition because $\operatorname{Aut}(x)$ operates freely on $\widehat{X^{H}(x)} \backslash \widehat{X^{>H}(x)}$. For $\lambda_{G}(f)$ to be well-defined we need to show that the element in $\mathbb{Z} \Pi(G, X)_{\phi, \bar{x}}$ defined by $\left.L^{\mathbb{Z} \operatorname{Aut}(x)} \widehat{\left(f^{H}(x)\right.}, \widehat{f^{>H}(x)}\right)$ only depends on the isomorphism class of $x$, i.e., that for any isomorphism $(\sigma,[t]) \in$ $\operatorname{Mor}_{\Pi(G, X)}\left(x, x^{\prime}\right)$ we have

$$
(\sigma,[t])^{*}\left(L^{\mathbb{Z} \operatorname{Aut}\left(x^{\prime}\right)}\left(\widetilde{f^{H}\left(x^{\prime}\right)}, \widehat{f^{>H}\left(x^{\prime}\right)}\right)\right)=L^{\mathbb{Z} \operatorname{Aut}(x)}\left(\widetilde{f^{H}(x)}, \widehat{f^{>H}(x)}\right) .
$$

We first observe that

$$
(\sigma,[t])_{w_{x}, w_{x}^{\prime}}^{*}\left(C^{c}\left(\widetilde{f^{H}\left(x^{\prime}\right)}, \widehat{f^{>H}\left(x^{\prime}\right)}\right)\right)=\left(C^{c}\left(\widetilde{f^{H}(x)}, \widehat{f^{>H}(x)}\right)\right) .
$$

We choose a $p$-cell $e \in\left(\widetilde{f^{H}\left(x^{\prime}\right)}, \widetilde{f^{>H}\left(x^{\prime}\right)}\right)$. The map $C_{p}^{c}\left(\widetilde{f^{H}\left(x^{\prime}\right)}, \widetilde{f^{>H}\left(x^{\prime}\right)}\right)$ maps $1 \cdot e$ to $\sum_{i \in I_{p}} n_{i} g_{i} \cdot e_{i, p}$ with $g_{i} \in \operatorname{Aut}\left(x^{\prime}\right)$. Cells in $X^{H}\left(x^{\prime}\right)$ are given by morphisms from $x^{\prime}$ to their centers, so cells in $X^{H}(x)$ can be given by the same morphisms composed with the morphism $(\sigma,[t])$ from $x$ to $x^{\prime}$. Writing out the map with respect to this new basis $\left\{e_{i, p}^{\prime}\right\}_{i \in I_{p}}$ gives us $\left.C_{p}^{c}\left(\widehat{f^{H}(x)}, \widehat{f>H(x)}\right)\right|_{e^{\prime}}$. One obtains $1 \cdot e^{\prime} \mapsto \sum_{i \in I_{p}} n_{i}(\sigma,[t])^{*}\left(g_{i}\right) \cdot e_{i, p}^{\prime}$, and this is indeed $(\sigma,[t])_{w_{x}, w_{x}^{\prime}}^{*}\left(\left.C_{p}^{c}\left(\widetilde{f^{H}\left(x^{\prime}\right)}, \widehat{f^{>H}\left(x^{\prime}\right)}\right)\right|_{e}\right)$.

Thus we deduce from Lemma 7.4.1 that

$$
\begin{aligned}
(\sigma, & ,[t])^{*} L^{\mathbb{Z} \operatorname{Aut}\left(x^{\prime}\right)}\left(\widehat{f^{H}\left(x^{\prime}\right)}, \widehat{f^{>H}\left(x^{\prime}\right)}\right) \\
& =(\sigma,[t])^{*} \operatorname{tr}_{\mathbb{Z} \operatorname{Aut}\left(x^{\prime}\right)}\left(\left[C^{c}\left(\widehat{f^{H}\left(x^{\prime}\right)}, \widehat{f^{>H}\left(x^{\prime}\right)}\right)\right]\right) \\
& =\operatorname{tr}_{\mathbb{Z} \operatorname{Aut}(x)}(\sigma,[t])^{*}\left(\left[C^{c}\left(\widehat{f^{H}\left(x^{\prime}\right)}, \widehat{f^{>H}\left(x^{\prime}\right)}\right)\right]\right) \\
& =\operatorname{tr}_{\mathbb{Z} \operatorname{Aut}(x)}\left(\left[C^{c}\left(\widehat{f^{H}(x)}, \widehat{f^{>H}(x)}\right)\right]\right) \\
& =L^{\mathbb{Z} \operatorname{Aut}(x)}\left(\widehat{f^{H}(x)}, \widehat{f^{>H}(x)}\right) .
\end{aligned}
$$


What happens when we apply the trace map $\operatorname{tr}_{G(X, f)}$ to the universal functorial equivariant Lefschetz invariant $u_{G}^{\mathbb{Z}}(X, f) \in U_{G}^{\mathbb{Z}}(X, f)$ ? We see that

$$
\begin{aligned}
& \operatorname{tr}_{G(X, f)}\left(u_{G}^{\mathbb{Z}}(X, f)\right) \\
& =\sum_{\substack{\bar{x} \in \operatorname{Is} \Pi(G, X), X^{H}(f(x))=X^{H}(x)}} \operatorname{tr}_{G(X, f) \bar{x}}\left(\left[C^{c}\left(\left.\widetilde{f}\right|_{\left(\widetilde{X^{H}(x)}, X^{>H}(x)\right)}\right)\right]\right) \\
& =\sum_{\substack{\bar{x} \in \operatorname{Is} \Pi(G, X), X^{H}(f(x))=X^{H}(x)}} L^{\mathbb{Z} \operatorname{Aut}(x)\left(\left.\widetilde{f}\right|_{\left(\widetilde{X^{H}(x)}, X^{>H}(x)\right)}\right)} \\
& =\lambda_{G}(f) .
\end{aligned}
$$

So we have a map $\operatorname{tr}_{G(X, f)}:\left(U_{G}^{\mathbb{Z}}(X, f), u_{G}^{\mathbb{Z}}(X, f)\right) \rightarrow\left(\Lambda_{G}(X, f), \lambda_{G}(f)\right)$. By Proposition 7.4.4, we can state: The collection of the $\operatorname{tr}_{G(X, f)}$ is a natural transformation from $\left(U^{\mathbb{Z}}, u^{\mathbb{Z}}\right)$ to $(\Lambda, \lambda)$. The pair $\left(U^{\mathbb{Z}}, u^{\mathbb{Z}}\right)$ is a functorial equivariant Lefschetz invariant on the family of categories $G-\mathcal{C} W_{\mathrm{fp}}$ for discrete groups $G$ and thus satisfies the properties listed in Definition 3.5.1. Since the collection of the $\operatorname{tr}_{G(X, f)}$ is a family of natural transformations of functors from $\operatorname{End}\left(G-\mathcal{C} W_{\mathrm{fp}}\right)$ to $\mathcal{A} b$, for discrete groups $G$, the pair $(\Lambda, \lambda)$ inherits all structure from $\left(U^{\mathbb{Z}}, u^{\mathbb{Z}}\right)$ : It is also a functorial equivariant Lefschetz invariant on the family of categories $G-\mathcal{C} W_{\mathrm{fp}}$ for discrete groups $G$.

Theorem 7.5.2. The pair $(\Lambda, \lambda)$ is a functorial equivariant Lefschetz invariant on the family of categories $G-\mathcal{C} W_{\mathrm{fp}}$ for discrete groups $G$. It consists of a family $\Lambda$ of functors $\Lambda_{G}$ with

$$
\Lambda_{G}: \operatorname{End}\left(G-\mathcal{C} W_{\mathrm{fp}}\right) \rightarrow \mathcal{A} b
$$

that is compatible with the induction structure and a family $\lambda$ of functions $\lambda_{G}:(X, f) \mapsto \lambda_{G}(f) \in \Lambda_{G}(X, f)$ such that the following holds:

\section{Additivity}

For a $G$-pushout with $i_{2}$ a $G$-cofibration

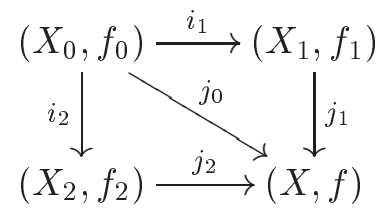

we obtain in $\Lambda_{G}(X, f)$ that

$$
\lambda_{G}(f)=\Lambda_{G}\left(j_{1}\right) \lambda_{G}\left(f_{1}\right)+\Lambda_{G}\left(j_{2}\right) \lambda_{G}\left(f_{2}\right)-\Lambda_{G}\left(j_{0}\right) \lambda_{G}\left(f_{0}\right) .
$$

2. G-Homotopy invariance

If $h_{0}, h_{1}:(X, f) \rightarrow(Y, g)$ are two $G$-maps that are $G$-homotopic in $\operatorname{End}\left(G-\mathcal{C} W_{\mathrm{fp}}\right)$, then

$$
\Lambda_{G}\left(h_{0}\right)=\Lambda_{G}\left(h_{1}\right): \Lambda_{G}(X, f) \rightarrow \Lambda_{G}(Y, g) .
$$


3. Invariance under $G$-homotopy equivalence

If $h:(X, f) \rightarrow(Y, g) \in \operatorname{End}\left(G-\mathcal{C} W_{\mathrm{fp}}\right)$ such that $h: X \rightarrow Y$ is a $G$ homotopy equivalence, then

$$
\begin{aligned}
\Lambda_{G}(h): \Lambda_{G}(X, f) & \cong \Lambda_{G}(Y, g) \\
\lambda_{G}(f) & \mapsto \lambda_{G}(g) .
\end{aligned}
$$

4. Normalization: We have $\lambda_{G}\left(\operatorname{id}_{\emptyset}\right)=0 \in \Lambda_{G}\left(\emptyset, \operatorname{id}_{\emptyset}\right)$.

5. If $\alpha: G \rightarrow K$ is an inclusion of groups, then

$$
\alpha_{*} \lambda_{G}(f)=\lambda_{K}\left(\operatorname{ind}_{\alpha} f\right) \in \Lambda_{K}\left(\operatorname{ind}_{\alpha} X, \operatorname{ind}_{\alpha} f\right) .
$$

Proof. The natural transformation $\operatorname{tr}_{G(X, f)}: U_{G}^{\mathbb{Z}}(X, f) \rightarrow \Lambda_{G}(X, f)$ maps $u_{G}^{\mathbb{Z}}(X, f)$ to $\lambda_{G}(X, f)$. So $\left(\Lambda_{G}(X, f), \lambda_{G}(X, f)\right)$ is a functorial equivariant Lefschetz invariant, which by Definition 3.5.1 has all the properties stated.

Remark 7.5.3. Because of $G$-homotopy invariance and invariance under $G$-homotopy equivalence, analogously to the universal functorial equivariant Lefschetz invariant we can define $\Lambda_{G}(X, f)$ and $\lambda_{G}(X, f)$ for any $G$ equivariant continuous map $f: X \rightarrow X$, we need not restrict ourselves to cellular $G$-maps $f: X \rightarrow X$. See Remark 3.5.3 for details concerning the construction.

Remark 7.5.4. We could also have defined $\lambda_{G}(f)$ by

$$
\lambda_{G}(f)_{\bar{x}}:=\sum_{\bar{\alpha} \in \pi_{1}\left(X^{H}(x), x\right)_{\phi_{x, w_{x}}^{\prime}}} L^{\mathbb{Z} U_{\alpha}}\left(\left.\alpha^{-1} \widetilde{f}\right|_{\left(\widetilde{X^{H}(x)}, X^{>H}(x)\right)}\right) \cdot \bar{\alpha}
$$

if $X^{H}(f(x))=X^{H}(x)$ and by 0 else. Here $\alpha \in \pi_{1}\left(X^{H}(x), x\right)$ and $U_{\alpha}:=$ $\left\{\gamma \in \operatorname{Aut}(x) \mid \phi_{x, w_{x} \alpha^{-1}}(\gamma)=\gamma\right\}$. I.e., $U_{\alpha}$ is the subgroup of $\operatorname{Aut}(x)$ that fixes $\alpha$, while $\operatorname{Aut}(x)$ operates transitively on the $\alpha \in \bar{\alpha}$. For any two $\alpha \in \bar{\alpha}$, the $U_{\alpha}$ 's are conjugated. The map $\alpha^{-1} \widetilde{f}$ is a $U_{\alpha}$-equivariant morphism, whereas as a morphism of $\operatorname{Aut}(x)$-spaces, it is only $\phi$-twisted equivariant. The above expression uses the definitions for non-twisted equivariant maps by Lück and Rosenberg [LR03]. We know that [LR03, Lemma 1.9]

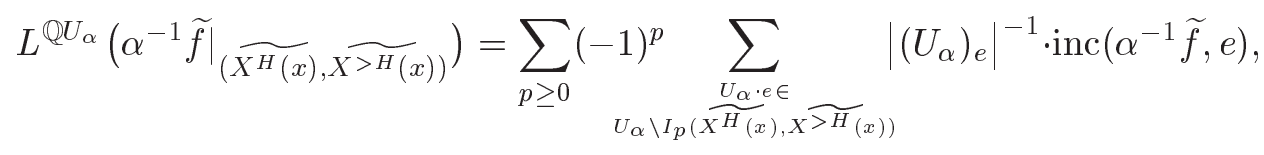

where $\left|\left(U_{\alpha}\right)_{e}\right|=1$ since $U_{\alpha} \leq \operatorname{Aut}(x)$ and $\operatorname{Aut}(x)$ operates freely on $\widetilde{X^{H}(x)} \backslash$ 
$\widetilde{X^{>H}(x)}$, and thus we can use $\mathbb{Z}$ instead of $\mathbb{Q}$ and obtain

$$
\begin{aligned}
& \sum_{\bar{\alpha} \in \mathbb{Z} \pi_{1}\left(X^{H}(x), x\right)_{\phi^{\prime}}} L^{\mathbb{Z} U_{\alpha}}\left(\left.\alpha^{-1} \widetilde{f}\right|_{\left(\widetilde{X^{H}(x)}, X^{>H}(x)\right)}\right) \cdot \bar{\alpha} \\
& =\sum_{p \geq 0}(-1)^{p} \sum_{\bar{\alpha} \in \mathbb{Z} \pi_{1}\left(X^{H}(x), x\right)_{\phi^{\prime}}} \sum_{\substack{U_{\alpha} \cdot e \epsilon \\
U_{\alpha} \backslash I_{p}\left(X^{H}(x), X>H_{(x)}\right)}} \operatorname{inc}\left(\alpha^{-1} \tilde{f}, e\right) \cdot \bar{\alpha} \\
& =\sum_{p \geq 0}(-1)^{p} \sum_{\operatorname{Aut}(x) \backslash\left(\alpha, I_{p}\left(\widetilde{X^{H}(x), X}, X \mathcal{P H}_{(x))}\right)\right.} \operatorname{inc}\left(\alpha^{-1} \widetilde{f}, e\right) \cdot \bar{\alpha}
\end{aligned}
$$

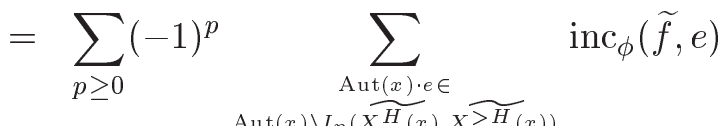

$$
\begin{aligned}
& =L^{\mathbb{Z}} \operatorname{Aut}(x)\left(\widetilde{f^{H}(x)}, \widehat{f>H(x)}\right) .
\end{aligned}
$$

So the two definitions coincide.

\subsection{Relation to the Equivariant Lefschetz Class}

The equivariant analog of the Lefschetz number is $\Lambda^{G}(f)$, the equivariant Lefschetz class [LR03]. We call it $L_{G}(f)$ here to avoid confusion with our notation, and repeat its definition [LR03, Definition 3.6].

Definition 7.6.1. Let $G$ be a discrete group, $X$ a finite proper $G$-CWcomplex and $f: X \rightarrow X$ a $G$-equivariant endomorphism. The equivariant Lefschetz class $L_{G}(f) \in U^{G}(X):=\bigoplus_{\bar{x} \in \operatorname{Is} \Pi(G, X)} \mathbb{Z}$ is defined by

$$
L_{G}(f)_{\bar{x}}= \begin{cases}L^{\mathbb{Z} W H_{x}}\left(f^{H}(x), f^{>H}(x)\right) & \text { if } X^{H}(f(x))=X^{H}(x) \\ 0 & \text { else. }\end{cases}
$$

This is a functorial equivariant Lefschetz invariant [LR03, Lemma 3.7]. The natural transformation mapping the universal functorial equivariant Lefschetz invariant $\left(U_{G}^{\mathbb{Z}}(X, f), u_{G}^{\mathbb{Z}}(X, f)\right)$ to the equivariant Lefschetz class $\left(U^{G}(X), L_{G}(f)\right)$ is given by the trace map $\operatorname{tr}_{G(X, f)}$ followed by an augmentation map $s_{G(X, f)}$ induced by the projection $\pi_{1}\left(X^{H}(x), x\right)_{\phi_{x}^{\prime}} \rightarrow\{1\}$, just like in the non-equivariant case. Define for $\bar{x} \in \operatorname{Is}(G, X)$ with $X^{H}(f(x))=$ $X^{H}(x)$ the augmentation map

$$
\begin{aligned}
s_{\bar{x}}: \Lambda_{G}(X, f)_{\bar{x}}=\mathbb{Z} \pi_{1}\left(X^{H}(x), x\right)_{\phi_{x}^{\prime}} & \rightarrow \mathbb{Z} \\
\sum_{\bar{\alpha}} n_{\bar{\alpha}} \cdot \bar{\alpha} & \mapsto \sum_{\bar{\alpha}} n_{\bar{\alpha}},
\end{aligned}
$$

and set

$$
s_{G(X, f)}:=\left(\bigoplus_{\bar{x} \text { with } X^{H}(f(x))=X^{H}(x)} s_{\bar{x}}\right) \oplus 0: \Lambda_{G}(X, f) \rightarrow U^{G}(X) .
$$


This is a natural transformation of functors. Given a $G$-equivariant map $h:(X, f) \rightarrow\left(Y, f^{\prime}\right)$, for all $\bar{x}$ with $X^{H}(f(x))=X^{H}(x)$ and for all elements $\sum_{\bar{\alpha}} n_{\bar{\alpha}} \cdot \bar{\alpha} \in \Lambda_{G}(X, f)_{\bar{x}}$ we have

$$
\begin{aligned}
s \overline{h(x)} \Lambda_{G}(h)_{\bar{x}} \sum_{\bar{\alpha}} n_{\bar{\alpha}} \cdot \bar{\alpha} & =s \overline{h(x)} \sum_{\bar{\alpha}} n_{\bar{\alpha}} \cdot \overline{\pi_{1}\left(h_{X^{H}}(x), x\right)(\alpha)} \\
& =\sum_{\bar{\alpha}} n_{\bar{\alpha}} \\
& =U^{G}(h)_{\bar{x}} s_{\bar{x}} \sum_{\bar{\alpha}} n_{\bar{\alpha}} \cdot \bar{\alpha}
\end{aligned}
$$

since $U^{G}(h)_{\bar{x}}$ is just the inclusion into the component $U^{G}(Y) \overline{h(x)}$.

Considering the induction structure, given an inclusion $\alpha: G \rightarrow K$, both $\Lambda(\alpha)$ and $U(\alpha)$ are the inclusions into the appropriate component, so

$$
s_{\overline{\operatorname{ind}_{\alpha} x}} \Lambda(\alpha)_{\bar{x}}=U(\alpha)_{\bar{x}} s \bar{x}
$$

So the collection of the $s_{G(X, f)}$ is a family of natural transformations of functors from the categories $\operatorname{End}\left(G-\mathcal{C} W_{\mathrm{fp}}\right)$ to $\mathcal{A} b$, for discrete groups $G$. We have $s_{G(X, f)}\left(\lambda_{G}(f)\right)=L_{G}(f)$ since $s_{\bar{x}}\left(L^{\mathbb{Z} \operatorname{Aut}(x)}\left(\widehat{f^{H}(x)}, \widehat{f^{>H}(x)}\right)\right)=$ $L^{\mathbb{Z} W H_{x}}\left(f^{H}(x), f^{>H}(x)\right)$. We see that $s_{G(X, f)}$ maps $\left(\Lambda_{G}(X, f), \lambda_{G}(f)\right)$ to $\left(U^{G}(X), L_{G}(f)\right)$. It follows that the natural transformation

$$
\tau_{G(X, f)}:=s_{G(X, f)} \circ \operatorname{tr}_{G(X, f)}: U_{G}^{\mathbb{Z}}(X, f) \rightarrow U^{G}(X)
$$

maps $u_{G}(X, f)$ to $L_{G}(f)$. 


\section{Chapter 8}

\section{The Refined Equivariant Lefschetz Fixed Point Theorem}

In this chapter, a generalized local equivariant Lefschetz class is defined, in analogy to the local equivariant Lefschetz class [LR03, Definition 4.6]. This class is given for cocompact proper smooth $G$-manifolds $M$ with $G$ equivariant endomorphisms $f: M \rightarrow M$ such that $\operatorname{Fix}(f) \cap \partial M=\emptyset$ and such that for every $x \in \operatorname{Fix}(f)$ the determinant of the map $\left(\operatorname{id}_{T_{x} M}-T_{x} f\right)$ is different from zero. It is defined in terms of local data around the fixed points of $M$.

Using the generalized local equivariant Lefschetz class, we can prove the refined equivariant Lefschetz fixed point theorem, Theorem 8.2.7, as a generalization of the equivariant Lefschetz fixed point theorem [LR03, Theorem 0.2]. It states that the generalized equivariant Lefschetz invariant and the generalized local equivariant Lefschetz class of such a cocompact proper smooth $G$-manifold $M$ coincide. This shows that the generalized equivariant Lefschetz invariant $\lambda_{G}(f) \in \Lambda_{G}(M, f)$ is given as a sum of fixed point contributions.

\subsection{The Refined Orbifold Lefschetz Fixed Point Theorem}

We need a refinement of the orbifold Lefschetz fixed point theorem [LR03, Theorem 2.1] to smooth manifolds with an action of a discrete group extension with endomorphism. We will apply this refinement to the universal covering spaces appearing in the proof of the refined equivariant Lefschetz fixed point theorem. 
Theorem 8.1.1. Let $G$ be a discrete group extension $1 \rightarrow \pi \rightarrow G \rightarrow W \rightarrow$ 1 with endomorphism $\phi: G \rightarrow G$ as in Definition 7.2.1 such that $\phi_{W}=$ $\operatorname{id}_{W}: W \rightarrow W$. Let $M$ be a connected simply connected cocompact proper $G$ manifold such that $\pi$ operates freely on $M$, and let $f: M \rightarrow M$ be a smooth $\phi$-twisted map. Denote by $\bar{f}: \bar{M} \rightarrow \bar{M}$ the $W$-equivariant map induced on the manifold $\bar{M}:=\pi \backslash M$ by dividing out the $\pi$-action. Suppose that $\operatorname{Fix}(\bar{f}) \cap \partial \bar{M}=\emptyset$ and that for every $x \in \operatorname{Fix}(\bar{f})$ the determinant of the map $\left(\operatorname{id}_{T_{x}} \bar{M}-T_{x} \bar{f}\right)$ is different from zero. Then $W \backslash \operatorname{Fix}(\bar{f})$ is finite, and

$$
L^{\mathbb{Q} G}(f)=\sum_{W \cdot x \in W \backslash \operatorname{Fix}(\bar{f})}\left|W_{x}\right|^{-1} \cdot \operatorname{deg}\left(\left(\operatorname{id}_{T_{x} \bar{M}}-T_{x} \bar{f}\right)^{c}\right) \cdot \overline{\alpha_{x}} .
$$

Here $\left(\operatorname{id}_{T_{x} \bar{M}}-T_{x} \bar{f}\right)^{c}:\left(T_{x} \bar{M}\right)^{c} \rightarrow\left(T_{x} \bar{M}\right)^{c}$ is an endomorphism of the onepoint compactification of $T_{x} \bar{M}$.

Proof. The proof is analogous to the proof of the orbifold Lefschetz fixed point theorem [LR03, Theorem 2.1]. We apply the construction from that proof to the $W$-equivariant map $\bar{f}: \bar{M} \rightarrow \bar{M}$. We obtain a $W$-equivariant map $\overline{f^{\prime}}$ such that $\operatorname{Fix}(\bar{f})=\operatorname{Fix}\left(\overline{f^{\prime}}\right)$, the map $\overline{f^{\prime}}$ is of the desired form around the fixed points and agrees with $\bar{f}$ outside a neighborhood of the fixed points. The desired form is that $\exp _{x, \varepsilon_{1}}^{-1} \circ \bar{f} \circ \exp _{x, \varepsilon_{2}}$ and $\overline{f^{\prime}}$ agree on $D_{\varepsilon} T_{x} \bar{M}$ for some $\varepsilon>0$ and for all $x \in \operatorname{Fix}(\bar{f})$. Here $\exp _{x, \varepsilon}: D_{\varepsilon} T_{x} \bar{M} \stackrel{\sim}{\rightarrow} N_{x, \varepsilon}$ denotes the exponential map [LR03, proof of Theorem 2.1].

We lift this construction to $M$ and the $\phi$-twisted endomorphism $f: M \rightarrow$ $M$ by extending $\phi$-twisted $G$-equivariantly: Let $z$ be a lift of a fixed point $x$, then $f(z)=\alpha_{z} \cdot z$ with $\alpha_{z} \in \pi$, and we set $\left.f^{\prime}\right|_{U_{z}}:=\alpha_{z} \cdot \varphi \overline{f^{\prime}} \varphi^{-1}$ on a neighborhood of $z$ that is isomorphic to a neighborhood of $x$ via the isomorphism $\varphi: U_{z} \stackrel{\sim}{\rightarrow} U_{x}$ coming from the covering map. Around another lift $\beta \cdot z$, we set $\left.f^{\prime}\right|_{U_{\beta z}}:=\left.\phi(\beta) \cdot f^{\prime}\right|_{U_{z}} \cdot \beta^{-1}$ on a neighborhood of $\beta z$. (Note that $\alpha_{\beta z}=\phi(\beta) \cdot \alpha_{z} \cdot \beta^{-1}$.) We obtain a $\phi$-twisted map $f^{\prime}: M \rightarrow M$ such that $\operatorname{Fix}(f)=\operatorname{Fix}\left(f^{\prime}\right)$, the map $f^{\prime}$ is of the desired form around the orbits of the fixed points, and it agrees with $f$ outside a neighborhood of the orbits of the fixed points.

Analogously, we lift all further constructions such as the $W$-equivariant triangulations $K^{\prime}(\bar{M}), K^{\prime \prime}(\bar{M})$ and the $W$-homotopy $\bar{h}$ from $\bar{f}$ to $\overline{h_{1}}$. The construction of $K^{\prime \prime}(\bar{M})$ can be done such that there is at most one fixed point $x$ of $\overline{h_{1}}$ in each $\bar{e} \in K^{\prime \prime}(\bar{M})$. We denote $\overline{h_{1}}$ again by $\bar{f}$. Then

$$
\operatorname{inc}_{\phi}(f, e)= \begin{cases}\operatorname{inc}(\bar{f}, \bar{e}) \cdot \overline{\alpha_{x}} & \text { if there is a fixed point } x \in \bar{e} \\ 0 & \text { else. }\end{cases}
$$

Here for a basepoint $y$ of $\bar{M}$, a path $v_{y}$ from $y$ to $\bar{f}(y)$ and a morphism $(\sigma,[t]): y \rightarrow x$ from $y$ to $x$ we set $\overline{\alpha_{x}}:=\overline{v_{y} f\left(t^{-1}\right) t}$. The direction of $v_{y}$ corresponds to our usual convention that $w_{y}=\left(\mathrm{id},\left[v_{y}\right]\right) \in \operatorname{Mor}(\bar{f}(y), y)$. Note 
that $\overline{\alpha_{x}}$ does not depend on the choice of $(\sigma,[t])$ because of Lemma 7.1.5. We see that $\left|G_{e}\right|=\left|W_{\bar{e}}\right|$ because $\pi$ operates freely. So using the construction of the proof of the orbifold Lefschetz fixed point theorem [LR03] applied to the $W$-equivariant map $\bar{f}$, where $\left\{x_{1}, \ldots, x_{k}\right\}$ is a complete set of representatives of $W$-orbits of fixed points of $\bar{f}$, we obtain

$$
\begin{aligned}
L^{\mathbb{Q} G}(f) & =L^{\mathbb{Q} G}\left(h_{1}\right) \\
& =\sum_{p \geq 0}(-1)^{p} \sum_{G \cdot e \in G \backslash I_{p}\left(K^{\prime \prime}(M)\right)}\left|G_{e}\right|^{-1} \operatorname{inc}_{\phi}(f, e) \\
& =\sum_{p \geq 0}(-1)^{p} \sum_{G \cdot e \in G \backslash I_{p}\left(K^{\prime \prime}(M)\right)}\left|W_{\bar{e}}\right|^{-1} \operatorname{inc}(\bar{f}, \bar{e}) \cdot \overline{\alpha_{x_{\bar{e}}}} \\
& =\sum_{p \geq 0}(-1)^{p} \sum_{W \cdot \bar{e} \in W \backslash I_{p}\left(K^{\prime \prime}(\bar{M})\right)}\left|W_{\bar{e}}\right|^{-1} \operatorname{inc}(\bar{f}, \bar{e}) \cdot \overline{\alpha_{x_{\bar{e}}}} \\
& =\sum_{i=1}^{k}\left|W_{x_{i}}\right|^{-1} \operatorname{deg}\left(\left(\operatorname{id}-T_{x_{i}} \bar{f}\right)^{c}\right) \cdot \overline{\alpha_{x_{i}}} \\
& =\sum_{W \cdot x \in W \backslash \operatorname{Fix}(\bar{f})}\left|W_{x}\right|^{-1} \cdot \operatorname{deg}\left(\left(\mathrm{id}-T_{x} \bar{f}\right)^{c}\right) \cdot \overline{\alpha_{x}}
\end{aligned}
$$

where the second to last equality uses the calculation done in the proof of the orbifold Lefschetz fixed point theorem [LR03, proof of Theorem 2.1].

\subsection{The Local Equivariant Lefschetz Class}

In this section, we introduce the generalized local equivariant Lefschetz class $\lambda_{G}^{l o c}(f)$ in terms of fixed point data. It is a slight refinement of the local equivariant Lefschetz class $\Lambda_{\text {loc }}^{G}(f)$ [LR03, Definition 4.6]. We briefly assemble the notation that we will need in the sequel [LR03, Chapter 4].

Let $K$ be a finite group. The Burnside ring $A(K)$ of $K$ is defined to be the Grothendieck ring of finite $K$-sets $S$ with the additive structure induced by disjoint union and the multiplicative structure induced by the Cartesian product. Additively, $A(K)=U^{K}(*):=\bigoplus_{(H) \in \operatorname{consub}(K)} \mathbb{Z}$, where $*$ denotes the one-point space. Let $Z$ be a finite $K$-CW-complex and let $\psi: Z \rightarrow Z$ be a $K$-equivariant endomorphism. Then the equivariant Lefschetz class with values in the Burnside ring of $\psi$ is defined to be

$$
\Lambda_{0}^{K}(\psi):=\sum_{(H) \in \operatorname{consub}(K)} L^{W H}\left(\psi^{H}, \psi^{>H}\right) \cdot[K / H] \in A(K)=U^{K}(*) .
$$

We call the injective ring homomorphism

$$
\operatorname{ch}_{0}^{K}: A(K) \rightarrow \bigoplus_{(H) \in \operatorname{consub}(K)} \mathbb{Z}, S \mapsto\left\{\left|S^{H}\right|\right\}_{(H) \in \operatorname{consub}(K)}
$$


the character map. The equivariant Lefschetz class is characterized by [LL89, Theorem 2.19] [Lüc88, Lemma 3]

$$
\operatorname{ch}_{0}^{K}\left(\Lambda_{0}^{K}(\psi)\right)=\left\{L^{\mathbb{Z}[\{1\}]}\left(\psi^{H}\right)\right\}_{(H) \in \operatorname{consub}(K)} .
$$

The projection pr: $Z \rightarrow *$ induces a map $U^{K}(\mathrm{pr}): U^{K}(Z) \rightarrow U^{K}(*)$ which sends the equivariant Lefschetz class $L_{G}(\psi)$ (see Definition 7.6.1) to $\Lambda_{0}^{K}(\psi)$.

Let $V$ be a finite-dimensional $K$-representation and let $\psi: V^{c} \rightarrow V^{c}$ be a $K$-endomorphism of the one-point compactification $V^{c}$. Define the equivariant degree of $\psi$ to be

$$
\operatorname{Deg}_{0}^{K}(\psi):=\left(\Lambda_{0}^{K}(\psi)-1\right)\left(\Lambda_{0}^{K}\left(\operatorname{id}_{V^{c}}\right)-1\right) \in A(K)=U^{K}(*) .
$$

We know that $\operatorname{Deg}_{0}^{K}(\psi)$ is uniquely characterized by the property that

$$
\operatorname{ch}_{0}^{K}\left(\operatorname{Deg}_{0}^{K}(\psi)\right)=\left\{\operatorname{deg}\left(\psi^{H}\right)\right\}_{(H) \in \operatorname{consub}(G)} .
$$

Definition 8.2.1. Let $G$ be a discrete group, let $M$ be a cocompact smooth proper $G$-manifold and let $f: M \rightarrow M$ be a $G$-equivariant endomorphism such that $\operatorname{Fix}(f) \cap \partial M=\emptyset$ and such that for every $x \in \operatorname{Fix}(f)$ the determinant of the map $\left(\operatorname{id}_{T_{x} M}-T_{x} f\right)$ is different from zero. Then $G \backslash \operatorname{Fix}(f)$ is finite. We define the generalized local equivariant Lefschetz class to be

$$
\begin{aligned}
\lambda_{G}^{l o c}(f) & :=\sum_{G x \in G \backslash \operatorname{Fix}(f)} \Lambda_{G}(x, f) \circ \operatorname{ind}_{G_{x} \subseteq G}\left(\operatorname{Deg}_{0}^{G_{x}}\left(\left(\operatorname{id}_{T_{x} M}-T_{x} f\right)^{c}\right)\right) \\
& \in \Lambda_{G}(M, f) .
\end{aligned}
$$

Here we have defined

$$
\begin{aligned}
& \Lambda_{G}(x, f): \quad U^{G}\left(G / G_{x}\right) \rightarrow \Lambda_{G}(M, f) \\
& {\left[\tau: G / L \rightarrow G / G_{x}\right] \mapsto \overline{1_{x \circ \tau}} \cdot[x \circ \tau: G / L \rightarrow M],}
\end{aligned}
$$

with $\overline{1_{x \circ \tau}} \in \mathbb{Z} \pi_{1}\left(M^{L}(x \circ \tau), x \circ \tau\right)_{\phi_{x \circ \tau, \text { sst } x \circ \tau^{\prime}}}$.

If we choose $z \cong x \circ \tau: G / L \rightarrow M$ as a fixed representative of the isomorphism class, then the map $\Lambda_{G}(x, f)$ becomes

$$
\begin{aligned}
\Lambda_{G}(x, f): U^{G}\left(G / G_{x}\right) & \rightarrow \Lambda_{G}(M, f) \\
{\left[\tau: G / L \rightarrow G / G_{x}\right] } & \mapsto \overline{\alpha_{x \circ \tau}} \cdot[z],
\end{aligned}
$$

where $\overline{\alpha_{x \circ \tau}}=(\sigma,[t])_{w_{z}, \operatorname{cst}_{x \circ \tau}}^{*}\left(\overline{1_{x \circ \tau}}\right)=\overline{v_{x} f\left(t^{-1}\right) \sigma^{*}\left(1_{x \circ \tau}\right) t}=\overline{v_{x} f\left(t^{-1}\right) t}$ for an isomorphism $(\sigma,[t]) \in \operatorname{Mor}(z, x \circ \tau)$ and a path $w_{z}=\left(\mathrm{id},\left[v_{z}\right]\right) \in \operatorname{Mor}(f(z), z)$. The difference to the local equivariant Lefschetz class $\Lambda_{l o c}^{G}(f) \in U^{G}(M)$ defined by Lück and Rosenberg [LR03, Definition 4.6] is that $\Lambda_{G}(M, f)$ is a larger group than $U^{G}(M)$ which not only remembers the component of the fixed point $x$, but also the appropriate $\bar{\alpha}$. We also need to generalize the character map $\operatorname{ch}^{G}(X): U^{G}(X) \rightarrow \bigoplus_{\text {Is } \Pi(G, X)} \mathbb{Q}$ [LR03, Equation 5.1]. 
Definition 8.2.2. We define the character map

$$
\operatorname{ch}_{G}(X, f): \Lambda_{G}(X, f) \rightarrow \bigoplus_{\bar{y} \in \operatorname{Is} \Pi(G, X)} \mathbb{Q} \Pi(G, X)_{\phi, \bar{y}},
$$

where $\mathbb{Q} \Pi(G, X)_{\phi, \bar{y}}=\mathbb{Z} \Pi(G, X)_{\phi, \bar{y}} \otimes_{\mathbb{Z}} \mathbb{Q}$, by

$$
\begin{aligned}
& \operatorname{ch}_{G}(X, f)\left(\left(\sum_{\bar{\alpha}} n_{\bar{\alpha}} \cdot \bar{\alpha}\right) \cdot \bar{x}\right)_{\bar{y}} \\
& :=\sum_{\substack{\operatorname{Aut}(y) \cdot(\sigma,[t]) \in \\
\operatorname{Aut}(y) \backslash \operatorname{Mor}(y, x)}}\left|(\operatorname{Aut}(y))_{(\sigma,[t])}\right|^{-1} \cdot(\sigma,[t])^{*}\left(\sum_{\bar{\alpha}} n_{\bar{\alpha}} \cdot \bar{\alpha}\right) .
\end{aligned}
$$

Here it is important that we defined $(\sigma,[t])^{*}$ for all maps, not only for isomorphisms. The character map $\mathrm{ch}^{G}(X)$ is injective [LR03, Lemma 5.3], and so is the character map $\operatorname{ch}_{G}(X, f)$ :

Lemma 8.2.3. The character map $\operatorname{ch}_{G}(X, f)$ is injective.

Proof. Let $u=\sum_{i=1}^{k} a_{i} \cdot \overline{x_{i}} \in \Lambda_{G}(X, f)$, where $a_{i} \in \Lambda_{G}(X, f)_{\overline{x_{i}}}$, with $\operatorname{ch}_{G}(X, f)(u)=0$. Let the $x_{i}$ be ordered in accordance with the partial ordering on $\Pi(G, X)$ given by $\bar{x} \leq \bar{y} \Leftrightarrow \operatorname{Mor}(x, y) \neq 0$, so $x_{i} \leq x_{j} \Rightarrow i \leq j$. Suppose without loss of generality that $a_{k} \neq 0$. If $\operatorname{ch}_{G}(X, f)\left(\overline{x_{i}}\right)_{\overline{x_{k}}} \neq 0$, then $\operatorname{Mor}\left(x_{k}, x_{i}\right) \neq 0$, which implies $x_{k} \leq x_{i}$ and thus $x_{k}=x_{i}$. Since $\operatorname{ch}_{G}(X, f)\left(a_{k} \cdot \overline{x_{k}}\right)_{\overline{x_{k}}}=a_{k}$, we obtain $0=\operatorname{ch}^{G}(X, f)(u)=a_{k}$, a contradiction.

The character map $\operatorname{ch}_{G}(X, f)$ is $G$-homotopy invariant in the following sense: If $f \simeq_{G} f^{\prime}$, with a $G$-homotopy $H: X \times I \rightarrow X$ such that $H_{0}=f$ and $H_{1}=f^{\prime}$, then by invariance under $G$-homotopy equivalence we have an isomorphism $\Lambda_{G}\left(i_{1}\right)^{-1} \Lambda_{G}\left(i_{0}\right): \Lambda_{G}(X, f) \stackrel{\sim}{\rightarrow} \Lambda_{G}\left(X, f^{\prime}\right)$ which sends $\lambda_{G}(f)$ to $\lambda_{G}\left(f^{\prime}\right)$. An isomorphism $i_{0 *}: \mathbb{Q} \Pi(G, X)_{\phi, \bar{y}} \rightarrow \mathbb{Q} \Pi(G, X \times I)_{\Phi, \overline{i_{0}(y)}}$ is induced by the inclusion $i_{0}$, and analogously $i_{1}$ induces an isomorphism. We have $\operatorname{ch}_{G}\left(X, f^{\prime}\right) \Lambda_{G}\left(i_{1}\right)^{-1} \Lambda_{G}\left(i_{0}\right)=\left(i_{1 *}\right)^{-1} i_{0 *} \operatorname{ch}_{G}(X, f)$.

We have now set the stage for the main part of this chapter. We calculate the value of $\operatorname{ch}_{G}(X, f)$ on the generalized equivariant Lefschetz invariant $\lambda_{G}(f)$ and on the generalized local Lefschetz class $\lambda_{G}^{l o c}(f)$. This is in analogy to [LR03, Lemma 5.4 and Lemma 5.9].

Lemma 8.2.4. Let $f: X \rightarrow X$ be a G-equivariant endomorphism of a finite proper $G$-CW-complex $X$. Let $\bar{y}$ be an isomorphism class of objects $y: G / K \rightarrow X$ in $\Pi(G, X)$. Then

$$
\operatorname{ch}_{G}(X, f)\left(\lambda_{G}(f)\right)_{\bar{y}}=L^{\mathbb{Q} \operatorname{Aut}(y)}\left(\widetilde{f^{K}(y)}\right) .
$$


Proof. We first consider the case $X^{K}(f(y))=X^{K}(y)$. We can write $X_{p}$, the $p$-th skeleton of $X$, as a $G$-pushout

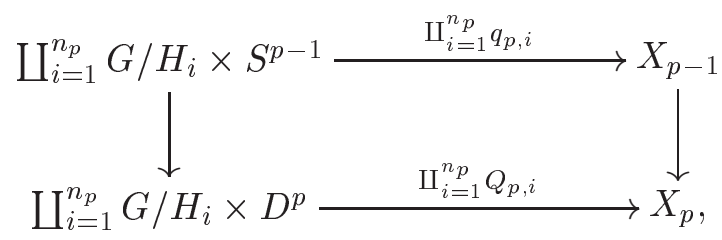

where $n_{p} \geq 0$ and where the $H_{i} \leq G$ are finite subgroups. We set $x_{p, i}:=$ $\left.Q_{p, i}\right|_{G / H_{i} \times\{0\}}: G / H_{i} \rightarrow X$ for $i \in I_{p}$.

The $G$-CW-structure on $X$ induces an Aut $(y)$-CW-structure on $\widetilde{X^{K}(y)}$. We obtain a pushout diagram of $\operatorname{Aut}(y)$-spaces

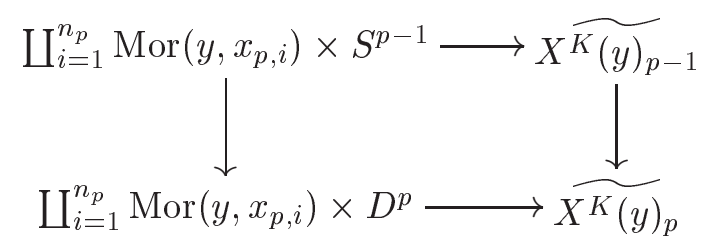

(If $p-1 \leq 1$, then use the appropriate cover, the one corresponding to $\pi_{1}\left(X^{K}(y), y\right)$.) Then the $p$-cells of $\widetilde{X^{K}(y)}$ are $\coprod_{i=1}^{n_{p}} \operatorname{Mor}\left(y, x_{p, i}\right) \times D^{p}$, we call them $e_{(\sigma,[t]), p, i}:=(\sigma,[t]) \times D^{\circ}$, where $(\sigma,[t]) \in \operatorname{Mor}\left(y, x_{p, i}\right)$. The $\operatorname{Aut}(y)$ orbit of the cell $e_{(\sigma,[t]), p, i}$ corresponds to the Aut $(y)$-orbit of $(\sigma,[t])$, so we conclude from Lemma 7.3.5 that

$$
\begin{aligned}
L^{\mathbb{Q}} \operatorname{Aut}(y) & \left(\widetilde{f^{K}(y)}\right) \\
& =\sum_{p \geq 0}(-1)^{p} \sum_{\substack{\operatorname{Aut}(y) \cdot e \in \\
\operatorname{Aut}(y) \backslash y_{p}\left(X^{K}(y)\right)}}\left|\operatorname{Aut}(y)_{e}\right|^{-1} \cdot \operatorname{inc}_{\phi_{y, w_{y}}}\left(\widetilde{f^{K}(y)}, e\right) \\
& =\sum_{p \geq 0}(-1)^{p} \sum_{i=1}^{n_{p}} \sum_{\substack{\operatorname{Aut}(y) \cdot(\sigma,[t]) \in \\
\operatorname{Aut}(y) \backslash \operatorname{Mor}\left(y, x_{p, i}\right)}}\left|\operatorname{Aut}(y)_{(\sigma,[t])}\right|^{-1} \cdot \operatorname{inc}_{\phi_{y, w_{y}}}\left(\widetilde{f^{K}(y)}, e_{(\sigma,[t]), p, i}\right) .
\end{aligned}
$$

Analogously, we have for any $x: G / H \rightarrow X$ a pushout diagram

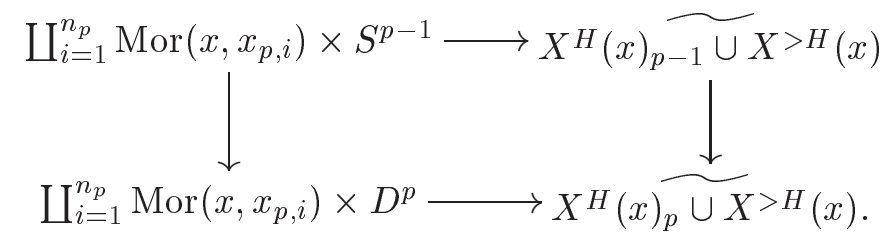


Lemma 7.3.5 yields

$$
\begin{aligned}
& L^{\mathbb{Z} \operatorname{Aut}(x)}\left(\widetilde{f^{H}(x)}, \widetilde{f>H}(x)\right) \\
& =\sum_{p \geq 0}(-1)^{p} \sum_{\substack{\text { Aut }(x) \cdot(\tau,[s]) \in \\
\operatorname{Aut}(x) \backslash \operatorname{Mor}\left(x, x_{p, i}\right)}} \operatorname{inc}_{\phi_{x, w_{x}}}\left(\widetilde{f^{H}(x)}, e_{(\tau,[s]), p, i}\right) \\
& =\sum_{p \geq 0}(-1)^{p} \sum_{\substack{i=1 \\
x_{p, i}=\bar{x}}}^{n_{p}}(\tau,[s])^{*} \operatorname{inc}_{\phi_{x_{p, i}, \operatorname{cst}_{x_{p, i}}}}\left(\widetilde{f^{H}\left(x_{p, i}\right)}, e_{p, i}\right) .
\end{aligned}
$$

So Definition 7.5.1 of the generalized equivariant Lefschetz invariant $\lambda_{G}(f)$ and the above formula yield, inserted into the formula for $\operatorname{ch}_{G}(X, f)$,

$$
\begin{aligned}
& \operatorname{ch}_{G}(X, f)\left(\lambda_{G}(f)\right)_{\bar{y}} \\
& =\sum_{\bar{x}, \operatorname{Mor}(y, x) \neq 0} \sum_{\substack{\operatorname{Aut}(y) \cdot(\sigma,[t]) \in \\
\operatorname{Aut}(y) \backslash \operatorname{Mor}(y, x)}}\left|(\operatorname{Aut}(y))_{(\sigma,[t])}\right|^{-1} \cdot(\sigma,[t])^{*}\left(\lambda_{G}(f)_{x}\right) \\
& =\sum_{\bar{x}, \operatorname{Mor}(y, x) \neq 0} \sum_{\substack{\operatorname{Aut}(y) \cdot(\sigma,[t]] \in \\
\operatorname{Aut}(y) \backslash \operatorname{Mor}(y, x)}}\left|(\operatorname{Aut}(y))_{(\sigma,[t])}\right|^{-1} . \\
& (\sigma,[t])^{*}\left(\sum_{p \geq 0}(-1)^{p} \sum_{\overline{x_{p, i}}=\bar{x}, i=1}^{n_{p}}(\tau,[s])^{*} \operatorname{inc}_{\phi_{x_{p, i}, \operatorname{cst}_{x_{p}, i}}}\left(\widetilde{f^{H}\left(x_{p, i}\right)}, e_{p, i}\right)\right) \\
& =\sum_{p \geq 0}(-1)^{p} \sum_{i=1}^{n_{p}} \sum_{\substack{\operatorname{Aut}(y) \cdot\left(\sigma^{\prime},\left[t^{\prime}\right]\right) \in \\
\operatorname{Aut}(y) \backslash\left(\operatorname{Mor}\left(y, x_{p, i}\right)\right.}}\left|(\operatorname{Aut}(y))_{(\sigma,[t])}\right|^{-1} . \\
& \left(\sigma^{\prime},\left[t^{\prime}\right]\right)^{*} \operatorname{inc}_{\phi_{x_{p, i}, \operatorname{cst}_{x_{p, i}}}}\left(\widetilde{f^{H}\left(x_{p, i}\right)}, e_{p, i}\right),
\end{aligned}
$$

where $\left(\sigma^{\prime},\left[t^{\prime}\right]\right)=(\tau,[s]) \circ(\sigma,[t])$. If we can show that

$$
\left(\sigma^{\prime},\left[t^{\prime}\right]\right)^{*} \operatorname{inc}_{\phi_{x_{p, i}, \operatorname{cst}_{p, i}}}\left(\widetilde{f^{H}\left(x_{p, i}\right)}, e_{p, i}\right)=\operatorname{inc}_{\phi_{y, w_{y}}}\left(\widetilde{f^{K}(y)}, e_{\left(\sigma^{\prime},\left[t^{\prime}\right]\right), p, i}\right),
$$

our lemma is proved. We will state and prove this separately in Lemma 8.2.5 immediately after finishing this proof.

Now we consider the case that $X^{K}(f(y)) \neq X^{K}(y)$. By the observation following Definition 7.1.7, this implies that $X^{H}(f(x)) \neq X^{H}(x)$ for all $\bar{x}$ with $\operatorname{Mor}(y, x) \neq \emptyset$, so $\lambda_{G}(f)_{\bar{x}}=0$ for all $\bar{x}$ with $\operatorname{Mor}(y, x) \neq \emptyset$. This implies that $\operatorname{ch}_{G}(X, f)\left(\lambda_{G}(f)\right)_{\bar{y}}=0$.

Lemma 8.2.5. Let $(\sigma,[t]) \in \operatorname{Mor}_{\Pi(G, X)}(y, x)$, where $y: G / K \rightarrow X$ and where $x: G / H \rightarrow X$ is a fixed point of $f$, i.e., $f(x)=x$. Then for the cell $e \in I_{p}\left(\widehat{X^{H}(x)}, \widehat{X^{>H}(x)}\right)$ which contains $x$, we have

$$
(\sigma,[t])^{*} \operatorname{inc}_{\phi_{x, \mathrm{cst}_{x}}}\left(\widetilde{f^{H}(x)}, e\right)=\operatorname{inc}_{\phi_{y, w_{y}}}\left(\widetilde{f^{K}(y)}, e_{(\sigma,[t])}\right) .
$$


Proof. We know that $(\sigma,[t])=\left(\sigma,\left[\operatorname{cst}_{x}\right]\right) \circ(\mathrm{id},[t])$, where $(\mathrm{id},[t])$ is an isomorphism. For these we know compatibility by Lemma 7.4.1, so it remains to prove that

$$
\left(\sigma,\left[\operatorname{cst}_{x}\right]\right)^{*} \operatorname{inc}_{\phi_{x, \operatorname{cst}_{x}}}(\widetilde{f H(x)}, e)=\operatorname{inc}_{\phi_{x \circ \sigma, \operatorname{cst}_{x \circ \sigma}}}\left(\widetilde{f^{K(x \circ \sigma)}}, e_{\left(\sigma,\left[\operatorname{cst}_{x}\right]\right)}\right) .
$$

We have $\sigma: G / K \rightarrow G / H, g^{\prime} K \mapsto g^{\prime} g_{\sigma} H$, so $\sigma(1 K)=g_{\sigma} H$. By replacing $x$ with $g_{\sigma} x: G / H^{\prime} \rightarrow X$, where $H^{\prime}=g_{\sigma} H g_{\sigma}^{-1}$, we can suppose without loss of generality that $\sigma(1 K)=(1 H)$. We know that

$$
\begin{aligned}
\left(\sigma,\left[\operatorname{cst}_{x}\right]\right)^{*}: \quad \mathbb{Z} \pi_{1}\left(X^{H}(x), x\right)_{\phi_{x, \operatorname{cst}_{x}}^{\prime}} & \rightarrow \mathbb{Z} \pi_{1}\left(X^{K}(x \circ \sigma), x \circ \sigma\right)_{\phi_{x \circ \sigma, \operatorname{cst}_{x \circ \sigma}^{\prime}}} \\
\bar{\alpha} & \mapsto \overline{\sigma^{*}(\alpha)}
\end{aligned}
$$

corresponds to the morphism which is induced by the inclusion $i: X^{H}(x) \rightarrow$ $X^{K}(x \circ \sigma)$ on $\pi_{1}\left(X^{H}(x), x\right)$.

Denoting by $A$ the pullback of the diagram

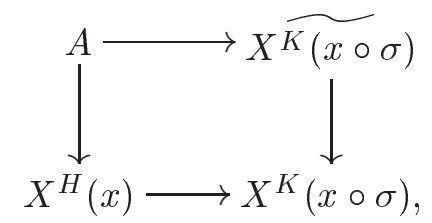

i.e., the covering of $X^{H}(x)$ corresponding to $\pi_{1}\left(X^{K}(x \circ \sigma), x \circ \sigma\right)$, we know that

$$
A=\coprod_{\pi_{1}\left(X^{K}(x \circ \sigma), x \circ \sigma\right) / i_{*}\left(\pi_{1}\left(X^{H}(x), x\right)\right)} \widetilde{X^{H}(x)} / \operatorname{ker}\left(i_{*}\right) .
$$

What is $\operatorname{inc}_{\phi_{x \circ \sigma, \operatorname{cst}_{x \circ \sigma}}}\left(\widetilde{f^{K}(x \circ \sigma)}, e_{\left(\sigma,\left[\operatorname{cst}_{x}\right]\right)}\right)$ ? It tells us to how many, and to which, cells in the $\pi_{1}\left(X^{K(x \circ \sigma)}, x \circ \sigma\right)$-orbit of $e_{\left(\sigma,\left[\operatorname{cst}_{x}\right]\right)}$ the cell $e_{\left(\sigma,\left[\operatorname{cst}_{x}\right]\right)}$ goes (modulo the equivalence relation). We have chosen $\widetilde{f^{K}(x \circ \sigma)}$ such that $x \circ \sigma$ goes to $x \circ \sigma$, and since the cell $e$ is connected and contains $x$, its image is connected and contains $x \circ \sigma$. We also know that its image must lie in $A$ because the cell is fixed by $H$ and by $G$-equivariance of $f$ its image must be fixed by $H$ again. So its lift must lie over $X^{H}(x)$. Since this lift is connected, it must already lie in one of the components $\widehat{X^{H}(x)} / \operatorname{ker}\left(i_{*}\right)$. The cells in $\widetilde{X^{H}(x)} / \operatorname{ker}\left(i_{*}\right)$ to which $e$ goes are those to which $e$ goes in $\widehat{X^{H}(x)}$, projected down. After going over to the equivalence relation, this is nothing but $\operatorname{inc}_{\phi_{x, \text { cst } x}}\left(\widehat{f^{H}(x)}, e\right)$, projected down by $\left(\sigma,\left[c s t_{x}\right]\right)^{*}$. So we have

$$
\left(\sigma,\left[\operatorname{cst}_{x}\right]\right)^{*} \operatorname{inc}_{\phi_{x, \operatorname{cst}_{x}}}\left(\widetilde{f^{H}(x)}, e\right)=\operatorname{inc}_{\phi_{x \circ \sigma, \operatorname{cst}_{x \circ \sigma}}}\left(\widetilde{f^{K}(x \circ \sigma)}, e_{\left(\sigma,\left[\operatorname{cst}_{x}\right]\right)}\right) .
$$


Lemma 8.2.6. Let $G$ be a discrete group and let $M$ be a cocompact smooth proper $G$-manifold. Let $f: M \rightarrow M$ be a smooth $G$-equivariant map. Suppose that $\operatorname{Fix}(f) \cap \partial M=\emptyset$ and that for any $x \in \operatorname{Fix}(f)$ the determinant $\operatorname{det}\left(\mathrm{id}_{T_{x}}-T_{x} f\right)$ is different from zero. Then $G \backslash \operatorname{Fix}(f)$ is finite. Let $y: G / K \rightarrow M$ be an object in $\Pi(G, M)$. Then $W K_{y} \backslash \operatorname{Fix}\left(\left.f\right|_{M^{K}(y)}\right)$ is finite and we get

$$
\begin{aligned}
& \operatorname{ch}_{G}(M, f)\left(\lambda_{G}^{l o c}(f)\right)_{\bar{y}} \\
& =\sum_{\substack{W K_{y} \cdot x \in \\
W K_{y} \backslash \operatorname{Fix}\left(\left.f\right|_{M^{K}(y)}\right)}}\left|\left(W K_{y}\right)_{x}\right|^{-1} \operatorname{deg}\left(\left(\operatorname{id}_{T_{x} M^{K}(y)}-T_{x}\left(\left.f\right|_{M^{K}(y)}\right)\right)^{c}\right) \cdot \overline{\alpha_{x}},
\end{aligned}
$$

where $\overline{\alpha_{x}}=\overline{v_{y} f\left(t^{-1}\right) t} \in \pi_{1}\left(X^{K}(y), y\right)_{\phi^{\prime}}$ for $(\sigma,[t]) \in \operatorname{Mor}(y, x)$ and $w_{y}=$ (id, $\left.\left[v_{y}\right]\right) \in \operatorname{Mor}(f(y), y)$.

Proof. The set $G \backslash \operatorname{Fix}(f)$ is finite since $M$ is cocompact and the fixed points are isolated. Analogously, $G \backslash G M^{K}(y)=W K_{y} \backslash M^{K}(y)$ is compact with isolated fixed points, so $W K_{y} \backslash \operatorname{Fix}\left(\left.f\right|_{M^{K}(y)}\right)$ is finite.

Let $x: G / G_{x} \rightarrow M$ be a fixed point of $f$. We first show that for each $u \in U^{G_{x}}(*)$ we have

$$
\begin{aligned}
& \operatorname{ch}_{G}(M, f)_{\bar{y}} \circ \Lambda_{G}(x, f) \circ \operatorname{ind}_{G_{x} \subseteq G}(u) \\
& \quad=\sum_{\substack{\operatorname{Aut}(y) \cdot(\sigma,[t]] \in \\
\operatorname{Aut}(y) \backslash \operatorname{Mor}(y, x)}}\left|\operatorname{Aut}(y)_{(\sigma,[t])}\right|^{-1} \cdot(\sigma,[t])^{*} \overline{1_{x}} \cdot \operatorname{ch}_{0}^{G_{x}}(u)_{\left(K_{\sigma}\right)},
\end{aligned}
$$

where $\overline{1_{x}} \in \mathbb{Z} \pi_{1}\left(X^{G_{x}}(x), x\right)_{\phi_{x, c s t} t_{x}}$. Here we have $\left(K_{\sigma}\right)=\left(g_{\sigma}^{-1} K g_{\sigma}\right) \in$ $\operatorname{consub}\left(G_{x}\right)$ for $\sigma: G / K \rightarrow G / G_{x}, g K \mapsto g g_{\sigma} G_{x}$. Let $u=\left[G_{x} / L\right] \in U^{G_{x}}(*)$ be a basis element and pr: $G / L \rightarrow G / G_{x}$ the projection. We obtain

$$
\begin{aligned}
& \operatorname{ch}_{G}(M, f)_{\bar{y}} \circ \Lambda_{G}(x, f) \circ \operatorname{ind}_{G_{x} \subseteq G}\left(\left[G_{x} / L\right]\right) \\
& \quad=\operatorname{ch}_{G}(M, f)_{\bar{y}}\left([x \circ \mathrm{pr}: G / L \rightarrow M] \cdot \overline{1_{x \circ \mathrm{pr}}}\right) \\
& =\sum_{\substack{\operatorname{Aut}(y) \cdot(\tau,[t]] \in \\
\operatorname{Aut}(y) \backslash \operatorname{Mor}(y, x \circ \mathrm{pr})}}\left|\operatorname{Aut}(y)_{(\tau,[t])}\right|^{-1}(\tau,[t])^{*} \overline{1_{x \circ \mathrm{pr}}}
\end{aligned}
$$

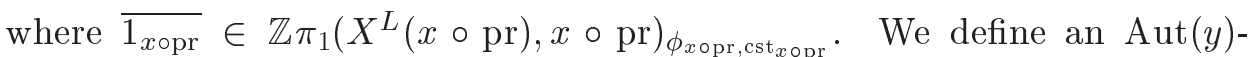
equivariant map $q: \operatorname{Mor}(y, x \circ \operatorname{pr}) \rightarrow \operatorname{Mor}(y, x),(\tau,[t]) \mapsto(\operatorname{pr} \circ \tau,[t])$ and obtain

$$
\begin{aligned}
\operatorname{Mor}(y, x \circ \operatorname{pr}) & =\coprod_{(\sigma,[t]) \in \operatorname{Mor}(y, x)} q^{-1}(\sigma,[t]) \\
& =\coprod_{\substack{\operatorname{Aut}(y) \cdot(\sigma,[t]] \in \\
\operatorname{Aut}(y) \backslash \operatorname{Mor}(y, x)}} \operatorname{Aut}(y) \times_{\operatorname{Aut}(y)_{(\sigma,[t])}} q^{-1}(\sigma,[t]) .
\end{aligned}
$$


The $\left.\operatorname{Aut}(y)_{(\sigma,[t])}\right)^{- \text {set }} q^{-1}(\sigma,[t])$ is a finite disjoint union of orbits

$$
q^{-1}(\sigma,[t])=\coprod_{i \in I(\sigma,[t])} \operatorname{Aut}(y)_{(\sigma,[t])} / A_{i}
$$

thus we have a bijection of $\operatorname{Aut}(y)$-sets

$$
\operatorname{Mor}(y, x \circ \operatorname{pr})=\coprod_{\substack{\operatorname{Aut}(y) \cdot(\sigma,[t]] \in \\ \operatorname{Aut}(y) \backslash \operatorname{Mor}(y, x)}} \coprod_{i \in I(\sigma,[t])} \operatorname{Aut}(y) / A_{i} .
$$

We know for $(\tau,[t]) \in \operatorname{Mor}(y, x \circ \mathrm{pr})$ that $(\tau,[t]) * \overline{1} \overline{1_{x \circ \mathrm{pr}}}=\overline{v_{y} f\left(t^{-1}\right) t}=$ $(\operatorname{pr} \circ \tau,[t]) * \overline{1_{x}}$. An orbit $\operatorname{Aut}(y) / A_{i}$ corresponds to exactly one orbit $\operatorname{Aut}(y)$. $(\tau,[t])$, where $i \in I(\operatorname{pr} \circ \tau,[t])$, so $\left|\operatorname{Aut}(y)_{(\tau,[t])}\right|=\left|A_{i}\right|$, hence

$$
\begin{aligned}
& \sum_{\substack{\operatorname{Aut}(y) \cdot(\tau,[t]) \in \\
\operatorname{Aut}(y) \backslash \operatorname{Mor}(y, x \circ \operatorname{xopr})}}\left|\operatorname{Aut}(y)_{(\tau,[t])}\right|^{-1} \cdot(\tau,[t])^{*} \overline{1_{x \circ \mathrm{prr}}} \\
& =\sum_{\substack{\operatorname{Aut}(y) \cdot(\sigma,[t]) \in \\
\operatorname{Aut}(y) \backslash \operatorname{Mor}(y, x)}} \sum_{i \in I(\sigma,[t])}\left|A_{i}\right|^{-1} \cdot(\sigma,[t])^{*} \overline{1_{x}}
\end{aligned}
$$

We have

$$
\left|q^{-1}(\sigma,[t])\right|=\left|\operatorname{Aut}(y)_{(\sigma,[t])}\right| \cdot \sum_{i \in I(\sigma,[t])}\left|A_{i}\right|^{-1}
$$

So

$$
\begin{aligned}
& \operatorname{ch}_{G}(M, f)_{\bar{y}} \circ \Lambda_{G}(x, f) \circ \operatorname{ind}_{G_{x} \subseteq G}\left(\left[G_{x} / L\right]\right) \\
& \quad=\sum_{\substack{\operatorname{Aut}(y) \cdot(\sigma,[t]) \in \in \\
\operatorname{Aut}(y) \backslash \operatorname{Mor}(y, x)}}\left|\operatorname{Aut}(y)_{(\sigma,[t])}\right|^{-1} \cdot\left|q^{-1}(\sigma,[t])\right| \cdot(\sigma,[t])^{*} \overline{1_{x}}
\end{aligned}
$$

The map $q$ does not change the $[t]$-part, so as in the proof of the corresponding non-generalized result [LR03, Lemma 5.9, Equation 5.14] we have

$$
\left|q^{-1}(\sigma,[t])\right|=\left|G_{x} / L^{g_{\sigma}^{-1} K g_{\sigma}}\right|=\operatorname{ch}_{0}^{G_{x}}\left(\left[G_{x} / L\right]\right)_{\left(K_{\sigma}\right)} .
$$

We have the desired equation for all basis elements $\left[G_{x} / L\right] \in U^{G_{x}}(*)$, thus for all $u \in U^{G_{x}}(*)$. We know that [LR03, Equations 5.16 and 5.17]

$$
\operatorname{ch}_{0}^{G_{x}}\left(\operatorname{Deg}_{0}^{G_{x}}\left(\left(\operatorname{id}_{T_{x} M}-T_{x} f\right)^{c}\right)\right)_{\left(K_{\sigma}\right)}=\operatorname{deg}\left(\left(\operatorname{id}_{T_{g_{\sigma} x} M^{K}(y)}-T_{g_{\sigma} x}\left(\left.f\right|_{M^{K}(y)}\right)\right)^{c}\right) .
$$

We easily observe that

$$
\coprod_{G \backslash \operatorname{Fix}(f)} \operatorname{Aut}(y) \backslash \operatorname{Mor}(y, x) \cong W K_{y} \backslash \operatorname{Fix}\left(\left.f\right|_{M^{K}(y)}\right) .
$$


Under this bijection, $\operatorname{Aut}(y) \cdot(\sigma,[t]) \mapsto W K_{y} \cdot t(0)$, where $t(0)=x \circ \sigma(1 K)=$ $x\left(g_{\sigma} G_{x}\right)=g_{\sigma} x\left(1 G_{x}\right)=g_{\sigma} x$ for $\sigma: G / K \rightarrow G / G_{x}, g K \mapsto g g_{\sigma} G_{x}$. We see that $\left|\operatorname{Aut}(y)_{(\sigma,[t])}\right|=\left|\left(W K_{y}\right)_{g_{\sigma} x}\right|$, so setting $x^{\prime}=g_{\sigma} x$ we obtain

$$
\begin{aligned}
& \operatorname{ch}_{G}(M, f)\left(\lambda_{G}^{\text {loc }}(f)\right)_{\bar{y}} \\
& =\sum_{G \backslash \operatorname{Fix}(f)} \sum_{\substack{\operatorname{Aut}(y) \cdot(\sigma,[t]) \in \\
\operatorname{Aut}(y) \backslash \operatorname{Mor}(y, x)}}\left|\operatorname{Aut}(y)_{(\sigma,[t])}\right|^{-1} . \\
& \operatorname{deg}\left(\left(\operatorname{id}_{T_{g_{\sigma} x} M^{K}(y)}-T_{g_{\sigma} x}\left(\left.f\right|_{M^{K}(y)}\right)\right)^{c}\right) \cdot(\sigma,[t])^{*} \overline{1_{x}} \\
& =\sum_{\substack{W K_{y} \cdot x^{\prime} \in \\
W K_{y} \backslash \operatorname{Fix}\left(\left.f\right|_{M} K^{\prime}(y)\right.}}\left|\left(W K_{y}\right)_{x^{\prime}}\right|^{-1} \cdot \operatorname{deg}\left(\left(\operatorname{id}_{T_{x^{\prime}} M^{K}(y)}-T_{x^{\prime}}\left(\left.f\right|_{M^{K}(y)}\right)\right)^{c}\right) \cdot \overline{\alpha_{x^{\prime}}} \text {, }
\end{aligned}
$$

where $\overline{\alpha_{x^{\prime}}}=\overline{v_{y} f\left(t^{-1}\right) t} \in \pi_{1}\left(X^{K}(y), y\right)_{\phi^{\prime}}$, where $(\sigma,[t]) \in \operatorname{Mor}(y, x)$, so $t$ is a path from $g_{\sigma} x=x^{\prime}$ to $y$.

We have assembled all information necessary for the proof of the refined equivariant Lefschetz fixed point theorem.

Theorem 8.2.7. Let $G$ be a discrete group, let $M$ be a cocompact proper smooth $G$-manifold and let $f: M \rightarrow M$ be a G-equivariant endomorphism such that $\operatorname{Fix}(f) \cap \partial M=\emptyset$ and such that for every $x \in \operatorname{Fix}(f)$ the determinant of the map $\left(\mathrm{id}_{T_{x} M}-T_{x} f\right)$ is different from zero. Then

$$
\lambda_{G}(f)=\lambda_{G}^{l o c}(f) .
$$

Proof. Using Lemma 8.2.4, Theorem 8.1.1 and Lemma 8.2.6 we obtain

$$
\begin{aligned}
\operatorname{ch}_{G}(M, f)\left(\lambda_{G}(f)\right)_{\bar{y}} \\
=L^{\mathbb{Q} \operatorname{Aut}(y)}\left(\widehat{f^{K}(y)}\right) \\
=\sum_{\substack{\left.W K_{y} \cdot x \in \\
W K_{y} \backslash \operatorname{Fix}_{(j \mid}\right|_{M} ^{K}(y)}}\left|\left(W K_{y}\right)_{x}\right|^{-1} \operatorname{deg}\left(\left(\operatorname{id}_{T_{x} M^{K}(y)}-T_{x}\left(\left.f\right|_{M^{K}(y)}\right)\right)^{c}\right) \cdot \overline{\alpha_{x}} \\
=\operatorname{ch}_{G}(M, f)\left(\lambda_{G}^{l o c}(f)\right)_{\bar{y}}
\end{aligned}
$$

for all $\bar{y} \in \operatorname{Is} \Pi(G, X)$. By injectivity of $\operatorname{ch}_{G}(M, f)$, shown in Lemma 8.2.3, we obtain the refined equivariant Lefschetz fixed point theorem.

From the fact that the units of the Burnside ring $A(K)$ are only $\{1,-1\}$ if $K$ is a finite group of odd order [tD79, Proposition 1.5.1], we obtain in analogy to [LR03, Example 4.7] the following example.

Example 8.2.8. Let $G$ be a discrete group and let $M$ be a cocompact proper smooth $G$-manifold. Suppose that all isotropy groups $G_{x}$ of points $x \in M$ 
are of odd order. Let $f: M \rightarrow M$ be a smooth $G$-equivariant map such that $\operatorname{Fix}(f) \cap \partial M=\emptyset$ and such that for every $x \in \operatorname{Fix}(f)$ the determinant of the $\operatorname{map}\left(\operatorname{id}_{T_{x} M}-T_{x} f\right)$ is different from zero. Then

$$
\begin{aligned}
\lambda_{G}^{l o c}(f) & =\sum_{\substack{G \in G \backslash \operatorname{Fix}(f)\\
}} \frac{\operatorname{det}\left(1-T_{x}(f): T_{x}(M) \rightarrow T_{x}(M)\right)}{\left|\operatorname{det}\left(1-T_{x}(f): T_{x}(M) \rightarrow T_{x}(M)\right)\right|} \cdot \overline{\alpha_{x}} \\
& \in \Lambda_{G}(X, f),
\end{aligned}
$$

where for an isomorphism $(\sigma,[t]) \in \operatorname{Mor}(z, x)$ and a path $w_{z}=\left(\operatorname{id},\left[v_{z}\right]\right) \in$ $\operatorname{Mor}(f(z), z)$ the element $\alpha_{x}=\overline{v_{z} f\left(t^{-1}\right) t} \in \mathbb{Z} \pi_{1}\left(X^{G_{x}}(z), z\right)_{\phi^{\prime}}$ is the equivalence class of the following path $\alpha \in \pi_{1}\left(X^{G_{x}}(z), z\right)$ in $X^{G_{x}}(z)$ :

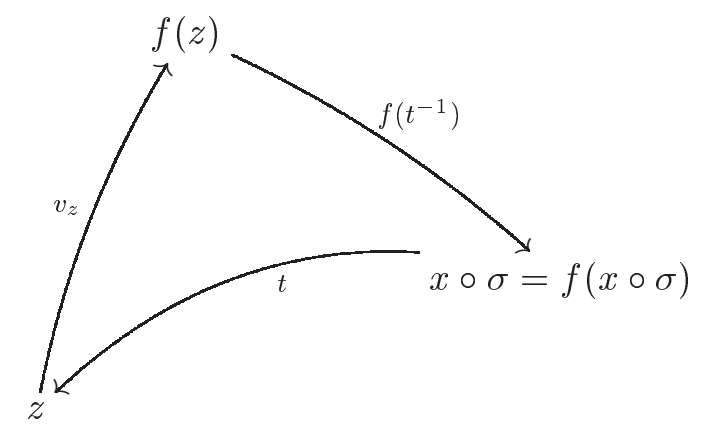

Note that $x \circ \sigma=g_{\sigma} x=g_{\sigma} f(x)=f(x \circ \sigma)$ by $G$-equivariance of $f$, i.e., the whole orbit of a fixed point consists of fixed points.

Remark 8.2.9. We can also prove an equivariant analog of Theorem 1.1.3: If $X$ is a finite proper $G$-CW-complex and $f: X \rightarrow X$ is a $G$-equivariant endomorphism, then $L_{G}(f) \neq 0$ implies that $f$ has an orbit of fixed points. Namely, if $L_{G}(f) \neq 0$, we conclude from injectivity of the character map $\operatorname{ch}^{G}(X): U^{G}(X) \rightarrow \bigoplus_{\text {Is } \Pi(G, X)} \mathbb{Q}[$ LR03, Equation 5.1 and Lemma 5.3] and the fact that [LR03, Lemma 5.4]

$$
\operatorname{ch}^{G}(X)\left(L_{G}(f)\right)_{\bar{y}}= \begin{cases}L^{\mathbb{Q} W K_{y}}\left(\left.f\right|_{X^{K}(y)}\right) & \text { if } X^{K}(f(y))=X^{K}(y) \\ 0 & \text { otherwise }\end{cases}
$$

that there is a $\bar{y} \in \operatorname{Is} \Pi(G, X)$ such that $L^{\mathbb{Q} W K_{y}}\left(\left.f\right|_{X^{K}(y)}\right) \neq 0$. If $\left|W K_{y}\right|<$ $\infty$, then $L^{\mathbb{Q} W K_{y}}\left(\left.f\right|_{X^{K}(y)}\right)=\frac{1}{\left|W K_{y}\right|} L\left(\left.f\right|_{X^{K}(y)}\right)$ [LR03, Remark 1.7], where $L\left(\left.f\right|_{X^{K}(y)}\right)$ denotes the Lefschetz number. So by the Lefschetz fixed point Theorem 1.1.3 we conclude that $\left.f\right|_{X^{K}(y)}$ has a fixed point. If $\left|W K_{y}\right|=$ $\infty$, then we can use a $W K_{y}$-equivariant triangulation of $X^{K}(y)$ and apply barycentric subdivision as in the proof of the Lefschetz fixed point theorem to arrive at the same result. We see that $f$ has a fixed point, and by $G$ equivariance of $f$, the whole orbit of this fixed point consists of fixed points. 


\subsection{The Illustrative Example, yet continued}

In Section 3.2, we introduced as an example the group $\mathbb{Z} / 2$, acting on $S^{1}$ by reflection at the $x$-axis, with the equivariant endomorphism $f: S^{1} \rightarrow$ $S^{1}, z \mapsto z^{d}$ for $d \in \mathbb{N}$. What do the results of this chapter mean in this concrete case?

The most interesting information is given at the "points" $y_{\varphi}: \mathbb{Z} / 2 \rightarrow$ $S^{1}, 1 \mapsto e^{i \varphi}, \tau \mapsto e^{-i \varphi}$. Here we obtain $\mathbb{Z} \Pi\left(\mathbb{Z} / 2, S^{1}\right)_{\phi, y_{\varphi}}=\mathbb{Z} \pi_{1}\left(S^{1}\right) / \sim$, where the equivalence relation is given by $\phi_{y_{\varphi}, w_{y_{\varphi}}}(\gamma) \alpha \gamma^{-1} \sim \alpha$, for $\alpha \in$ $\pi_{1}\left(S^{1}\right) \cong \mathbb{Z}$ and $\gamma \in \operatorname{Aut}\left(y_{\varphi}\right)$, a group extension $1 \rightarrow \mathbb{Z} \rightarrow \operatorname{Aut}\left(y_{\varphi}\right) \rightarrow$ $\mathbb{Z} / 2 \rightarrow 1$. The map $\phi_{y_{\varphi}, w_{y_{\varphi}}}$ is given by multiplication with $d$ on the path factor $\mathbb{Z}$, see Section 3.2. So for $\gamma=(1, m)$ and $\beta=(1, n)$ with $m, n \in \mathbb{Z}$ we have

$$
\begin{array}{rlrl}
\phi_{y_{\varphi}, w_{y_{\varphi}}}(1, m)(1, n)(1, m)^{-1} & \sim(1, n) \\
\Leftrightarrow & (1, d m+n-m) & \sim(1, n),
\end{array}
$$

which implies that $(d-1) m \sim 0$ for all $m \in \mathbb{Z}$. This is the relation also appearing in the non-equivariant case [Lüc99, Chapter 3]. For $\gamma=(\tau, m)$ we obtain

$$
\begin{aligned}
\phi_{y_{\varphi}, w_{y_{\varphi}}}(\tau, m)(1, n)(\tau, m)^{-1} & \sim(1, n) \\
\Leftrightarrow \quad \phi_{y_{\varphi}, w_{y_{\varphi}}}(\tau, m)\left(\tau^{-1},-n-m\right) & \sim(1, n) \\
\Leftrightarrow \quad(1, d m-n-m) & \sim(1, n) .
\end{aligned}
$$

There is a new relation implied by this, namely

$$
-n \sim n \text { for all } n \in \mathbb{Z} .
$$

We obtain

$$
\Lambda_{\mathbb{Z} / 2}\left(X^{1}, f\right)_{y_{\varphi}}=\mathbb{Z} \Pi\left(\mathbb{Z} / 2, S^{1}\right)_{\phi, y_{\varphi}}= \begin{cases}\mathbb{Z}\left[\mathbb{Z} / \frac{d-1}{2}\right] & \text { if } d \neq 1 \text { is odd } \\ \mathbb{Z}[\mathbb{N}] & \text { if } d=1 \\ \mathbb{Z}\left[\mathbb{Z} / \frac{d-2}{2}\right] \oplus \mathbb{Z} / 2 & \text { if } d \text { is even. }\end{cases}
$$

Applying the trace map $\operatorname{tr}_{\mathbb{Z} \operatorname{Aut}\left(y_{\varphi}\right)}$ to $u_{\mathbb{Z} / 2}^{R}\left(S^{1}, f\right)_{y_{\varphi}}$, we obtain for odd $d$

$$
\begin{aligned}
\lambda_{\mathbb{Z} / 2}(f) & =\operatorname{tr}_{\mathbb{Z} \operatorname{Aut}\left(y_{\varphi}\right)}\left(u_{\mathbb{Z} / 2}^{\mathbb{Z}}\left(S^{1}, f\right)\right) \\
& =\operatorname{tr}_{\mathbb{Z} \operatorname{Aut}\left(y_{\varphi}\right)}\left(\operatorname{id}_{R} \cdot(\cdot d)+\operatorname{id}_{R} \cdot\left(\cdot d+\frac{d-1}{2}\right)\right)-\operatorname{tr}_{\mathbb{Z} \operatorname{Aut}\left(y_{\varphi}\right)}\left(d^{\prime}\right) \\
& =\left(\overline{1}+\frac{\overline{d+1}}{2}\right)-\left(\overline{1}+\overline{2}+\ldots+\frac{\overline{d+1}}{2}\right) \\
& =-\left(\overline{2}+\ldots+\frac{\overline{d-1}}{2}\right)
\end{aligned}
$$


for even $d \geq 2$ we have

$$
\begin{aligned}
\lambda_{\mathbb{Z} / 2}(f) & =\operatorname{tr}_{\mathbb{Z} \operatorname{Aut}\left(y_{\varphi}\right)}\left(u_{\mathbb{Z} / 2}^{\mathbb{Z}}\left(S^{1}, f\right)\right) \\
& =\operatorname{tr}_{\mathbb{Z} \operatorname{Aut}\left(y_{\varphi}\right)}\left(\operatorname{id}_{R} \cdot(\cdot d)\right)-\operatorname{tr}_{\mathbb{Z} \operatorname{Aut}\left(y_{\varphi}\right)}\left(d^{\prime \prime}\right) \\
& =\overline{1}-\left(\overline{1}+\overline{2}+\ldots+\frac{\overline{d-1}}{2}\right)+\left(\frac{\bar{d}}{2}\right) \\
& =-\left(\overline{2}+\ldots+\overline{\frac{d-1}{2}}\right)+\left(\frac{\bar{d}}{2}\right)
\end{aligned}
$$

and for $d=0$ we see that

$$
\begin{aligned}
\lambda_{\mathbb{Z} / 2}(f) & =\operatorname{tr}_{\mathbb{Z} \operatorname{Aut}\left(y_{\varphi}\right)}\left(u_{\mathbb{Z} / 2}^{\mathbb{Z}}\left(S^{1}, f\right)\right) \\
& =\operatorname{tr}_{\mathbb{Z} \operatorname{Aut}\left(y_{\varphi}\right)}\left(\operatorname{id}_{R} \cdot(\cdot d)\right)-\operatorname{tr}_{\mathbb{Z} \operatorname{Aut}\left(y_{\varphi}\right)}\left(d^{\prime \prime}\right) \\
& =\overline{0}+\left(\frac{\bar{d}}{2}\right) \\
& =0 .
\end{aligned}
$$

Here the maps $d^{\prime}$ and $d^{\prime \prime}$ appear which were defined in Section 3.2. Note that in the non-equivariant case we have $\Lambda\left(S^{1}, f\right)=\mathbb{Z}[\mathbb{Z} /(d-1)]$, and $\lambda(f)=$ $-(1+2+\ldots+d-1)$ for $d \geq 2, \lambda(f)=1$ if $d=0$, and $\lambda(f)=0$ if $d=1$. How do these compare to the equivariant case? In the equivariant case, the classes always appear in pairs, there is the reflection of the $x$-axis acting. So we only need half as many classes. The fixed points at 1 and -1 account for the other differences. In the light of Chapter 9, the non-vanishing classes correspond to essential fixed points in $S^{1} \backslash\{1,-1\}$. The class at $\overline{1}$ can always be moved to 1 , so it is non-essential. In case $d$ is odd, the class at $\frac{\overline{d-1}}{2}$ can be moved to -1 and is thus non-essential. If $d$ is even, the point -1 seems to account for the summand $\mathbb{Z} / 2$ because it is the cokernel of the map induced by the inclusion $-1 \rightarrow S^{1}$. 


\section{Chapter 9}

\section{Equivariant Nielsen Invariants}

Based on the generalized equivariant Lefschetz invariant $\lambda_{G}(f) \in \Lambda_{G}(X, f)$ developed in Chapter 7, we now introduce equivariant Nielsen invariants called $N_{G}(f)$ and $N^{G}(f)$, which are equivariant analogs of the Nielsen number.

We proceed to show that these Nielsen invariants give minimal bounds for the number of orbits of fixed points in the $G$-homotopy class of $f$. One even obtains results concerning the type and "location" (connected component of the relevant fixed point set) of these fixed point orbits.

There are results on equivariant Nielsen numbers when $G$ is a compact Lie group, for example by Wong [Won93]. One difference between our work and Wong [Won93] is that we also treat infinite discrete groups, another is that our approach is more structural. We introduced the generalized equivariant Lefschetz invariant $\lambda_{G}(f) \in \Lambda_{G}(X, f)$, and we read off the equivariant Nielsen invariants from there instead of defining them ad hoc.

Finally, we prove a converse of the equivariant Lefschetz fixed point theorem: If $X$ is a $G$-Jiang space as defined in Definition 9.4.2, then $L_{G}(f)=$ 0 implies that $f$ is $G$-homotopic to a fixed point free map. Here $L_{G}(f)$ is the equivariant Lefschetz class defined in Definition 7.6.1 [LR03, Definition 3.6].

\subsection{The Nielsen Number}

Classically, the Nielsen number is defined geometrically by counting essential fixed point classes [Jia83, Definition I.4.1]. Alternatively one defines it using the generalized Lefschetz invariant.

Definition 9.1.1. Let $X$ be a finite CW-complex with basepoint $x$, let 
$f: X \rightarrow X$ be an endomorphism, and let

$$
\lambda(f)=\sum_{\bar{\alpha} \in \pi_{1}(X, x)_{\phi}} n_{\bar{\alpha}} \cdot \bar{\alpha} \in \mathbb{Z} \pi_{1}(X, x)_{\phi}
$$

be the generalized Lefschetz invariant associated to $f$. Then the Nielsen number of $f$ is defined by

$$
N(f):=\#\left\{\bar{\alpha} \mid n_{\bar{\alpha}} \neq 0\right\} .
$$

So the Nielsen number is the number of classes in $\pi_{1}(X, x)_{\phi}$ with non-zero coefficients. A class $\alpha$ with non-zero coefficient corresponds to an essential fixed point class in the geometric sense.

\subsection{Equivariant Nielsen Invariants}

In this section, we define equivariant Nielsen invariants. Given the group $\mathbb{Z} \pi_{1}\left(X^{H}(x), x\right)_{\phi^{\prime}}$ defined in Definition 7.1 .4 and an element $\sum_{\bar{\alpha}} n_{\bar{\alpha}} \cdot \bar{\alpha} \in$ $\mathbb{Z} \pi_{1}\left(X^{H}(x), x\right)_{\phi^{\prime}}$, we call a class $\bar{\alpha} \in \pi_{1}\left(X^{H}(x), x\right)_{\phi^{\prime}}$ essential if the coefficient $n_{\bar{\alpha}}$ is non-zero.

We would like to derive equivariant Nielsen invariants from the generalized Lefschetz invariant $\lambda_{G}(f)$ by counting classes with non-zero coefficients. We consider the equation obtained in the refined equivariant Lefschetz fixed point theorem, Theorem 8.2.7. Here $G$ is a discrete group, $M$ is a cocompact proper smooth $G$-manifold and $f: M \rightarrow M$ is a smooth $G$-equivariant map such that $\operatorname{Fix}(f) \cap \partial M=\emptyset$ and such that for every $x \in \operatorname{Fix}(f)$ the determinant of the map $\left(\operatorname{id}_{T_{x} M}-T_{x} f\right)$ is different from zero. We have

$$
\lambda_{G}(f)=\lambda_{G}^{l o c}(f)=\sum_{G x \in G \backslash \operatorname{Fix}(f)} \Lambda_{G}(x, f) \circ \operatorname{ind}_{G_{x} \subseteq G}\left(\operatorname{Deg}_{0}^{G_{x}}\left(\left(\operatorname{id}_{T_{x} M}-T_{x} f\right)^{c}\right)\right) .
$$

We know that $\operatorname{Deg}_{0}^{G_{x}}\left(\left(\operatorname{id}_{T_{x} M}-T_{x} f\right)^{c}\right) \in U^{G_{x}}(*)=A\left(G_{x}\right)$ is a unit of the Burnside ring since $\left(\operatorname{Deg}_{0}^{G_{x}}\left(\left(\mathrm{id}_{T_{x} M}-T_{x} f\right)^{c}\right)\right)^{2}=1$ [LR03, Example 4.7]. We also know that in general a unit of the Burnside ring $A\left(G_{x}\right)$ may consist of more than one summand [tD79]. The summand $\left[G_{x} / G_{x}\right]$ is always included with a coefficient +1 or -1 , but there might be summands $\left[G_{x} / L\right]$ for $L<G_{x}$ appearing. So one fixed point might give more than one class with non-zero coefficients. If we want each fixed point to lead to exactly one summand, we have to divide out the rest, and keep only what corresponds to the summand $\pm 1\left[G_{x} / G_{x}\right]$.

Note that if $\left[G_{x} / L\right] \in U^{G_{x}}(*)$, we have

$$
\Lambda_{G}(x, f) \circ \operatorname{ind}_{G_{x} \subseteq G}\left(\left[G_{x} / L\right]\right)=\left(\operatorname{pr}\left[\operatorname{cst}_{x}\right]\right)^{*}\left(\Lambda_{G}(x, f) \circ \operatorname{ind}_{G_{x} \subseteq G}\left(\left[G_{x} / G_{x}\right]\right)\right),
$$

where pr: $G_{x} / L \rightarrow G_{x} / G_{x}$ denotes the projection. This is the clue to what we have in mind now: We divide out the images of non-isomorphisms. This is 
exactly what the restriction functor $S_{x}$ does, see Definition 1.2.10. We define a reduced variant $\nu(f)$ of the generalized equivariant Lefschetz invariant $\lambda(f)$ to be the image under this quotient map.

Definition 9.2.1. We define the equivariant Nielsen class $\nu_{G}(f)$ by setting

$$
\nu_{G}(f)_{x}:=\overline{\lambda_{G}(f)_{x}} \in S_{x} \mathbb{Z} \Pi(G, X)_{\phi}
$$

for $x \in \Pi(G, X)$. Here $\mathbb{Z} \Pi(G, X)_{\phi}$ is the $\mathbb{Z} \Pi(G, X)$-module defined in Definition 7.1.7 that maps $x: G / H \rightarrow X$ with $X^{H}(f(x))=X^{H}(x)$ to $\mathbb{Z} \pi_{1}\left(X^{H}(x), x\right)_{\phi^{\prime}}$. We set $\mathbb{Z} \pi_{1}\left(X^{H}(x), x\right)_{\phi^{\prime \prime}}:=S_{x} \mathbb{Z} \Pi(G, X)_{\phi}$, for every element $\bar{x} \in \operatorname{Is} \Pi(G, X)$ with $X^{H}(f(x))=X^{H}(x)$.

Let $G$ be a discrete group and let $M$ be a cocompact proper smooth $G$-manifold. Let $f: M \rightarrow M$ be a smooth $G$-equivariant map such that $\operatorname{Fix}(f) \cap \partial M=\emptyset$ and such that for every $x \in \operatorname{Fix}(f)$ the determinant of the $\operatorname{map}\left(\operatorname{id}_{T_{x} M}-T_{x} f\right)$ is different from zero. Then from Theorem 8.2.7 and the definition of the equivariant Nielsen class $\nu_{G}(f)$ we derive the equation

$$
\nu_{G}(f)=\sum_{G x \in G \backslash \operatorname{Fix}(f)} \frac{\operatorname{det}\left(1-T_{x}(f): T_{x}(M) \rightarrow T_{x}(M)\right)}{\left|\operatorname{det}\left(1-T_{x}(f): T_{x}(M) \rightarrow T_{x}(M)\right)\right|} \cdot \overline{\alpha_{x}} .
$$

We will now define equivariant Nielsen invariants by counting the essential classes $\bar{\alpha}$ of $\nu_{G}(f)_{\bar{x}}$ in $\mathbb{Z} \pi_{1}\left(X^{H}(x), x\right)_{\phi^{\prime \prime}}$ and of $\operatorname{ch}_{G}(X, f)\left(\lambda_{G}(f)\right)_{\bar{x}}$ in $\mathbb{Q} \pi_{1}\left(X^{H}(x), x\right)_{\phi^{\prime}}$, for $\bar{x} \in \operatorname{Is} \Pi(G, X)$.

Remember from the definition of the fundamental category $\Pi(G, X)$, Definition 1.2.2, that its objects are $G$-equivariant maps $x: G / H \rightarrow X$ for subgroups $H \leq G$, and that we often view $x$ as the point $x(1 H) \in X^{H}$. We call $X^{H}(x)$ the connected component of $X^{H}$ containing $x(1 H)$. In order to simplify notation, we will use $f^{H}(x)$ to denote $\left.f\right|_{X^{H}(x)}$ from now on, and we will use $f_{H}(x)$ instead of $\left.\left.f\right|_{\left(X^{H}(x), X>H\right.}(x)\right)$.

Definition 9.2.2. Let $G$ be a discrete group, let $X$ be a finite proper $G$ CW-complex, and let $f: X \rightarrow X$ be a $G$-equivariant map.

We define equivariant Nielsen invariants of $f$ to be the elements

$$
N_{G}(f), N^{G}(f) \in \bigoplus_{\bar{x} \in \Pi(G, X)} \mathbb{Z}
$$

defined by

$$
\begin{aligned}
N_{G}(f)_{\bar{x}}:=\#\left\{\text { essential classes of } \nu_{G}(f)_{\bar{x}}\right\} \\
N^{G}(f)_{\bar{x}}:=\min \left\{\# \mathcal{C} \mid \mathcal{C} \subseteq \bigcup_{y \geq x} \pi_{1}\left(X^{K}(y), y\right)_{\phi^{\prime}} \text { such that for all } \bar{z} \geq \bar{x}\right. \\
\text { we have }\left\{\text { essential classes } \bar{\alpha} \text { of } \operatorname{ch}_{G}(X, f)\left(\lambda_{G}(f)\right)_{\bar{z}}\right\} \\
\left.\subseteq\left\{(\sigma,[t])^{*}(\bar{\beta}) \mid \bar{\beta} \in \mathcal{C},(\sigma,[t]) \in \operatorname{Mor}\left(z, y_{\bar{\beta}}\right)\right\}\right\}
\end{aligned}
$$


for $\bar{x} \in \operatorname{Is} \Pi(G, X)$ with $X^{H}(f(x))=X^{H}(x)$, and by 0 for $\bar{x} \in \operatorname{Is} \Pi(G, X)$ with $X^{H}(f(x)) \neq X^{H}(x)$.

Note that $N_{G}(f)_{\bar{x}}=N_{G}\left(f_{H}(x)\right)$ and $N^{G}(f)_{\bar{x}}=N O_{G}\left(f^{H}(x)\right)$ in the notation of Wong [Won93].

An essential class $\bar{\alpha}$ of $\operatorname{ch}_{G}(X, f)\left(\lambda_{G}(f)\right)_{\bar{x}}=L^{\mathbb{Q} A u t(x)}\left(\widetilde{f^{H}(x)}\right)$ corresponds to an essential fixed point class of $f^{H}(x)$, a $W H_{x}$-orbit of fixed points which one cannot get rid of under any $G$-homotopy, compare the refined orbifold Lefschetz fixed point theorem, Theorem 8.1.1. An essential class $\bar{\alpha}$ of $\nu_{G}(f)_{\bar{x}}$ corresponds to an essential fixed point class of $f_{H}(x)$, an orbit of fixed points on $X^{H}(x) \backslash X^{>H}(x)$ that cannot be moved into $X^{>H}(x)$. Counting the essential classes will give us information on the number of fixed points and fixed point orbits.

The equivariant Nielsen invariants are $G$-homotopy invariant since they are derived from $\lambda_{G}(f)$, which is itself $G$-homotopy invariant:

Proposition 9.2.3. Given a $G$-homotopy $f \simeq_{G} f^{\prime}$, we have

$$
\begin{aligned}
& N_{G}(f)=N_{G}\left(f^{\prime}\right) \\
& N^{G}(f)=N^{G}\left(f^{\prime}\right) .
\end{aligned}
$$

Proof. If $f \simeq_{G} f^{\prime}$, with a homotopy $H: X \times I \rightarrow X$ such that $H_{0}=f$ and $H_{1}=f^{\prime}$, then by invariance under homotopy equivalence we have an isomorphism $\Lambda_{G}\left(i_{1}\right)^{-1} \Lambda_{G}\left(i_{0}\right): \Lambda_{G}(X, f) \stackrel{\sim}{\rightarrow} \Lambda_{G}\left(X, f^{\prime}\right)$ which sends $\lambda_{G}(f)$ to $\lambda_{G}\left(f^{\prime}\right)$. The isomorphisms $\Lambda_{G}\left(i_{1}\right)$ and $\Lambda_{G}\left(i_{0}\right)$ are given by composition of maps, so they do not change the number of essential classes. They also do not change the property of a class to lie in the image of a non-isomorphism. So we have $N_{G}(f)=N_{G}\left(f^{\prime}\right)$.

An isomorphism $i_{0 *}: \mathbb{Q} \Pi(G, X)_{\phi, \bar{y}} \rightarrow \mathbb{Q} \Pi(G, X \times I)_{\Phi, \overline{i_{0}(y)}}$ is induced by the inclusion $i_{0}$, and analogously $i_{1}$ induces an isomorphism. These isomorphisms do not change the number of essential classes. We have $\operatorname{ch}_{G}(X, f)\left(\lambda_{G}(f)\right)=\left(i_{0 *}\right)^{-1} i_{1 *} \operatorname{ch}_{G}\left(X, f^{\prime}\right)\left(\lambda_{G}\left(f^{\prime}\right)\right)$, so $N^{G}(f)=N^{G}\left(f^{\prime}\right)$.

\subsection{Lower Bound Property}

The equivariant Nielsen invariants give a lower bound for the number of fixed point orbits on the fixed point sets $X_{H}(x)$ and $X^{H}(x)$, for maps lying in the $G$-homotopy class of $f$, and under mild hypotheses this is even a sharp lower bound.

Definition 9.3.1. Let $G$ be a discrete group, let $X$ be a finite proper $G$ CW-complex, and let $f: X \rightarrow X$ be a $G$-equivariant map. For every $\bar{x} \in$ Is $\Pi(G, X)$, with $x: G / H \rightarrow X$, we set

$$
\begin{aligned}
& M_{G}(f)_{\bar{x}}:=\min \left\{\# \text { fixed point orbits of } \varphi_{H}(x) \mid \varphi \simeq_{G} f\right\} \\
& M^{G}(f)_{\bar{x}}:=\min \left\{\# \text { fixed point orbits of } \varphi^{H}(x) \mid \varphi \simeq_{G} f\right\} .
\end{aligned}
$$


When speaking of fixed point orbits of $f^{H}(x)$, we can either look at the $W H_{x}$-orbits $W H_{x} \cdot z \subseteq X^{H}(x)$ or at the $G$-orbits $G \cdot z \subseteq X^{(H)}(x)$, for a fixed point $z$ in $X^{H}(x)$. These two notions are of course equivalent.

We now proceed to show the first important property of the equivariant Nielsen invariants, the lower bound property.

Proposition 9.3.2. For every $\bar{x} \in \operatorname{Is} \Pi(G, X)$ we have

$$
\begin{aligned}
N_{G}(f)_{\bar{x}} & \leq M_{G}(f)_{\bar{x}} \\
N^{G}(f)_{\bar{x}} & \leq M^{G}(f)_{\bar{x}} .
\end{aligned}
$$

Proof. 1) If $\bar{\alpha} \in \pi_{1}\left(X^{H}(x), x\right)_{\phi^{\prime \prime}}$ is an essential class of $\nu_{G}(f)_{\bar{x}}$, then there has to be at least one fixed point orbit in $X^{H}(x) \backslash X^{>H}(x)$ that corresponds to $\bar{\alpha}$ and that cannot be moved into $X^{>H}(x)$. So, for any $\varphi \simeq_{G} f$, the restriction $\varphi_{H}$ must have at least $N_{G}(f)_{\bar{x}}$ fixed point orbits in $X^{H}(x) \backslash$ $X^{>H}(x)$. We arrive at $N_{G}(f)_{\bar{x}} \leq\left\{\#\right.$ fixed point orbits of $\left.\varphi_{H}\right\}$ for all $\varphi \simeq_{G}$ $f$, so $N_{G}(f)_{\bar{x}} \leq M_{G}(f)_{\bar{x}}$.

2) Let $\bar{x} \in \operatorname{Is} \Pi(G, X)$. Suppose that $\varphi \simeq_{G} f$ such that $\varphi^{H}(x)$ has $M O^{G}(f)_{\bar{x}}$ fixed point orbits in $X^{H}(x)$. Let $\mathcal{C} \subseteq \bigcup_{\bar{x}<\bar{y}} \pi_{1}\left(X^{K}(y), y\right)_{\phi^{\prime}}$ such that $N O^{G}(\varphi)_{\bar{x}}=N O^{G}(f)_{\bar{x}}=\# \mathcal{C}$. If there were less than $\# \mathcal{C}$ fixed point orbits in $X^{H}(x)$, there would be less that $\# \mathcal{C}$ essential classes and we could have chosen a smaller $\mathcal{C}$. So there are at least $\# \mathcal{C}$ essential classes, and thus $\varphi^{H}(x)$ has at least $\# \mathcal{C}$ fixed point orbits.

To prove the sharpness of this lower bound, we need certain hypotheses, which are usually introduced when dealing with these problems. Other authors treat slightly weakened assumptions [Fer99, Fer03, Jez95, Wil84]. We do not weaken the standard gap hypotheses in the context of functorial equivariant Lefschetz invariants since the standard gap hypotheses are not homotopy invariant. So an analog of Theorem 9.5.3 would not hold.

Definition 9.3.3. Let $G$ be a discrete group and let $X$ be a cocompact smooth $G$-manifold. We say that $X$ satisfies the standard gap hypotheses if for each $\bar{x} \in \operatorname{Is} \Pi(G, X)$, with $x: G / H \rightarrow X$, the inequalities $\operatorname{dim} X^{H}(x) \geq 3$ and $\operatorname{dim} X^{H}(x)-\operatorname{dim} X^{>H}(x) \geq 2$ hold.

Under these hypotheses, we can use an equivariant analog of the classical Wecken method [Wec41] to coalesce fixed points.

Lemma 9.3.4. Let $G$ be a discrete group and let $X$ be a cocompact proper smooth $G$-manifold satisfying the standard gap hypotheses. Let $f: X \rightarrow X$ be a $G$-equivariant map. Let $\mathcal{O}_{1}=G x_{1}$ and $\mathcal{O}_{2}=G x_{2}$ be two distinct isolated $G$-fixed point orbits, where $x_{1}: G / H \rightarrow X$ and $x_{2}: G / K \rightarrow X$ with $x_{1} \leq x_{2}$. Suppose that there are paths $\left(\sigma_{1},\left[t_{1}\right]\right) \in \operatorname{Mor}\left(x, x_{1}\right)$ and $\left(\sigma_{2},\left[t_{2}\right]\right) \in \operatorname{Mor}\left(x, x_{2}\right)$ for an $\bar{x} \in \Pi(G, X)$, with $x: G / H \rightarrow X$, such that $\left(\sigma_{1},\left[t_{1}\right]\right)^{*} \overline{1_{x_{1}}}=\bar{\alpha}=\left(\sigma_{2},\left[t_{2}\right]\right)^{*} \overline{1_{x_{2}}}$, i.e., that the fixed point orbits induce the 
same $\bar{\alpha} \in \pi_{1}\left(X^{H}(x), x\right)_{\phi^{\prime}}$. Then there exists a $G$-homotopy $\left\{f_{t}\right\}$ relative to $X^{>(H)}$ such that $f_{0}=f$ and $\operatorname{Fix} f_{1}=\operatorname{Fix} f_{0}-G \mathcal{O}_{1}$.

Proof. Suppose first that $\overline{x_{1}}<\overline{x_{2}}$. Then $\operatorname{Mor}\left(x_{1}, x_{2}\right) \neq \emptyset$. By replacing $x_{1}$ and $x_{2}$ with other points in the orbit if necessary, we can suppose that there exists a morphism $(\tau,[v]) \in \operatorname{Mor}\left(x_{1}, x_{2}\right)$, where $v$ is a path in $X^{H}(x)$ with $v_{1}=x_{1}$ and $v_{0}=x_{2} \circ \tau$ and $\tau: G / H \rightarrow G / K$ is a projection. We know that $v \simeq f^{H} \circ v$ (relative endpoints). (This is an equivalent characterization of $x_{1}$ and $x_{2}$ belonging to the same fixed point class [Jia83, I.1.10].) Since $x_{1} \in X^{H}(x) \backslash X^{>H}(x)$ and $x_{2} \in X^{>H}(x)$ and $\operatorname{dim} X^{H}(x)-\operatorname{dim} X^{>H}(x) \geq 2$, we may assume that $v$ can be chosen such that $v((0,1]) \subseteq X^{H}(x) \backslash X^{>H}(x)$. We coalesce $x_{1}$ and $x_{2}$ along $v$ as in [Won91b, 1.1], [Sch86, 6.1]. We can do this by only changing $f$ in a (cone-shaped) neighborhood $U(v)$ of $v$. Because of the proper action of $G$ on $X$ and the free action of $W H$ on $X^{H} \backslash X^{>H}$, this neighborhood $U(v)$ can be chosen such that in $X^{H} \backslash X^{>H}$ it does not intersect its $g$-translates for $g \notin H \leq G$. Taking the $G$-translates of $U(v)$, we move $\mathcal{O}_{1}$ to $\mathcal{O}_{2}$ along the paths $G v$ in $G U(v)$, not changing the map $f$ outside $G U(v)$.

Now suppose $\overline{x_{1}}=\overline{x_{2}}$. In this case, the result follows from the result of Wong [Won91a, 5.4] since $X^{H}(x) \backslash X^{>H}(x)$ is a free and proper $W H_{x^{-}}$ space, where again the proper action of $G$ on $X$ ensures that we can find a neighborhood of a path from $x_{1}$ to $x_{2}$ such that the $G / H$-translates do not intersect.

From Lemma 9.3.4, we can conclude the sharpness of the lower bound given by the equivariant Nielsen invariants.

Theorem 9.3.5. Let $G$ be a discrete group. Let $X$ be a cocompact proper smooth $G$-manifold satisfying the standard gap hypotheses. Let $f: X \rightarrow X$ be a G-equivariant endomorphism. Then

$$
\begin{aligned}
M_{G}(f)_{\bar{x}} & =N_{G}(f)_{\bar{x}} \\
M^{G}(f)_{\bar{x}} & =N^{G}(f)_{\bar{x}}
\end{aligned}
$$

for all $\bar{x} \in \operatorname{Is} \Pi(G, X)$.

Proof. 1) Since $X$ is a cocompact smooth $G$-manifold, there is a $G$-map $f^{\prime}$ which is $G$-homotopic to $f$ and which has only finitely many fixed point orbits. We apply Lemma 9.3.4 to $f^{\prime}$ to coalesce fixed point orbits in $X^{H}(x) \backslash$ $X^{>H}(x)$ with others of the same class $\bar{\alpha} \in \mathbb{Z} \pi_{1}\left(X^{H}(x), x\right)_{\phi^{\prime}}$. We move them into $X^{>H}(x)$ whenever possible. (We might need to create a fixed point orbit in the inessential fixed point class before [Won91b, 1.1].) We remove the inessential fixed point orbits. We arrive at a map $h \simeq_{G} f$ such that $N_{G}(f)_{\bar{x}}=\#\left\{\right.$ fixed point orbits of $\left.h_{H}(x)\right\} \geq M_{G}(f)_{\bar{x}}$. Using Proposition 9.3.2, we obtain equality. 
2) Since $X$ is a cocompact smooth $G$-manifold, there is a map $f^{\prime}$ which is $G$-homotopic to $f$ and which only has finitely many fixed point orbits. We have a partial ordering on the $\bar{y} \geq \bar{x}$ given by $\bar{y} \geq \bar{z} \Leftrightarrow \operatorname{Mor}(z, y) \neq \emptyset$. We apply Lemma 9.3.4 to $f^{\prime}$ to coalesce fixed point orbits of the same class, starting from the top. Note that when we remove fixed point orbits, we can only move them up in this partial ordering. That is why the definition has to be so complicated. We remove the inessential fixed point orbits. We are left with one fixed point orbit for every essential class.

We now look at a class $\mathcal{C}$ such that $N O^{G}(f)_{\bar{x}}=\# \mathcal{C}$, and we coalesce the essential fixed point orbits with the corresponding classes appearing in $\mathcal{C}$. (If the corresponding class in $\mathcal{C}$ is inessential, we might need to create a fixed point orbit in this inessential fixed point class before [Won91b, 1.1].) We obtain a map $h \simeq_{G} f$ which has exactly $\# \mathcal{C}$ fixed point orbits. Hence $N O^{G}(f)_{\bar{x}}=\#\left\{\right.$ fixed point orbits of $\left.h^{H}(x)\right\} \geq M O^{G}(f)_{\bar{x}}$. Using proposition 9.3.2, we obtain equality.

In general, it is not possible to find a map $h \simeq_{G} f$ realizing all minima simultaneously. As an example, one can take $G=\mathbb{Z} / 2$ acting on $X=S^{4}$ as an involution so that $X^{\mathbb{Z} / 2}=S^{3}$. One obtains $M_{G}\left(\operatorname{id}_{S^{4}}\right)_{\bar{x}}=0$ for all $\bar{x} \in$ Is $\Pi\left(\mathbb{Z} / 2, S^{4}\right)$, but the minimal number of fixed points in the $G$-homotopy class of the identity $\operatorname{id}_{S^{4}}$ is equal to 1 [Won93, Remark 3.4]. In this example, the standard gap hypotheses are not satisfied.

\subsection{The G-Jiang Condition}

In the non-equivariant case, we know that the generalized Lefschetz invariant is the right element when looking for a precise count of fixed points. We read off the Nielsen numbers from this invariant. In general, the Lefschetz number contains too little information. But under certain conditions, we can conclude facts about the Nielsen numbers from the Lefschetz numbers directly, and thus obtain a converse of the Lefschetz fixed point theorem.

These conditions are called Jiang conditions. See Jiang [Jia83, Definition II.4.1], where one can find a thorough treatment, and Brown [Bro71, Chapter VII]. The Jiang group is a subgroup of $\pi_{1}(X, f(x))$ [Jia83, Definition II.3.5]. We generalize its definition to the equivariant case.

Definition 9.4.1. Let $G$ be a discrete group, let $X$ be a finite proper $G$ CW-complex, and let $f: X \rightarrow X$ be a $G$-equivariant endomorphism. Then a $G$-equivariant self-homotopy $h: f \simeq_{G} f$ of $f$ determines a path $h(x,-) \in$ $\pi_{1}\left(X^{H}(x), f(x)\right)$ for every $\bar{x} \in \operatorname{Is} \Pi(G, X)$, with $x: G / H \rightarrow X$. Define the 
$G$-Jiang group of $(X, f)$ to be

$$
\begin{aligned}
J_{G}(X, f) & :=\left\{\sum_{\bar{x} \in \Pi(G, X)}[h(x,-)] \mid h: f \simeq_{G} f G \text {-equivariant self-homotopy }\right\} \\
& \leq \bigoplus_{\bar{x} \in \Pi(G, X)} \pi_{1}\left(X^{H}(x), f(x)\right)
\end{aligned}
$$

and define the $G$-Jiang group of $X$ to be

$$
\begin{aligned}
J_{G}(X) & :=\left\{\sum_{\bar{x} \in \Pi(G, X)}[h(x,-)] \mid h: \text { id } \simeq_{G} \text { id } G \text {-equivariant self-homotopy }\right\} \\
& \leq \bigoplus_{\bar{x} \in \Pi(G, X)} \pi_{1}\left(X^{H}(x), x\right)
\end{aligned}
$$

In the non-equivariant case, we know that the Jiang group $J(X, f, x)$ is a subgroup of the centralizer of $\pi_{1}(f, x)\left(\pi_{1}(X, x)\right)$ in $\pi_{1}(X, f(x))$. In particular, $J(X) \leq Z\left(\pi_{1}(X, x)\right)$, where $Z\left(\pi_{1}(X, x)\right)$ denotes the center of $\pi_{1}(X, x)$ [Jia83, Lemma II.3.7]. Furthermore, the isomorphism ( $f \circ$ $w)_{*}: \pi_{1}\left(X, f\left(x_{1}\right)\right) \rightarrow \pi_{1}\left(X, f\left(x_{0}\right)\right)$ induced by a path $w$ from $x_{0}$ to $x_{1}$ induces an isomorphism $(f \circ w)_{*}: J\left(X, f, x_{1}\right) \rightarrow J\left(X, f, x_{0}\right)$ which does not depend on the choice of $w$. So the definition does not depend on the choice of the basepoint [Jia83, Lemma II.3.9]. It is also known that $J(X) \leq J(X, f) \leq \pi_{1}(X)$ for all $f$ [Jia83, Lemma II.3.8]. This leads to the consideration of spaces with $J(X)=\pi_{1}(X)$ in the definition of a Jiang space. All these lemmata also make sense in the equivariant case. Thus we make the following definition.

Definition 9.4.2. Let $G$ be a discrete group and let $X$ be a cocompact $G$-CW-complex. Then $X$ is called a $G$-Jiang space if for all $\bar{x} \in \operatorname{Is} \Pi(G, X)$ we have

$$
J_{G}(X)_{\bar{x}}=\pi_{1}\left(X^{H}(x), x\right) .
$$

The group $J_{G}(X, f)$ acts on $\Lambda_{G}(X, f)$ as follows: If $X^{H}(f(x))=X^{H}(x)$ and $X^{H}(f(x))=X^{H}(x)$, then $J_{G}(X, f)_{\bar{x}}$ acts on $\mathbb{Z} \pi_{1}\left(X^{H}(x), x\right)$. The element $v=[h(x,-)] \in J_{G}(X, f)_{\bar{x}}$ acts as composition with $\left[v_{x} v v_{x}^{-1}\right]$, where $w_{x}=\left(\mathrm{id},\left[v_{x}\right]\right) \in \operatorname{Mor}(f(x), x)$. Since $J_{G}(X, f)_{\bar{x}}$ is contained in the centralizer of $\pi_{1}\left(f^{H}(x), x\right)\left(\pi_{1}\left(X^{H}(x), x\right)\right)$ in $\pi_{1}\left(X^{H}(x), f(x)\right)$, this action induces an action on $\mathbb{Z} \pi_{1}\left(X^{H}(x), x\right)_{\phi_{x, w_{x}}^{\prime}}$ by composition, whence on $\Lambda_{G}(X, f)_{\bar{x}}$. Thus $J_{G}(X, f)$ acts on $\Lambda_{G}(X, f)$, and by invariance of $\lambda_{G}(f)$ under homotopy equivalence, we see that

$$
\lambda_{G}(f) \in\left(\Lambda_{G}(X, f)\right)^{J_{G}(X, f)} .
$$

Examples for $G$-Jiang spaces can be obtained from Jiang spaces. It is known [Jia83, Theorem II.3.11] that the class of Jiang spaces is closed under homotopy equivalence and the topological product operation and contains 
- simply connected spaces

- generalized lens spaces

- H-spaces

- homogeneous spaces of the form $A / A_{0}$ where $A$ is a topological group and $A_{0}$ is a subgroup which is a connected compact Lie group.

Hence we obtain many examples of $G$-Jiang spaces using the following proposition, analogous to Wong [Won93, Proposition 4.9].

Proposition 9.4.3. Let $G$ be a discrete group, and let $X$ be a free cocompact connected proper $G$-space. If $X / G$ is a Jiang space, then $X$ is a G-Jiang space.

Proof. Since $X$ is connected and free, the set $\operatorname{Is}(G, X)$ consists of one element. Let $x$ be a basepoint of $X$. We need to check that $J_{G}(X)_{\bar{x}}=\pi_{1}(X, x)$. Let $X \stackrel{p}{\rightarrow} X / G$ be the projection. The Jiang subgroup of $X / G$ is given by

$$
\begin{aligned}
J(X / G) & :=\left\{[h(p(x),-)] \mid h: \mathrm{id}_{X / G} \simeq \mathrm{id}_{X / G} \text { self-homotopy }\right\} \\
& \leq \pi_{1}(X / G, p(x)) .
\end{aligned}
$$

Let $\alpha \in \pi_{1}(X, x)$. Since $X \stackrel{p}{\rightarrow} X / G$ is a discrete cover, $\widetilde{X}=\widetilde{X / G}$. There is a surjective map $p_{\#}: \pi_{1}(X, x) \rightarrow \pi_{1}(X / G, p(x))$. Since $X / G$ is a Jiang space, $J(X / G)=\pi_{1}(X / G, p(x))$, so there is a homotopy $h: \mathrm{id}_{X / G} \simeq \operatorname{id}_{X / G}$ such that $p_{\#}(\alpha)=[h(p(x),-)]$. Because of the free and proper action of $G$ on $X$, this homotopy $h$ can be lifted to a $G$-equivariant homotopy $h^{\prime}: \operatorname{id}_{X} \simeq_{G} \operatorname{id}_{X}$ such that $\alpha=\left[h^{\prime}(x,-)\right]$. Thereby $\alpha \in J_{G}(X)$.

\subsection{The Converse of the Equivariant Lefschetz Fixed Point Theorem}

One can derive equivariant analogs of statements about Nielsen numbers found in Jiang [Jia83], generalizing results of Wong [Won93] to infinite discrete groups. In particular, if $X$ is a $G$-Jiang space, the converse of the equivariant Lefschetz fixed point theorem holds. We have the following theorem, compare Jiang [Jia83, Theorem II.4.1].

Theorem 9.5.1. Let $G$ be a discrete group, and let $X$ be a finite proper $G$-CW-complex which is a $G$-Jiang space. Then for any $G$-map $f: X \rightarrow X$ and $\bar{x} \in \operatorname{Is} \Pi(G, X)$ with $x: G / H \rightarrow X$ we have:

$$
\begin{aligned}
& L_{G}(f)_{\bar{x}}=0 \quad \Longrightarrow \quad \lambda_{G}(f)_{\bar{x}}=0 \text { and } N_{G}(f)_{\bar{x}}=0, \\
& L_{G}(f)_{\bar{x}} \neq 0 \quad \Longrightarrow \quad \lambda_{G}(f)_{\bar{x}} \neq 0 \text { and } N_{G}(f)_{\bar{x}}=\#\left\{\pi_{1}\left(X^{H}(x), x\right)_{\phi^{\prime \prime}}\right\} .
\end{aligned}
$$

Here $L_{G}(f)$ is the equivariant Lefschetz class defined in Definition 7.6 .1 [LR03, Definition 3.6], the equivariant analog of the Lefschetz number. 
Proof. Since $X$ is a $G$-Jiang space, the $G$-Jiang group $J_{G}(X)$ acts transitively on $\pi_{1}\left(X^{H}(x), x\right)$ for all $\bar{x} \in \operatorname{Is}(G, X)$. This implies that

$$
\lambda_{G}(f)_{\bar{x}}=\sum_{\bar{\alpha}} n_{\bar{\alpha}} \cdot \bar{\alpha}=n \cdot \sum_{\bar{\alpha}} \bar{\alpha}
$$

for some $n \in \mathbb{Z}$. This leads to $L_{G}(f)_{\bar{x}}=n \cdot \#\left\{\pi_{1}\left(X^{H}(x), x\right)_{\phi^{\prime}}\right\}$ by the augmentation map introduced in Section 7.6. We see that

$$
\begin{aligned}
L_{G}(f)_{\bar{x}}=0 & \Longrightarrow n=0 \\
& \Longrightarrow \lambda_{G}(f)_{\bar{x}}=0 \\
& \Longrightarrow \nu_{G}(f)_{\bar{x}}=0 \\
& \Longrightarrow N_{G}(f)_{\bar{x}}=0 \\
L_{G}(f)_{\bar{x}} \neq 0 & \Longrightarrow n \neq 0 \\
& \Longrightarrow \lambda_{G}(f)_{\bar{x}, \bar{\alpha}} \neq 0 \text { for all } \bar{\alpha} \in \mathbb{Z}\left(\pi_{1}\left(X^{H}(x), x\right)\right)_{\phi^{\prime}} \\
& \Longrightarrow \nu_{G}(f)_{\bar{x}, \bar{\alpha}} \neq 0 \text { for all } \bar{\alpha} \in \mathbb{Z}\left(\pi_{1}\left(X^{H}(x), x\right)\right)_{\phi^{\prime \prime}} \\
& \Longrightarrow N_{G}(f)_{\bar{x}}=\#\left\{\pi_{1}\left(X^{H}(x), x\right)_{\phi^{\prime \prime}}\right\} .
\end{aligned}
$$

The proof of Theorem 9.5.1 already works if $J_{G}(X, f)$ acts transitively on every summand of $\Lambda_{G}(X, f)$. We could have called $X$ a $G$-Jiang space if the condition that $J_{G}(X, f)$ acts transitively on every summand of $\Lambda_{G}(X, f)$ is satisfied. But this condition is less tractable. It is implied by $J_{G}(X, f)_{\bar{x}}=$ $\pi_{1}\left(X^{H}(x), f(x)\right)$ for all $\bar{x}$, which is implied by $J_{G}(X)_{\bar{x}}=\pi_{1}\left(X^{H}(x), x\right)$ for all $\bar{x}$.

We now show that $f$ is $G$-homotopic to a fixed point free $G$-map if the generalized equivariant Lefschetz invariant $\lambda_{G}(f)$ is zero.

Theorem 9.5.2. Let $G$ be a discrete group. Let $X$ be a cocompact proper smooth $G$-manifold satisfying the standard gap hypotheses. Let $f: X \rightarrow X$ be a $G$-equivariant endomorphism. Then the following holds:

If $\lambda_{G}(f)=0$, then $f$ is $G$-homotopic to a fixed point free $G$-map.

Proof. If $\lambda_{G}(f)=0$, then $\operatorname{ch}_{G}(X, f)\left(\lambda_{G}(f)\right)=0$, and therefore we have $N^{G}(f)_{\bar{x}}=0$ for all $\bar{x} \in \operatorname{Is}(G, X)$. We know from Theorem 9.3.5 that $N^{G}(f)_{\bar{x}}=M^{G}(f)_{\bar{x}}=\min \left\{\#\right.$ fixed point orbits of $\left.\varphi^{H}(x) \mid \varphi \simeq_{G} f\right\} . \quad$ In particular, for $x: G /\{1\} \rightarrow X$ we obtain a map $\varphi$ such that $\varphi^{\{1\}}(x)$ is fixed point free and $\varphi \simeq_{G} f$. Thus we obtain our result on every connected component of $X$, and combining these we arrive at a map $h \simeq_{G} f$ which is fixed point free.

These two theorems, Theorem 9.5.1 and Theorem 9.5.2, combine to give the main theorem of this chapter, the converse of the equivariant Lefschetz fixed point theorem. 
Theorem 9.5.3. Let $G$ be a discrete group. Let $X$ be a cocompact proper smooth G-manifold satisfying the standard gap hypotheses which is a $G$ Jiang space. Let $f: X \rightarrow X$ be a G-equivariant endomorphism. Then the following holds:

If $L_{G}(f)=0$, then $f$ is $G$-homotopic to a fixed point free $G$-map.

Proof. We know that $L_{G}(f)=0$ means that $L_{G}(f)_{\bar{x}}=0$ for all $\bar{x} \in \operatorname{Is}(G, X)$. Since $X$ is a $G$-Jiang space, by Theorem 9.5.1 this implies $\lambda_{G}(f)_{\bar{x}}=0$ for all $\bar{x} \in \operatorname{Is}(G, X)$, so we have $\lambda_{G}(f)=0$. We apply Theorem 9.5.2 to arrive at the desired result.

Remark 9.5.4. As another corollary of Theorem 9.5.2, we obtain: If $G$ is a discrete group and $X$ is a cocompact proper smooth $G$-manifold satisfying the standard gap hypotheses, then $\chi^{G}(X)=0$ implies that the identity id $_{X}$ is $G$-homotopic to a fixed point free $G$-map. This was already stated by Lück and Rosenberg [LR03, Remark 6.8]. Here $\chi^{G}(X) \in U^{G}(X)$ is the universal equivariant Euler characteristic of $X$ [LR03, Definition 6.1] defined by $\chi^{G}(X)_{\bar{x}}=\chi\left(W H_{x} \backslash X^{H}(x), W H_{x} \backslash X^{>H}(x)\right) \in \mathbb{Z}$, we have $\chi^{G}(X)=$ $L_{G}\left(\operatorname{id}_{X}\right)$. We calculate that

$$
\begin{aligned}
\lambda_{G}\left(\operatorname{id}_{X}\right)_{\bar{x}} & =\sum_{p \geq 0}(-1)^{p} \sum_{\substack{\operatorname{Aut}(x) \cdot e \epsilon \\
\operatorname{Aut}(x) \backslash I_{p}\left(X^{H}(x), X \mathcal{H}^{\prime}(x)\right)}} \operatorname{inc}_{\phi}\left(\operatorname{id}_{\widetilde{X^{H}(x)}}, e\right) \\
& =\chi\left(W H_{x} \backslash X^{H}(x), W H_{x} \backslash X^{>H}(x)\right) \cdot \overline{1} \in \mathbb{Z} \pi_{1}\left(X^{H}(x), x\right)_{\phi^{\prime}} .
\end{aligned}
$$

So we have $\chi^{G}(X)=0$ if and only if $\lambda_{G}\left(\operatorname{id}_{X}\right)=0$, and with Theorem 9.5.2 we conclude that there is an endomorphism $G$-homotopic to the identity which is fixed point free. 


\section{Bibliography}

[Alm74] Gert Almkvist. The Grothendieck ring of the category of endomorphisms. J. Algebra, 28:375-388, 1974.

[Bas68] Hyman Bass. Algebraic K-Theory. W. A. Benjamin, Inc., New York-Amsterdam, 1968.

[Bre72] Glen E. Bredon. Introduction to Compact Transformation Groups. Academic Press, New York, 1972. Pure and Applied Mathematics, Vol. 46.

[Bro71] Robert F. Brown. The Lefschetz Fixed Point Theorem. Scott, Foresman and Co., Glenview, Ill.-London, 1971.

[Bru04] Morten Brun. Witt vectors and $K$-theory of endomorphisms. Private communication, 2004.

[Bru05] Morten Brun. On the Lück-Weber splitting of $K$-theory. Private communication, 2005.

[CP97] M. Cárdenas and E. K. Pedersen. On the Karoubi filtration of a category. K-Theory, 12(2):165-191, 1997.

[DL98] James F. Davis and Wolfgang Lück. Spaces over a category and assembly maps in isomorphism conjectures in $K$ - and $L$-theory. K-Theory, 15(3):201-252, 1998.

[DWW03] W. Dwyer, M. Weiss, and B. Williams. A parametrized index theorem for the algebraic $K$-theory Euler class. Acta Math., 190(1):1-104, 2003.

[Fer99] Davide L. Ferrario. A fixed point index for equivariant maps. Topol. Methods Nonlinear Anal., 13(2):313-340, 1999.

[Fer03] Davide L. Ferrario. A Möbius inversion formula for generalized Lefschetz numbers. Osaka J. Math., 40(2):345-363, 2003.

[FH93] Alexander Fel'shtyn and Richard Hill. Dynamical zeta functions, Nielsen theory and Reidemeister torsion. In Nielsen theory and 
dynamical systems (South Hadley, MA, 1992), volume 152 of Contemp. Math., pages 43-68. Amer. Math. Soc., Providence, RI, 1993.

[FH94] Alexander Fel'shtyn and Richard Hill. The Reidemeister zeta function with applications to Nielsen theory and a connection with Reidemeister torsion. K-Theory, 8(4):367-393, 1994.

[GN93] Ross Geoghegan and Andrew Nicas. Lefschetz trace formulae, zeta functions and torsion in dynamics. In Nielsen theory and dynamical systems (South Hadley, MA, 1992), volume 152 of Contemp. Math., pages 141-157. Amer. Math. Soc., Providence, RI, 1993.

[GN94] Ross Geoghegan and Andrew Nicas. Parametrized LefschetzNielsen fixed point theory and Hochschild homology traces. Amer. J. Math., 116(2):397-446, 1994.

[Jez95] Jerzy Jezierski. A modification of the relative Nielsen number of H. Schirmer. Topology Appl., 62(1):45-63, 1995.

[Jia83] Bo Ju Jiang. Lectures on Nielsen Fixed Point Theory, volume 14 of Contemporary Mathematics. American Mathematical Society, Providence, R.I., 1983.

[Kar78] Max Karoubi. K-Theory. Springer-Verlag, Berlin, 1978.

[Lef26] Solomon Lefschetz. Intersections and transformations of complexes and manifolds. Trans. Amer. Math. Soc., 28(1):1-49, 1926.

[LL89] Erkki Laitinen and Wolfgang Lück. Equivariant Lefschetz classes. Osaka J. Math., 26(3):491-525, 1989.

[LR03] Wolfgang Lück and Jonathan Rosenberg. The equivariant Lefschetz fixed point theorem for proper cocompact $G$-manifolds. In High-dimensional Manifold Topology, pages 322-361. World Sci. Publishing, River Edge, NJ, 2003.

[Lüc88] Wolfgang Lück. The equivariant degree. In Algebraic topology and transformation groups (Göttingen, 198\%), volume 1361 of Lecture Notes in Math., pages 123-166. Springer, Berlin, 1988.

[Lüc89] Wolfgang Lück. Transformation Groups and Algebraic KTheory, volume 1408 of Lecture Notes in Mathematics. SpringerVerlag, Berlin, 1989.

[Lüc99] Wolfgang Lück. The universal functorial Lefschetz invariant. Fund. Math., 161(1-2):167-215, 1999. 
[Mac71] Saunders MacLane. Categories for the Working Mathematician. Springer-Verlag, New York, 1971.

[Mil71] John Milnor. Introduction to Algebraic K-Theory. Princeton University Press, Princeton, N.J., 1971.

[Qui73] Daniel Quillen. Higher algebraic $K$-theory. I. In Algebraic $K$ theory, I: Higher K-theories (Proc. Conf., Battelle Memorial Inst., Seattle, Wash., 1972), pages 85-147. Lecture Notes in Math., Vol. 341. Springer, Berlin, 1973.

[Rei38] Karl Reidemeister. Automorphismen von Homotopiekettenringen. Math. Ann., 112:586-593, 1938.

[Ros94] Jonathan Rosenberg. Algebraic K-Theory and its Applications, volume 147 of Graduate Texts in Mathematics. Springer-Verlag, New York, 1994.

[Sch86] Helga Schirmer. A relative Nielsen number. Pacific J. Math., 122(2):459-473, 1986.

[Swa95] Richard G. Swan. Higher algebraic $K$-theory. In $K$-theory and algebraic geometry: connections with quadratic forms and division algebras (Santa Barbara, CA, 1992), volume 58 of Proc. Sympos. Pure Math., pages 247-293. Amer. Math. Soc., Providence, RI, 1995.

[tD79] Tammo tom Dieck. Transformation Groups and Representation Theory, volume 766 of Lecture Notes in Mathematics. Springer, Berlin, 1979.

[tD87] Tammo tom Dieck. Transformation Groups, volume 8 of de Gruyter Studies in Mathematics. Walter de Gruyter \& Co., Berlin, 1987.

[Wal85] Friedhelm Waldhausen. Algebraic $K$-theory of spaces. In Algebraic and geometric topology (New Brunswick, N.J., 1983), volume 1126 of Lecture Notes in Math., pages 318-419. Springer, Berlin, 1985.

[Wec41] Franz Wecken. Fixpunktklassen. II. Homotopieinvarianten der Fixpunkttheorie. Math. Ann., 118:216-234, 1941.

[Whi78] George W. Whitehead. Elements of Homotopy Theory, volume 61 of Graduate Texts in Mathematics. Springer-Verlag, New York, 1978.

[Wil84] Dariusz Wilczyński. Fixed point free equivariant homotopy classes. Fund. Math., 123(1):47-60, 1984. 
[Won91a] Peter Wong. Equivariant Nielsen fixed point theory for $G$-maps. Pacific J. Math., 150(1):179-200, 1991.

[Won91b] Peter Wong. On the location of fixed points of $G$-deformations. Topology Appl., 39(2):159-165, 1991.

[Won93] Peter Wong. Equivariant Nielsen numbers. Pacific J. Math., 159(1):153-175, 1993. 


\section{Index}

abelian category, 7

additive category, 7

additive invariant, 18

canpr, 69

canresiso, 90, 93

discrete group extension, 109

EI-category, 3

Endo(EI-Cat), 14

Endo(EI-Cat) ${ }_{\text {ord }}, 71$

equivariant Lefschetz class, 125

equivariant Nielsen class, 143

equivariant Nielsen invariants, 143

exact category, 8

extension functor, 6,68

ffRAut $(y)$-Circles, 88

ff $R \Gamma[y]$-Circles, 88

functorial equivariant Lefschetz invariant, 35

fundamental category, 3

$G$-CW-complex, 2

$G$-Jiang group, 148

$G$-Jiang space, 148

generalized equivariant Lefschetz invariant, 122

generalized local equivariant Lefschetz class, 130

$K_{0}$ of an exact category, 8

$\Lambda_{G}(X, f), 108,119$

Lefschetz fixed point theorem, 2

Lefschetz number, 1

Nielsen number, 142

order-respecting endofunctor, 66

$\phi$-end $\mathrm{d}_{\mathrm{ff} R \Gamma}, 9$

$\phi$-endomorphism, 9

$R \Gamma$-module, 5

free, 6

induction, 5

restriction, 5

refined equivariant Lefschetz fixed point theorem, 137

refined equivariant Lefschetz number, 113

refined incidence number, 115

retractive $G$-space, 48

splitting functor, 6,70

splitting functor Split $K, 72$

splitting morphism, 73

splitting results

basic, 74

combined, 83

finer, 81

geometric, 98

standard gap hypotheses, 145

trace map, 109, 113

generalized, 119, 120

$U(R, \Gamma, \phi), 11$

$U_{G}^{R}(X, f), 28$

universal additive invariant, 19

universal functorial equivariant Lefschetz invariant, 47

$\mathbb{Z} \pi_{1}\left(X^{H}(x), x\right)_{\phi_{x, w_{x}}^{\prime}}, 104$ 



\section{Curriculum Vitae}

\section{Julia Weber}

Geburtsdatum und -ort: 27.08.1977, Berlin

Familienstand:

ledig

Eltern:

Dr. Hans-Joachim Weber und Brigitte VenzkeWeber, geb. Venzke

Schulbildung

$$
\begin{array}{ll}
08 / 1983-01 / 1984 & \text { Nord-Grundschule Berlin } \\
02 / 1984-07 / 1987 & \text { Grundschule Dornholzhausen } \\
08 / 1987-06 / 1996 & \text { Gymnasium } \quad \text { Kaiserin-Friedrich- }
\end{array}
$$

Schule, Bad Homburg

03/1988 - 12/1988, John Strange Elementary School und

03/1989 - 12/1989 Eastwood Middle School, Indianapolis, Indiana

08/1993 - 06/1994 Ellsworth High School, Ellsworth, Maine (High School Diploma)

Hochschulreife 18.06.1996 Gymnasium Kaiserin-FriedrichSchule, Bad Homburg

Studium 10/1996-02/2002 Mathematik (Diplom), Nebenfach Volkswirtschaftslehre, RuprechtKarls-Universität Heidelberg

Prüfungen 07/1998 Licence de mathématiques und Maîtrise de mathématiques, École Normale Supérieure, Paris

05.02.2002 Diplom in Mathematik, Nebenfach Volkswirtschaftslehre, RuprechtKarls-Universität Heidelberg

Tätigkeiten 04/1998-09/1998, studentische Hilfskraft, Ruprecht10/1999 - 09/2000, Karls-Universität Heidelberg

$04 / 2001-09 / 2001$

10/2003 - 03/2004, wissenschaftliche Hilfskraft, Westfäli10/2004 - 03/2005 sche Wilhelms-Universität Münster

Praktika $\quad 02 / 1998-03 / 1998 \quad$ Springer-Verlag, Heidelberg 08/1999 - 10/1999 Deutsche Bundesbank, Frankfurt

Beginn der 05/2002

Westfälische Wilhelms-Universität Dissertation Münster, Mathematisches Institut, Betreuer: Prof. Dr. W. Lück 